\title{
A NEURAL NETWORK RECEIVER FOR EM-MWD COMMUNICATION
}

\author{
A Thesis \\ presented to \\ the Faculty of California Polytechnic State University, \\ San Luis Obispo
}

\author{
In Partial Fulfillment \\ of the Requirements for the Degree \\ Master of Science in Electrical Engineering
}

by

Timothy P. Whitacre

June 2011 
(C) 2011

Timothy Paul Whitacre

ALL RIGHTS RESERVED 


\section{Committee Membership}

Title: $\quad$ A Neural Network Receiver for EM-MWD Communication

Author: $\quad$ Timothy P. Whitacre

Date Submitted: June 2011

Committee Chair: Xiao-Hua (Helen) Yu

Committee Member: Samuel Agbo

Committee Member: Bryan Mealy 


\begin{abstract}
A Neural Network Receiver for EM-MWD Communication

Timothy P. Whitacre
\end{abstract}

Baseband digital communication in electro-magnetic measurement while drilling (EM-MWD) systems is often corrupted by non-white surface noise. The inability to reliably decode the transmitted signals in a noisy environment limits the depth at which EM-MWD systems can operate. Correlation receivers, which are optimal in the presence of additive white Gaussian noise, can be sub-optimal in the presence of various types of field noise at different drilling sites.

This thesis investigates the application of artificial neural networks (ANN) as communication receivers in EM-MWD baseband digital communication systems. The performances of various ANN architectures and training algorithms are studied and compared with conventional correlation receivers via computer simulations. Standard symbol error rate (SER) test results show that the NN receiver is able to adapt to site-specific noise and thus outperforms the traditional correlation receiver. 


\section{Acknowledgements}

First and foremost I want to acknowledge and thank my amazing wife. You have been my biggest support on this long journey to finish my degree. There were far too many nights stolen from you by this endeavor and I am excited to have the freedom to spend more time with you and our family. I next want to thank my advisor, Helen Yu, who has graciously helped me over many years with her time and advice. I am grateful for my employer, Applied Technologies, who hired a young man who didn’t know he had so many years ahead of him before finishing his degree. I also would like to thank my thesis committee members, who have taken time out of there busy schedules for my thesis defense. Last and definitely not least, I want to thank God for blessing me more than I could ever imagine. 


\section{Table of Contents}

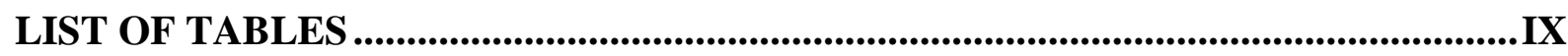

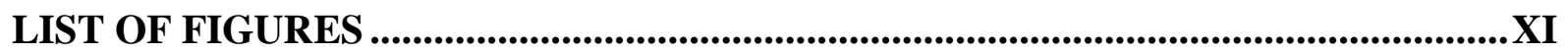

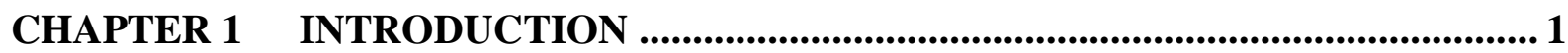

CHAPTER 2 BACKGROUND .............................................................................................. 4

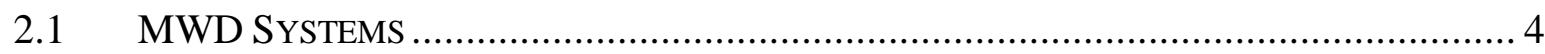

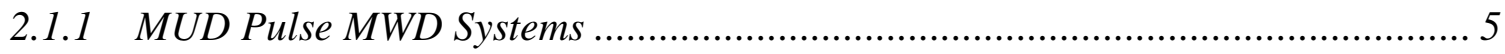

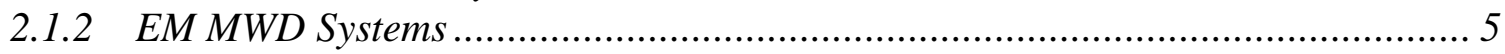

2.1.3 EM Advantages and Limitations .................................................................. 7

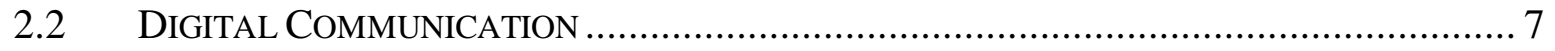

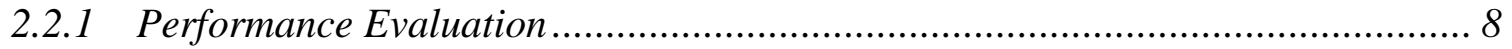

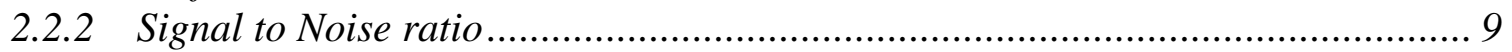

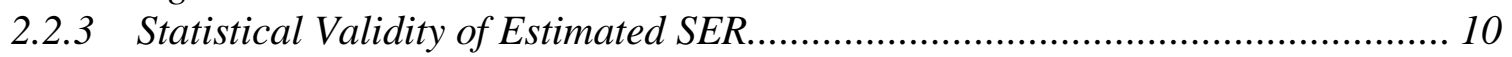

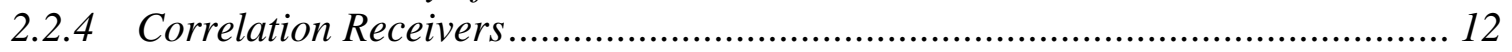

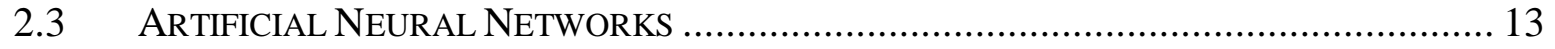

2.3.1 Biological Neural Network Fundamentals .................................................. 13

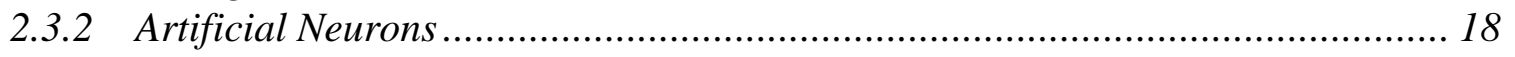

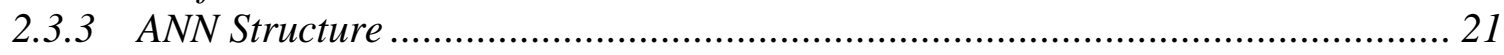

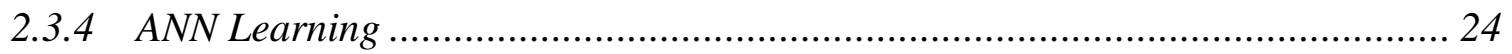

2.3.5 ANN Applications and Limitations................................................................ 27

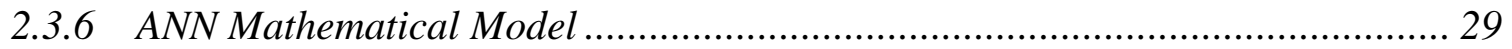

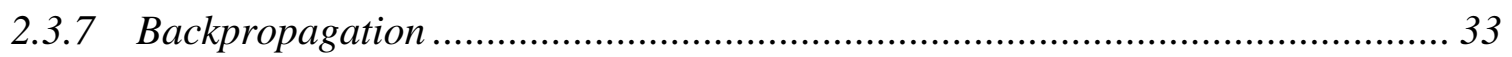

2.3.8 Gradient Calculation for Recurrent Networks................................................ 36

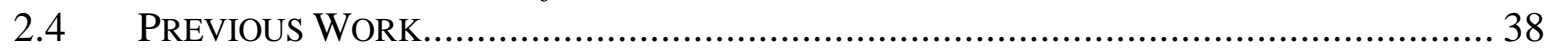

\section{CHAPTER 3 ARTIFICIAL NEURAL NETWORK RECEIVER ................................ 41}

3.1 DigITAL COMMUNICATION SYSTEM ...................................................................... 41

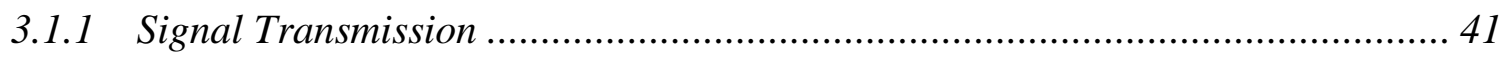

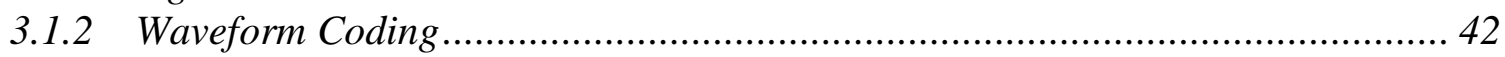

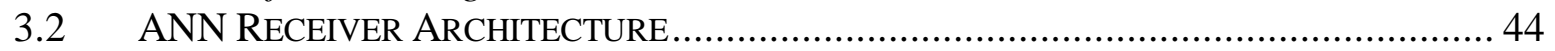

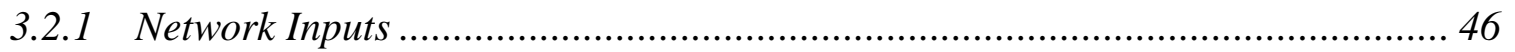

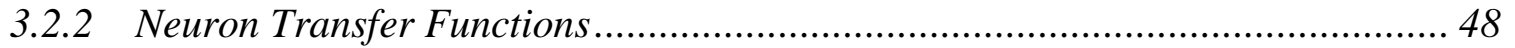

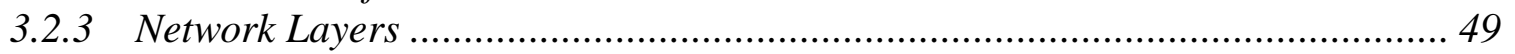

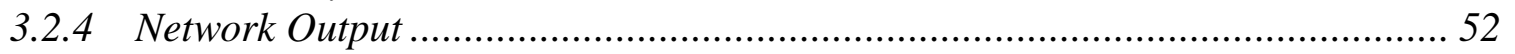

3.2.5 Similarities with Correlation Receivers........................................................... 53

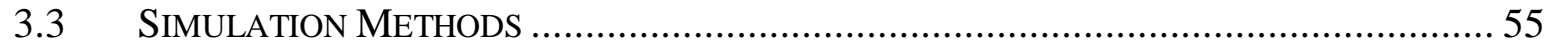

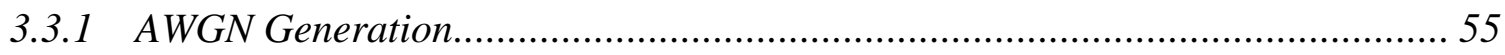

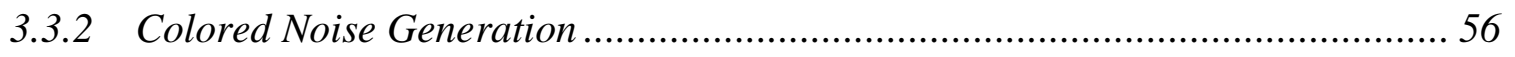

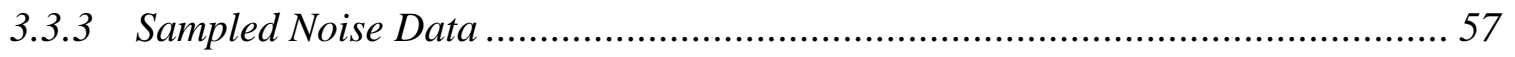


3.3.4 EM MWD Digital Communication Simulation …………............................... 58

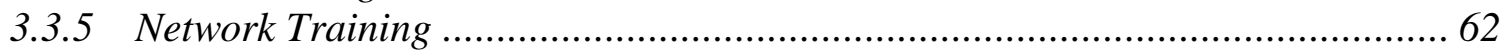

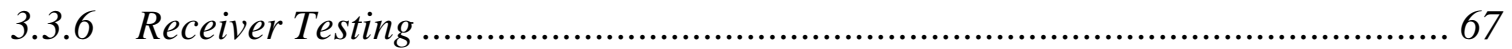

3.3.7 Correlation Receiver Implementation …………............................................. 69

CHAPTER 4 SIMULATIONS AND RESULTS …............................................................ 70

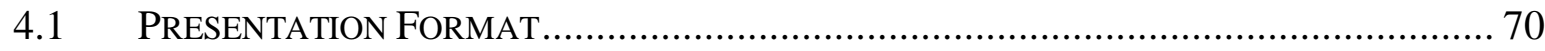

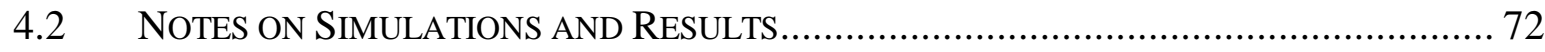

4.3 DEFAULT PARAMETERS FOR TRAINING FUNCTIONS .............................................. 74

4.4 SimULATIONS USING AWGN AND 4 SYMBOLS..................................................... 76

4.4.1 Single Layer Linear Network Simulations ………......................................... 76

4.4.2 Single Layer Linear Recurrent Network Simulations...................................... 84

4.4.3 Single Layer Non-Linear Network Simulations ………………........................ 88

4.4.4 Multi-Layer Non-Linear Network Simulations ……….................................... 91

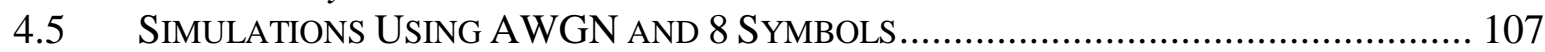

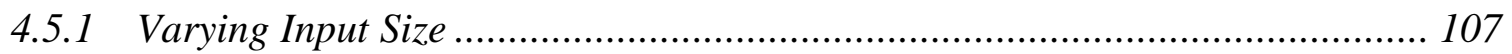

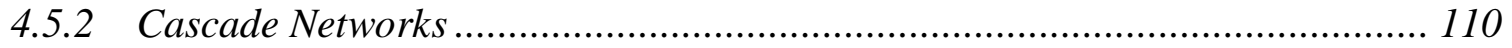

4.6 SimUlations USING Non-AWGN AND 4 SYMBOLS ............................................ 112

4.6.1 Varying Training Algorithm................................................................ 116

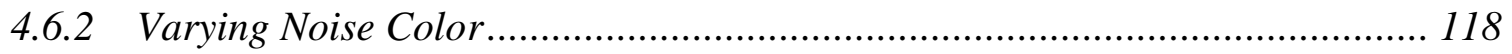

4.6.3 Frequency Domain Generated Shaped Noise ……....................................... 122

4.7 SimUlations USING Non-AWGN AND 8 SYMBOLS ........................................... 126

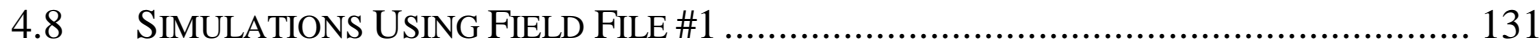

4.8.1 Training and Testing With The Same Noise Data....................................... 132

4.8.2 Training and Testing With Separate Noise Data ........................................... 135

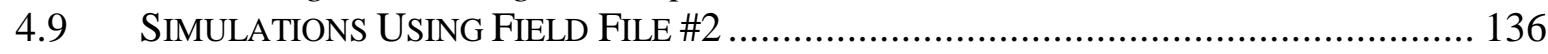

4.9.1 Training and Testing With The Same Noise Data......................................... 137

4.9.2 Training and Testing With Separate Noise Data .......................................... 138

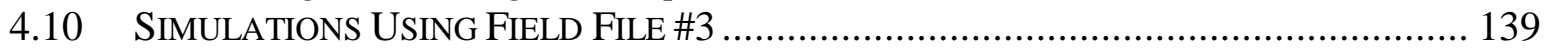

4.10.1 Training and Testing With The Same Noise Data..................................... 140

4.10.2 Training and Testing With Separate Noise Data ........................................ 141

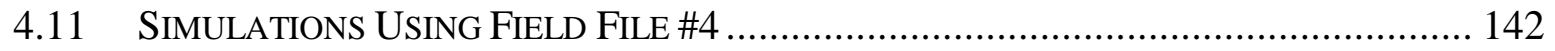

4.11.1 Training and Testing With The Same Noise Data..................................... 143

4.11.2 Training and Testing With Separate Noise Data ....................................... 144

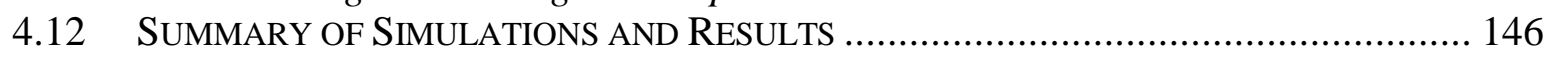

CHAPTER 5 CONCLUSIONS AND FUTURE WORK ............................................... 148

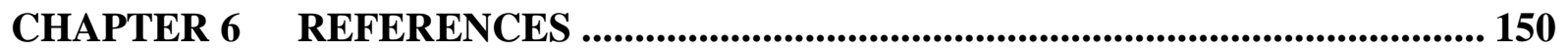

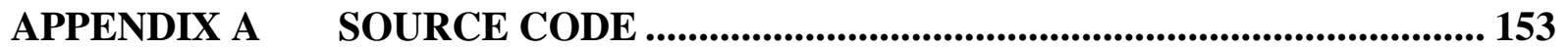

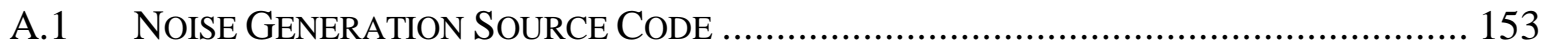

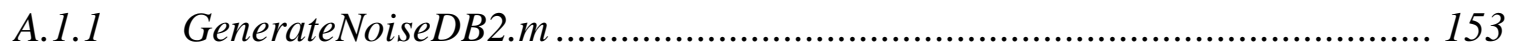

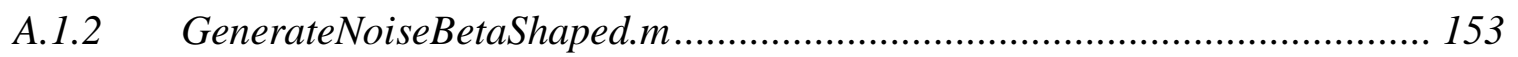

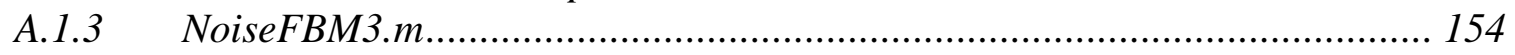


A.1.4 getNoiseFromFile3.m ................................................................... 155

A.2 CORRELATION RECEIVER SOURCE CODE........................................................ 157

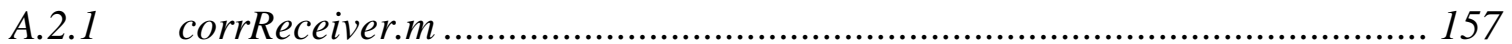

A.3 SuPPORT FunCTIONS SOURCE CODE.......................................................... 158

A.3.1 GenerateOrthogonalPatterns.m .................................................. 158

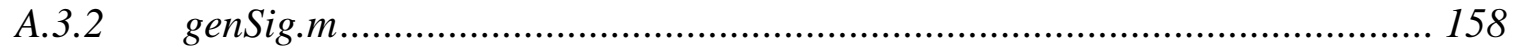

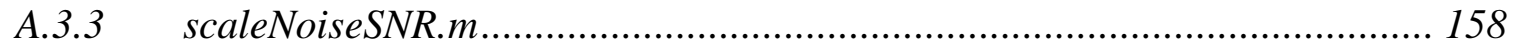

A.3.4 tapSampledSignal.m .................................................................... 158

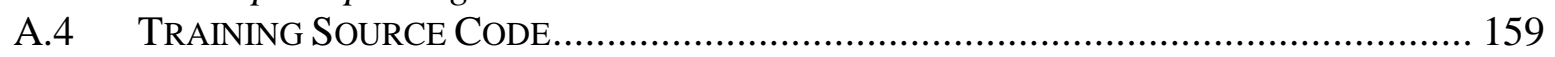

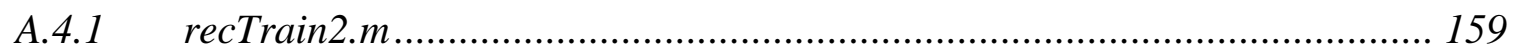

A.5 TeST SOURCE CODE ............................................................................. 161

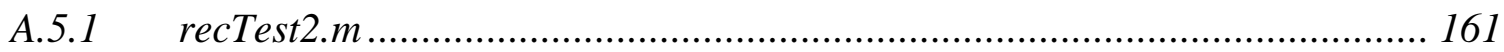

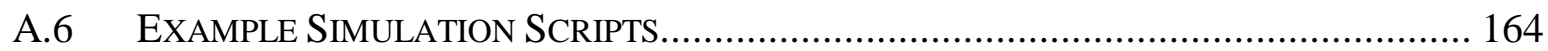

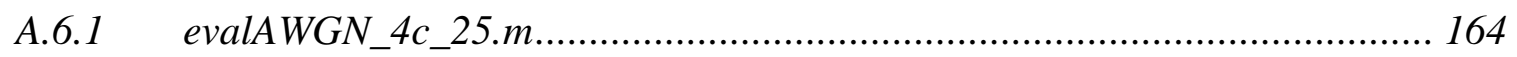

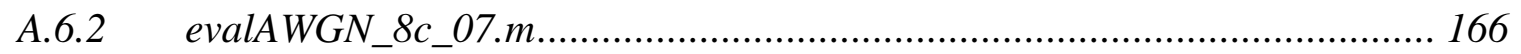

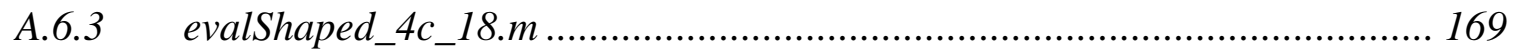

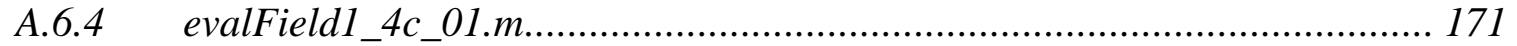

A.7 Data Plotting Source Code................................................................. 175

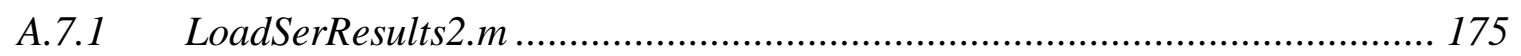

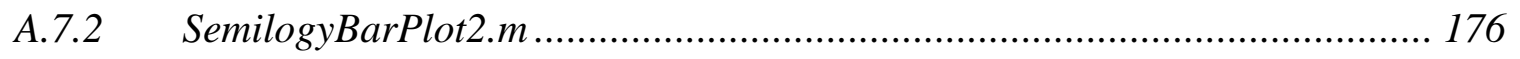




\section{List of Tables}

Table 2-1 Biological Neural Networks vs. Computers .................................................... 14

Table 3-1 Transfer Function Names ............................................................................ 49

Table 4-1 Example SER Data Table .............................................................................. 71

Table 4-2 Default Parameters for Training Function GDX .............................................. 74

Table 4-3 Default Parameters for Training Function LM.............................................. 76

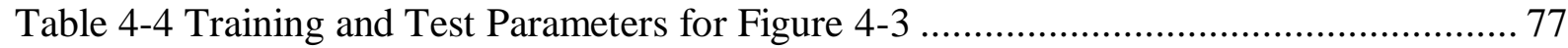

Table 4-5 SER Data for Figure 4-3 .......................................................................... 78

Table 4-6 SER Data for Figure 4-8 ...................................................................... 84

Table 4-7 Training and Test Parameters for Figure 4-10 .......................................... 85

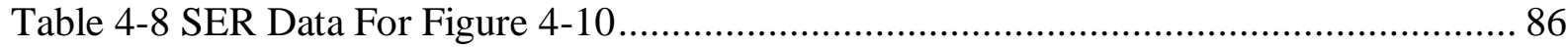

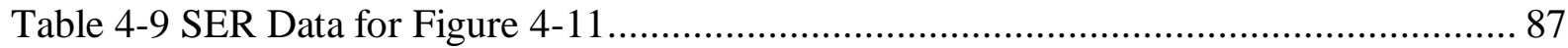

Table 4-10 Training and Test Parameters for Figure 4-15 ....................................... 88

Table 4-11 SER Data for Figure 4-13................................................................ 89

Table 4-12 Training and Test Parameters for Figure 4-15........................................... 90

Table 4-13 SER Data For Figure 4-15 .................................................................. 91

Table 4-14 Training and Test Parameters for Figure 4-17 ............................................ 91

Table 4-15 SER Data for Figure 4-17.................................................................... 92

Table 4-16 Training and Test Parameters for Figure 4-19........................................... 93

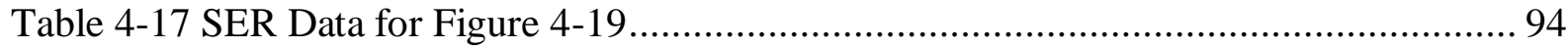

Table 4-18 Training and Test Parameters for Figure 4-21 ........................................... 95

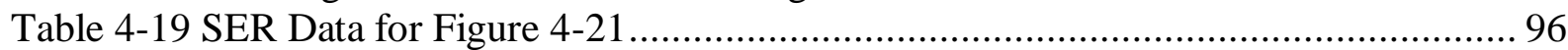

Table 4-20 Training and Test Parameters for Figure 4-23 ............................................. 97

Table 4-21 SER Data for Figure 4-23 .............................................................. 98

Table 4-22 Training and Test Parameters for Figure 4-25......................................... 99

Table 4-23 SER Data for Figure 4-25 .................................................................... 100

Table 4-24 SER Data for Figure 4-26.................................................................... 101

Table 4-25 Training and Test Parameters for Figure 4-28, Figure 4-29, Figure 4-33,

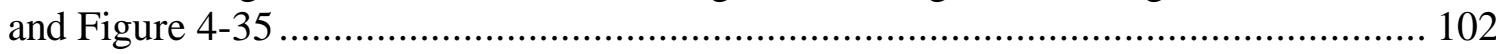

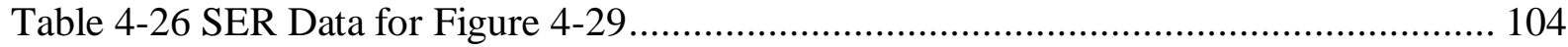

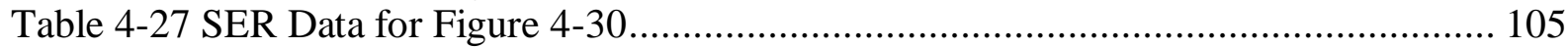

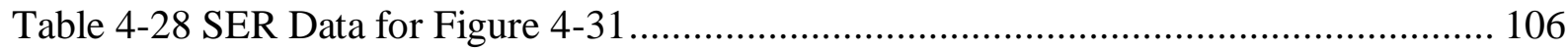

Table 4-29 Training and test parameters for Figure 4-33 f....................................... 107

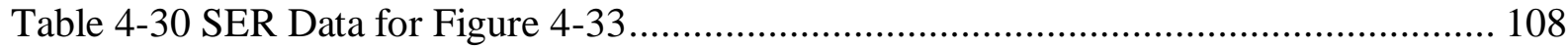

Table 4-31 Training and test parameters for Figure 4-35 f....................................... 109

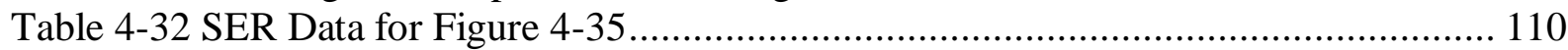

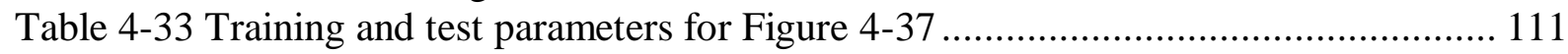

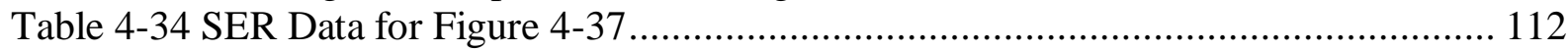

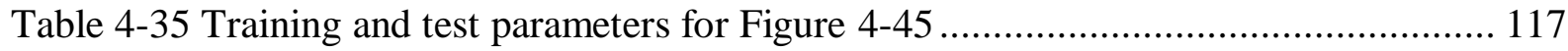

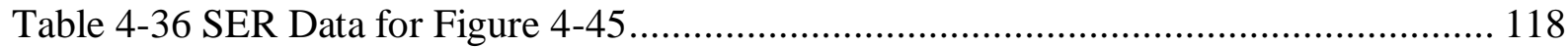

Table 4-37 Training and test parameters for Figure 4-47, Figure 4-48, and Figure 4-49..... 119

Table 4-38 SER Data for Figure 4-47...................................................................... 120

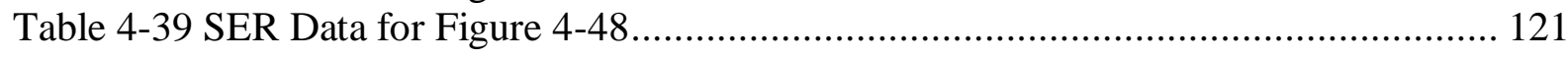

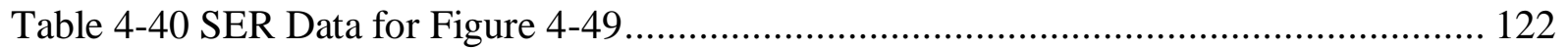


Table 4-41 Training and test parameters for Figure 4-51, Figure 4-52, and Figure 4-53..... 123

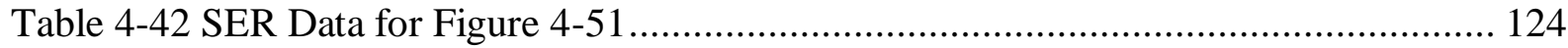

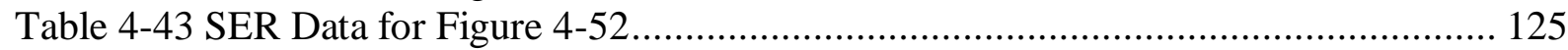

Table 4-44 SER Data for Figure 4-53 ............................................................ 125

Table 4-45 Training and test parameters for Figure 4-55, Figure 4-56, and Figure 4-57..... 127

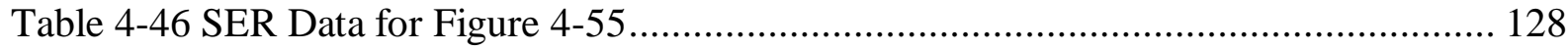

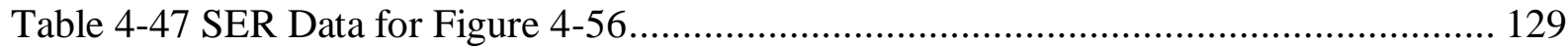

Table 4-48 SER Data for Figure 4-57 .................................................................. 130

Table 4-49 Training and test parameters for field file simulations. ................................. 133

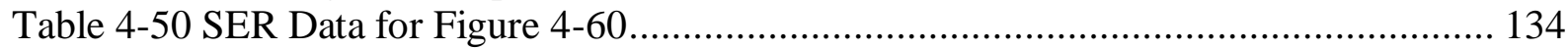

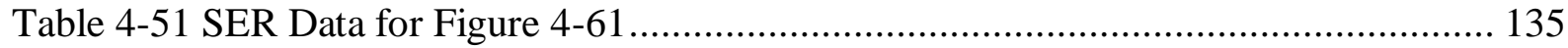

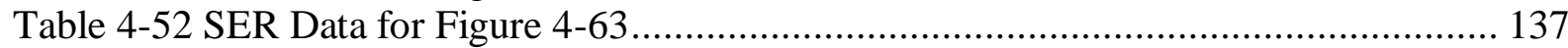

Table 4-53 SER Data for Figure 4-64 .................................................................. 138

Table 4-54 SER Data for Figure 4-66..................................................................... 140

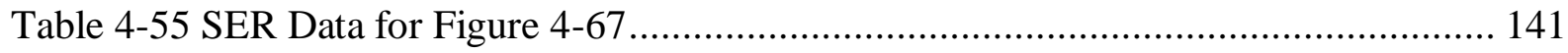

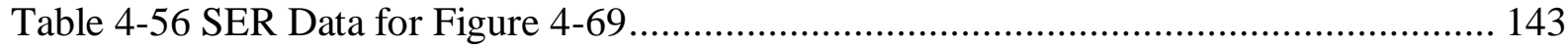

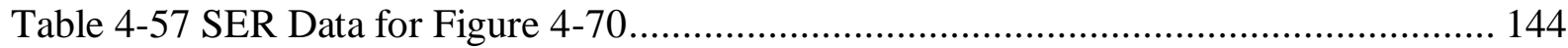




\section{List of Figures}

Figure 2-1 Basic Electromagnetic MWD System ........................................................ 6

Figure 2-2 EM MWD Digital Communication System ................................................. 8

Figure 2-3 Biological Neuron Components .................................................................. 15

Figure 2-4 Temporal Response of Biological Neuron............................................. 16

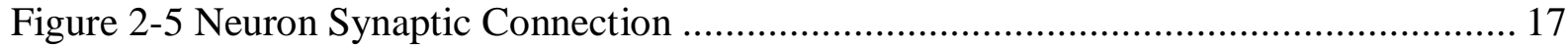

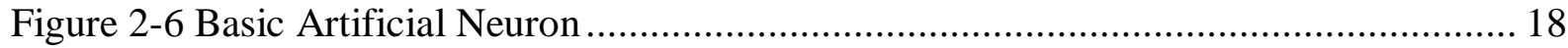

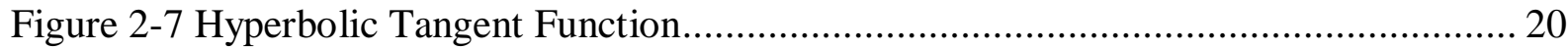

Figure 2-8 Simple Artificial Neural Network ............................................................ 23

Figure 2-9 Artificial Neural Network Layers.......................................................... 24

Figure 2-10 Characteristics of Different Learning Rates......................................... 26

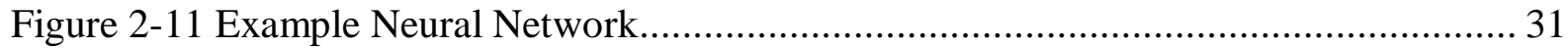

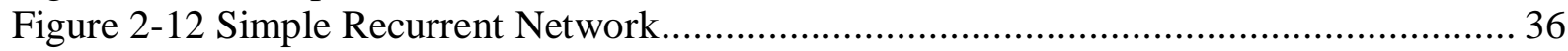

Figure 3-1 Flow Chart of a Simple Neural Network Receiver........................................... 44

Figure 3-2 4-bit symbol made up of 8 samples ............................................................ 46

Figure 3-3 Input formatting for a 4-input network used to process the symbol of Figure

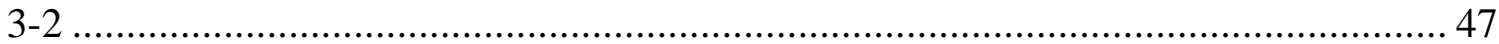

Figure 3-4 Neuron Transfer Functions ................................................................... 48

Figure 3-5 Types of Network Layer Interconnections................................................ 50

Figure 3-6 Example Neuron Connections for Feed Forward Networks ............................. 51

Figure 3-7 Example Neuron Connections for Recurrent Networks ................................. 51

Figure 3-8 Example Neuron Connections for Cascade Networks....................................... 52

Figure 3-9 Neural Network Inputs and Outputs .......................................................... 53

Figure 3-10 Bipolar 4-Ary Orthogonal Symbol Set Sampled at 200Hz with 50ms pulse

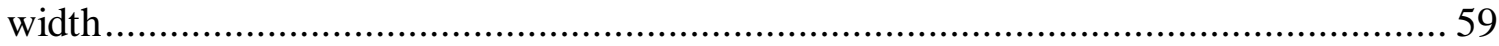

Figure 3-11 Transmission Signal consisting of 2 random permutations of the symbol

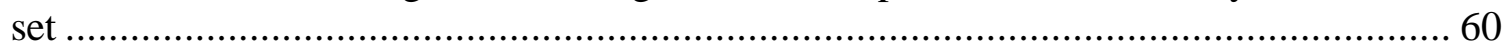

Figure 3-12 EM MWD Simulation Signal Stages .................................................... 61

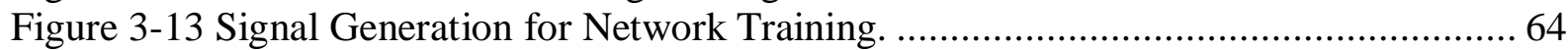

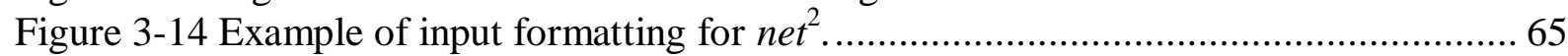

Figure 3-15 Training targets for a symbol set containing 4 symbols .............................. 66

Figure 3-16 Neural Network Receiver Training Flow Chart ....................................... 67

Figure 3-17 EM Digital Communication Receiver Performance Evaluation Flow Chart ....... 68

Figure 4-1 Example SER Plot. .............................................................................. 71

Figure 4-2 Layer Connections for Neural Networks of Figure 4-3 .................................. 76

Figure 4-3 Single Layer Linear Network Simulation Results for AWGN with 4

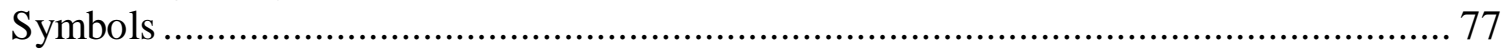

Figure 4-4 Input Weights Compared with Correlation Receiver Reference Signals.............. 79

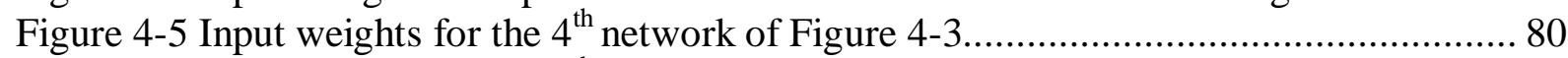

Figure 4-6 Input weights for the $7^{\text {th }}$ network of Figure 4-3 ........................................ 81

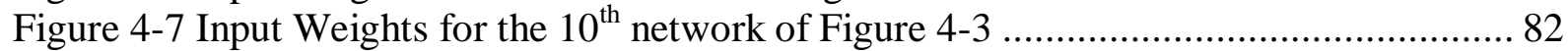

Figure 4-8 Single Layer Linear Network Simulation Results with Weighted Post

Processing For AWGN with 4 Symbols.............................................................. 83

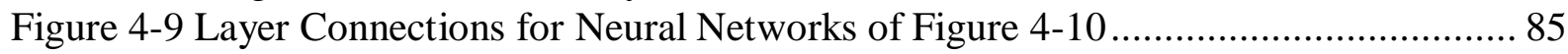


Figure 4-10 Single Layer Linear Recurrent Network Simulation Results for AWGN

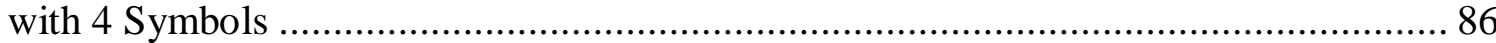

Figure 4-11 Single Layer Linear Recurrent Network Simulation Results with

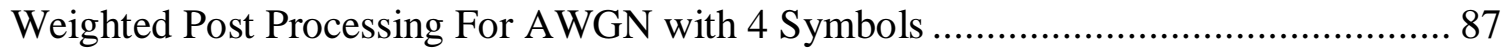

Figure 4-12 Layer Connections for Neural Networks of Figure 4-15 ............................ 88

Figure 4-13 Single Layer SIG Network Simulation Results for AWGN with 4

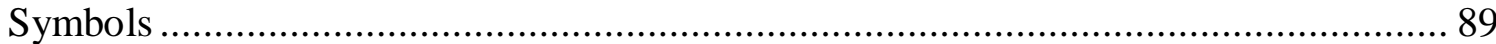

Figure 4-14 Layer Connections for Neural Networks of Figure 4-15 .............................. 89

Figure 4-15 Single Layer RB Network Simulation Results for AWGN with 4 Symbols ....... 90

Figure 4-16 Layer Connections for Neural Networks of Figure 4-17 .............................. 91

Figure 4-17 Varying Input Size of 2 layer SIG Networks for AWGN with 4 Symbols ......... 92

Figure 4-18 Layer Connections for Neural Networks of Figure 4-19 ............................. 93

Figure 4-19 Varying Input Layer Size of 2 Layer SIG Networks for AWGN with 4

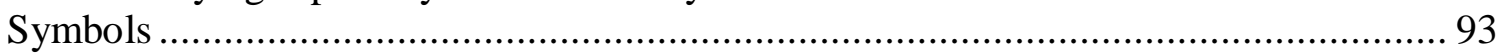

Figure 4-20 Layer Connections for Neural Networks of Figure 4-21 ............................. 95

Figure 4-21 Varying Size of Cascaded Nonlinear Networks with Linear Output Layer

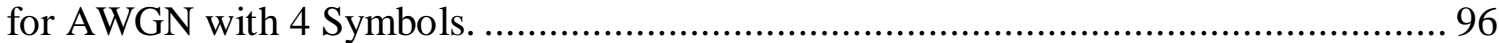

Figure 4-22 Layer Connections for Neural Networks of Figure 4-23 ............................... 97

Figure 4-23 Pseudo Cascade Correlation Networks for AWGN with 4 Symbols ................. 98

Figure 4-24 Layer Connections for Neural Networks of Figure 4-25 and Figure 4-26 ......... 99

Figure 4-25 Varying the training algorithm of a single linear layer for AWGN with 4 symbols.

Figure 4-26 Same as Figure 4-25 with Reduced Training for the Levenberg-Marquardt

Algorithm. The number of epochs per batch was reduced to 20 from 100 ................. 101

Figure 4-27 Layer connections for neural networks of Figure 4-28, Figure 4-29, Figure

4-30 and Figure 4-35. a) Connections for 2 layer cascade networks. b)

Connections for 2 layer networks. c) Connections for single layer network. 102

Figure 4-28 No Post-Noise Filtering for AWGN with 4 symbols................................... 103

Figure 4-29 30Hz $2^{\text {nd }}$ Order Low Pass Post-Noise Filtering for AWGN with 4 symbols .... 104

Figure 4-30 $20 \mathrm{~Hz}$ 2nd Order Low Pass Post-Noise Filtering for AWGN with 4

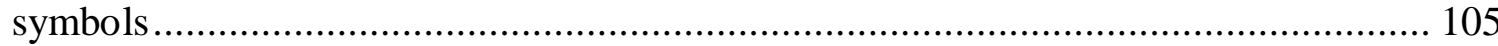

Figure 4-31 $10 \mathrm{~Hz}$ 2nd Order Low Pass Post-Noise Filtering for AWGN with 4

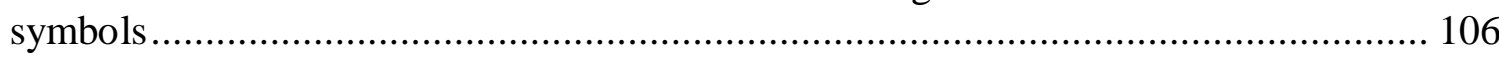

Figure 4-32 Layer connections for neural networks of Figure 4-33 ............................... 107

Figure 4-33 Varying input size of a single layer linear network for AWGN with 8

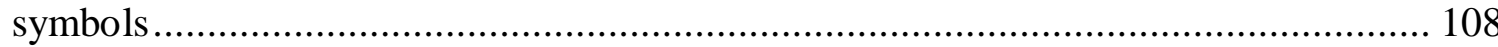

Figure 4-34 Layer connections for neural networks of Figure 4-35............................... 109

Figure 4-35 Varying Input Size of a 2-Layer Non-Linear Network for AWGN with 8

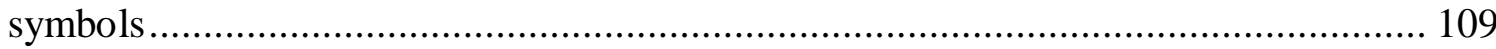

Figure 4-36 Layer connections for neural networks of Figure 4-37 ............................ 110

Figure 4-37 Cascade Networks for AWGN with 8 symbols ....................................... 111

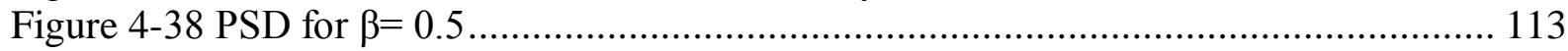

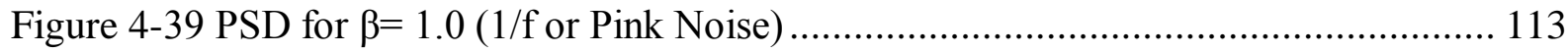

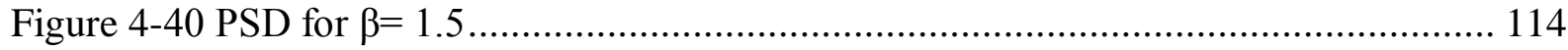

Figure 4-41 PSD For $\beta=0.5$ Centered at $10 \mathrm{~Hz}$ With a $4 \mathrm{~Hz}$ Plateau Width...................... 114

Figure 4-42 PSD For $\beta=1.0$ Centered at $10 \mathrm{~Hz}$ With a $4 \mathrm{~Hz}$ Plateau Width...................... 115 
Figure 4-43 PSD For $\beta=1.5$ Centered at $10 \mathrm{~Hz}$ With a $4 \mathrm{~Hz}$ Plateau Width...................... 115

Figure 4-44 Layer connections for neural networks of Figure 4-45 ............................. 116

Figure 4-45 Varying Training Algorithm for Single Layer Linear and Non-Linear

Networks with Pink Noise

Figure 4-46 Layer connections for neural networks of Figure 4-47, Figure 4-48, and

Figure 4-49

Figure 4-47 Networks Tested With Shaped Noise $($ Beta $=0.5)$ and 4 Symbols

Figure 4-48 Networks Tested With Shaped Noise (Beta $=1.0)$ and 4 Symbols

Figure 4-49 Networks Tested With Shaped Noise $($ Beta $=1.5)$ and 4 Symbols

Figure 4-50 Layer connections for neural networks of Figure 4-51, Figure 4-52, and

Figure 4-53

Figure 4-51 Networks Tested with Shaped Noise of Figure 4-41 (Beta $=0.5$, Center $=$

$10 \mathrm{~Hz}$, Width $=4 \mathrm{~Hz}$ )

Figure 4-52 Networks Tested with Shaped Noise of Figure 4-42 (Beta = 1.0, Center $=$

$10 \mathrm{~Hz}$, Width $=4 \mathrm{~Hz}$ )

Figure 4-53 Networks Tested with Shaped Noise of Figure 4-43 $($ Beta $=1.5$, Center $=$

$10 \mathrm{~Hz}$, Width $=4 \mathrm{~Hz}$ )

Figure 4-54 Layer connections for neural networks of Figure 4-55, Figure 4-56, and

Figure 4-57

Figure 4-55 Networks Tested With Shaped Noise $($ Beta $=0.5)$ and 8 Symbols

Figure 4-56 Networks Tested With Shaped Noise $($ Beta $=1.0)$ and 8 Symbols 128

Figure 4-57 Networks Tested With Shaped Noise $($ Beta $=1.5)$ and 8 Symbols 129

Figure 4-58 Frequency Content of Field File \#1 131

Figure 4-59 Layer connections for neural networks used with field file simulations .......... 132

Figure 4-60 Same Training and Test Noise With Field File \#1 and 4 Symbols ................. 133

Figure 4-61 Separate Training and Test Noise with Field File \#1 and 4 Symbols .............. 135

Figure 4-62 Frequency Content of Field File \# ........................................................ 136

Figure 4-63 Same Training and Test Noise With Field File \#2 and 4 Symbols .................. 137

Figure 4-64 Separate Training and Test Noise with Field File \#2 and 4 Symbols .............. 138

Figure 4-65 Frequency Content of Field File \#3 ...................................................... 139

Figure 4-66 Same Training and Test Noise with Field File \#3 and 4 Symbols ................... 140

Figure 4-67 Separate Training and Test Noise with Field File \#3 and 4 Symbols ............... 141

Figure 4-68 Frequency Content of Field File \#4 ....................................................... 142

Figure 4-69 Same Training and Test Noise with Field File \#4 and 4 Symbols .................. 143

Figure 4-70 Separate Training and Test Noise with Field File \#4 and 4 Symbols .............. 144

Figure 4-71 - Mean squared error performance during training of 3-layer cascade network

Figure 4-72 - Mean squared error performance during training of 3-layer feed forward network 


\section{Chapter 1 Introduction}

Modern directional drilling has increased the precision of drilling wells for oil, gas, and geothermal uses. It utilizes various sensors located near the drill bit as a means of transferring readings from sensors to drilling operators. Accelerometers, magnetometers, and gyros are used to determine the location and attitude of the drill bit with respect to gravity, magnetic north, and true north respectively. This information is necessary to ensure wells are drilled as planned within lease lines as well as drilled to the desired target location along the prescribed well profile [1].

Transferring sensor measurements to the surface, while drilling, commonly known as measurement while drilling (MWD), enables the drilling operators to guide the drill bit with increased precision. The data from the sensors are transferred to the drilling operators at the surface in two primary ways [2].

The first transmission method uses pressure signals communicated through a column of fluid present in the well. This form of communication is known as mud pulsing. The pressure signals generated by the tool (sensors and associated electronics) are received by a pressure sensor near the surface of the well. The pressure signals are then decoded for the drilling operator. The main sources of noise associated with mud pulsing MWD are from pumps at the surface and drill motors beneath the surface [1].

The second method of communicating sensor readings is through electromagnetic waves (EM). Either a voltage is modulated across a gap, or a current is induced in the drill string by the EM MWD tool. The resulting EM waves propagate through the earth and are

detected by equipment at the well surface. The majority of noise that the EM MWD systems 
must deal with is generated at the well surface. Drill pumps, motors, and even lightning can generate noise, which degrades EM MWD system performance [3].

In order for the EM signal to be reliably detected, a receiver must be able to deal with the various noise sources near the drill rig. There are a number of methods used to aid in the filtering and decoding of EM signals in the presence of noise. At least one MWD company uses a correlation receiver preceded by various analog and digital filters [4]. This method proves to work well when the spectral content of the noise and the transmitted signals do not overlap significantly. The ability of the correlation receiver to correctly decode the transmitted symbols decreases as the noise spectrum encroaches on the signal spectrum, and as the signal to noise ratio decreases [5]. The majority of the power contained in the transmitted signal is in the low frequency band because the earth tends to act as a low-pass filter for EM waves. The noise sources on or near the drill rig usually have large power spectral densities in the same frequency band [4]. This explains why there can be much difficulty associated with the reception of EM signals under certain conditions.

This thesis investigates the application artificial neural networks as communication receivers to detect EM signals at the well surface. Artificial neural networks are the result of attempts to solve problems by mimicking the manner in which biological neural networks (i.e. brains) function. Biological neural networks are able to learn, adapt, and process data nonlinearly. They are able to extract information in the presence of considerable amounts of noise [6]. It is expected that the neural network receiver is able to decode signals in the presence of noise that would normally be difficult for a correlation receiver.

This thesis is organized as follows. Chapter Two provides a more detailed description of the problem statement. The operation of MWD systems is addressed with emphasis on the 
communication of sensor data. In addition, relevant background on communication theory will also be reviewed, followed by an overview of the fundamental concepts and purposes of neural networks. Chapter Two concludes with a review of previously published work in the areas of neural networks, digital receivers, and EM MWD communication. Chapter Three describes the architecture of neural network receivers. Chapter Four outlines the computer simulation results and analysis. Finally, conclusions and suggestions for future works are presented in chapter Five. 


\section{Chapter 2 Background}

\subsection{MWD Systems}

Monitoring While Drilling (MWD) systems allow for near real-time information on the orientation of the down-hole drill bit in oil and gas drilling applications. This information is necessary in order for the actual profile to match the desired profile of the well. Additionally, various sensors readings transmitted to the drilling operator can be used to optimize the location of the finished well for production of oil or gas. Many wells are often drilled from the same platform, increasing the probability of collisions with previously drilled wells. This coupled with the need to legally ensure the placement of wells within lease lines and other constraints makes the timely feedback of drill bit position and orientation extremely important.

There are difficulties associated with providing reliable transmission of the information from the down-hole tool to the surface in a timely manner. Noise from location to location can vary drastically, as well as earth formation, and the depth of drilling. In order for MWD companies to competitive they must be able to provide consistent reliable communication while drilling.

There are two widely used methods for the transmission of sensor readings from down-hole to the drilling operators at the surface. Mud pulsing is the older of the two and involves generating pressure waves in the drilling fluid to communicate from the tool to the surface. The second method creates electromagnetic waves that pass through the earth from the tool to the surface. This method is often referred to as EM. MWD companies continually strive to improve the performance of these transmission options [1]. 


\subsubsection{MUD Pulse MWD Systems}

Mud-pulse systems use a column of fluid, mud, to transmit data encoded pressure signals uni-directionally from the tool to the surface. Opening and closing a valve through which the mud passes create the pressure signals. The pressure pulses are converted to electrical signals by a pressure transducer located at the surface. The message contained in the series of pulses is then decoded and the information is presented to the drilling operator. Alternate uses of the mud include powering the drilling motors located near the down-hole drill bit, lubricating the mud motors, and removing the cuttings from the well.

Nearly all of the noise in a mud pulse system comes from pressure fluctuations in the fluid caused by sources other than the mud pulse tool. Noise introduced after the pressure signal is converted to an electronic signal is negligible. The attenuation of the pressure signal over distance is dependent upon the type of drilling fluid used, increasing as the density decreases. The data rates for mud-pulse systems are slow, usually in the 1 to 2 bits per second range.

\subsubsection{EM MWD Systems}

EM MWD systems communicate by either modulating a voltage source across an isolated gap, or inducing a current in the drill string. The resulting electromagnetic waves are sent to the surface where they are detected by an antenna. The data is then decoded and presented to the operator. An example of an EM MWD system is shown in Figure 2-1. 


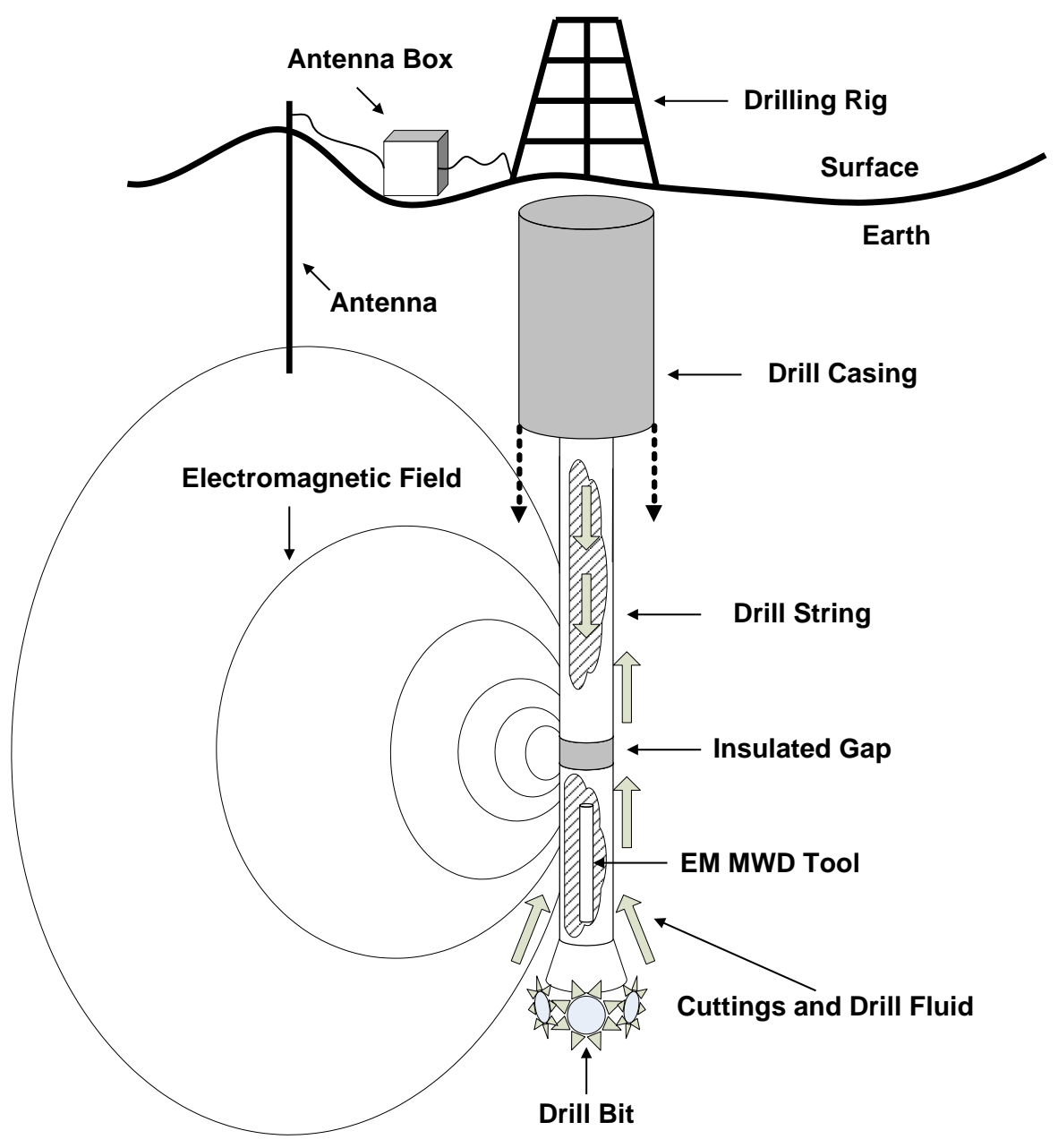

Figure 2-1 Basic Electromagnetic MWD System

Numerous potential noise sources exist when using electromagnetic wave telemetry.

Motors, power lines, lightning, and more can inject noise into the receiving antenna. The power spectral density of the noise often has spikes and is usually non-stationary. The strength of the EM signal degrades significantly as the distance between the MWD tool and antenna increases. 


\subsubsection{EM Advantages and Limitations}

EM systems provide higher data rates than mud-pulse systems. However, they are unable to reach the same depth as their mud-pulse counterparts due to the attenuation of the EM signal as it passes through the earth. EM systems are able to communicate in under balanced drilling situations where the drilling fluid density is too small for mud-pulse systems. The EM communication system does not use any moving parts, whereas the mudpulse systems must physically open and close valves. Noise abounds in both systems, but EM systems have a much lower signal-to-noise ratio (SNR) as the depth of the tool increases.

\subsection{Digital Communication}

Analog and digital communication systems transfer information from a transmitter, through a transmission channel, to a receiver. Both types of systems aim to provide the receiver with an exact replica of the information sent by the transmitter.

The information transferred with an analog communication system has infinite resolution. The goal of the receiver is to preserve the fidelity of the information.

With digital communication systems, information is first quantized into a sequence of digital symbols, also known as a bit stream. Thus the information sent is not infinite in resolution, but is represented by a finite set of digital symbols, each made up of $\boldsymbol{k}$ bits. These digital symbols are converted to digital waveforms that are compatible with the transmission channel through a process known as modulation. A transmitter is used to pass the digital waveform through the transmission channel to a receiver. The waveform is corrupted by various noise sources and the channel transfer function as it travels to the receiver. The 
receiver demodulates the received waveform into a bit stream, which ideally matches the bit stream of the transmitter [5].

The digital communication system addressed by this thesis is shown in Figure 2-2

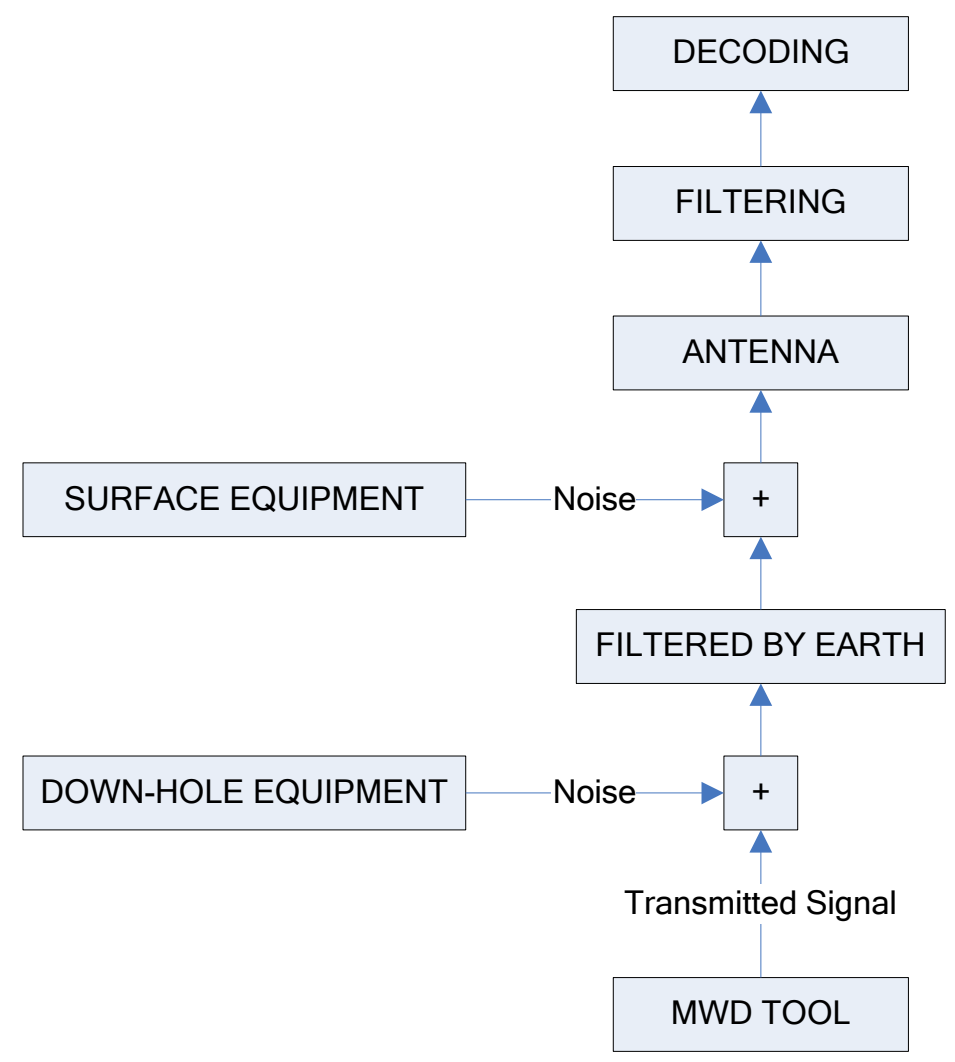

Figure 2-2 EM MWD Digital Communication System

\subsubsection{Performance Evaluation}

Digital communication systems are usually judged by the probability of incorrectly identifying a digital symbol from a transmitted waveform. The probability of bit error, otherwise known as bit error rate (BER), of a system is the specific way of normally evaluating this criterion. The BER for a communication system is often plotted against the signal to noise ratio, and results in a waterfall shape, with the probability of error decreasing 
as the SNR increases. The probability of bit error is a special case of the probability of symbol error (SER) where the length of the symbol is one bit. The neural network receivers in this thesis are trained to identify symbols containing more than one bit, thus the SER is used exclusively.

\subsubsection{Signal to Noise ratio}

When dealing with communication systems, the signal to noise ratio is usually defined as the energy per bit to noise spectral density ratio. Most communication systems endeavor to maximize this ratio, which in turn increases the probability of correctly detecting the signal, or in the analog case, preserve the transmitted waveform. The ratio is usually expressed in decibels and is shown in equation 2-1, where $E_{b}$ is the energy per bit, and $N_{0}$ is the noise spectral density.

$$
S N R_{1}=10 \cdot \log \left(\frac{E_{b}}{N_{0}}\right)
$$

The noise power spectral density is in units of Watts per Hertz and represents the amount of power contained at each frequency band in the spectrum. White noise has a constant power spectral density across all frequencies and is often used as the corrupting source when analyzing communication systems.

The noise power spectral density for non-white noise is not constant across all

frequencies. Equation 2-4 shows the SNR definition used in this thesis, where $E_{\text {sig }}$ is the 
signal energy, and $E_{\text {noise }}$ is the noise energy. $E_{\text {sig }}$ and $E_{\text {noise }}$ are defined in 2-2 and 2-3 respectively, where $N$ is the number of samples for each signal.

$$
\begin{gathered}
E_{\text {sig }}=\sum_{n=1}^{N} \operatorname{sig}(n)^{2} \\
E_{\text {noise }}=\sum_{n=1}^{N} n \text { oise }(n)^{2} \\
S N R_{2}=10 \cdot \log \left(\frac{E_{\text {sig }}}{E_{\text {noise }}}\right)
\end{gathered}
$$

\subsubsection{Statistical Validity of Estimated SER}

The SER obtained through simulations is a binomial proportion because each of the symbols transmitted results in either a success or failure. As the number of transmitted symbols increases, the percentage of symbols that were received in error approaches the actual probability of symbol error. In this thesis, the SER is an estimate of a given receiver's probability of decoding the wrong symbol. One way to judge the reliability of an experimentally derived estimate is with confidence intervals. A confidence interval is usually stated as a percentage, such as a $95 \%$ confidence interval. As it relates to this project, the percentage represents how likely that the true probability of symbol error for a given receiver lies within the calculated confidence interval from a particular experiment. For example, 95\% of identically run experiments will result in the true probability of symbol being within the calculated 95\% confidence interval for each experiment.

There exist a variety of methods for estimating confidence intervals for binomial proportions. [7] has shown that the Agresti-Coull confidence interval is more accurate than 
the standard Wald confidence interval, especially as the true probability approaches 0 or 1 . Equation 2-5 is the standard Wald confidence interval, with $n$ equal to the total number of samples, $\hat{p}$ equal to the number of errors, $x$, divided by $n$, and $z$ taken from the standard normal distribution for the desired confidence percentile.

$$
C I_{W A L D}=\hat{p} \pm z \sqrt{\frac{\hat{p}(1-\hat{p})}{n}}
$$

Equation 2-6 is the Agresti-Coull confidence interval.

$$
C I_{A C}=\frac{x+\frac{z^{2}}{2}}{n+z^{2}}+z \sqrt{\frac{\left(\frac{x+\frac{z^{2}}{2}}{n+z^{2}}\right)\left(1-\frac{x+\frac{z^{2}}{2}}{n+z^{2}}\right)}{n+z^{2}}}
$$

This equation is similar in form to the Wald interval, which can be seen by using the following variable substitutions.

$$
\begin{gathered}
\tilde{x}=x+\frac{z^{2}}{2} \\
\tilde{n}=n+z^{2} \\
\tilde{p}=\frac{\tilde{x}}{\tilde{n}}
\end{gathered}
$$

Using these substitutions with equation 2-6 results in the following equation, which is in the same form as the standard Wald interval. 


$$
C I=\tilde{p} \pm z \sqrt{\frac{\tilde{p}(1-\tilde{p})}{\tilde{n}}}
$$

The size of the confidence interval is thus given by equation 2-11.

$$
W_{C I}=2 z \sqrt{\frac{\tilde{p}(1-\tilde{p})}{\tilde{n}}}
$$

The confidence intervals obtained by applying equation 2-10 to the SER test results will be used to validate the comparisons made.

\subsubsection{Correlation Receivers}

The correlation receiver contains $M$ individual correlators, where $M$ is the number of symbols contained the code set. Each correlator integrates the product of a received signal, $r(t)$, with a replica of one of the symbol waveforms, $s_{i}(t)$ over a single symbol interval, $T$, as is shown in Equation 2-12.

$$
z_{i}(T)=\int_{0}^{T} r(\tau) s_{i}(\tau) \delta \tau, i=1, \ldots, M
$$

When the received signal is sampled, the discrete version of the correlation receiver is used.

$$
z_{i}(N)=\sum_{k=0}^{N} r(k) s_{i}(k), i=1, \ldots, M
$$

The decision as to which symbol was most likely transmitted is made by choosing the correlator with the largest output. This has been shown to be the best possible choice as long as each of the symbols has the same probability of being transmitted and the received signal is corrupted by AWGN [5]. 


\subsection{Artificial Neural Networks}

The field of artificial neural networks (ANN) aims to process data in a way similar to that of biological neural networks, i.e. brains. The brain is amazing; it handles thousands of nonlinear inputs simultaneously and learns to process them into something meaningful. It can easily pick out important inputs, and disregard others. Think about the very fact that you are reading this. You are able to process raw image data into intelligible letters, words, phrases, and thoughts. You are even able to extract meaning out of noisy data. For example, you porlbably arje wndorenig why I am msislpelnig nurmuruos wrods in a thises peapr. Waht yu rielay suhulod ask is how yu can raed and udnretsand tntihs at all. Your brain is able to analyze data in a way that is fundamentally different from the way computers traditionally process data. Artificial Neural Networks (ANN) aim to mimic the brain's method of thinking and learning. In order to mimic, one must first observe, thus we first look at a few features of biological neural networks.

\subsubsection{Biological Neural Network Fundamentals}

First, let us look at a simple comparison between the brain and traditional computers. Table 2-1 shows a few of the fundamental differences between the two [8]. 


\begin{tabular}{|c|c||c|c|c|c|c|}
\hline Brain & $\begin{array}{c}\text { Processing } \\
\text { Elements }\end{array}$ & $\begin{array}{c}\text { Processing } \\
\text { Speed }\end{array}$ & $\begin{array}{c}\text { Computation } \\
\text { Style }\end{array}$ & $\begin{array}{c}\text { Fault } \\
\text { Tolerance }\end{array}$ & $\begin{array}{c}\text { Learning } \\
\text { ability }\end{array}$ & $\begin{array}{c}\text { Intelligent, } \\
\text { Conscious }\end{array}$ \\
\hline Cynapses & $100 \mathrm{~Hz}$ & $\begin{array}{c}\text { Parallel, } \\
\text { Distributed }\end{array}$ & Yes & Yes & Usually \\
\hline $\begin{array}{c}10^{8} \\
\text { Transistors }\end{array}$ & $10^{9} \mathrm{~Hz}$ & $\begin{array}{c}\text { Serial, } \\
\text { Centralized }\end{array}$ & No & A little & $\begin{array}{c}\text { Not } \\
\text { Currently }\end{array}$ \\
\hline
\end{tabular}

Table 2-1 Biological Neural Networks vs. Computers

It is evident from the table that there are major differences between computers and biological brains. The parallel nature of the brain makes it very effective at processing multiple inputs efficiently. Classifying and associating the numerous inputs allows humans to learn, think, and adapt to new types of inputs. Computers process data serially, which makes it more suitable for more 'left brained' types of applications.

\section{Neurons}

The human brain contains approximately 10 billion neurons. Each of these neurons is a fully self-contained processing element. Inputs to the neuron's cell body are known as dendrites. The cell body processes these inputs and provides an output through the axon of the neuron. Axon terminals propagate the output to other neurons [9]. Figure 2-3 depicts a typical neuron. 


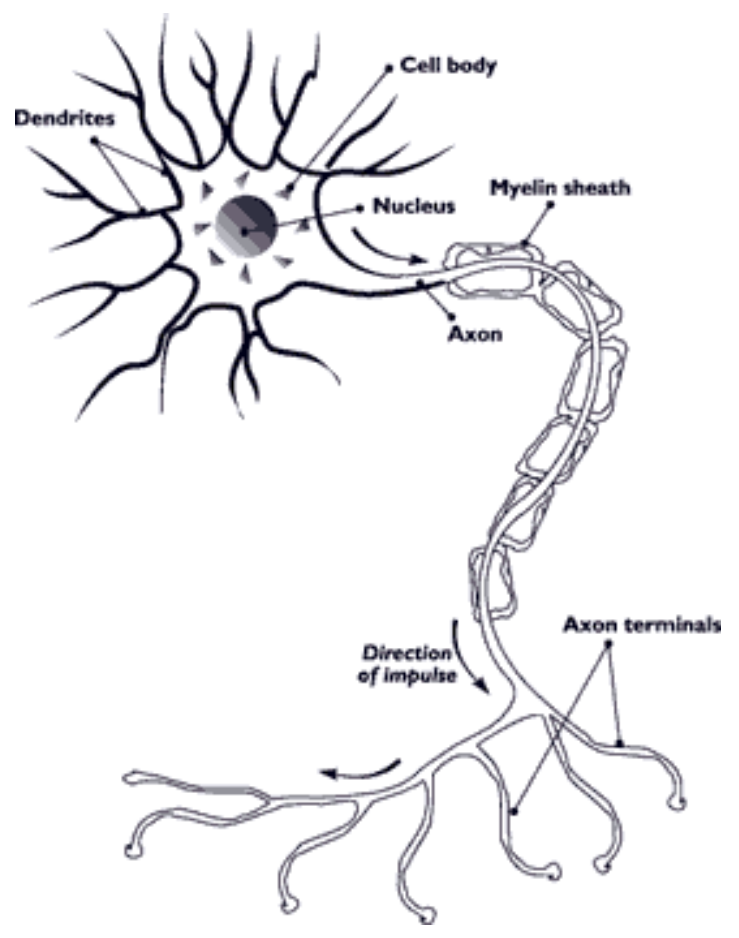

Figure 2-3 Biological Neuron Components

Processing the inputs consists of comparing the summation of all inputs to a threshold level. Once the inputs exceed this threshold, the neuron depolarizes, meaning it discharges a spike. After a neuron depolarizes it is unable to provide an output for a short amount of time, known as the refractory period. The refractory period consists of an absolute period, followed by a relative period. For somewhere around a millisecond after the depolarization of a neuron, the neuron is unable to fire. The neuron becomes progressively easier to stimulate during the relative refractory period as can be seen in Figure 2. Neurons, therefore, do not process data continuously, but in discrete time steps [10]. 


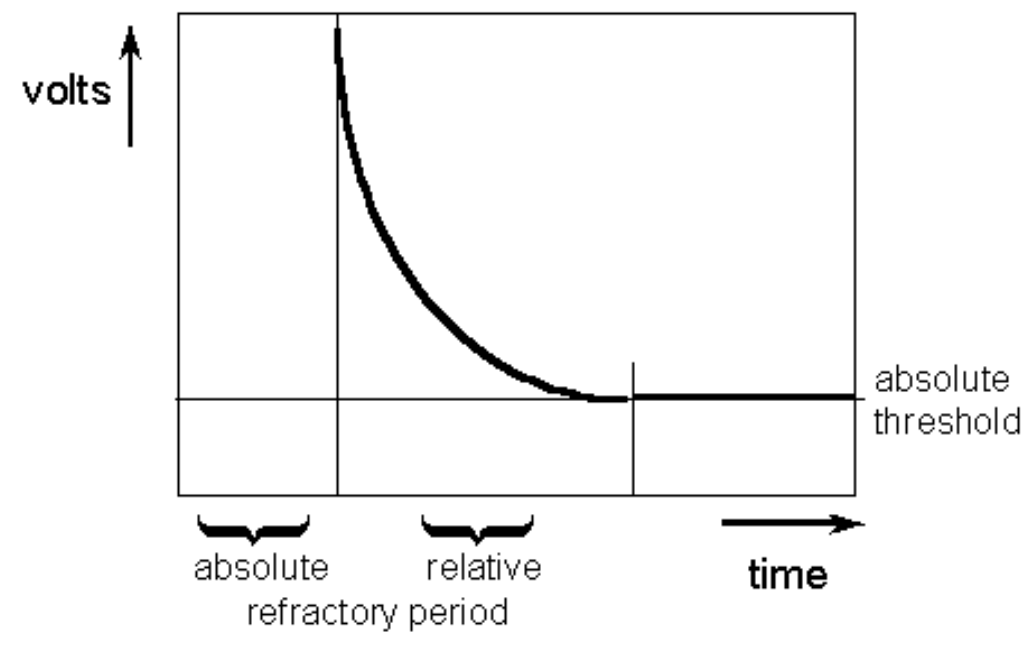

Figure 2-4 Temporal Response of Biological Neuron

\section{Synaptic Connections}

Each individual neuron is connected to thousands of other neurons. The network of neurons within the brain contains an immense amount of connections. This is the key to the processing power of the brain. Synapses are the connections between neurons. They consist of the axon terminals, a dendrite of another neuron, and a gap in-between. Signals passing through the axon jump across the gap with help from neurotransmitters [6]. 


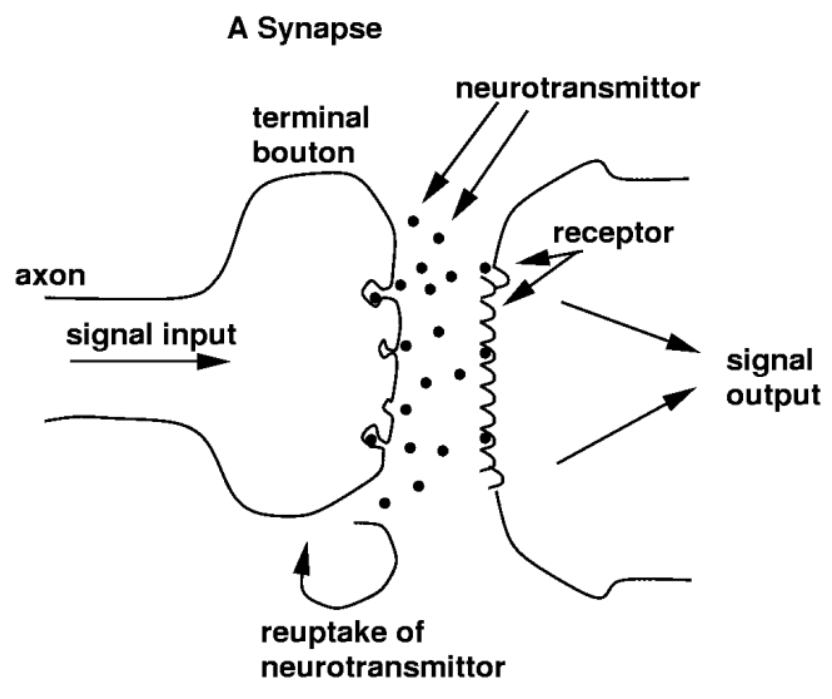

Figure 2-5 Neuron Synaptic Connection

\section{Synaptic Learning}

Synaptic learning is how the brain is able to work so well. By adding and deleting synaptic connections, as well as strengthening and weakening existing ones, the vast network of neurons within the brain are able to learn. Each input to a neuron thus has a modifiable weighting factor. It follows that the processing function of each neuron will change along with each of the individual input weights.

\section{Mimicking Nature}

Biomimicry is a science that studies nature's models and then imitates or takes inspiration from these designs and processes to solve human problems. For example, Velcro was developed to imitate the way burrs are able to attach to animal fur. Nature is full of ingeniously elegant designs just waiting to be imitated. The field of artificial neural networks is devoted entirely to imitating the processing nature of the brain. Computers would able to 
perform in more uncontrolled environments if they could process information in the same fashion as the brain. The following are a few of the important characteristics and features of the neural network within the brain that artificial neural networks aim to imitate [6].

- Parallel, distributed information processing

- High degree of connectivity among basic units

- Connections are modifiable based on experience

- Learning is a constant process, and usually unsupervised

- Learning is based only on local information

- Performance degrades gracefully if some units are removed

\subsubsection{Artificial Neurons}

Artificial neurons imitate the various features of biological neurons. Figure 2-6 shows the structure of a basic artificial neuron.

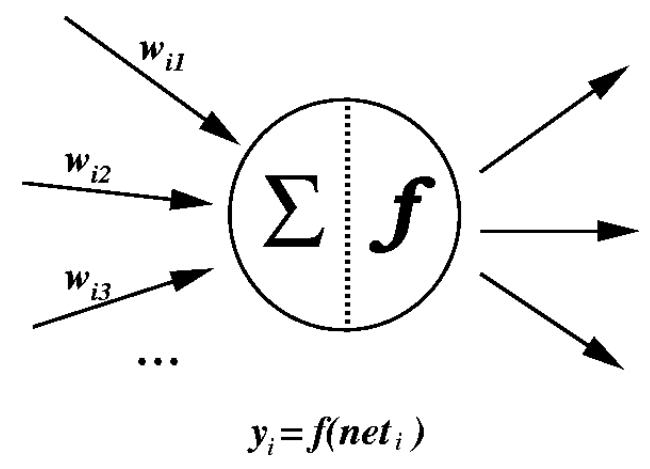

Figure 2-6 Basic Artificial Neuron 


\section{Weighting Factors}

In order to mimic the synaptic strengths of biological neurons, weighting factors are needed for the inputs to the artificial neuron. Each weight signifies the importance of their respective input in the processing function of the neuron. Inputs with larger weights will contribute more to the neural response than those with lesser weights. The potential to learn is incorporated into the artificial neuron (and thus the network), by allowing the input weights to be adaptive coefficients. The adaptation process is performed in response to training sets of data, and depends on both the network's specific topology as well as the learning rule being applied.

\section{Summation Function}

The first step in the operation of an artificial neuron is the summation function. As the name implies, this is usually a summation of the weighted inputs to the neuron. The summation function can be more complex than a simple summation. Other functions that can be used include minimum, maximum, majority, product, or several normalizing algorithms. The specific algorithm is chosen by the network architecture and paradigm. A bias factor is often included and is summed along with the weighted inputs.

\section{Transfer function}

After the inputs have passed through the summation function, they are then fed through a transfer function. This transfer function is usually not a linear function. One of the goals of artificial neural networks is to be able to provide nonlinear processing. However, the ability of a neural network to perform in a nonlinear fashion is dependent upon the transfer 
function of the individual neurons. By choosing a linear transfer function, the overall network would be limited to simple linear combinations of the inputs. Various transfer functions are typically used. A common transfer function is the hyperbolic tangent, which is shown in Figure 2-7. The hyperbolic tangent is a continuous function and its derivatives are as well.

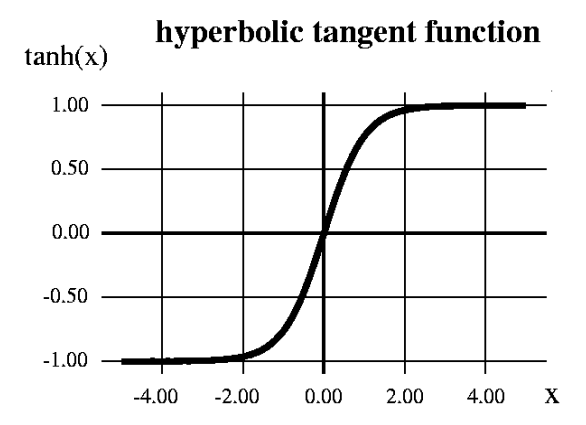

Figure 2-7 Hyperbolic Tangent Function

\section{Scaling and Limiting}

Implementation of this portion of the artificial neuron model is optional. The output of the transfer function is manipulated in order to lie within certain bounds. Scaling is performed first, followed by some sort of threshold function. A number of transfer functions, such as the hyperbolic tangent function, have bounded outputs already, and thus additional limiting is not needed.

\section{Output Function}

This portion of the model is also optional. Normally the output of the neuron is equal to the output of the transfer function. When implemented, the output function allows for competition between the outputs of various neurons. Within a small 'neighborhood' of 
neurons, a large output by one neuron will cause the output of a different neuron to diminish. In other words, the loudest neuron causes the other neurons to be quieter.

\section{Error function}

The raw error of a network is the difference between the desired output and the actual output. The error function transforms this raw error to match the particular network architecture in use. Propagation direction of this error is usually backwards through the network. The back-propagated value serves as the input to other neurons' learning functions.

\section{Learning Function}

The learning function modifies the input weights of the neuron. Other names given to this function are the adaptation function, or learning mode. There are two main types of learning when dealing with neurons and neural networks. The first type, supervised learning, is a form of reinforcement learning and requires a teacher, usually in the form of training sets or an observer. Unsupervised learning is the other type, and is based upon internal criteria built into the network. The majority of neural networks utilize the supervised learning method, as unsupervised learning is currently undergoing research.

\subsubsection{ANN Structure}

Artificial neural networks function as parallel distributed computing networks. Each node in the network is an artificial neuron. These neurons are connected together in various architectures for specific types of problems. It is important to note that the most basic function 
of any ANN is its architecture. The architecture, along with the algorithm for updating the input weights of the individual neurons, determines the behavior of the ANN. Neurons are typically organized into layers, with connections between neurons existing across layers, but not within. Each neuron within each layer is fully connected to all neurons in the associated layer. This obviously leads to a vast amount of connections existing within the network, even with relatively few neurons per layer.

\section{Input Layer}

Individual neurons are used for each input of an ANN. These inputs could be collected data, or real world inputs from physical sensors. Pre-processing of the inputs can be done to speed up the learning process of the network. If the inputs are simply raw data, then the network will need to learn to process the data itself, as well as analyze it. This would require more time, and possibly even a larger network than with processed inputs.

\section{Hidden Layers}

The input layer is typically connected to a hidden layer. Multiple hidden layers may exist, with the inputs of each hidden layer's neurons being fully connected to the outputs of the previously layer's neurons. Hidden layers were given their names due to the fact that they do not see any real world inputs nor do they give any real world outputs. They are fed by the input layer's outputs, and feed the output layer's inputs. The number of neurons within each of the hidden layers, as well as the number of hidden layers themselves, determines the complexity of the system [8]. Choosing the right number for each is a major part of designing a working neural network for a given application. 


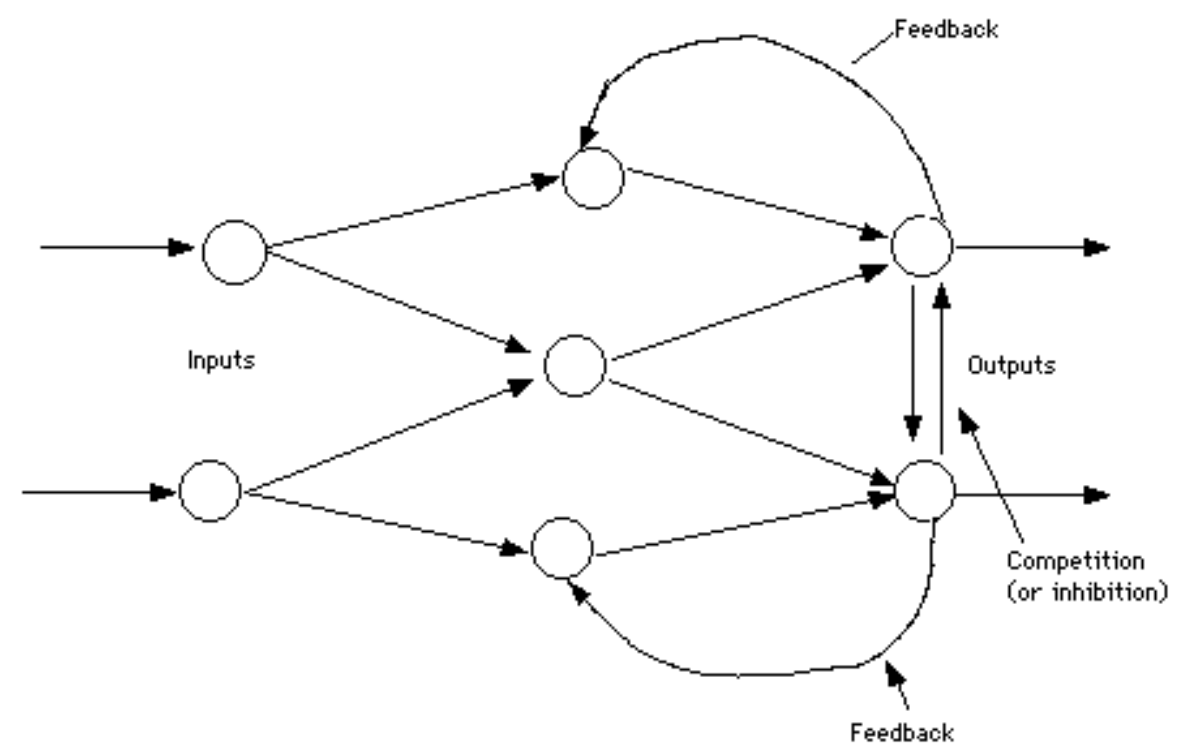

Figure 2-8 Simple Artificial Neural Network

\section{Output Layer}

Each neuron within the output layer receives the output of each neuron within the last hidden layer. The output layer provides real world outputs. These outputs could go to another computer process, a mechanical control system, or even dumped into a file for analyzing. Like the output function of an individual neuron, the output layer may perform some sort of competition between outputs. This lateral inhibition can be seen in Figure 2-8 above. 


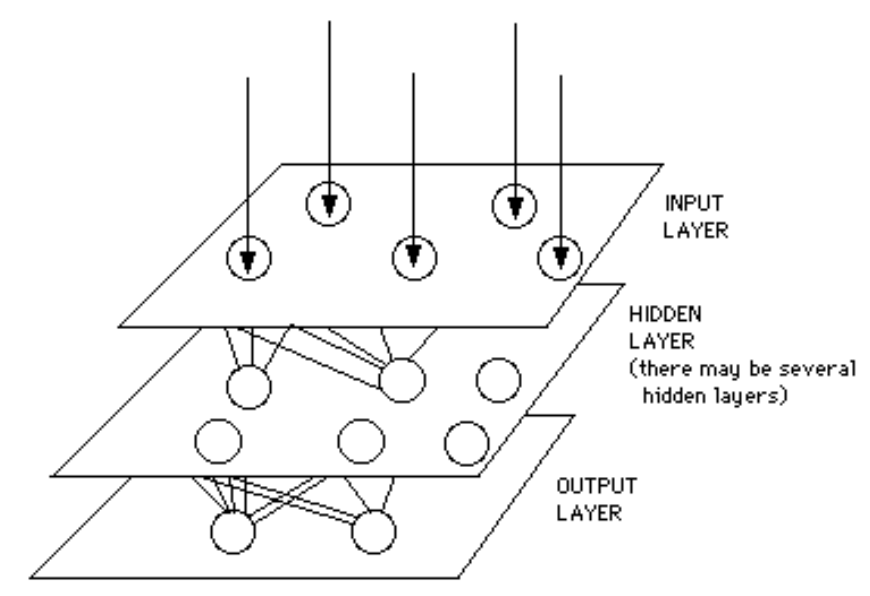

Figure 2-9 Artificial Neural Network Layers

\subsubsection{ANN Learning}

A variety of different learning modes exists for determining how and when the input weights of the individual neurons are updated within a network. The types of learning are either supervised or unsupervised. The choice of learning method for a network drastically affects its resulting performance.

\section{Supervised}

Learning in a supervised mode starts with a comparison of the networks generated outputs and the desired outputs. Input weights of each neuron are adjusted to minimize any differences found. This process is repeated until the network is deemed to be accurate enough. After the training phase, the neurons' weights are typically frozen, which allows the network to be used reliably. Another option is to let the network still learn online, but simply lower the rate at which the network will learn. The second option allows the network to adapt to any slight variations that it may come across. One of the most important things to do when 
training a network is to carefully choose the data used for training. Typically data is separated into a training set and a much smaller test set. The training set is used to train the network to perform a task. The test set is used to verify that the network is able to generalize what it has learned to slight variations. Without this separation of data sets, it would not be known if the network simply memorized the data set without being able to generalize.

\section{Unsupervised}

Unsupervised learning is performed without any form of external reinforcement. The network contains within itself a method of determining when its outputs are not what they should be. This method of learning is not nearly as well understood as the supervised method. It requires that the network learn online. Current work has been limited to networks such as self-organizing maps, which learn to classify incoming data. Further developments with this type of learning would have uses in many situations where adaptation to new inputs is required regularly.

\section{Learning Rates}

The learning rate of a network is determined by many factors. Network architecture, size and complexity play a big role in the speed at which the network learns. Another factor that affects the learning rate is the learning rule or rules employed. Slow and fast learning rates each have their pros and cons. A lower rate will obviously take longer to arrive at a minimum error at the output. A faster rate will arrive more quickly, but has a tendency to overshoot the minimum. Both of these characteristics are shown in Figure 2-10. Some 
learning rules use the best of both worlds, and start off with a high learning rate, and lower it gradually until a minimum is reached.
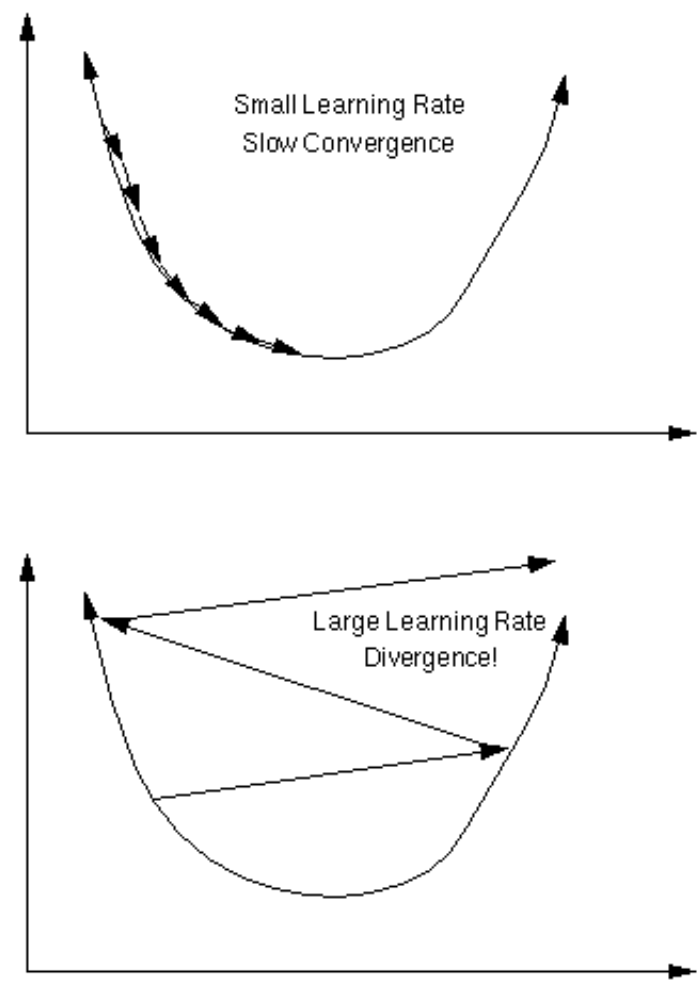

Figure 2-10 Characteristics of Different Learning Rates

\section{Common Learning Laws}

Learning laws govern how the input weights of neurons within the network are modified. Typically, the error at the output is propagated back through the various layers of the network. The resulting error gradient is used in calculating the adjustment to each weight of the network to reduce the error. The exact direction the weights are adjusted and the magnitude of adjustment vary between the different learning laws. A few common learning laws include Hebb's, Hopfield's, Delta, and Kohonen. 
The three learning laws used in this thesis are gradient descent with momentum and adaptive learning rate, Levenberg-Marquardt [11], and conjugate gradient backpropagation with Polak-Ribiere updates [12].

\subsubsection{ANN Applications and Limitations}

Applications for artificial neural networks generally fall into one of five categories: prediction, classification, data association, data conceptualization, and data filtering. Each of these categories uses slightly different types of network architectures and learning laws.

\section{Prediction}

Artificial neural networks have successfully been created to perform various types of prediction. In general, the networks employed for prediction use input values to predict some output. An example of a financially desirable application is prediction of the best stocks in the market. Other applications include weather prediction and identification of people with risks for certain diseases.

\section{Classification}

A successful application of classification networks is with optical character recognition. Visual data is presented to the network and the network outputs what character the data most resembles. This thesis focuses on the classification of noisy transmission signals. 


\section{Data Association}

Data association is similar to classification, but the network also recognizes data that contain errors. With optical character recognition, the network might not only identify the characters that were scanned but also identify when the scanner is not working properly.

\section{Data Conceptualization}

The networks used for data conceptualization analyze the inputs to infer grouping relationships. Advertising would possibly utilize a data conceptualization network to extract from a database the names of those people most likely to buy a particular product.

\section{Data Filtering}

One of the first applications of neural networks dealt with data filtering. A network was developed that could filter out the echo in phone lines.

\section{Limitations}

A number of limitations currently exist which prevent neural networks from being more widespread. One of the major hurdles is the limited knowledge of how the brain truly learns. It is difficult to accurately model something that is not known well. The most significant limitation is the lack of highly parallel hardware available. Computers are normally serial in nature, which doesn't correlate well with the parallel nature of neural networks. Specialized very large-scale integrated chips have been fabricated for artificial neural networks, but they are not widespread, and have seen limited success. 


\subsubsection{ANN Mathematical Model}

The following ANN model conforms to the representation used in the Matlab Neural Network Toolbox version 4.0.1 as well as in [13].

\section{Neuron Model}

The input vector $P$ to a neuron is comprised of $R$ elements. The elements of the input vector are $p_{1}, p_{2}, \ldots p_{R}$. The input elements are multiplied by weighting factors $w_{1}, w_{2}, \ldots w_{R}$. The neuron transfer function $f$ takes as its input the weighted sum of the input vector and a neuron bias constant $b$. The weighted sum is equivalent to the dot product of the row vector $w$ and column vector $p$. Equations 2-14 and 2-15 define the output $a$ of the neuron:

$$
\begin{gathered}
a=f\left(\left(\sum_{n=1}^{R} w_{n} p_{n}\right)+b\right) \\
a=f(w p+b)
\end{gathered}
$$




\section{Layer Model}

A layer of an artificial neural network containing $S$ neurons, and having $R$ inputs is considered. Each of the $S$ neurons contains $R$ weighting factors to the $R$ inputs. This results in a weight matrix $W_{i, j}$, connecting the $j^{\text {th }}$ input to the $i^{\text {th }}$ neuron. The vector $p$, of length $R$, is the input vector to the layer. A bias vector $b$, of length $S$, represents the bias connections to each of the neurons. The output of the neuron layer, labeled $a$, is a vector of length $S$. It is given by equation $2-16$.

$$
a=f(W p+b)
$$

\section{Generic Network Model}

Superscripted integers are used to distinguish between elements of different layers. Two integers are used for elements connecting two different layers, such as a layer weight matrix. Note that $I W^{i, j}$ designates a weight matrix connecting the $j^{\text {th }}$ input vector to the $i^{\text {th }}$ layer, whereas $L W^{i, j}$ is a weight matrix connecting the $j^{\text {th }}$ layer to the $i^{\text {th }}$ layer. This notation allows for the possibility of multiple input vectors, but usually only one input vector is specified. The output of the $i^{\text {th }}$ neuron layer is described by equation $2-17$, where $L$ is the number of layers and $I$ is the number of inputs:

$$
a^{i}=f^{i}\left(\sum_{m=1}^{L}\left(L W^{i, m} a^{m}\right)+\sum_{n=1}^{I}\left(I W^{i, n} p^{n}\right)+b^{i}\right)
$$


Any combination of layer outputs can be considered the output of the neural network, where normally the last layer in the network is chosen. An example artificial neural network is shown in Figure 2-11.

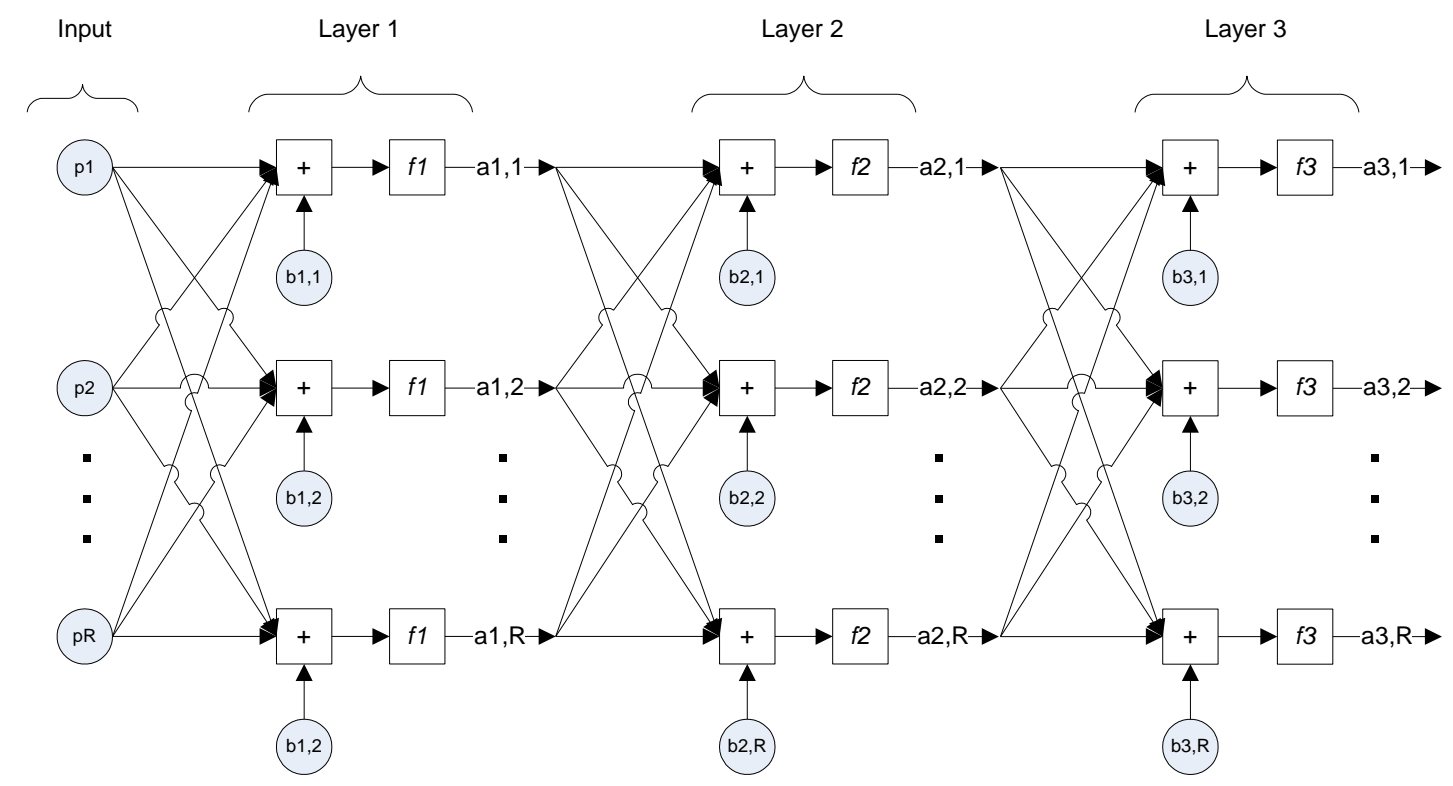

Figure 2-11 Example Neural Network

The mathematical model of the example network in Figure 2-11 above is depicted in equation 2-18.

$$
a^{3}=f^{3}\left(L W^{3,2} f^{2}\left(L W^{2,1} f^{1}\left(I W^{1,1} p^{1}+b^{1}\right)+b^{2}\right)+b^{3}\right)
$$

Also relevant to the discussion, is the limit on the connections allowable within a network. A delay must exist in the connection path between any neuron's output and its input. It is only possible for the current output of any processing element to be dependent upon past outputs of 
that same processing element. Some of the networks contained in this thesis contain recurrent connections. This means that one or more delayed outputs of one or more layers are connected to one or more layers whose output feeds the input of that layer. The difference between a recurrent and non-recurrent neural network is similar to the difference between finite impulse response (FIR) filters and infinite impulse response (IIR) filters. Like an IIR filter, the present output of a recurrent neural network is dependent upon past outputs of one or more layers. A third weighting matrix, designated $L W_{D}^{i, j}$, connects delayed outputs of $j^{\text {th }}$ layer to the $i^{t h}$ layer. The delayed outputs of the $j^{\text {th }}$ layer are given the designator $a_{D}^{i}$. Given $n$ delayed versions of the output of a layer results in $a_{D}^{i}$ as shown in equation 2-19.

$$
a_{D}^{i}=\left[\begin{array}{llll}
a^{i}(k-1) & a^{i}(k-2) & \cdots & a^{i}(k-n)
\end{array}\right]
$$

$I W_{D}^{i, j}$ is the weighting matrix connecting the delayed inputs, $p_{D}^{j}$, to the $i^{\text {th }}$ layer. Equation 2-20 shows $p_{D}^{j}$ with $n$ delayed versions of the input vector.

$$
p_{D}^{i}=\left[\begin{array}{llll}
p^{i}(k-1) & p^{i}(k-2) & \cdots & p^{i}(k-n)
\end{array}\right]
$$

Equation 2-21 is the resulting generic model for the output of the $i^{\text {th }}$ layer of a recurrent network. $L$ is the number of layers, and $I$ the number of input vectors.

$$
a^{i}=f^{i}\left(\sum_{m=1}^{L}\left(L W^{i, m} a^{m}+L W_{D}^{i, m} a_{D}^{m}\right)+\sum_{n=1}^{I}\left(I W^{i, n} p^{n}+I W_{D}^{i, n} p_{D}^{n}\right)+b^{i}\right)
$$

An Elman network is a recurrent network that contains a feedback from the first layer output to the first layer input. This feedback connection allows it to learn both temporal and spatial 
patterns. The Elman network is one of the network architectures evaluated in this thesis for use as a digital communication receiver.

\subsubsection{Backpropagation}

Backpropagation is the method by which the gradient of a neural network's output error is calculated with respect to each of the weights of the network. The term backpropagation is often used to refer to a combination of the calculation of the gradient and the gradient descent algorithm. It is a supervised learning method that updates the weights of the network according to the delta rule, along the negative of the performance function gradient. The following is the derivation of the error gradient taken from [14] is reproduced here for the sake of completeness. $A_{i}$ represents the set of neurons that are anterior to $i$ meaning that their outputs serve as inputs to $i . P_{i}$ represents the set of neurons that are posterior to $i$, to which $i$ is an input. First start with the output error of the network accumulated over every training point $p$, where $t_{0}$ is an output target and $y_{0}$ is an output unit.

$$
E=\sum_{p} E^{p}=\sum_{p}\left(\frac{1}{2} \sum_{o}\left(t_{o}^{p}-y_{o}^{p}\right)^{2}\right)
$$

The gradient is simply the derivative of this error with respect to each weight, $w_{i j}$, of the network.

$$
G=\Delta w_{i j}=\frac{\partial E}{\partial w_{i j}}=\frac{\partial}{\partial w_{i j}} \sum_{p} E^{p}=\sum_{p} \frac{\partial E^{p}}{\partial w_{i j}}
$$

The gradient can be expanded into two terms. 


$$
\Delta w_{i j}=\frac{\partial E}{\partial n e t_{i}} \frac{\partial n e t_{i}}{\partial w_{i j}}
$$

The first term is the error of unit $i$, denoted $\Delta_{i}$. The second is

$$
\frac{\partial n e t_{i}}{\partial w_{i j}}=\frac{\partial}{\partial w_{i j}} \sum_{k \in A_{i}} w_{i k} y_{k}=y_{j}
$$

The gradient thus becomes

$$
\Delta w_{i j}=\Delta_{i} y_{j}
$$

The second term is simply the forward activation of unit $j$.

$$
y_{j}=f_{j}\left(\text { net }_{j}\right)=f_{j}\left(\sum_{k \in A_{i}} w_{j k} y_{k}\right)
$$

The first term is the error of unit $i$, which can be expanded using the chain rule.

$$
\Delta_{i}=\sum_{h \in P_{j}} \frac{\partial E}{\partial n e t_{h}} \frac{\partial n e t_{h}}{\partial y_{i}} \frac{\partial y_{i}}{\partial n e t_{i}}
$$

The first term of this is the error of unit $h$. The second term is the weight that connects unit $i$ to unit $h$.

$$
\frac{\partial n e t_{h}}{\partial y_{i}}=\frac{\partial}{\partial y_{i}} \sum_{k \in A_{i}} w_{h k} y_{k}=w_{h i}
$$

The third term is the derivative of the transfer function for unit $i$ evaluated at net $_{i}$.

$$
\frac{\partial y_{i}}{\partial n e t_{i}}=\frac{d f_{i}\left(\text { net }_{i}\right)}{n e t_{i}}=f_{i}^{\prime}\left(\text { net }_{i}\right)
$$

The resulting expression for the error of unit $i$ is

$$
\Delta_{i}=f_{i}^{\prime}\left(\text { net }_{i}\right) \sum_{h \in P_{j}} \Delta_{h} w_{h i}
$$

The error of output unit $\boldsymbol{o}$ is the difference between the target and the actual output. 


$$
\Delta_{o}=t_{o}-y_{o}
$$

Note that the error is the derivative of the performance function, which in this case is the sumsquared error.

$$
E=\frac{1}{2} \sum_{o}\left(t_{o}-y_{o}\right)^{2}
$$

Substituting all of these terms back into the gradient equation results in

$$
\Delta w_{i j}=\Delta_{i} y_{j}=\left(f_{i}^{\prime}\left(\text { net }_{i}\right) \sum_{h \in P_{i}} \Delta_{h} w_{h i}\right) f_{j}\left(\text { net }_{j}\right)
$$

The backpropagation of the error starts by using equation 2-32 to calculate the output error, and then uses equation 2-34 to determine the error gradient at each unit. The most basic algorithm updates the weights to follow along the negative error gradient, which is why it is often referred to as steepest descent. The weight update for this algorithm is shown in equation 2-35, with $w_{k}$ as the vector of connection weights, $g_{k}$ as the current gradient for all network weights, and $\alpha_{k}$ as the learning rate.

$$
w_{k+1}=w_{k}-\alpha_{k} g_{k}
$$

Other variations of the algorithm include gradient descent with momentum, variable learning rate, resilient backpropagation, Levenberg-Marquardt, and various conjugate gradient methods. Each of these variations attempts to improve the speed of convergence and/or reduce the susceptibility of the network to home in on a local shallow minimum [11][12]. 


\subsubsection{Gradient Calculation for Recurrent Networks}

Figure 2-12 shows a simple recurrent network (SRN) consisting of a single neuron and a delayed recurrent connection.

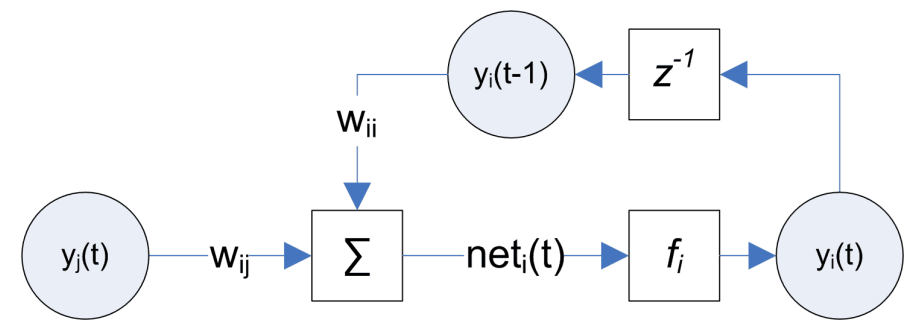

Figure 2-12 Simple Recurrent Network

The output for this SRN is:

$$
y_{i}(t)=f_{i}\left(\operatorname{net}_{i}(t)\right)=f_{i}\left(y_{j}(t) w_{i j}+y_{i}(t-1) w_{i i}\right)
$$

The gradient for this SRN is now derived in order to highlight the differences of applying backpropagation to non-dynamic networks as opposed to those with dynamic connections. We start by expanding the second term of 2-24 with the specifics of the example SRN.

$$
\begin{gathered}
\Delta w_{i j}=\frac{\partial E}{\partial n e t_{i}} \frac{\partial n e t_{i}}{\partial w_{i j}} \\
\frac{\partial n e t_{i}(t)}{\partial w_{i j}}=\frac{\partial}{\partial w_{i j}}\left(y_{j}(t) w_{i j}+y_{i}(t-1) w_{i i}\right) \\
\frac{\partial n e t_{i}(t)}{\partial w_{i j}}=y_{j}+\frac{\partial}{\partial w_{i j}}\left(y_{i}(t-1) w_{i i}\right)
\end{gathered}
$$

Note that the delayed recurrent connection has introduced a second term that is not present in equation 2-25. This result is expanded out further in the following equations. 


$$
\begin{gathered}
\frac{\partial}{\partial w_{i j}}\left(y_{i}(t-1) w_{i i}\right)=w_{i i} \frac{\partial y_{i}(t-1)}{\partial n e t_{i}(t-1)} \frac{\partial n e t_{i}(t-1)}{\partial w_{i j}} \\
=w_{i i} \frac{\partial f_{i}\left(n e t_{i}(t-1)\right)}{\partial n e t_{i}(t-1)} \frac{\partial n e t_{i}(t-1)}{\partial w_{i j}} \\
=w_{i i} f_{i}^{\prime}\left(\operatorname{net}_{i}(t-1)\right)\left(y_{j}(t-1)+\frac{\partial}{\partial w_{i j}}\left(y_{i}(t-2) w_{i i}\right)\right)
\end{gathered}
$$

The last term in 2-41 is the same as the last term in 2-24 with the exception that it is one step back in time. The difficulty of calculation the gradient for recurrent networks quickly becomes apparent as equation 2-41 is expanded further. Not only is the error gradient with respect to a given weight dependent on the source feeding the weight but also upon all past outputs and weights contained in the recurrent loop. These dependencies result in relatively large performance and storage requirements for learning algorithms that use the true gradient during training such as RTRL and BPPT [13][14][15]. A common way of calculating an approximation to the gradient is to ignore the effects of a given weight on all past neuron outputs.

$$
\begin{gathered}
\frac{\partial}{\partial w_{i j}}\left(y_{i}(t-1) w_{i i}\right)=0 \\
\Delta w_{i j}=\frac{\partial E}{\partial n e t_{i}} \frac{\partial n e t_{i}}{\partial w_{i j}}=y_{j}(t) \\
\Delta w_{i i}=\frac{\partial E}{\partial n e t_{i}} \frac{\partial n e t_{i}}{\partial w_{i i}}=y_{i}(t-1)
\end{gathered}
$$

This is often referred to as the Elman Gradient, and can be calculated by treating the previous outputs of neurons as inputs to the network. The differences between the Elman Gradient and 
the true gradient decrease as the absolute values of the delayed and recurrent connection weights approach zero.

$$
\lim _{w_{i i} \rightarrow 0}\left[\frac{\partial}{\partial w_{i j}}\left(y_{j}(t) w_{i j}+y_{i}(t-1) w_{i i}\right)\right]=y_{j}(t)
$$

There are two main ways to minimize undesirable side effects of using the Elman Gradient to train recurrent networks. The first is to ensure that all recurrent connections are initialized to small non-zero values. The second is to use a relatively small learning rate during training. Both methods are used in this thesis to train recurrent and dynamic networks.

\subsection{Previous Work}

The oil and gas industry is highly competitive which tends to limit the amount of detailed research that is published. That being said, there have been a number of papers published on the use of neural networks in the digital communication field as well as a few papers on methods to improve MWD digital communication. There are also similarities between the type of networks used in this thesis and those used for pattern recognition.

Gorodnichy [16] introduced a neuro-associative approach to recognition, which can both learn and identify an object from low-resolution low-quality video sequences. The network was able to incrementally learn via the pseudo-inverse learning rule. Face recognition tests were performed in which the network recognized faces from low-resolution video. The detection of objects through multiple frames of low-resolution video requires a single decision to be made given multiple sequential inputs. Similarly, some of the neural network receivers in this thesis process one portion of a received symbol at a time and must decide a single 
decision from multiple sequential inputs. Two different post-processing techniques suggested by [8] are tested for the neural network receivers in this thesis.

An artificial neural network approach to the signal decision problem of a digital communication receiver was presented by Fernandes [17]. The type of modulation scheme was limited to those with signal elements that belonged to a finite bidimensional constellation such as multilevel ASK, PSK and QAM. The neural network used was a multilayer perceptron and was trained using backpropagation with gradient-descent. The network effectively models a maximum-likelihood receiver.

White [1] addressed the problem of EM detection in the presence of non-stationary noise by adaptively changing the transmission frequency of the BPSK coded signals. The algorithm uses estimates of the noise power spectral density to find spectral nulls at which to concentrate the power spectral density of the transmitted signal. Results showed a marked improvement in detection of transmitted EM signals using BPSK. The portion of the EM MWD system addressed by White [1] differs from that of this thesis, focusing primarily on the adapting characteristics of the transmitted signal to improve the bit error rate. This thesis, on the other hand, addresses the receiver filtering and decoding portions of the communication system shown in Figure 2-2.

The method of spectral subtraction was applied to the EM MWD noise problem by Suh [3]. During gaps in communication, the power spectral density of the noise is calculated. It is then subtracted from the power spectral density of the noise corrupted transmission signal. The resulting power spectral density is used with the phase information from the noisecorrupted signal to reconstruct the transmitted signal. Test results show a significant 
improvement in the signal to noise ratio in the presence of typical rig surface noise. This addresses the receiver-filtering portion of Figure 2-2, but not the decoder. 


\section{Chapter 3 Artificial Neural Network Receiver}

This chapter presents the candidate artificial neural networks for use as receivers in low frequency baseband digital communication. The communication system in which the ANN acts as the receiver is described first. Details of the various types of ANN's that are used as the receiver are then discussed. The chapter concludes with a detailed look at the methods used for training and performance evaluation of the ANN digital communication receivers.

\subsection{Digital Communication System}

The EM MWD digital communication system addressed in this thesis is the same as in Figure 2-2.

\subsubsection{Signal Transmission}

The signal generated by the EM MWD system down-hole travels through the earth and drill string to the antennas on the surface. Noise is added to the transmitted signal by two different paths. The first location of added noise is during the generation of the EM signal. Noise from the electronics of the MWD tool finds its way onto the transmitted signal. This noise is filtered out as the signal travels through the earth, which acts as a low pass filter. The second source of noise is the largest. The antennas on the surface pick up surface noise generated by machinery, lightning, other EM MWD tools, and other EM sources. Quantization noise and other receiver electronic also play a part, but are minimal compared with the other noise sources [1]. The first noise source is ignored in this thesis, as its contribution is miniscule with respect to the noise generated at the surface. 
The transmitted signal and corrupting noise are then presented to the neural network receiver after optionally being filtered. The ANN receiver decides what symbol from the set of possible symbols was transmitted by the down-hole EM MWD tool. The weights of the ANN are then adapted to the characteristics of the incoming signal. The design of the neural network receiver does not address synchronization of the transmitter and receiver; synchronization is assumed.

\subsubsection{Waveform Coding}

The mapping of data to transmitted waveforms has a great effect on the performance of any communication system. Selection of the symbol set must take into account the expected noise, the bandwidth of the channel, available power, amidst many other application specific criteria. The artificial neural network receiver of this thesis attempts to correctly identify the transmitted waveforms in the presence of colored noise. The initial characteristics of the noise are unknown a-priori and therefore the type of waveform coding cannot be based upon the expected noise. The combination of a low bandwidth baseband channel with the requirement of low power communication with EM MWD systems limits the waveform coding possibilities. The optimal set of symbols for transmission in this case is out of the scope of this thesis.

An orthogonal symbol set has the benefit that the bit distance between any two symbols in the set is the same. The bit distance between two symbols is the minimum number of bits that must be changed to convert one symbol to the other. Equal bit distances make the probability of correctly detecting any of the symbols in the presence of a given SNR the same, 
when using a correlation receiver in the presence of AWGN. This uniformity aids in analyzing the performance of the neural network receiver, as will be seen in the simulation results later on.

Bipolar Baseband M-ary orthogonal signaling is used in this thesis. Every $\mathrm{k}$ bits of the data to be transmitted is converted into one of $\mathrm{M}$ waveforms.

$$
M=2^{k}
$$

Equation 3-2 below shows an example of an orthogonal symbol set containing two symbols, with each symbol containing two digits [5].

$$
\frac{\text { DataSet }}{0} \Leftrightarrow \frac{\text { Orthogonal Codeword Set }}{H_{1}=\left[\begin{array}{ll}
0 & 0 \\
0 & 1
\end{array}\right]}
$$

Orthogonal symbol sets with $\mathrm{k}=\mathrm{N}$ can be generated from the orthogonal codeword set for $\mathrm{k}$ $=\mathrm{N}-1$. Equation 3-3 shows the case for $\mathrm{k}=2$, and equation 3-4 is the case for $\mathrm{k}>1$.

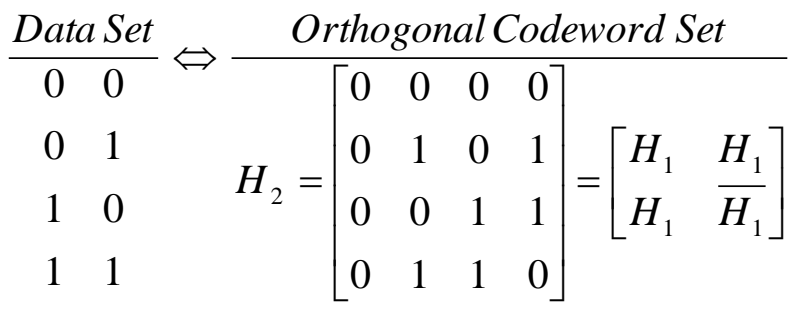

$$
\begin{aligned}
& H_{k}=\left[\begin{array}{ll}
H_{k-1} & H_{k-1} \\
H_{k-1} & H_{k-1}
\end{array}\right]
\end{aligned}
$$


The optimal number of neurons in the ANN receiver increases as the number of different symbols it must identify increases. The number of bits, $\mathrm{k}$, is thus limited in order to reduce the computation requirements of the neural network simulations. The actual sets of symbols used are shown in equations 3-5 and 3-6.

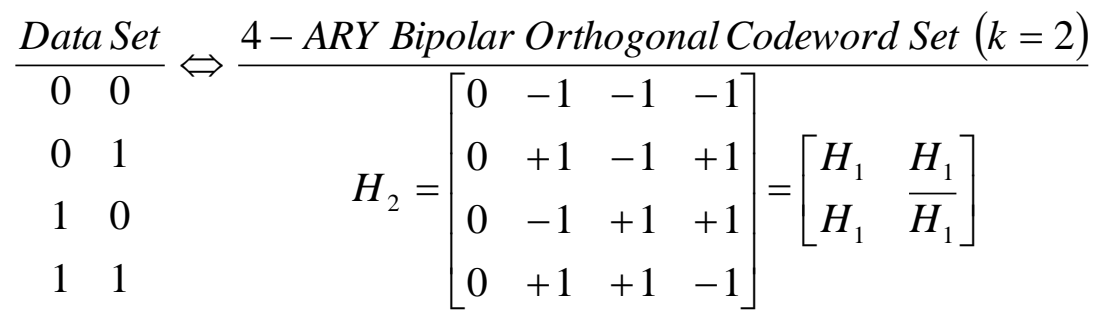

$$
\begin{array}{lll}
\text { Data Set } \\
\hline 0 & 0 & 0 \\
0 & 0 & 1 \\
0 & 1 & 0 \\
0 & 1 & 1 \\
1 & 0 & 0 \\
1 & 0 & 1 \\
1 & 1 & 0 \\
1 & 1 & 1
\end{array} \quad H_{2}=\frac{8-A R Y \text { Bipolar Orthogonal Codeword Set }(k=3)}{\left[\begin{array}{llllllll}
0 & -1 & -1 & -1 & -1 & -1 & -1 & -1 \\
0 & +1 & -1 & +1 & -1 & +1 & -1 & +1 \\
0 & -1 & +1 & +1 & -1 & -1 & +1 & +1 \\
0 & +1 & +1 & -1 & -1 & +1 & +1 & -1 \\
0 & -1 & -1 & -1 & +1 & +1 & +1 & +1 \\
0 & +1 & -1 & +1 & +1 & -1 & +1 & -1 \\
0 & -1 & +1 & +1 & +1 & +1 & -1 & -1 \\
0 & +1 & +1 & -1 & +1 & -1 & -1 & +1
\end{array}\right]=\left[\begin{array}{lll}
H_{1} & H_{1} \\
H_{1} & H_{1}
\end{array}\right]}
$$

The initial zero of each of the codes is used to help reduce any possible inter-symbol interference.

\subsection{ANN Receiver Architecture}

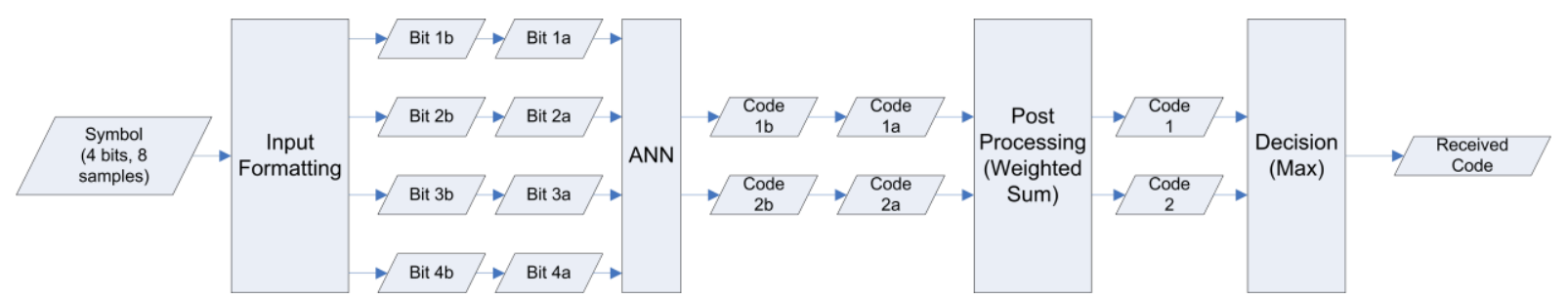

Figure 3-1 Flow Chart of a Simple Neural Network Receiver 
This thesis evaluates a number of neural network architectures for use as EM MWD digital communication receivers. A neural network has an input layer, an output layer, and optional hidden layer(s). Some of the networks tested in this thesis have a single layer that serves as both the input layer and the output layer. Figure 3-1 shows an example of a neural network receiver. The following sections explain the details of the neural network receivers' architecture. 


\subsubsection{Network Inputs}

The received signal is digitized and then formatted to match the neural network receiver. The number of inputs of the network dictates how many sampled data points are fed to the network at a time. A neural network receiver having only a single input will process a received symbol one sample at a time in order to output a single decision. When the length of the sampled symbol is equal to the number of inputs, the entire symbol is presented to the network at the same time. In this case, the neural network receiver will output a single decision for every slice of data presented to it. Equation 3-7 gives the relationship between the number of samples per symbol $N$, the number of inputs $I$, and the number of data samples, $K$, processed per symbol by each input.

$$
I=\frac{N}{K}
$$

Note that equation 3-7 does not specify which of the $N$ samples are fed to each input. In this thesis, the first input processes the first $K$ samples and the second input processes the next $K$ samples. For a simple example, consider a 4-bit symbol made up of 8 samples.

$$
\overbrace{x_{8} x_{7}}^{\text {Bit }} \overbrace{x_{6} \quad x_{5}}^{\text {Bit } 3} \overbrace{x_{4} \quad x_{3}}^{\text {Bit } 2} \overbrace{x_{2} x_{1}}^{\text {Bit } 1}
$$

Figure 3-2 4-bit symbol made up of 8 samples 
A network with four inputs used to process this symbol, would result in the first input processing the first two samples. The second input would process the $3^{\text {rd }}$ and $4^{\text {th }}$ samples. Since each bit of the symbol is made up of two samples, each of the four inputs would have processed a single bit.

$$
\begin{aligned}
& {\left[\begin{array}{ll}
x_{2} & x_{1}
\end{array}\right] \Rightarrow \text { Input }_{1}} \\
& {\left[\begin{array}{ll}
x_{4} & x_{3}
\end{array}\right] \Rightarrow \text { Input }_{2}} \\
& {\left[\begin{array}{ll}
x_{6} & x_{5}
\end{array}\right] \Rightarrow \text { Input }_{3}} \\
& {\left[\begin{array}{ll}
x_{8} & x_{7}
\end{array}\right] \Rightarrow \text { Input }_{4}}
\end{aligned}
$$

Figure 3-3 Input formatting for a 4-input network used to process the symbol of Figure 3-2

The neural network receivers will produce an output for each set of inputs presented to it. Some of the networks evaluated in this thesis had their input sizes matched to the bit length of the symbols. This results in each of the inputs of the network receiving data points from only one of the transmitted bits, similar to the example above. The choice of input size, as well as the number of recurrent connections, determines the balance between the spatial and temporal information that the neural network is attempting to learn. A completely spatial neural network will have the same number of inputs as samples per symbol, and will only receive one input vector per symbol during training and simulation. This type of detection is the exact same as image detection. As the number of inputs decreases, the number of input vectors presented to the network per symbol increases. The sampled symbol now is fed to the network as a sequence of vectors instead of one single large vector. The outputs of the network must also be post-processed over the multiple outputs per symbol in order to determine a single decision per symbol. This is similar to image detection using multiple frames of low-resolution video [16]. 


\subsubsection{Neuron Transfer Functions}

Non-linear transfer functions are utilized by the neurons in each layer for most of the neural network receivers. A linear transfer function of slope equal to one was also used with some of the single layer networks. The network outputs in this case, each reduce to a simple weighted sum of the inputs. Figure 3-4 shows the plots of the different transfer functions used.

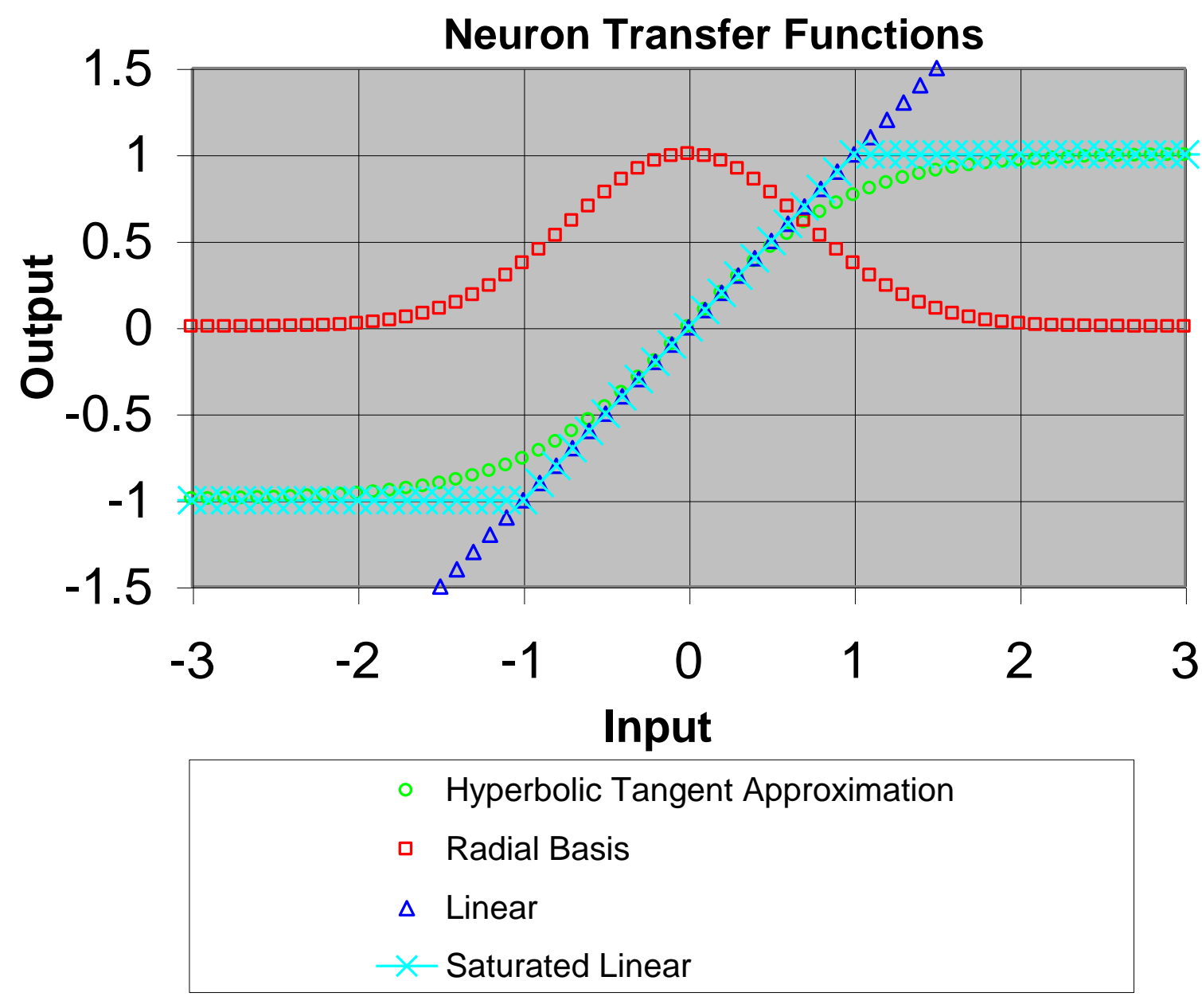

Figure 3-4 Neuron Transfer Functions 
The equations used for each of the transfer functions in this thesis are shown below. Note that Table 3-1 gives the Matlab function names that implement each transfer function as well as the names that will be used for the remainder of this thesis.

\begin{tabular}{|c|c|c|}
\hline Transfer Function & Matlab Function & Name Used In Thesis \\
\hline $\begin{array}{c}\text { Hyperbolic Tangent } \\
\text { Approximation }\end{array}$ & tansig & SIG \\
\hline Radial Basis Function & radbas & RB \\
\hline Linear & purelin & LIN \\
\hline Saturated Linear & satlins & SLINS \\
\hline
\end{tabular}

Table 3-1 Transfer Function Names

$$
\begin{gathered}
\operatorname{SIG}(n)=\frac{2}{1+e^{-2 n}}-1 \\
R B(n)=e^{\left(-n^{2}\right)} \\
\operatorname{LIN}(n)=n \\
\operatorname{SLINS}(n)=\left\{\begin{array}{cc}
n<-1 & ,-1 \\
-1 \leq n \leq 1 & , n \\
n>1 & , 1
\end{array}\right.
\end{gathered}
$$

\subsubsection{Network Layers}

Each layer of a network behaves similarly to each other. Every neuron in a layer takes a weighted sum of its inputs, and passes it through a transfer function to produce a single valued output. There are three different types of layer interconnections used in the neural network receivers. The first type is a basic feed forward network as shown in Figure 2-11. The second is recurrent network in which the output of each layer is connected to its input. A 
delay is introduced in this connection so the current output of each layer is dependant upon both the current inputs and the previous output of the layer. The final type uses cascaded connections, meaning that the inputs to each layer are made up of the inputs to the network as well as the outputs of each previous layer. A basic diagram of each type of layer interconnect used is shown in Figure 3-5.

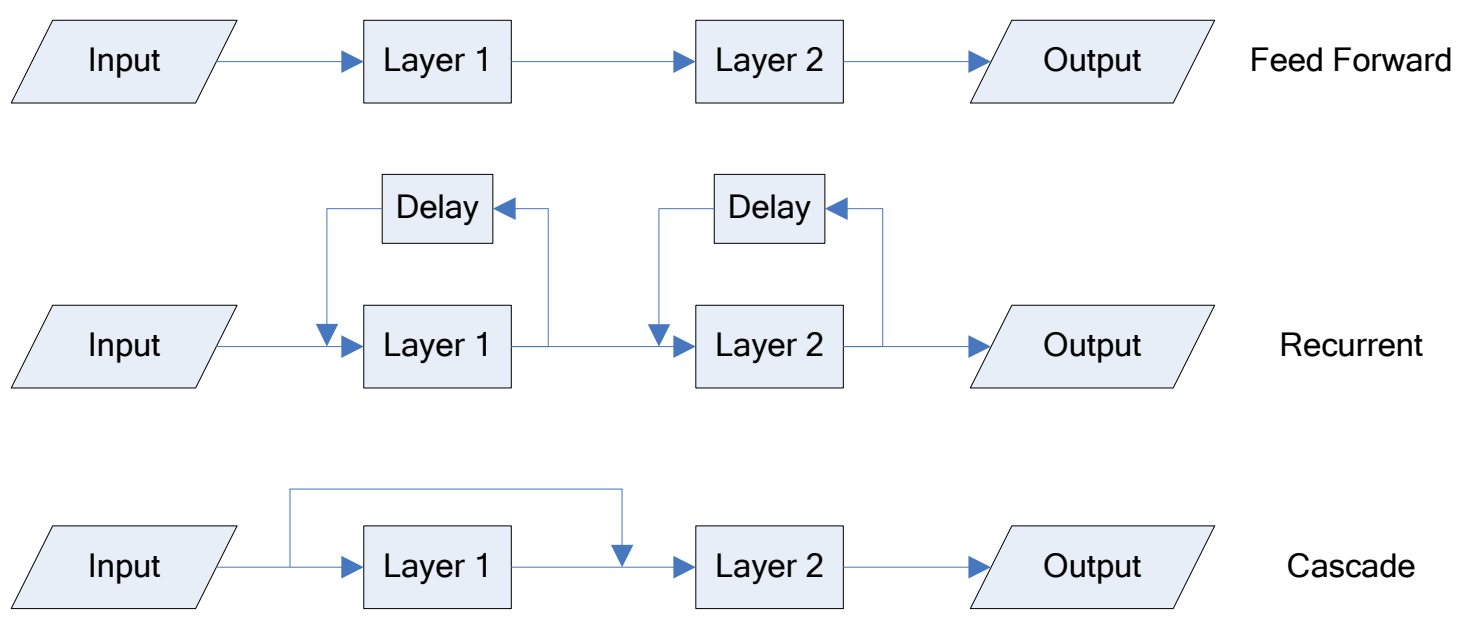

Figure 3-5 Types of Network Layer Interconnections

Figure 3-6, Figure 3-7, and Figure 3-8 show the details of the connections between the neurons of the different layers for each of the types of networks used. Note that the circles containing a "B" represent the bias weights for each neuron. 


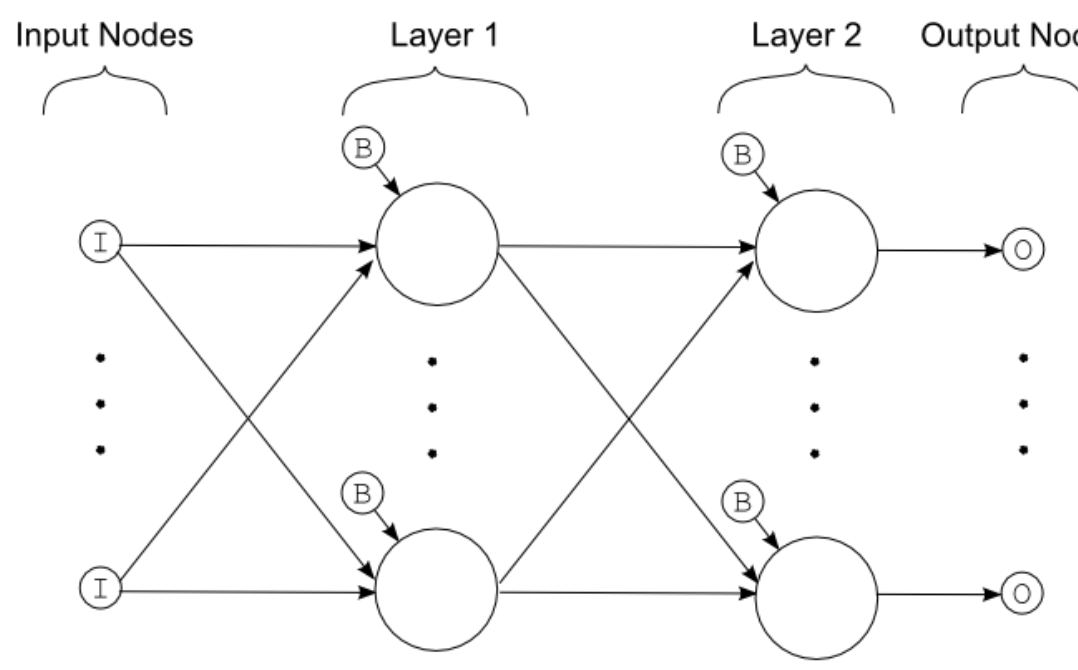

Figure 3-6 Example Neuron Connections for Feed Forward Networks

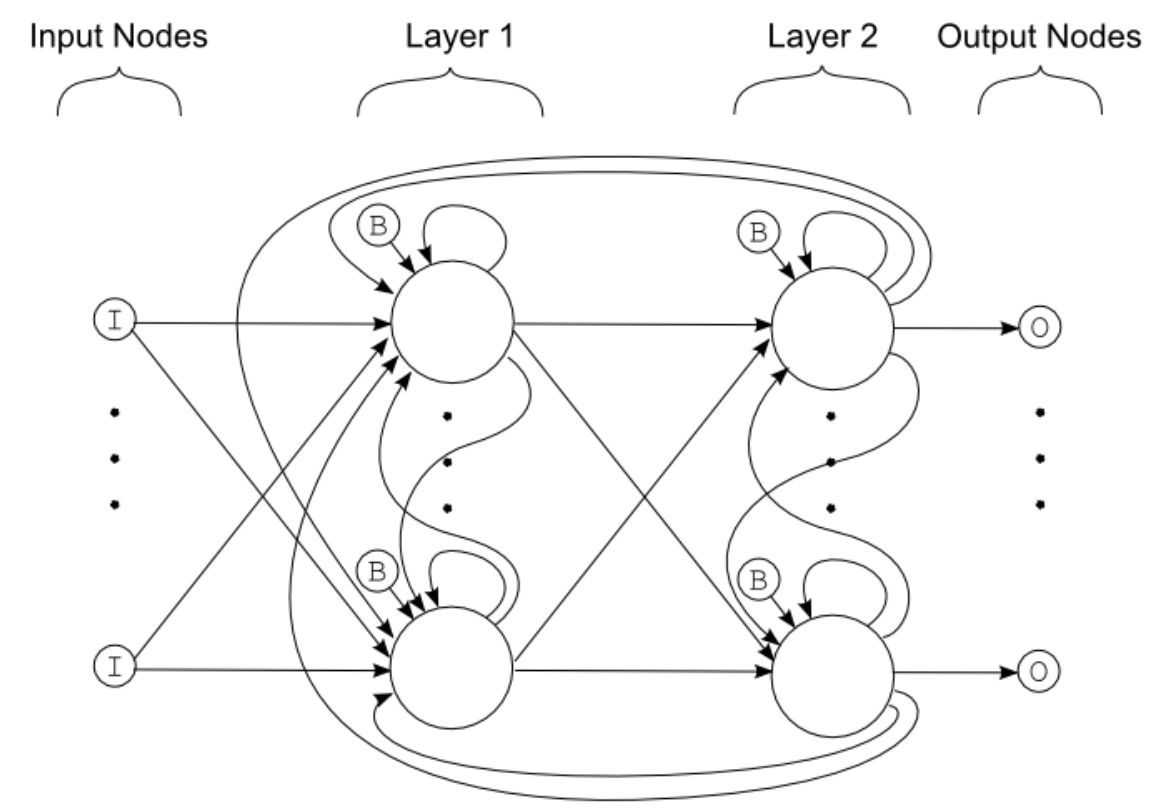

Figure 3-7 Example Neuron Connections for Recurrent Networks 


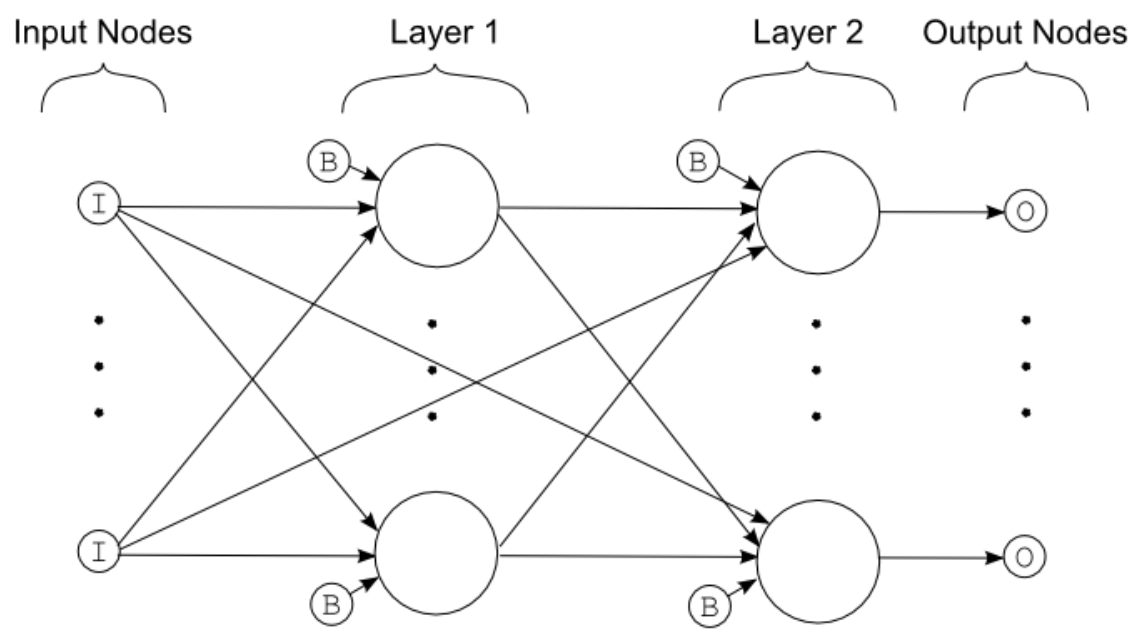

Figure 3-8 Example Neuron Connections for Cascade Networks

\subsubsection{Network Output}

Each neuron in the output layer of the networks represents one of the symbols of the code set. The neuron with the largest output is determined to be the correctly decoded symbol for a given input. When the sampled data points of a single received symbol are not presented to the network at a single time instance, then post processing of the outputs must be performed to arrive at a single decision for a symbol.

The network of Figure 3-3 is a simple example of this. If the code set used for the example contained two symbols then the network would have two output neurons. Each of the neurons would output a distinct value for each of the inputs it was presented. This means that there would be two output values for each neuron. Post processing is required to combine the two output values of each neuron into a single metric that can then be used to determine which neuron has the highest value. 


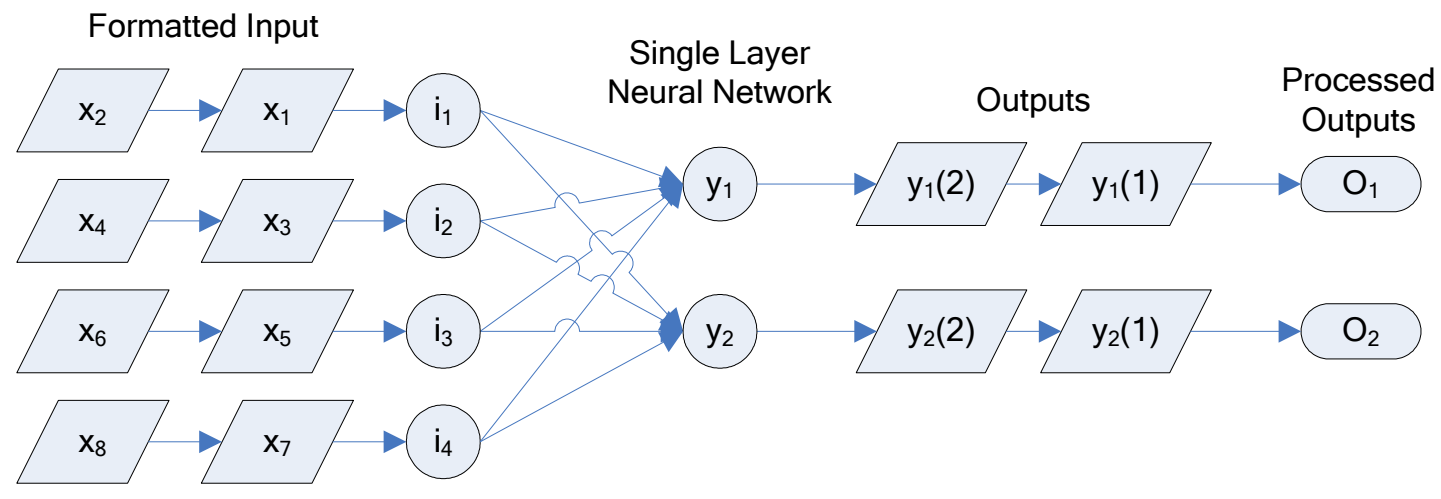

Figure 3-9 Neural Network Inputs and Outputs

The post processing method used is a simple weighted sum of the outputs. Equation 3-12 shows how the multiple outputs, $y_{N}(1)$ and $y_{N}(2)$ for each neuron $N$ of Figure 3-9, are weighted and summed to arrive at a single metric, $O_{N}$.

$$
\begin{gathered}
y_{1}(2) w_{2}+y_{1}(1) w_{1}=O_{1} \\
y_{2}(2) w_{2}+y_{2}(1) w_{1}=O_{2}
\end{gathered}
$$

When each of the weights is identical then the post processing is simply an averaging, or sum, of the individual outputs. Another weighting scheme used in this thesis has the weight assigned to each output increasing over the time it takes for a complete symbol to be presented to the network.

\subsubsection{Similarities with Correlation Receivers}

The simplest neural network receiver evaluated in this thesis is virtually identical to the architecture of a correlation receiver. A neural network that uses linear transfer functions, 
consisting of a single layer, and having an equal number of inputs as samples per symbol, is essentially a bank of linear filters, which is the same architecture as a correlation receiver. In this application, they both have the exact same inputs, same outputs, and same number of filter weights. The difference between the two is the values of the weights and how they are determined. The weights of the neural network are trained whereas the correlation receivers' weights are set to be equal to stored prototypes of each symbol in the symbol set. In digital communication systems, the correlation receiver is the decision stage that follows any preprocessing, such as anti-aliasing filters.

The stored prototypes used in the correlation receiver are ideally modified to account for the transmission channel being used. This makes the correlation receiver identical in function to that of a bank of matched filters, which is optimal in the presence of AWGN [5]. What about situations when the noise is band-limited AWGN, or contains distinct spectral peaks? If the statistical characteristics of the noise are known a priori then it is still possible to use a bank of matched filters, which means a correlation receiver can still be optimum. It is expected that the simple linear neural network will adapt its weights towards that of the optimal correlation receiver. When the statistics of the noise is not known a priori then a linear neural network may be able to perform better than a correlation receiver. The more complex neural network architectures are expected to behave similarly and possibly even better due to the added non-linear processing capability. 


\subsection{Simulation Methods}

Simulations of the EM MWD digital communication system and the neural network receivers were performed with the Matlab Neural Network Toolbox. Scripts and functions were written to test the ability of a static neural network to recognize transmitted symbols in the midst of a wide range of signal to noise ratios for different types of noise.

\subsubsection{AWGN Generation}

The signal to noise ratio definition used in this thesis is the ratio of signal power to noise power.

$$
S N R=\frac{P_{\text {signal }}}{P_{\text {noise }}}=10^{\left(\frac{S N R_{d b}}{10}\right)}
$$

The first step in generating AWGN for a specific SNR is to calculate the average power of the signal. With $N$ equal to the number of samples in the signal $s(k)$, the average power of the signal is given by equation 3-14.

$$
P_{s}=\frac{1}{N} \sum_{k=1}^{N} s^{2}(k)
$$


The next step is to generate an initial random noise signal $n^{\prime}(k)$ of length $N$ with a normal distribution. The function randn is used to generate such a signal with Matlab. The average power of the noisy signal is then calculated.

$$
\begin{aligned}
& n^{\prime}(k)=\operatorname{rand} n(1, N) \\
& P_{n}^{\prime}=\frac{1}{N} \sum_{k=1}^{N}\left(n^{\prime}(k)\right)^{2}
\end{aligned}
$$

The desired average noise power $P_{n}$ is determined by combining equations 3-13 and 3-14.

$$
P_{n}=\frac{P_{s}}{S N R}
$$

The following equations show the derivation of the required noise-scaling factor that results in the desired average noise power.

$$
\begin{gathered}
P_{n}=\alpha P_{n}^{\prime} \\
P_{n}=\frac{\alpha}{N} \sum_{k=1}^{N}\left(n^{\prime}(k)\right)^{2} \\
P_{n}=\frac{1}{N} \sum_{k=1}^{N}\left((\sqrt{\alpha}) n^{\prime}(k)\right)^{2} \\
n(k)=(\sqrt{\alpha}) n^{\prime}(k)
\end{gathered}
$$

\subsubsection{Colored Noise Generation}

White noise has a power spectral density that contains an equal amount of energy in every frequency band, for a given bandwidth. This means that there is as much energy in the 
$0 \mathrm{~Hz}$ to $100 \mathrm{~Hz}$ band as in the $1000 \mathrm{~Hz}$ to $1100 \mathrm{~Hz}$ band for white noise. The PSD of pink noise has an equal amount of energy contained in every octave. It has just as much energy in the $50 \mathrm{~Hz}$ to $100 \mathrm{~Hz}$ band as in the $1 \mathrm{kHz}$ to $2 \mathrm{kHz}$ band. The PSD of pink noise is equal to $1 / \mathrm{f}$, where $\mathrm{f}$ is the frequency. Equation 3-22 shows the method used to generate the PSD for the non-white noise used during simulations. This method allows for generation of noise containing spectral peaks or plateaus with the power spectral density decreasing at various rates as the frequency moves farther away from the peak or plateau. $B W$ is the bandwidth of the plateau, $F_{C}$ is the center frequency of the plateau or peak, and $\beta$ determines the rate at which the PSD decreases as the frequency deviates from the center frequency. Pink noise is generated by setting $\beta$ to $1, B W$ to 0 , and $F_{C}$ to 0 .

$$
P S D(d B)= \begin{cases}\frac{1}{\left|f-F_{C}\right|^{\beta}} & ,\left|f-F_{C}\right|>\frac{B W}{2} \\ \frac{1}{\left(\frac{B W}{2}\right)^{\beta}} & ,\left|f-F_{C}\right| \leq \frac{B W}{2}\end{cases}
$$

\subsubsection{Sampled Noise Data}

Noise sampled at various rig sites was used to corrupt simulated transmission signals for training and evaluating the neural network receivers. The data acquired was separated into sections; one for training, and one for testing. This ensures that the inputs to the networks during testing are not identical to the inputs that the networks were trained on. Dividing the noise sources in this manner helps test each network's ability to generalize. The noise data is scaled as described in section 3.3.1 before being added to the transmission signals. Some tests 
were also performed where the noise used for testing was taken from the same section used for training. The point in doing so is to observe the performance of the networks in an ideal case.

\subsubsection{EM MWD Digital Communication Simulation}

The first step in simulating an EM MWD digital communication system is generating a transmission signal from a known symbol set using a given pulse width and sampling frequency. In order to mimic typical EM MWD communication characteristics, a $200 \mathrm{~Hz}$ sampling frequency ( $5 \mathrm{~ms}$ sampling period) and a $50 \mathrm{~ms}$ pulse width is used. Using these values, with the each symbol in the set shown in equation 3-5, results in 40 samples per symbol. The resulting sampled symbol set is shown in Figure 3-10 

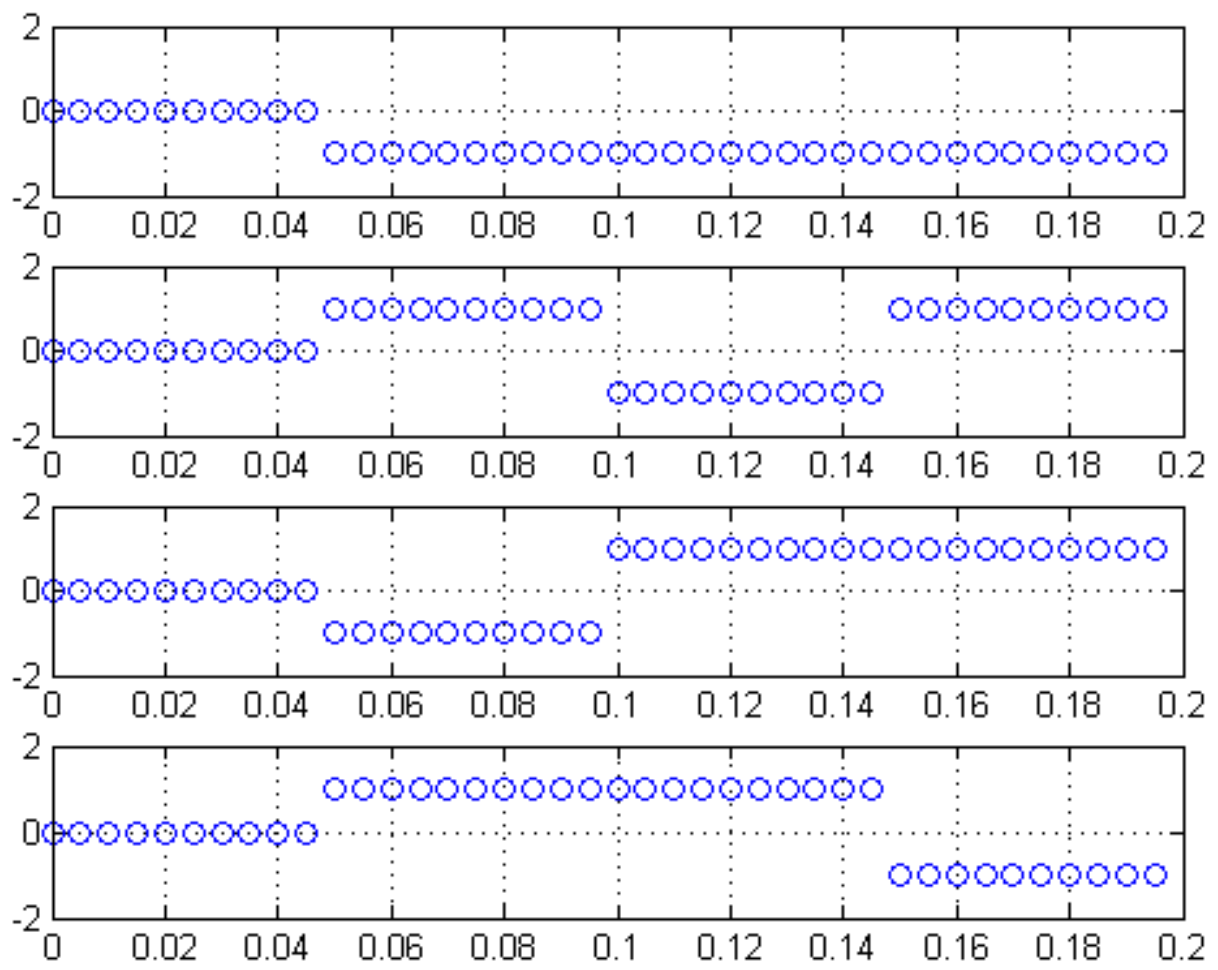

Figure 3-10 Bipolar 4-Ary Orthogonal Symbol Set Sampled at 200Hz with 50ms pulse width

Combining multiple random permutations of the symbol set results in a transmission signal that contains equal occurrences of each symbol. 

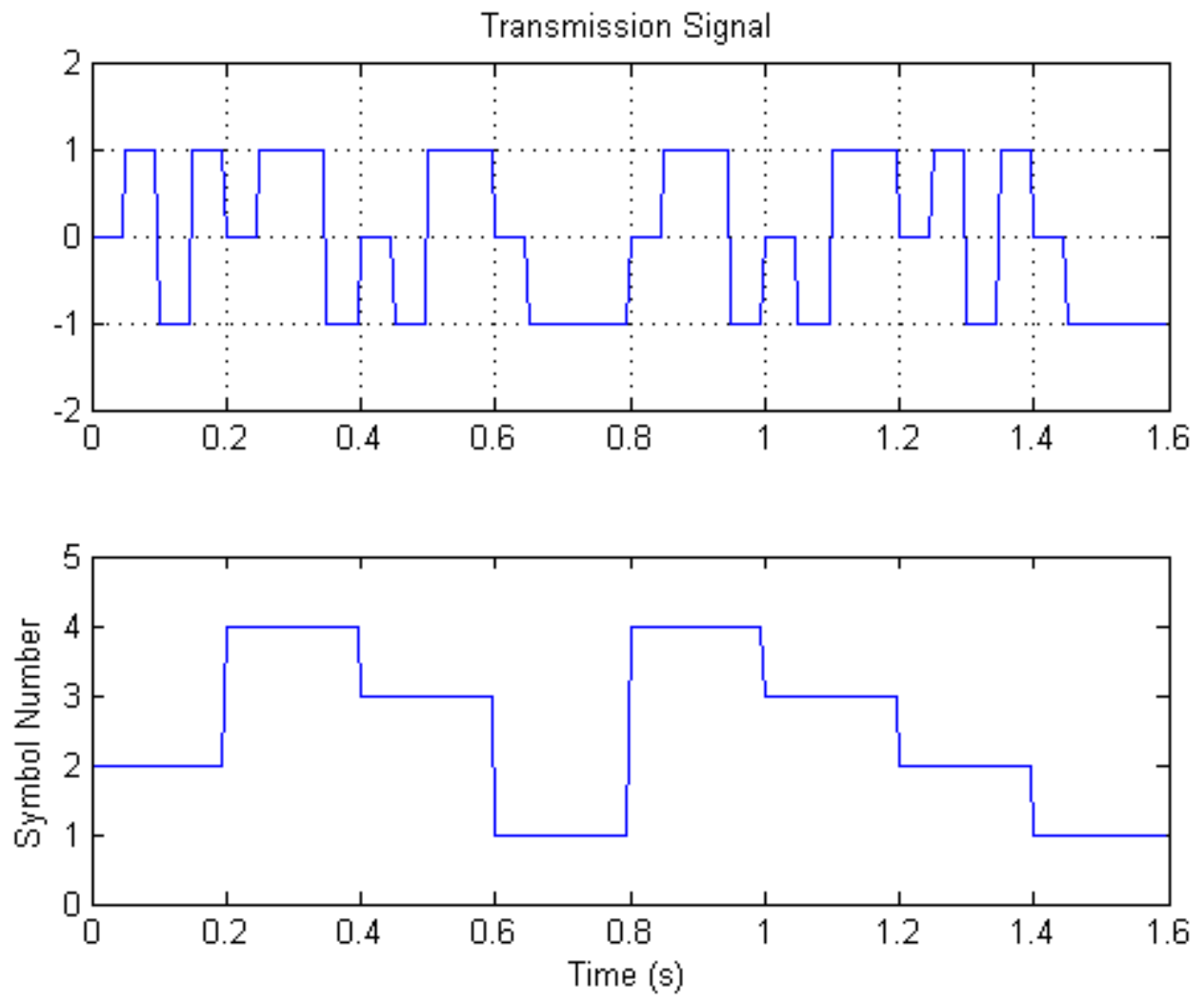

Figure 3-11 Transmission Signal consisting of 2 random permutations of the symbol set

A multiple pole low pass filter chain is used to mimic the filtering that occurs as an EM signal passes through the earth. The simulated transmission signal is passed through this filter chain before noise is added.

The noise used for corrupting the transmission signal is either generated or taken from real world samples. Varying amounts of signal to noise ratios are generated by scaling the corrupting noise as detailed in sections 3.3.1. The noise added to the transmission signal represents the noise picked up by the antenna in an EM MWD system.

More filtering is performed after the noise is added, which corresponds to the various analog and digital filters used in a typical receiver. Some of the simulations in this thesis use 
no post-noise filtering whereas others use a second order low pass filter with varying cutoff frequencies and $\mathrm{Q}$ values.

The final step is to pass the resulting signal to both the neural network receiver and the correlation receiver. Figure 3-12 shows an example of a transmitted signal at the different stages of simulation.

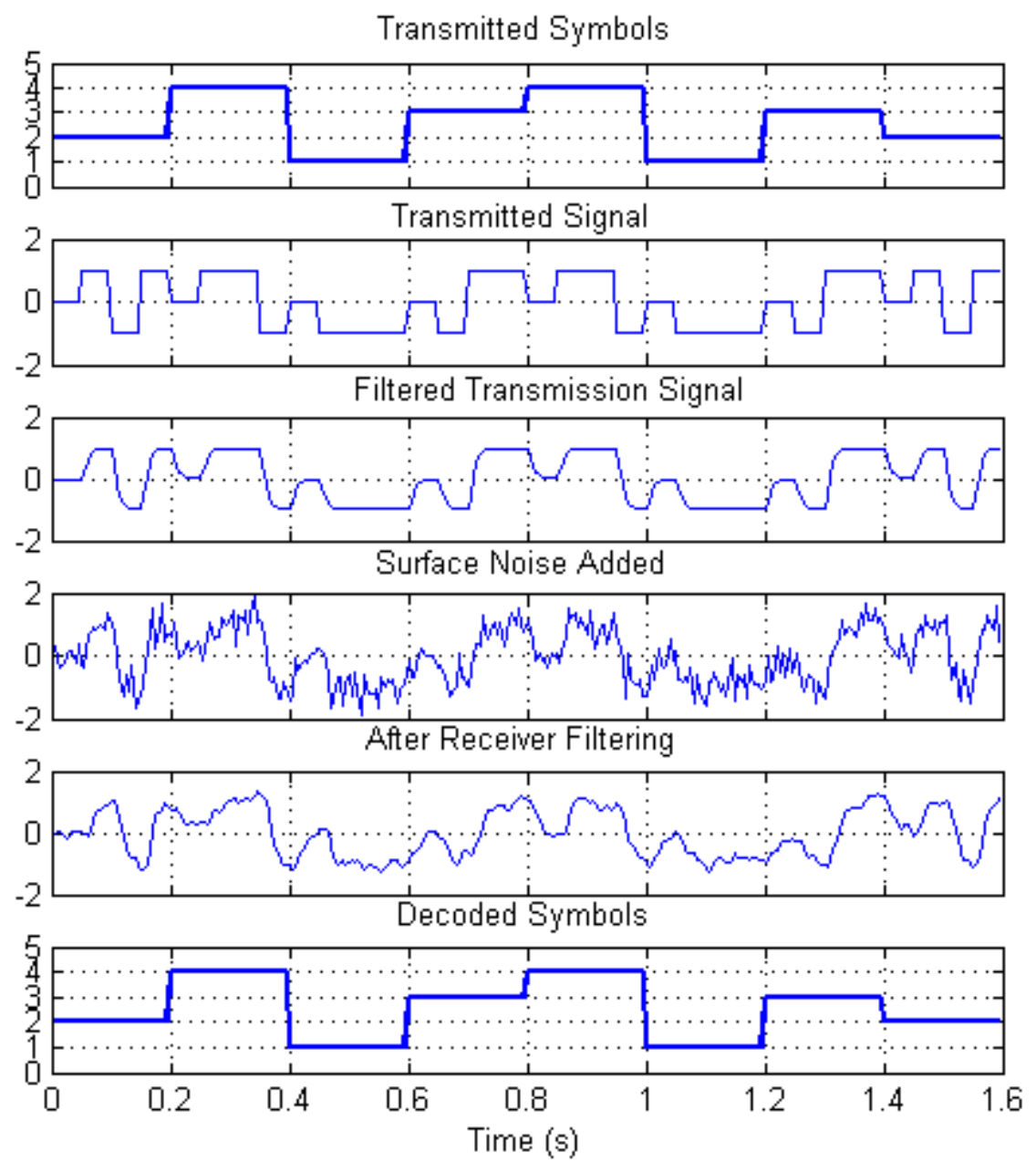

Figure 3-12 EM MWD Simulation Signal Stages 


\subsubsection{Network Training}

The ANN's used in this thesis are trained offline in batch mode. Online training was not attempted. The term batch refers to the method of calculating the error gradient over multiple input vectors, which constitutes a batch, as opposed to online training, which uses a single input vector. Each batch is generated by the method shown in section 3.3.4 and contains multiple sets of symbols. Each set consists of a random permutation of all the symbols of the symbol code set. This results in each symbol in the code set occurring an equal number of times in the training batch. Each set of symbols within the transmitted signal can be corrupted by different signal to noise ratios as well as different filters. There is a constraint on the number of symbol sets present in the training batch in order to give the same amount of training time for each possible combination of SNR and filter type. The number of symbol sets must be a multiple of the number of possible combinations of SNR and filter type. Using multiple SNR's and multiple filters with batch mode allows the gradient to be calculated over a wide operating range. The resulting trained network should perform well over the entire range of SNR's and filters used during training. Multiple ANN's are trained with the exact same inputs and targets during a training session. The training signal is formatted to match the input size of each network. A simple example will now be given using the symbol code set shown in Figure 3-10.

Let us begin with two ANN's, $\boldsymbol{n e t}^{1}$ and $\boldsymbol{n e t}^{2}$, having input sizes of 4 and 8 respectively. We desire to train these networks to function as digital communication receivers in the presence of AWGN with SNR's in the $5 \mathrm{~dB}$ to $15 \mathrm{~dB}$ range. We will train the networks using signals with SNR's of $5 \mathrm{~dB}, 10 \mathrm{~dB}$, and $15 \mathrm{~dB}$ in order to accomplish this. Second order low pass filters will approximate the filtering due to the earth. Two different filters will be 
used during training to represent the range of possible filtering expected. The first filter will have a corner frequency of $80 \mathrm{~Hz}$, and a $\mathrm{Q}$ value of 0.3 . The second filter will have a corner frequency of $10 \mathrm{~Hz}$, and a $\mathrm{Q}$ value of 0.5 . The selection of corner frequencies and $\mathrm{Q}$ values for this example is meant to include the wide possible range of transmission channels encountered from drill site to drill site [22]. The number of symbol sets contained in the batch size must be a multiple of the number of combinations possible by choosing one SNR and one filter. The number of combinations is six in this case. Using six symbol sets in the training batch, with each symbol set containing four symbols, and each symbol containing 40 samples, results in a total of 960 samples. In this case, each symbol set will be modified by a unique combination of SNR and filtering. If 12 symbol sets had been chosen, then two symbol sets would be modified by every unique combination of SNR and filtering. Each unique combination of SNR and filtering will be used to simulate an EM MWD transmission, as described in section 3.3.4. Figure 3-13 shows one possible training signal of minimum length for this setup. 
Transmitted Symbols

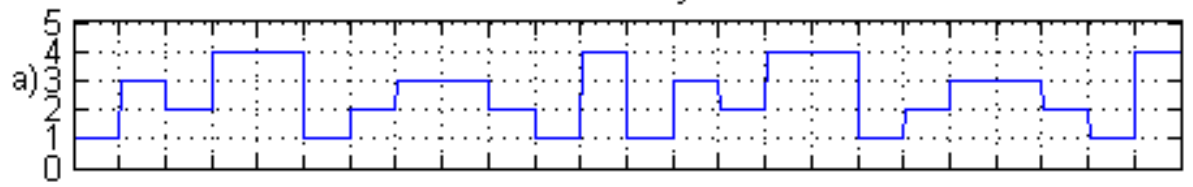

Transmitted Signal

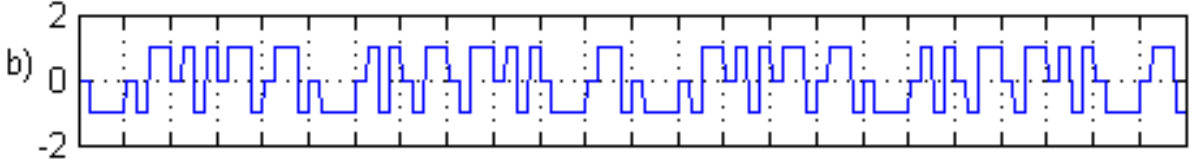

Filtered Signal

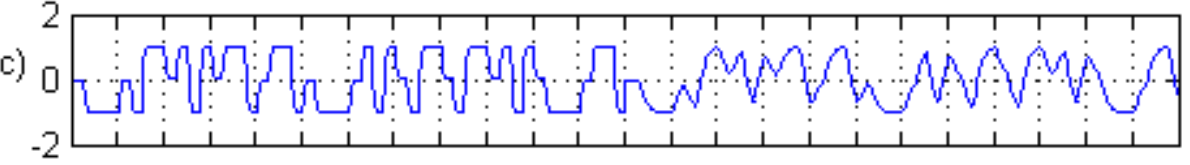

Signal With Added Noise

d)

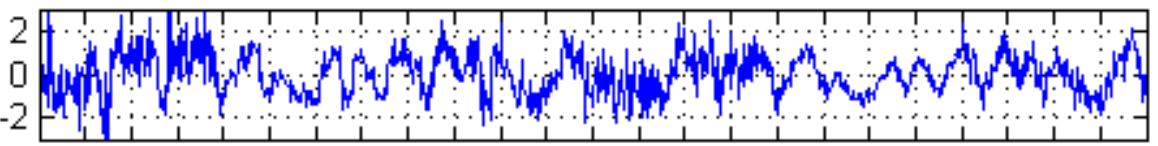

SNR Level in Decibels

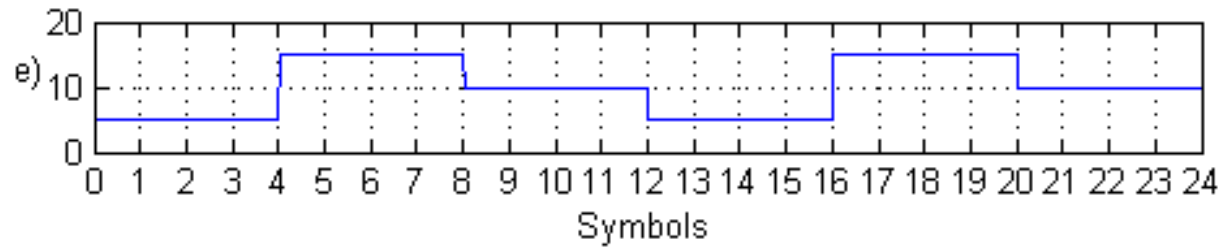

Figure 3-13 Signal Generation for Network Training a) Transmitted symbol id's consisting of 6 random permutations of the symbol set. b) Corresponding Digital Signal. c) $1^{\text {st }}$ half is filtered by $80 \mathrm{~Hz} \mathrm{LPF}, 2^{\text {nd }}$ half is filtered by $10 \mathrm{~Hz}$ LPF. d) 3 different levels of noise added to the filtered signal. e) SNR levels of resulting signals.

Once every portion of the training batch has been simulated, then the resulting corrupted transmission signal is formatted to match the input size of each network. The input to net $^{1}$ must be a sequence of vectors of length 4 , whereas input vectors of size 8 are required for $\boldsymbol{n} \boldsymbol{e} \boldsymbol{t}^{2}$. See 3.2.1 for information on the relationship between input size and number of input vectors per symbol. The training batch will therefore be formed into 240 vectors of length 4 for $\boldsymbol{n e t}^{\mathbf{1}}$, and 120 vectors of length 8 for $\boldsymbol{n e t}^{2}$. Figure 3-14 below shows an example of filtered 
input signal, as it would be formatted for $\boldsymbol{n e t}^{2}$. The figure shows the settling time due to the filtering.

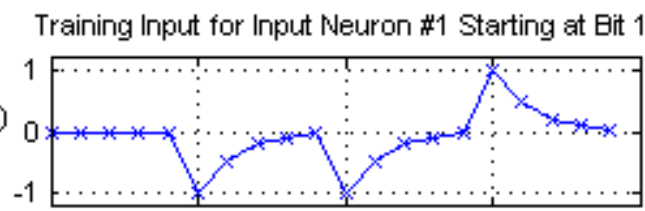

Training Input for Input Neuron \#3 Starting at Bit 2

c)

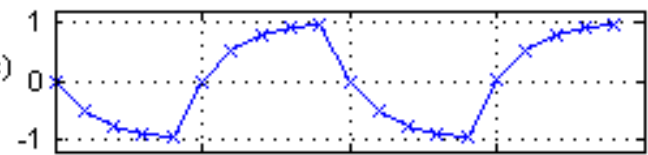

Training Input for Input Neuron \#5 Starting at Bit 3

e)

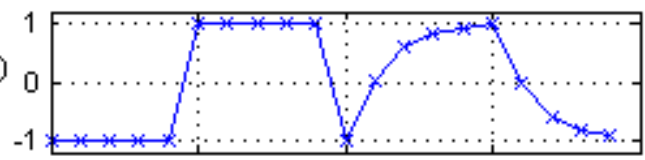

Training Input for Input Neuron \#7 Starting at Bit 4

g)

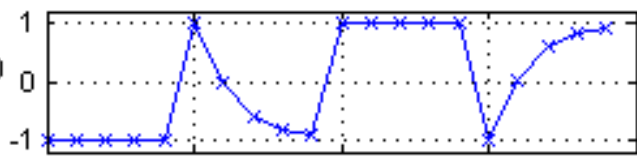

Training Input Signal

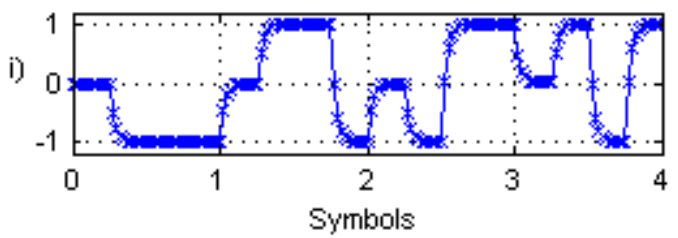

Training Input for Input Neuron \#2 Starting at Bit 1.5

b)

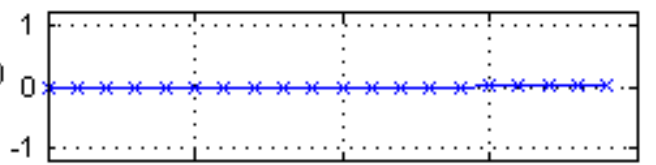

Training Input for Input Neuron \#4 Starting at Bit 2.5

d)

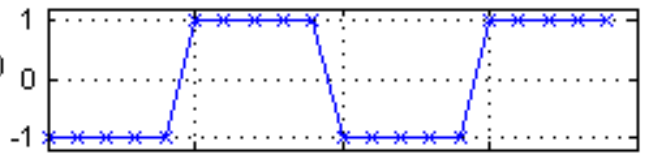

Training Input for Input Neuron \#6 Starting at Bit 3.5

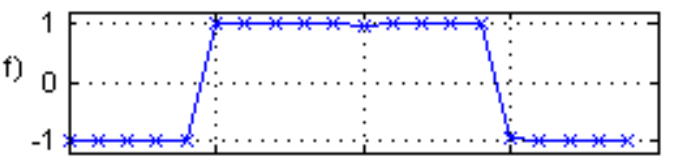

Training Input for Input Neuron \#8 Starting at Bit 4.5

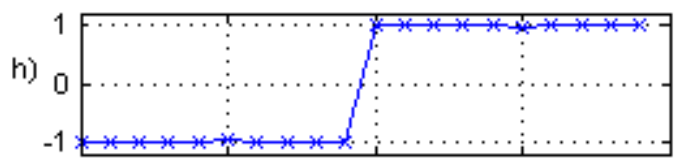

Symbol \#'s of Training Signal

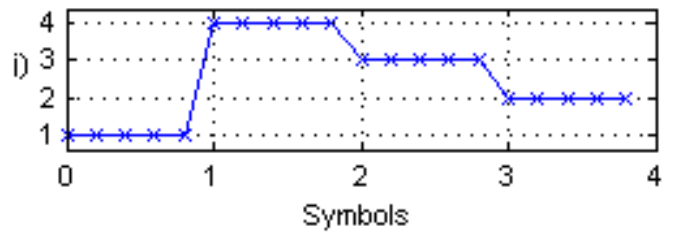

Figure 3-14 Example of input formatting for $n e t^{2}$. The training input signal of plot i is divided up for the 8 input neurons as shown in plots a through $h$. Plot $\mathbf{j}$ shows the symbols used for training.

Each output neuron of a network represents one of the symbols in the code symbol set.

The corresponding output neuron for a transmitted code must be trained to output a 1 when that code is presented to the network. Therefore, when a network is being trained to identify the $1^{\text {st }}$ symbol of a symbol set, than the target value for the $1^{\text {st }}$ output neuron would be a 1 . For 
the $2^{\text {nd }}$ symbol the target value for the $2^{\text {nd }}$ neuron would be set to 1 . The target values for all neurons that do not correspond to the symbol being identified are set to 0 or -1 depending on the output neurons transfer function. Figure 3-15 shows training targets for a typical training batch.

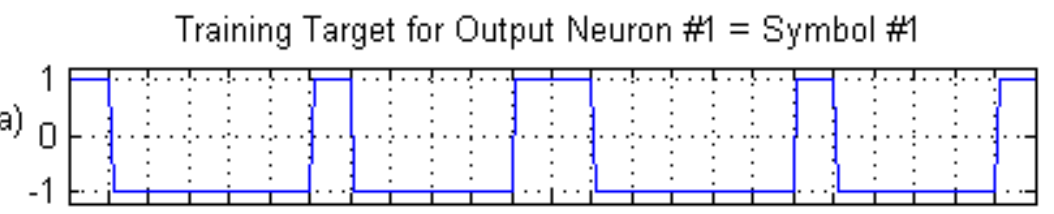

Training Target for Output Neuron \#2 = Symbol \#2

b)

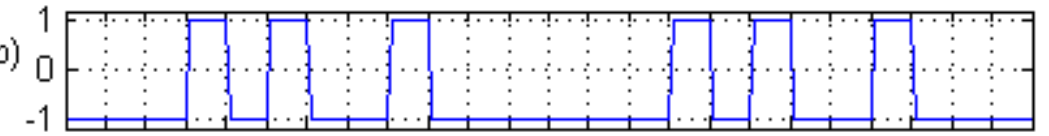

Training Target for Output Neuron \#3= Symbol \#3

c)

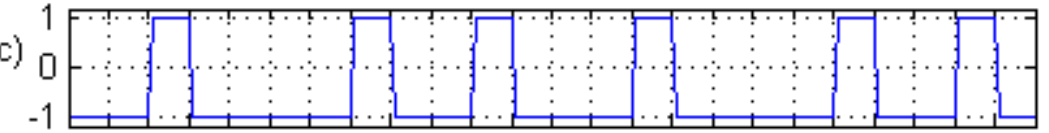

Training Target for Output Neuron \#4= Symbol \#4

d)

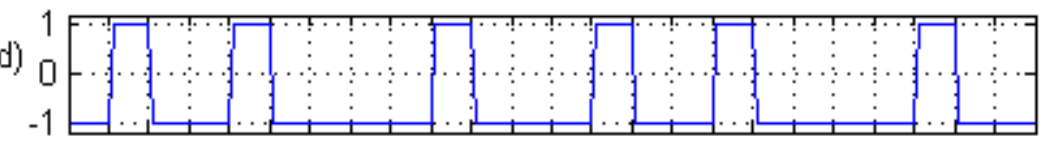

Symbol \#s of Output Training Target

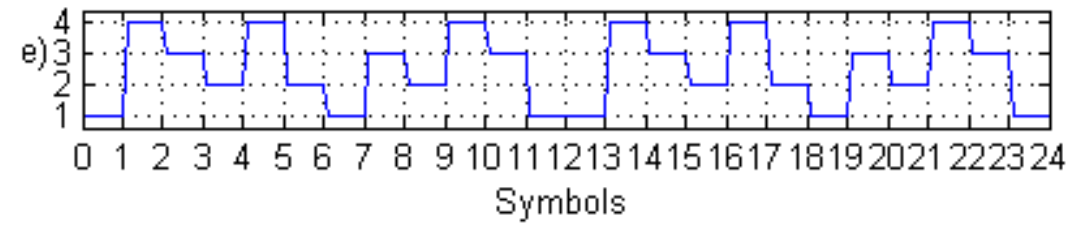

Figure 3-15 Training targets for a symbol set containing 4 symbols

The overall training algorithm is shown in Figure 3-16. 


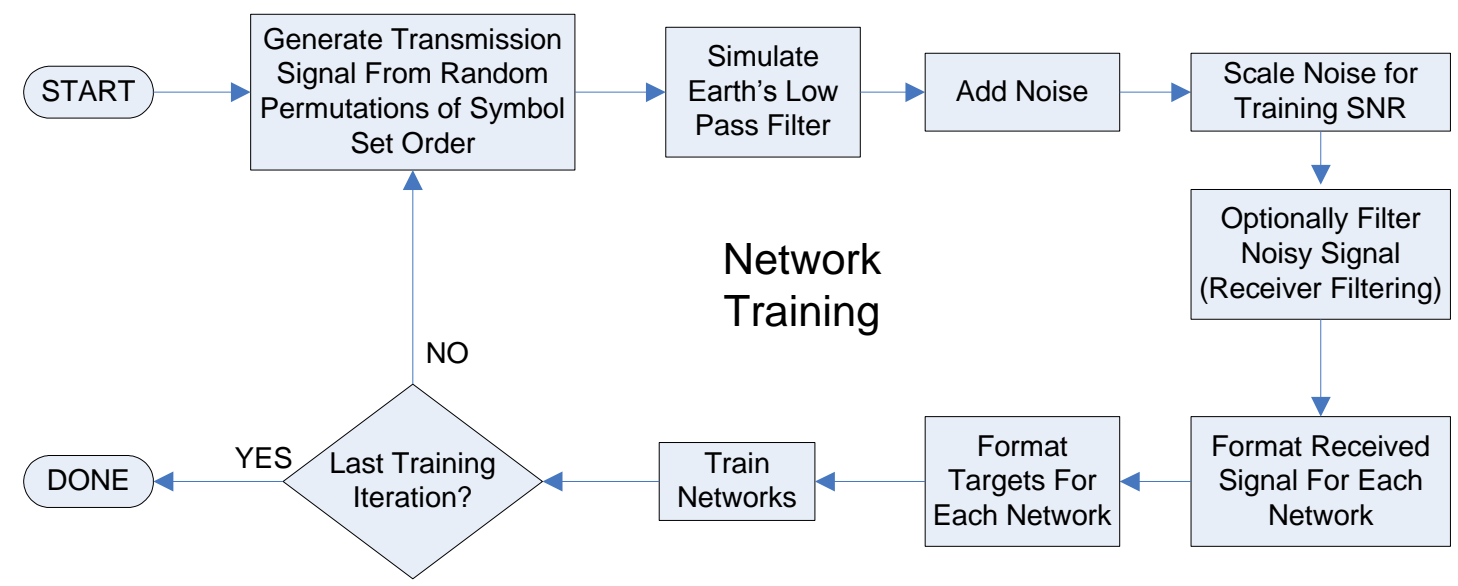

Figure 3-16 Neural Network Receiver Training Flow Chart

\subsubsection{Receiver Testing}

Testing of the simulated digital communication receivers of this thesis involves generating symbol error rate (SER) plots. These plots are used to compare the performance of various types of receivers. Figure 3-17 shows the process flow for testing of the neural network receivers, as well as the correlation receivers. 


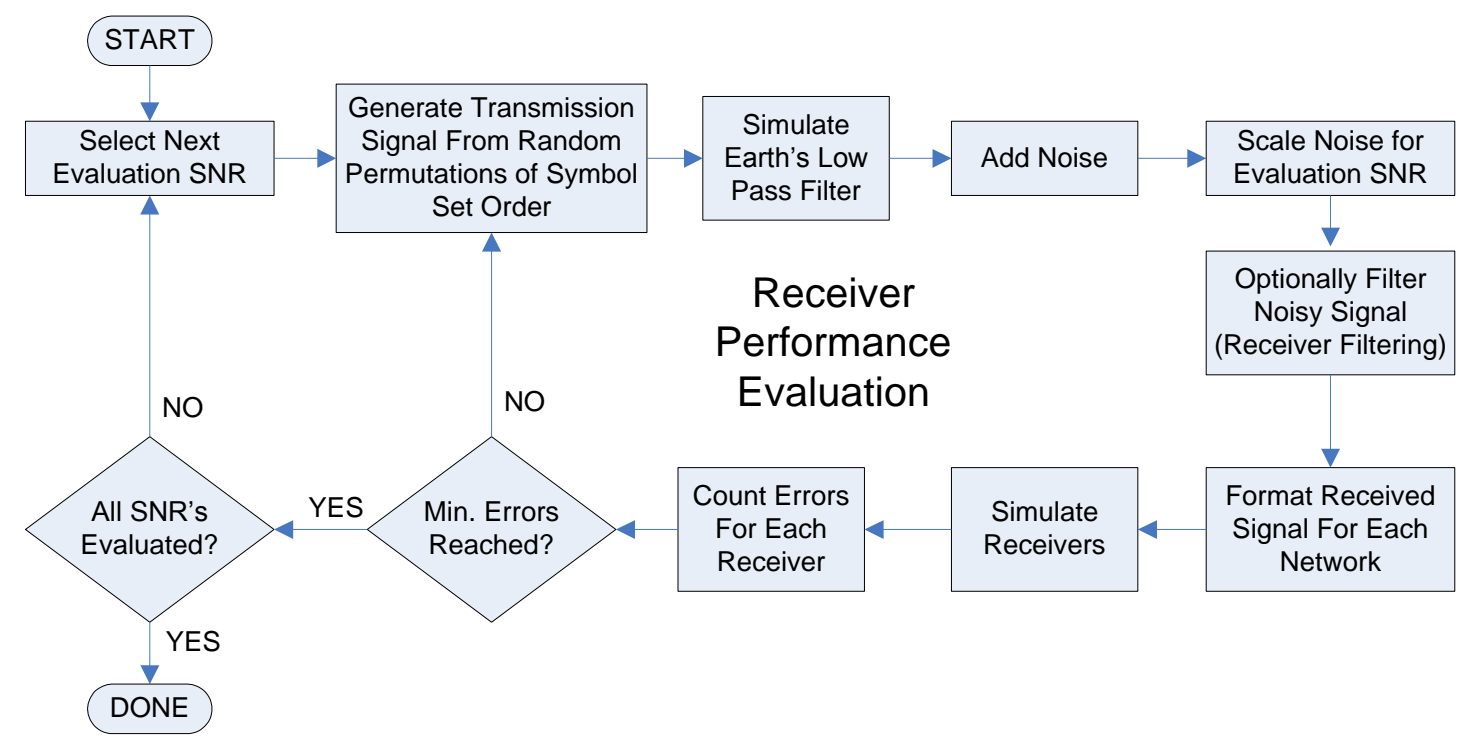

Figure 3-17 EM Digital Communication Receiver Performance Evaluation Flow Chart

The method for generating an input to the receivers for testing is almost identical to what is done for training the neural networks. The main difference is that only one SNR value is used at a time during testing, whereas training involves using multiple values in a single batch. The SNR values used during testing does not always match the values used during training in order to test the various networks' ability to generalize.

In order to obtain tight confidence intervals around the SER, as was discussed in section 2.2.3, it is necessary to simulate a large number of transmitted symbols. As was done in the first phase, random orders of symbols are put together to create a transmission signal. All the networks are then simulated with this signal after it has been filtered and corrupted with noise. A tally of the total number of errors for each receiver is kept. The receivers are repeatedly simulated until a minimum number of errors occur or a maximum number of symbols are transmitted. All of this is repeated with varying signal to noise ratios to obtain the data needed for the SER plots. 


\subsubsection{Correlation Receiver Implementation}

Correlation receivers match a sampled signal with the most likely symbol in the symbol set. In order to do this the correlator must store copies of the symbols contained in the set. The earth and any preprocessing the receiver implements distort the signal that is seen by the correlator. For this thesis, the stored copies of the symbols are ideal in the sense that they are distorted by the exact filters that the transmitted signals pass through. If no noise corrupts the transmitted signals, than the received signal will exactly match one of the stored symbols [5]. In the real world, the transfer function of the earth is unknown and must be estimated. Correlation receiver performance improves as the accuracy of the estimation increases. 


\section{Chapter 4 Simulations and Results}

This chapter contains the results of numerous ANN digital communication receiver simulations. Each simulation contains a few different neural network architectures and/or training parameters that are trained and tested with exactly the same inputs. The variations in architectures include the number of layers, layer sizes, input sizes, transfer functions, as well as the inclusion or exclusion of recurrent connections. Variations in training parameters include number of epochs, batch size, learning rate, and training algorithms. Multiple ANNs were simulated for each variation, each of which was initialized with different connection weights. A single correlation receiver is also included in each simulation for performance comparison.

M-ary bipolar orthogonal signaling was used with k set to 2 and 3, corresponding to 4 and 8 symbols per set respectively. Six different noise sources were used during simulations. The first noise source is AWGN, which is the typical corrupting noise source for measuring the performance of a digital communication receiver. The second type of noise has a non-flat power spectral density. Various PSD shapes were used during simulations. The last four noise sources are actual sampled noise data taken from drilling sites.

\subsection{Presentation Format}

Each test variation will be presented by two figures and two tables. First, a figure depicting the connections between the different layers for each type of network is given. This is followed by a table that details the network architecture as well as the training and test parameters used. The symbol error rate (SER) results from each test are then shown graphically in a modified waterfall plot. 


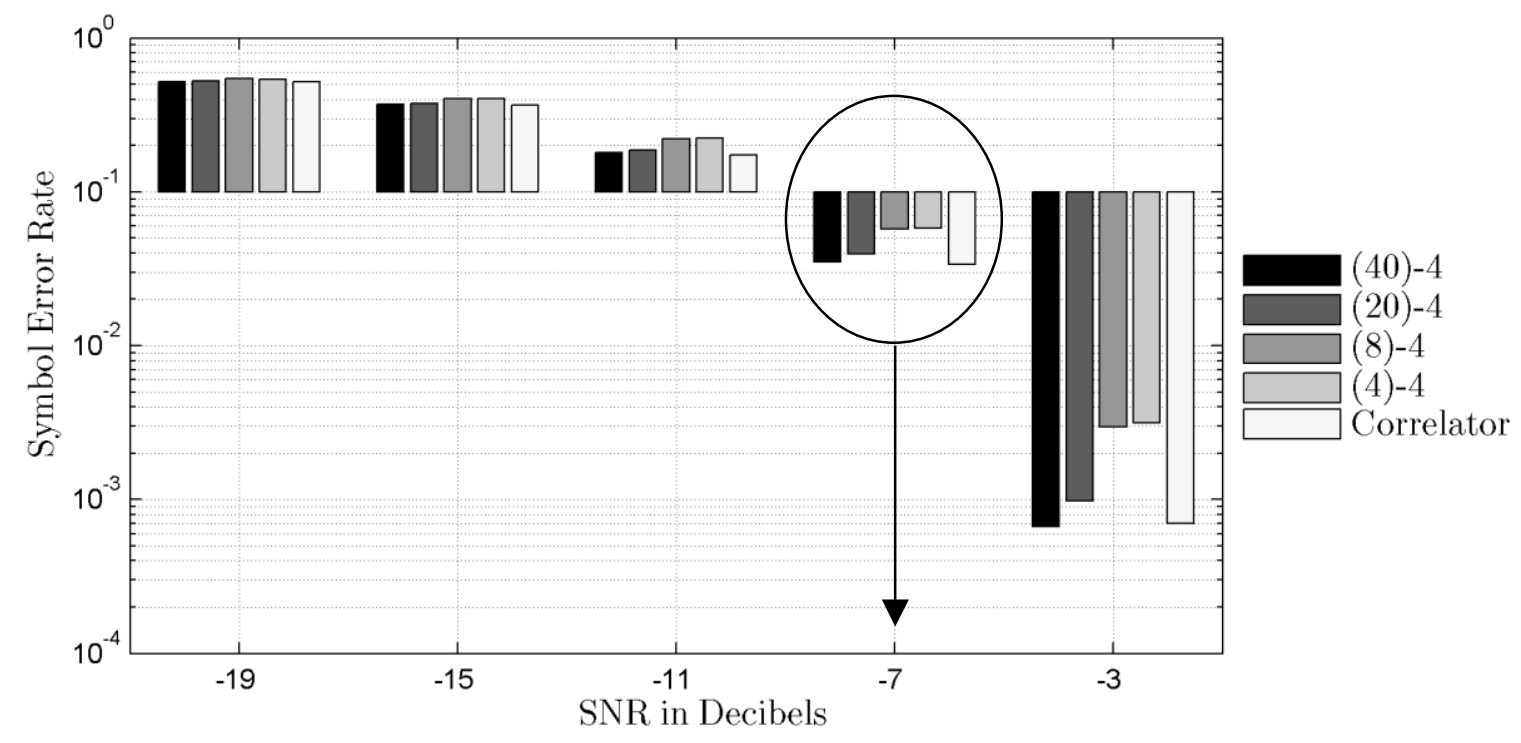

Figure 4-1 Example SER Plot. The circled bars correspond to the SER for the receivers at a SNR of -7dB.

The values used in generating the SER plot are finally tabulated along with the total number of symbols transmitted for each network. An example of the tabulated SER data is shown below.

\begin{tabular}{|ccccrr|}
\hline \multicolumn{7}{c|}{ SNR } \\
\hline Receiver & $\mathbf{- 1 9 d B}$ & $\mathbf{- 1 5 d B}$ & $\mathbf{- 1 1 d B}$ & $\mathbf{- 7 d B}$ & -3dB \\
\hline$(40)-4$ & 0.51719 & 0.37085 & 0.18211 & 0.03498 & 0.00080 \\
$(20)-4$ & 0.51638 & 0.37110 & 0.18383 & 0.03542 & 0.00075 \\
$(8)-4$ & 0.53249 & 0.39596 & 0.21138 & 0.05178 & 0.00268 \\
$(4)-4$ & 0.54432 & 0.41509 & 0.24211 & 0.07648 & 0.00833 \\
correlator & 0.51465 & 0.36817 & 0.17876 & 0.03289 & 0.00068 \\
\hline \# Symbols Tested & 42,600 & 42,600 & 42,600 & 42,600 & 42,600 \\
\hline
\end{tabular}

Table 4-1 Example SER Data Table

Each of the receivers tested are shown in the first column. Each row contains the symbol error rate (SER) for the receiver at different SNR levels, which are shown in the column header. The last row shows the total number of symbols transmitted for each SNR level during the 
testing of the receivers. The SER is the ratio of symbol errors to total symbols transmitted, which should not be confused with bit error rate (BER). A SER of 0.1 indicates that 10 percent of the symbols used during testing were incorrectly decoded.

\subsection{Notes on Simulations and Results}

A training epoch is the term used to denote a single training iteration. During each epoch, every symbol in the training batch is presented to the network and the error for each output neuron is accumulated. After all symbols have been presented, the error is used to determine the change to the weights and biases of the network according to the training function. See section 2.3.7 for more information about backpropagation.

The size of the training batch is a multiple of the number of symbols in the codeset multiplied by the number of training SNR levels. Typically, there will be 200 or 300 occurrences of each symbol for every training SNR level, with the symbol order randomly set. For example, a training epoch for networks using a codeset of 4 symbols and 3 training SNRs would result in a batch size of 3,600 if each combination of symbol and SNR was repeated 300 times.

Multiple training epochs make up a training session. In this thesis, the number of epochs is normally set to 100. Each of the epochs in a training session uses the exact same training batch. Multiple training sessions are used to provide more examples for each network to train with while not requiring excessively large batch sizes. The use of multiple training sessions should also help the networks' ability to generalize. The number of training sessions is usually 
set to 2. Using these numbers results in each network having its weights updated 200 times before the network is tested to determine its SER.

The performance metric used in this thesis is the symbol error rate (SER) and not the bit error rate (BER). Each symbol transmitted results in either a successful reception or a failure. The calculation of the SER is simply the number of symbols that were not correctly identified by the receiver, divided by the total number of symbols sent. The format of the SER plots used in this thesis is not the same as the standard waterfall plots typically used in digital communications. The reason for deviating from this standard is to make it easier to visually distinguish the SER of different networks plotted on the same plot. Notice that the SER plots contain bars that stem from the point 0.1 , and reach down or up to the SER for each network tested. The reason for this is that a SER of 0.1 in EM MWD communication is an estimate of the upper limit that would allow a drilling operation to successfully continue. The point at which the bars change from extending upwards to extending downwards marks the minimum operable SNR for that network.

Multiple networks are simulated for each of the neural network receiver architectures. Each of the networks of a given type is initialized with different weights and bias values. The average performance of the networks is used as an indication of the performance for that specific type of network architecture. The reason for having multiple networks is to show that the networks can consistently converge to a good solution from different starting points.

The exact definitions of all the neuron transfer functions used are given in section 3.2.2. They will be referred to as SIG, RB, LIN, and SLINS. 


\subsection{Default Parameters for Training Functions}

There are three different training functions used for the networks in this thesis. To simplify the graphs and tables they will be given shorter names. The function GDX implements a gradient descent with momentum and adaptive learning rate.

$$
\begin{gathered}
\Delta w_{k}=m_{c} \cdot \Delta w_{k-1}+\alpha \cdot m_{c} \cdot g_{k} \\
w_{k}=w_{k}+\Delta w_{k}
\end{gathered}
$$

This training function is used in the majority of the simulations. The learning rate, $\alpha$, and momentum constant, $m_{c}$, will be specified for each network that uses GDX. The learning rate is increased after every weight adaptation that results in a performance increased.

Likewise, whenever the performance decreases due to a change in weights, the learning rate is decreased and the change to the weights is undone. The default parameters that are used for GDX unless otherwise specified are shown in the following table.

\begin{tabular}{|c|c|}
\hline Parameter & VALUE \\
\hline Ratio to increase learning rate & 1.05 \\
\hline Ratio to decrease learning rate & 0.7 \\
\hline $\begin{array}{c}\text { Maximum performance increase allowed } \\
\text { before learning rate is decreased }\end{array}$ & 1.04 \\
\hline Momentum Constant & 0.7 \\
\hline
\end{tabular}

Table 4-2 Default Parameters for Training Function GDX 
The function CGP implements a conjugate gradient backpropagation with PolakRibiere updates. With all conjugate gradient methods, the direction in which the weights will be updated, $\Delta w_{k}$, is a combination of the current gradient, $g_{k}$, and the previous update direction, $\Delta w_{k-1}$.

$$
\begin{gathered}
\Delta w_{k}=-g_{k}+\Delta w_{k-1} Z \\
w_{k+1}=w_{k}+\alpha \cdot \Delta w_{k}
\end{gathered}
$$

The parameter $Z$ is updated according to the Polak-Ribiere variation [12].

$$
Z=\frac{\left(\left(g_{k}-g_{k-1}\right)^{\prime} g_{k}\right)}{\left\|g_{k-1}\right\|}
$$

The reason for using the Polak-Ribiere variation is that it performed the best during initial comparisons between a few conjugate gradient methods. The parameter that affects the performance of CGP is the line search function used to determine the step size, $\alpha$, to take along the search direction, $\partial w_{k}$. The line search used is based on Charalambous' method, which is a hybrid search using cubic interpolation and a type of sectioning. The step size is set by the line search method so that it results in reaching a minimum along the search direction.

The last training function used is backpropagation utilizing the Levenberg-Marquardt algorithm (LM). The LM algorithm interpolates between the Gauss-Newton algorithm and the gradient descent method. A dampening factor, $\mu$, is varied to cause the algorithm to act more like gradient descent when further away from the minimum and like Gauss-Newton as it approaches the minimum. The parameter $\mu$ is increased whenever the change in weights of the network results in a performance increase. Likewise, it is decreased whenever the 
performance decreases. The algorithm uses an approximation to the Hessian as is shown below. Note that $J$ is the Jacobian in the following equations.

$$
\begin{gathered}
H=J^{T} J \\
w_{k+1}=w_{k}-\frac{g_{k}}{H+\mu I}
\end{gathered}
$$

The default parameters for the LM algorithm are shown below.

\begin{tabular}{|c|c|}
\hline Parameter & VALUE \\
\hline Ratio to increase $\mu$ & 10 \\
\hline Ratio to decrease $\mu$ & 0.1 \\
\hline Initial $\mu$ & 0.001 \\
\hline
\end{tabular}

Table 4-3 Default Parameters for Training Function LM

\subsection{Simulations Using AWGN and 4 Symbols}

\subsubsection{Single Layer Linear Network Simulations}

Each neural network simulated in this section contains a single layer. The number of neurons contained in the layer is equal to the number of symbols. The output of each neuron is an output of the network.

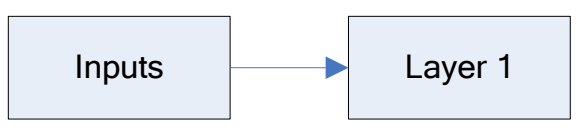

Figure 4-2 Layer Connections for Neural Networks of Figure 4-3 


\begin{tabular}{|c|c|c|c|c|}
\hline Parameter & Value & Value & Value & Value \\
\hline Network Identifier & $(40)-4$ & $(20)-4$ & $(8)-4$ & $(4)-4$ \\
\hline Number if Inputs & 40 & 20 & 8 & 4 \\
\hline Layer 1 Neurons & 4 & 4 & 4 & LIN \\
\hline Layer 1 Transfer Function & $L I N$ & $L I N$ & $G D X$ & GDX \\
\hline Training Function & $G D X$ & $G D X$ & 0.05 & 0.05 \\
\hline Initial Learning Rate & 0.05 & 0.05 & 3 & 3 \\
\hline Number of Networks & 3 & 3 & 3,600 Symbols & 3,600 Symbols \\
\hline Training Batch Size & 3,600 Symbols & 3,600 Symbols & $-11,-7,-3 \mathrm{~dB}$ & $-11,-7,-3 \mathrm{~dB}$ \\
\hline Training SNR(s) & $-11,-7,-3 \mathrm{~dB}$ & $-11,-7,-3 \mathrm{~dB}$ & 100 & 100 \\
\hline $\begin{array}{c}\text { Training Epochs per } \\
\text { Training Session }\end{array}$ & 100 & 100 & 2 & 2 \\
\hline \# of Training Sessions & 2 & 2 & & \\
\hline
\end{tabular}

Table 4-4 Training and Test Parameters for Figure 4-3

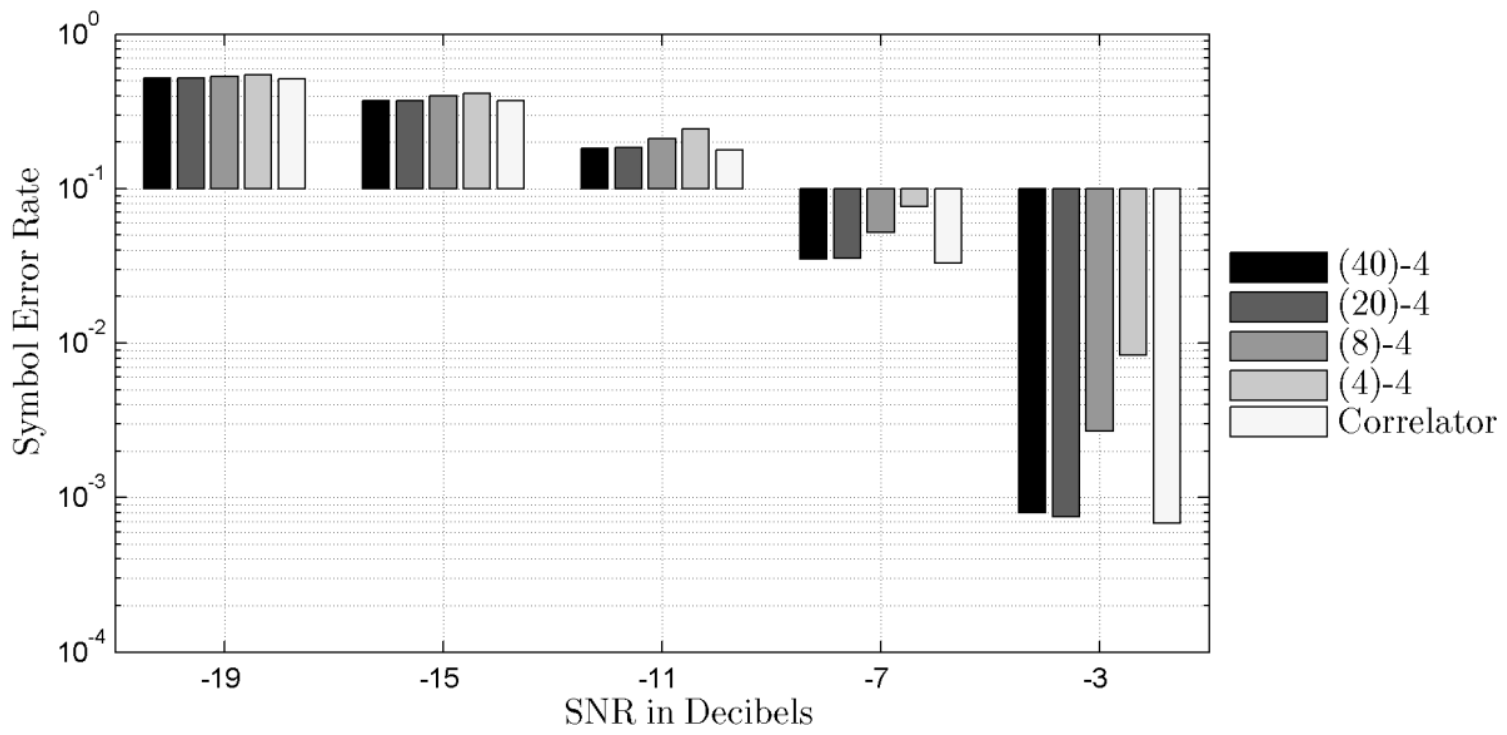

Figure 4-3 Single Layer Linear Network Simulation Results for AWGN with 4 Symbols 


\section{SNR}

\begin{tabular}{|ccrrrr|}
\hline Receiver & $\mathbf{- 1 9 d B}$ & $\mathbf{- 1 5 d B}$ & \multicolumn{1}{c|}{$\mathbf{1 1 d B}$} & \multicolumn{1}{c|}{$\mathbf{d d B}$} & -3dB \\
\hline$(40)-4$ & 0.51719 & 0.37085 & 0.18211 & 0.03498 & 0.00080 \\
$(20)-4$ & 0.51638 & 0.37110 & 0.18383 & 0.03542 & 0.00075 \\
$(8)-4$ & 0.53249 & 0.39596 & 0.21138 & 0.05178 & 0.00268 \\
$(4)-4$ & 0.54432 & 0.41509 & 0.24211 & 0.07648 & 0.00833 \\
correlator & 0.51465 & 0.36817 & 0.17876 & 0.03289 & 0.00068 \\
\hline \# Symbols Tested & 42,600 & 42,600 & 42,600 & 42,600 & 42,600 \\
\hline
\end{tabular}

Table 4-5 SER Data for Figure 4-3

It is clear that the correlation receiver outperforms all the simulated networks.

However, as the input size increases, the performances of the networks approach that of the correlation receiver. Figure 4-4 shows the connection weights of the first trained network containing 40 inputs. Notice how the connection weights of the trained network relate to the ideal reference signals used by the correlation receiver. The network weights have gravitated towards the best possible configuration of weights for the AWGN noise source, which is known to be a correlation receiver [5]. 


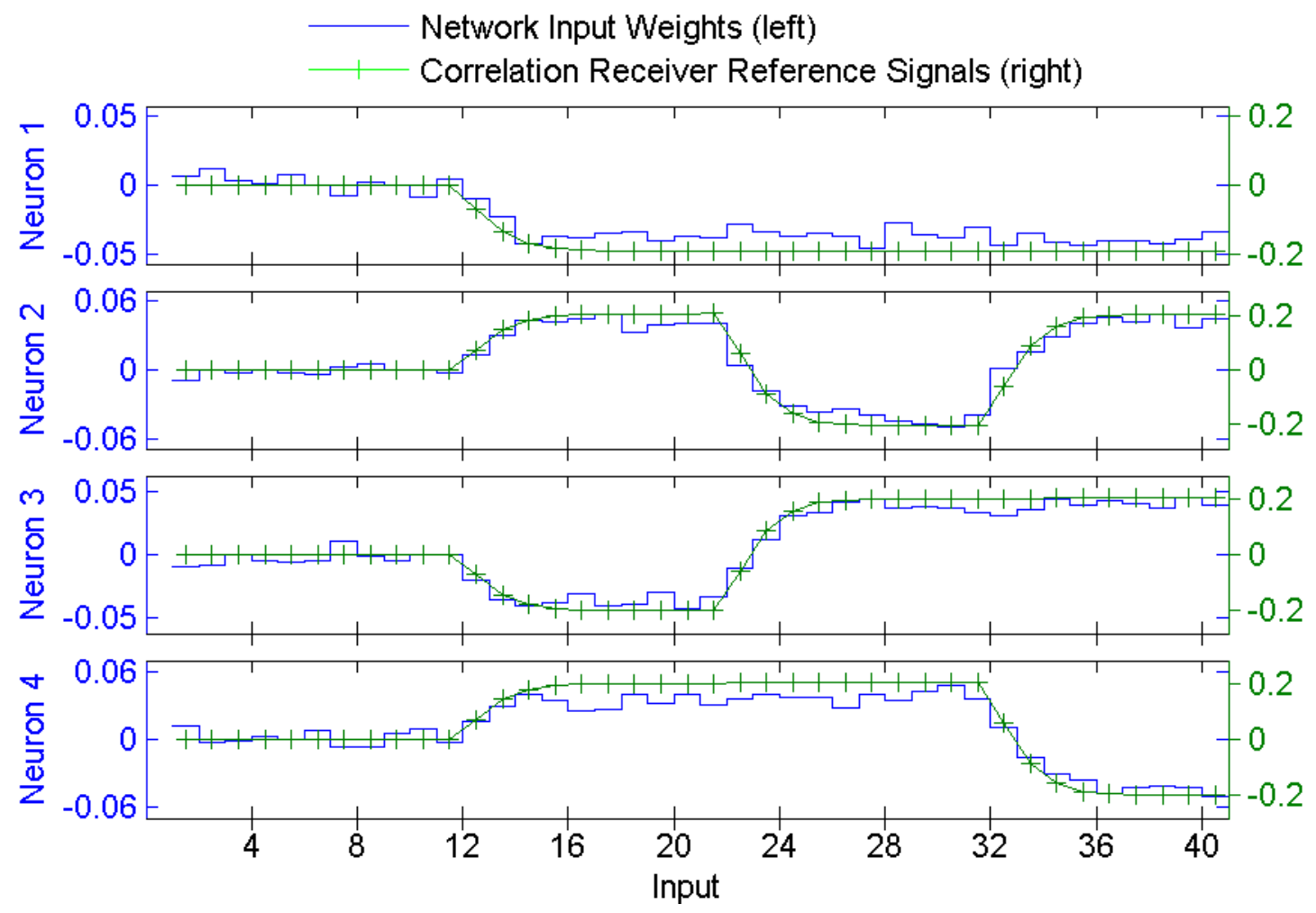

Figure 4-4 Input Weights Compared with Correlation Receiver Reference Signals.

The input weights are from the $1^{\text {st }}$ network of Figure 4-3 to each of the 4 neurons. 


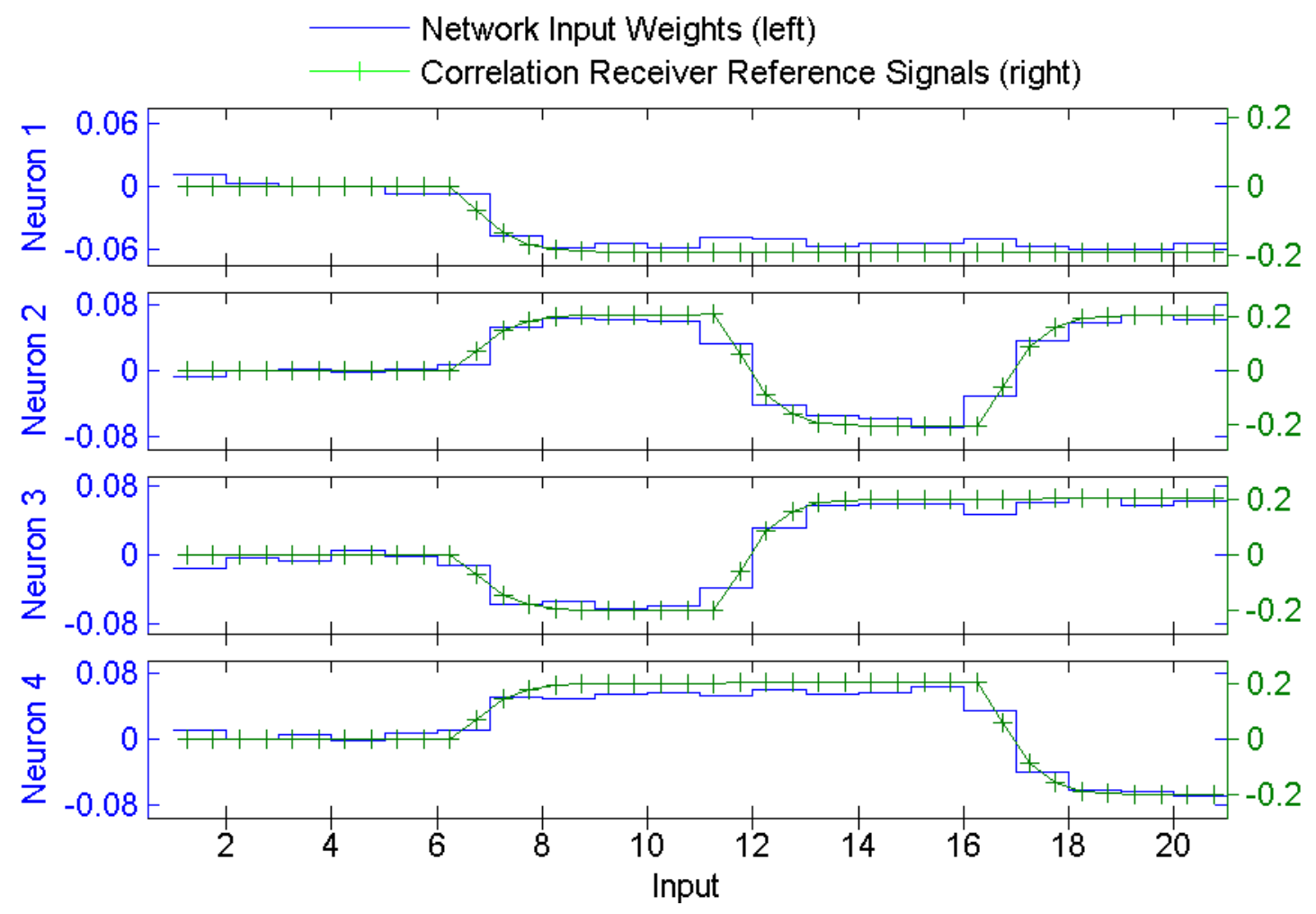

Figure 4-5 Input weights for the $4^{\text {th }}$ network of Figure 4-3 


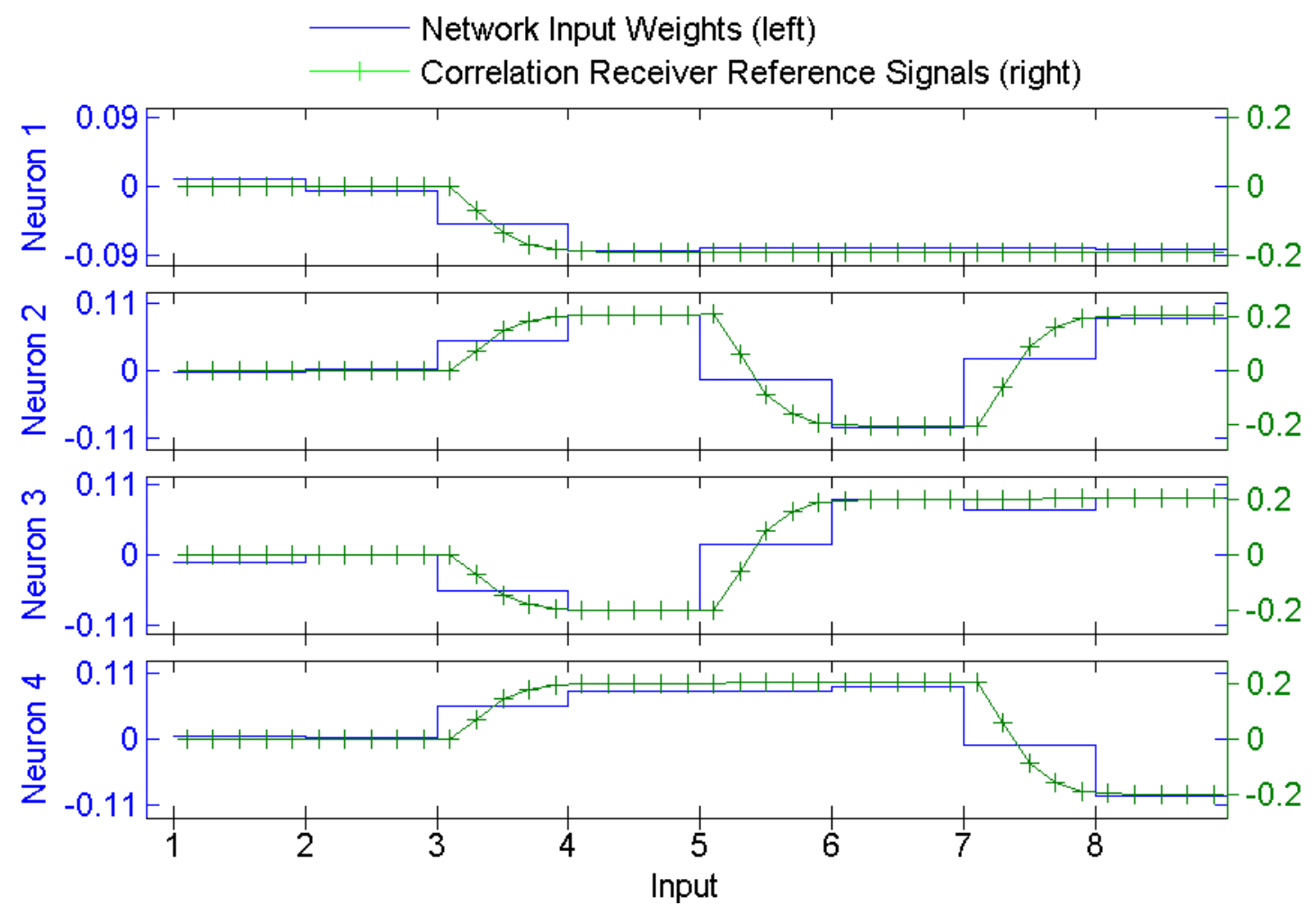

Figure 4-6 Input weights for the $7^{\text {th }}$ network of Figure 4-3 


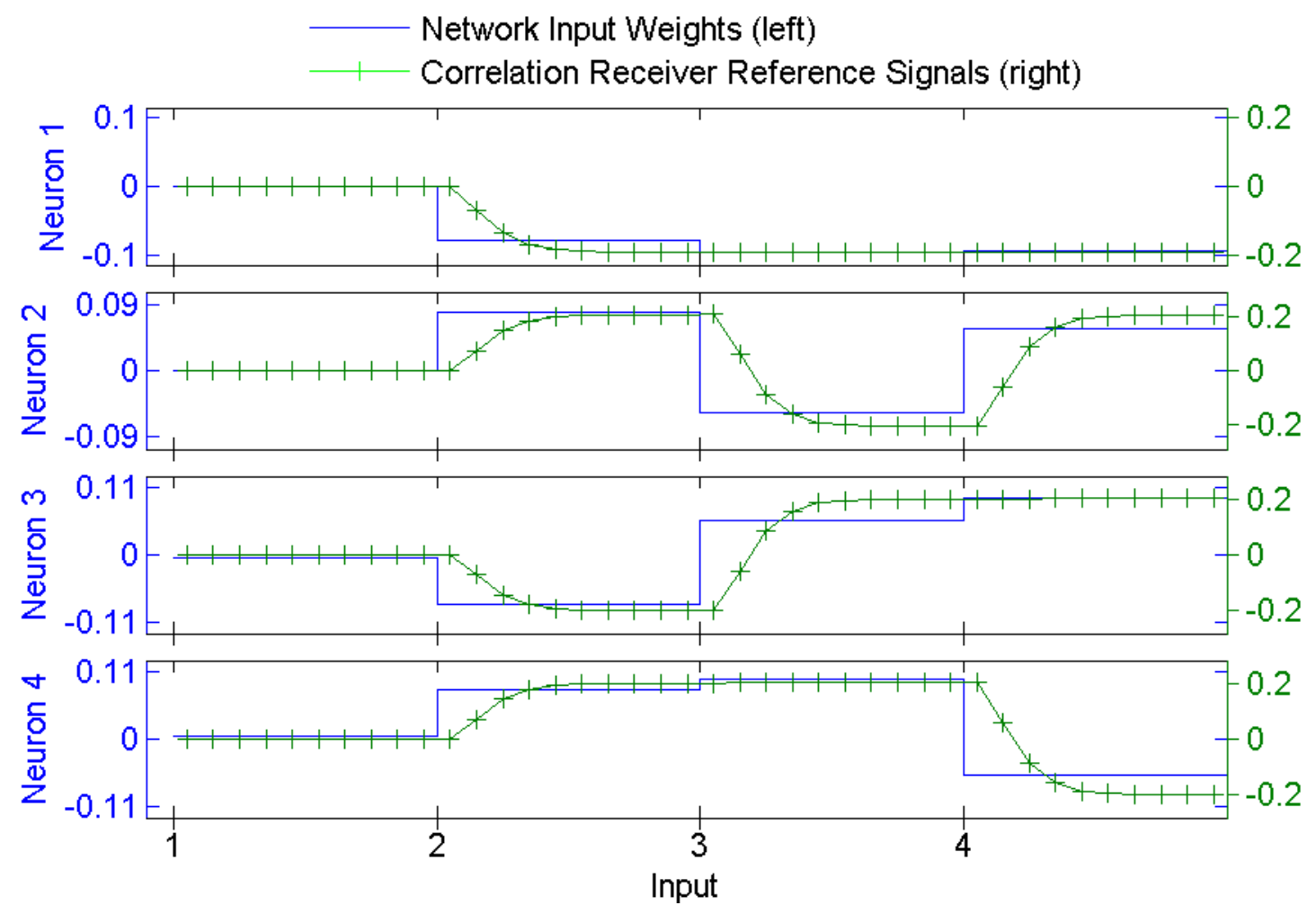

Figure 4-7 Input Weights for the $10^{\text {th }}$ network of Figure 4-3

The degraded performance of the single layer linear networks with smaller input sizes can be attributed to the reduction in information available to the network at each decision point. The network must use the same connection weight for all sampled data points that are fed through a particular input. The sampled points for a single transmitted symbol must be presented $N$ points at a time where $N$ is the number of inputs to the network. When the number of inputs is less than the total number of samples that make up a symbol, which is 40 in this case, then each input will receive multiple data points from a single sampled symbol.

For the case with an input size of four, each input will receive the sampled data points for a single bit of the sampled symbol. Each of these sampled data points are fed to the network one at a time, and the output of the network is averaged over the entire time it takes 
for a symbol to be presented to the network. Figure 4-4 above shows that the ideal weights are not uniform over an entire sampled bit from the code set. The architecture of the single layer linear networks used in this simulation prevents the networks from weighting a single input differently over time.

One way to address this is to change the post-processing method from a flat average to a weighted average. Performance would be expected to improve the greatest for networks that have the same number of inputs as bits contained in each symbol. The same trained networks simulated in Figure 4-3 were re-simulated with a weighted post-processing and the results are shown in Figure 4-8.

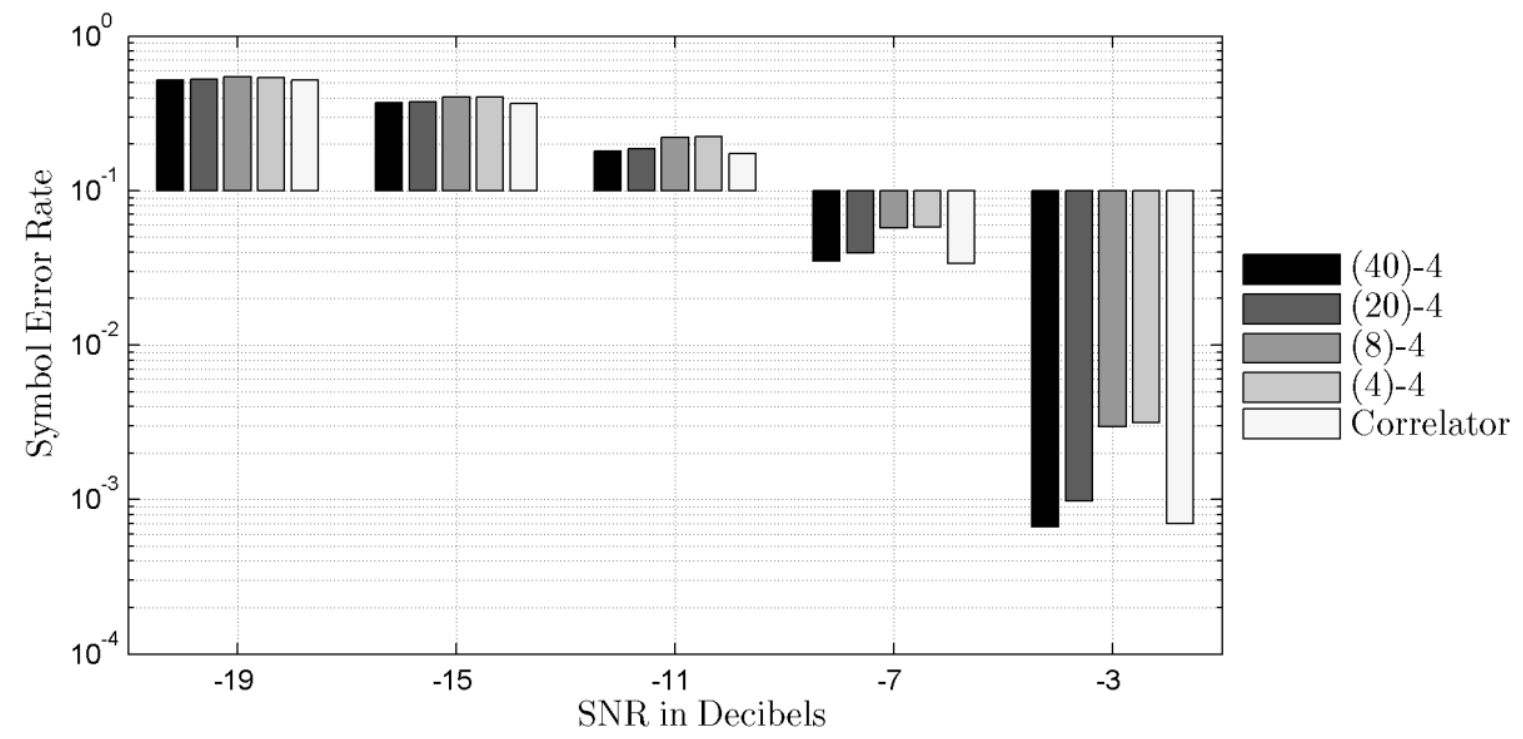

Figure 4-8 Single Layer Linear Network Simulation Results with Weighted Post Processing For AWGN with 4 Symbols. The exact same parameters were used as in Figure 4-3 


\begin{tabular}{|ccrrrr|}
\multicolumn{7}{c}{ SNR } \\
\hline Receiver & $\mathbf{- 1 9 d B}$ & $\mathbf{- 1 5 d B}$ & $\mathbf{- 1 1 d B}$ & $\mathbf{- 7 d B}$ & \multicolumn{1}{c|}{$\mathbf{d B}$} \\
\hline$(40)-4$ & 0.52174 & 0.37005 & 0.17997 & 0.03498 & 0.00067 \\
$(20)-4$ & 0.52244 & 0.37619 & 0.18741 & 0.03922 & 0.00098 \\
$(8)-4$ & 0.54756 & 0.40134 & 0.22097 & 0.05732 & 0.00295 \\
$(4)-4$ & 0.53750 & 0.40248 & 0.22352 & 0.05801 & 0.00317 \\
correlator & 0.51756 & 0.36649 & 0.17379 & 0.03379 & 0.00070 \\
\hline \# Symbols Tested & 17,200 & 20,200 & 29,000 & 42,200 & 60,000 \\
\hline
\end{tabular}

Table 4-6 SER Data for Figure 4-8

The network with four inputs saw a reduction in symbol error rate whereas the rest of the networks slightly increased. The ideal weighting of the outputs over time should be related to the channel characteristics that the transmission signal passes through on the way to the receiver. For practical applications, an estimation of the channel's transfer function may result in a good tradeoff between the performance of a correlation receiver, and the reduced computation afforded by a network with an equal number of inputs as bits in each symbol.

\subsubsection{Single Layer Linear Recurrent Network Simulations}

The results of adding a recurrent connection to the single layer linear network are shown in Figure 4-10. The gradient calculation for the simulations shown was not the true gradient, but rather an approximation. Simulations of recurrent networks in the Matlab environment took considerably more time than much larger non-recurrent networks. In simulations not included in this thesis, using the true gradient did not result in considerable performance benefits, but did require a much larger amount of computing time. Thus, an approximation to the true gradient, as described in section 2.3.8, is used in all recurrent simulations in this thesis. 


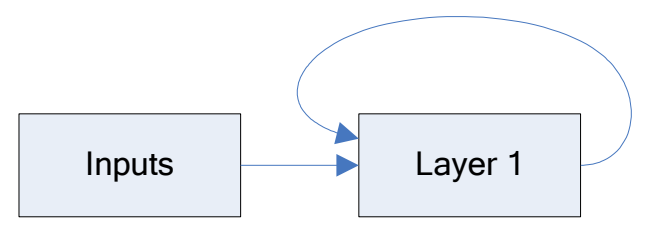

Figure 4-9 Layer Connections for Neural Networks of Figure 4-10

\begin{tabular}{|c|c|c|c|c|}
\hline Parameter & Value & Value & Value & Value \\
\hline Network Identifier & $(40)-4 \mathrm{R}$ & $(20)-4 \mathrm{R}$ & $(8)-4 \mathrm{R}$ & $(4)-4 \mathrm{R}$ \\
\hline Number if Inputs & 40 & 20 & 8 & 4 \\
\hline Layer 1 Neurons & 4 & 4 & 4 & 4 \\
\hline Layer 1 Transfer Function & SLINS & SLINS & SLINS & SLINS \\
\hline Training Function & $G D X$ & $G D X$ & GDX & GDX \\
\hline Initial Learning Rate & 0.05 & 0.05 & 0.05 & 3 \\
\hline Number of Networks & 3 & 3 & 3 & 1,200 Symbols \\
\hline Training Batch Size & 1,200 Symbols & 1,200 Symbols & 1,200 Symbols & $-11,-7,-3 \mathrm{~dB}$ \\
\hline Training SNR(s) & $-11,-7,-3 \mathrm{~dB}$ & $-11,-7,-3 \mathrm{~dB}$ & $-11,-7,-3 \mathrm{~dB}$ & 150 \\
\hline $\begin{array}{c}\text { Training Epochs per } \\
\text { Training Session }\end{array}$ & 150 & 150 & 150 & 2 \\
\hline \# of Training Sessions & 2 & 2 & 2 & \\
\hline
\end{tabular}

Table 4-7 Training and Test Parameters for Figure 4-10 


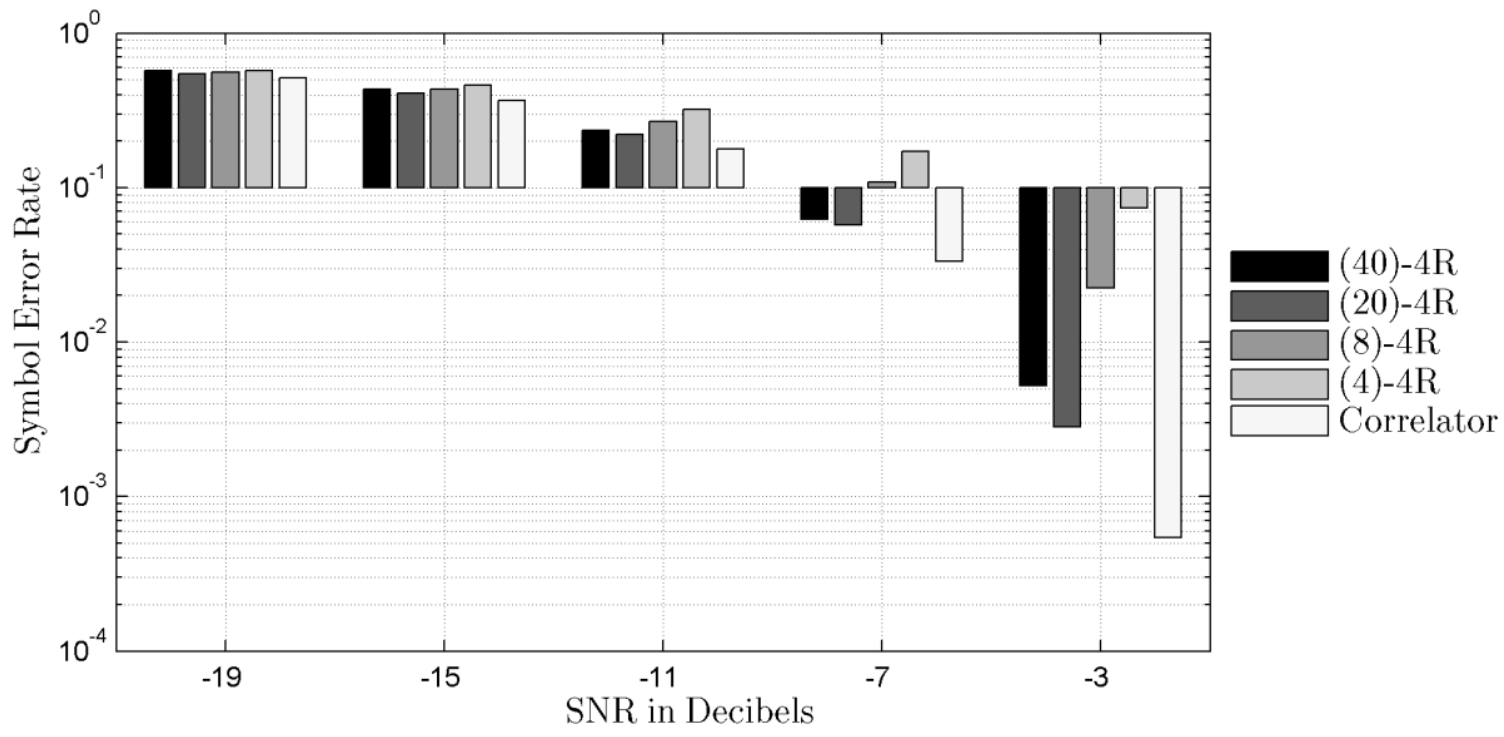

Figure 4-10 Single Layer Linear Recurrent Network Simulation Results for AWGN with 4 Symbols

\begin{tabular}{|cccccr|}
\hline \multicolumn{7}{c}{ SNR } \\
\hline Receiver & $\mathbf{- 1 9 d B}$ & $\mathbf{- 1 5 d B}$ & $\mathbf{- 1 1 d B}$ & $\mathbf{- 7 d B}$ & -3dB \\
\hline$(40)-4 R$ & 0.56841 & 0.43130 & 0.23423 & 0.06250 & 0.00520 \\
$(20)-4 R$ & 0.54749 & 0.40832 & 0.22091 & 0.05699 & 0.00282 \\
$(8)-4 R$ & 0.55703 & 0.43478 & 0.26909 & 0.10844 & 0.02245 \\
$(4)-4 R$ & 0.57170 & 0.46236 & 0.32227 & 0.17176 & 0.07346 \\
correlator & 0.51419 & 0.36672 & 0.17807 & 0.03338 & 0.00055 \\
\hline \# Symbols Tested & 102,600 & 102,600 & 102,600 & 102,600 & 102,600 \\
\hline
\end{tabular}

Table 4-8 SER Data For Figure 4-10

The recurrent networks' performance is worse than the results of the single layer linear networks. A modification to the post-processing of the recurrent network's outputs results in improved performance for the networks that are fed a single symbol portions at a time. The post-processing method used in Figure 4-10 was a simple flat average of the network's output for a single symbol. Note the reduced symbol error rate in Figure 4-11. The exact same trained networks of the previous figure were used, but the post-processing method more 
heavily weighted the output of the network as more inputs were presented for a specific symbol.

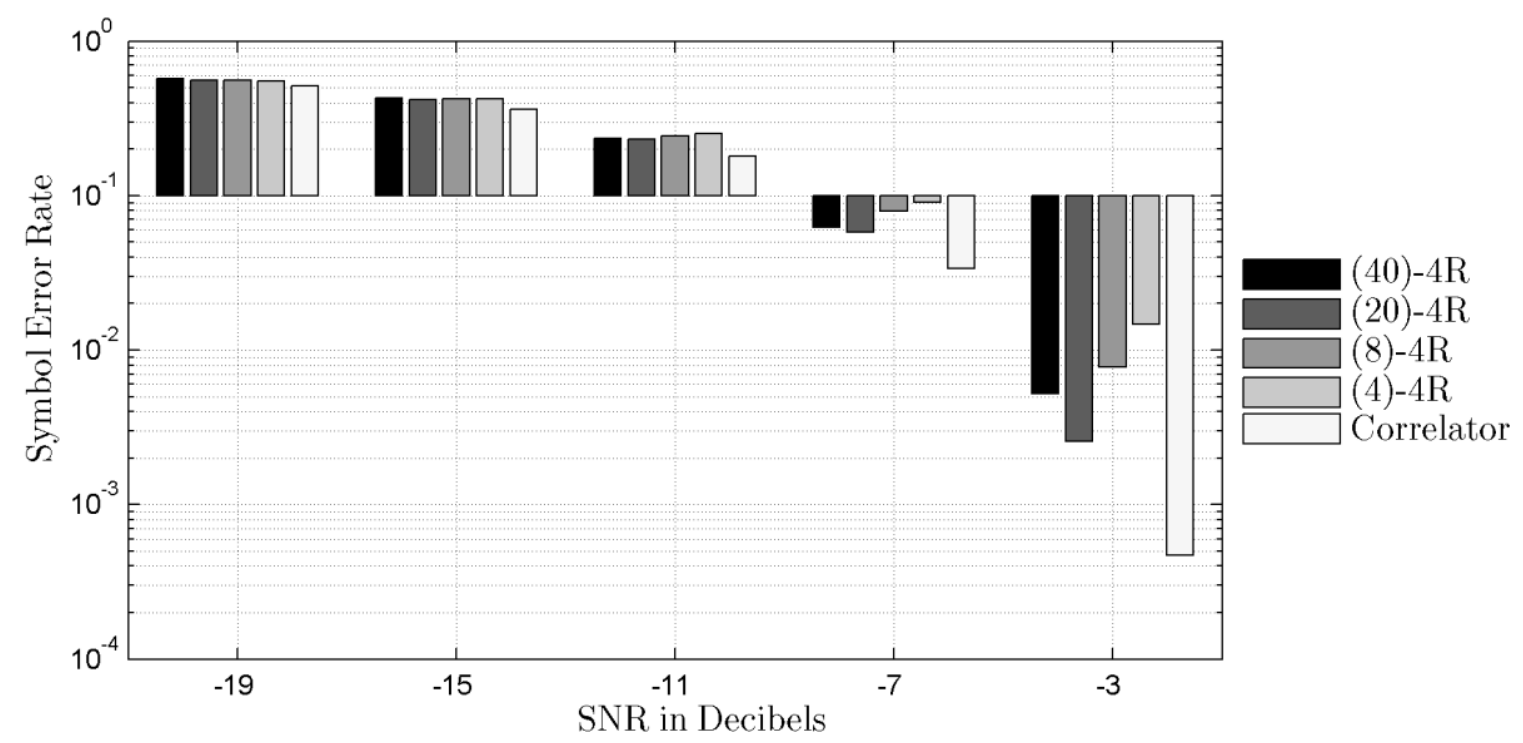

Figure 4-11 Single Layer Linear Recurrent Network Simulation Results with Weighted Post Processing For AWGN with 4 Symbols. The exact same parameters were used as in Figure 4-10.

\begin{tabular}{|cccrrr|}
\hline \multicolumn{7}{c}{ SNR } \\
\hline Receiver & $\mathbf{- 1 9 d B}$ & $\mathbf{- 1 5 d B}$ & $\mathbf{- 1 1 d B}$ & $\mathbf{- 7 d B}$ & -3dB \\
\hline$(40)-4 R$ & 0.57102 & 0.42613 & 0.23473 & 0.06258 & 0.00520 \\
$(20)-4 \mathrm{R}$ & 0.55754 & 0.41592 & 0.23025 & 0.05787 & 0.00256 \\
$(8)-4 \mathrm{R}$ & 0.56102 & 0.42148 & 0.24376 & 0.07897 & 0.00778 \\
(4)-4R & 0.55146 & 0.42070 & 0.25162 & 0.09054 & 0.01467 \\
correlator & 0.51314 & 0.36090 & 0.17938 & 0.03388 & 0.00047 \\
\hline \# Symbols Tested & 21,000 & 21,000 & 21,000 & 26,000 & 36,000 \\
\hline
\end{tabular}

Table 4-9 SER Data for Figure 4-11

The performance of the networks with input sizes of 4 and 8 improved, whereas the networks with input sizes of 20 slightly degraded. Further refinement of the post-processing method would most likely improve performance even more, although performance is expected to only approach that of the correlation receiver. No other recurrent network 
simulations were tested with AWGN noise due to the relatively poor performance and increased computation penalty.

\subsubsection{Single Layer Non-Linear Network Simulations}

Figure 4-13 and Figure 4-15 show the results of using a single nonlinear layer with AWGN corrupting noise for different input sizes.

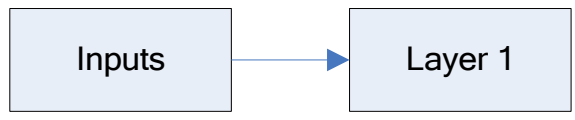

Figure 4-12 Layer Connections for Neural Networks of Figure 4-15

\begin{tabular}{|c|c|c|c|c|}
\hline Parameter & Value & Value & Value & Value \\
\hline Network Identifier & $(40)-4$ & $(20)-4$ & $(8)-4$ & $(4)-4$ \\
\hline Number if Inputs & 40 & 20 & 8 & 4 \\
\hline Layer 1 Neurons & 4 & 4 & 4 & $S I G$ \\
\hline Layer 1 Transfer Function & $S I G$ & $S I G$ & $G D X$ & $G D X$ \\
\hline Training Function & $G D X$ & $G D X$ & 0.05 & 5 \\
\hline Initial Learning Rate & 0.05 & 0.05 & 5 & 5 \\
\hline Number of Networks & 5 & 3,600 Symbols & 3,600 Symbols & 3,600 Symbols \\
\hline Training Batch Size & 3,600 Symbols & $-11,-7,-3 \mathrm{~dB}$ & $-11,-7,-3 \mathrm{~dB}$ & $-11,-7,-3 \mathrm{~dB}$ \\
\hline Training SNR(s) & $-11,-7,-3 \mathrm{~dB}$ & 200 & 200 & 200 \\
\hline $\begin{array}{c}\text { Training Epochs per } \\
\text { Training Session }\end{array}$ & 200 & 2 & 2 & 2 \\
\hline \# of Training Sessions & 2 & &
\end{tabular}

Table 4-10 Training and Test Parameters for Figure 4-15 


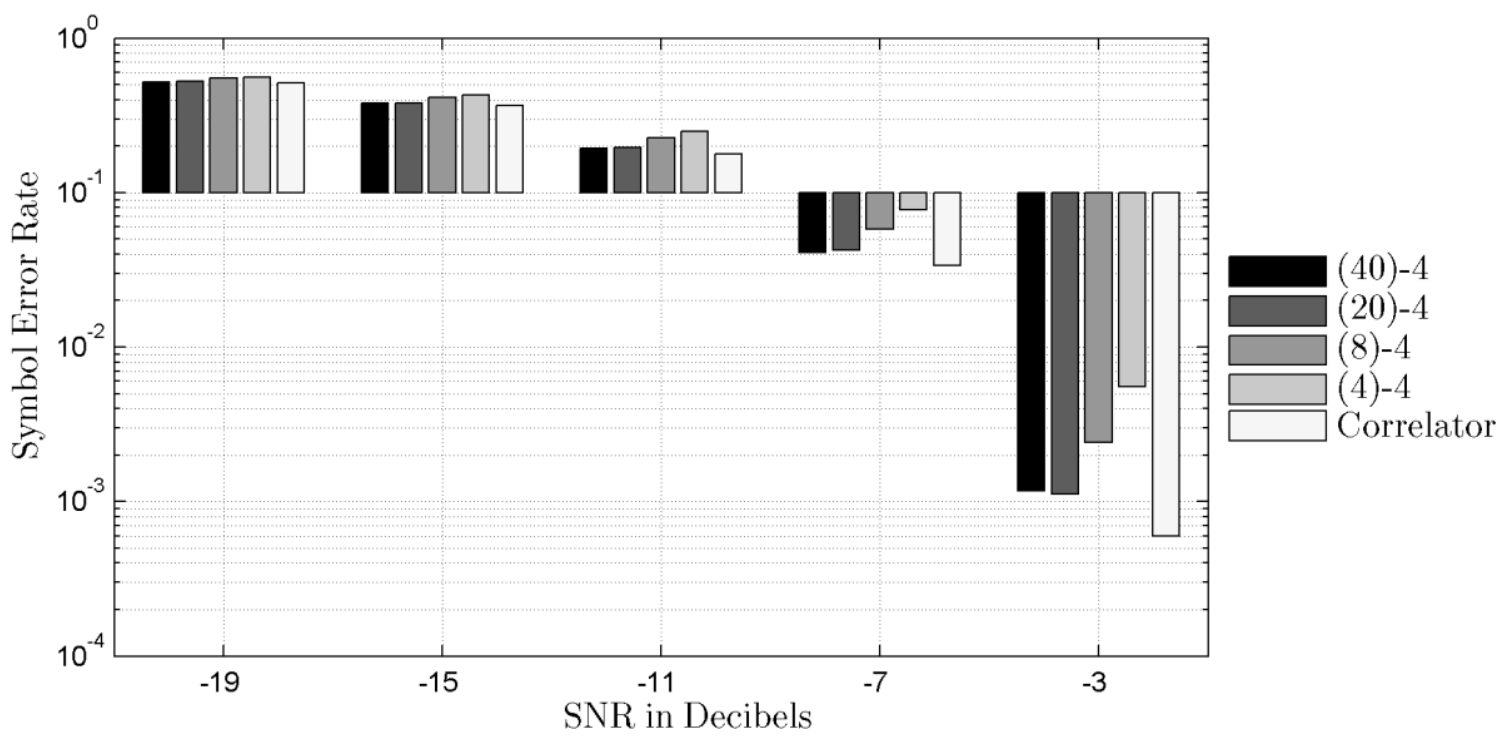

Figure 4-13 Single Layer SIG Network Simulation Results for AWGN with 4 Symbols

\begin{tabular}{|cccccr|}
\hline \multicolumn{7}{c}{ SNR } \\
\hline Receiver & $\mathbf{- 1 9 d B}$ & $\mathbf{- 1 5 d B}$ & $\mathbf{- 1 1 d B}$ & $\mathbf{- 7 d B}$ & $\mathbf{- 3 d B}$ \\
\hline$(40)-4$ & 0.52118 & 0.37936 & 0.19271 & 0.04094 & 0.00117 \\
$(20)-4$ & 0.52640 & 0.38099 & 0.19483 & 0.04264 & 0.00112 \\
$(8)-4$ & 0.55008 & 0.41249 & 0.22642 & 0.05794 & 0.00242 \\
$(4)-4$ & 0.55622 & 0.42675 & 0.24928 & 0.07721 & 0.00553 \\
correlator & 0.51341 & 0.36486 & 0.17784 & 0.03355 & 0.00060 \\
\hline \# Symbols Tested & 182,600 & 182,600 & 182,600 & 182,600 & 182,600 \\
\hline
\end{tabular}

Table 4-11 SER Data for Figure 4-13

Performance for the SIG networks with input sizes of 40 and 20 were worse than with the LIN networks of Figure 4-3. The performance of the networks with input sizes of 8 and 4 were slightly better.

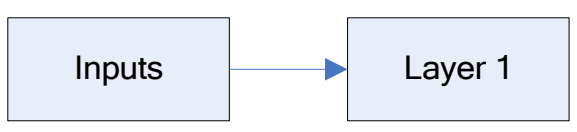

Figure 4-14 Layer Connections for Neural Networks of Figure 4-15 


\begin{tabular}{|c|c|c|c|c|}
\hline Parameter & Value & Value & Value & Value \\
\hline Network Identifier & $(40)-4$ & $(20)-4$ & $(8)-4$ & $(4)-4$ \\
\hline Number if Inputs & 40 & 20 & 8 & 4 \\
\hline Layer 1 Neurons & 4 & 4 & 4 & $R B$ \\
\hline Layer 1 Transfer Function & $R B$ & $R B$ & $R B$ & $G D X$ \\
\hline Training Function & $G D X$ & $G D X$ & 0.05 & 3 \\
\hline Initial Learning Rate & 0.05 & 0.05 & 3 & 3,600 Symbols \\
\hline Number of Networks & 3 & 3 & 3,600 Symbols & $-11,-7,-3 \mathrm{~dB}$ \\
\hline Training Batch Size & 3,600 Symbols & 3,600 Symbols & $-11,-7,-3 \mathrm{~dB}$ & 200 \\
\hline Training SNR(s) & $-11,-7,-3 \mathrm{~dB}$ & $-11,-7,-3 \mathrm{~dB}$ & 200 & 2 \\
\hline $\begin{array}{c}\text { Training Epochs per } \\
\text { Training Session }\end{array}$ & 200 & 200 & 2 & \\
\hline \# of Training Sessions & 2 & 2 & & 2 \\
\hline
\end{tabular}

Table 4-12 Training and Test Parameters for Figure 4-15

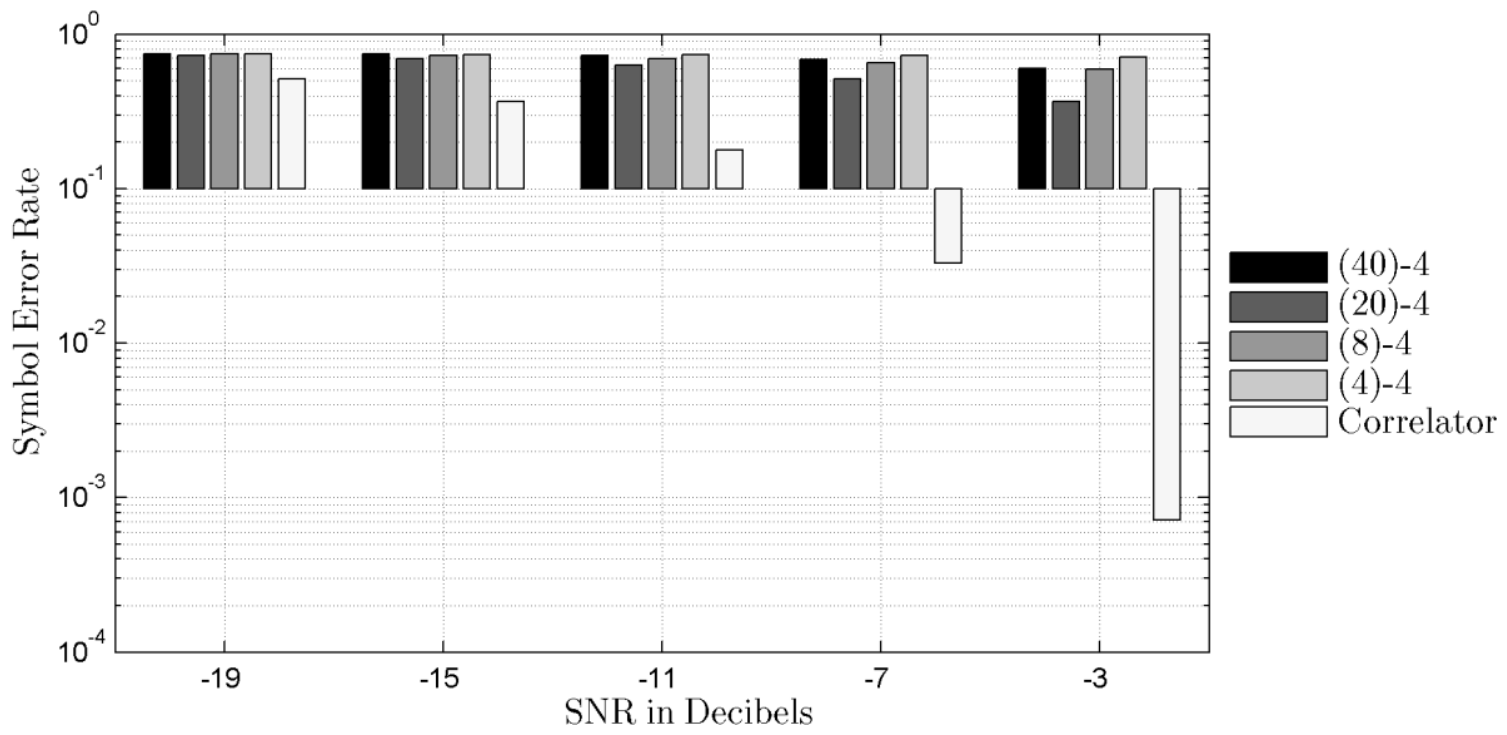

Figure 4-15 Single Layer RB Network Simulation Results for AWGN with 4 Symbols 
SNR

\begin{tabular}{|ccrrrr|}
\hline Receiver & $\mathbf{- 1 9 d B}$ & $\mathbf{- 1 5 d B}$ & \multicolumn{1}{c|}{$\mathbf{1 1 d B}$} & $\mathbf{- 7 d B}$ & -3dB \\
\hline$(40)-4$ & 0.74768 & 0.74214 & 0.72460 & 0.68479 & 0.59890 \\
$(20)-4$ & 0.72887 & 0.69637 & 0.63029 & 0.51544 & 0.36735 \\
$(8)-4$ & 0.74431 & 0.72831 & 0.69429 & 0.65268 & 0.59487 \\
$(4)-4$ & 0.74253 & 0.73427 & 0.73211 & 0.72598 & 0.70674 \\
correlator & 0.51541 & 0.36408 & 0.17826 & 0.03275 & 0.00071 \\
\hline \# Symbols Tested & 82,600 & 82,600 & 82,600 & 82,600 & 82,600 \\
\hline
\end{tabular}

Table 4-13 SER Data For Figure 4-15

The single layer nonlinear networks using a radial basis transfer function did not perform well.

\subsubsection{Multi-Layer Non-Linear Network Simulations}

\section{Varying Input Size}

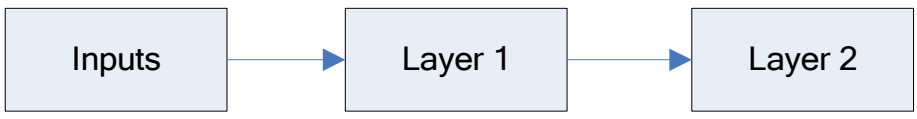

Figure 4-16 Layer Connections for Neural Networks of Figure 4-17

\begin{tabular}{|c|c|c|c|c|}
\hline Parameter & Value & Value & Value & Value \\
\hline Network Identifier & $(40)-16-4$ & $(20)-16-4$ & $(8)-16-4$ & $(4)-16-4$ \\
\hline Number if Inputs & 40 & 20 & 8 & 4 \\
\hline Layer 1 Neurons & 16 & 16 & 16 & 16 \\
\hline Layer 1 Transfer Function & $S I G$ & $S I G$ & $S I G$ & 4 \\
\hline Layer 2 Neurons & 4 & 4 & 4 & $S I G$ \\
\hline Layer 2 Transfer Function & $S I G$ & $S I G$ & $G D X$ & $G D X$ \\
\hline Training Function & $G D X$ & $G D X$ & 0.05 & 0.05 \\
\hline Initial Learning Rate & 0.05 & 0.05 & 5 & 5 \\
\hline Number of Networks & 5 & 5 & $2,400 \mathrm{Symbols}$ & 2,400 Symbols \\
\hline Training Batch Size & $2,400 \mathrm{Symbols}$ & 2,400 Symbols & $-11,-7,-3 \mathrm{~dB}$ & $-11,-7,-3 \mathrm{~dB}$ \\
\hline Training SNR(s) & $-11,-7,-3 \mathrm{~dB}$ & $-11,-7,-3 \mathrm{~dB}$ & 150 & 150 \\
\hline Training Epochs per & 150 & 150 & 3 & 3 \\
\hline Training Session & 3 & 3 & & \\
\hline \# of Training Sessions & & &
\end{tabular}

Table 4-14 Training and Test Parameters for Figure 4-17 


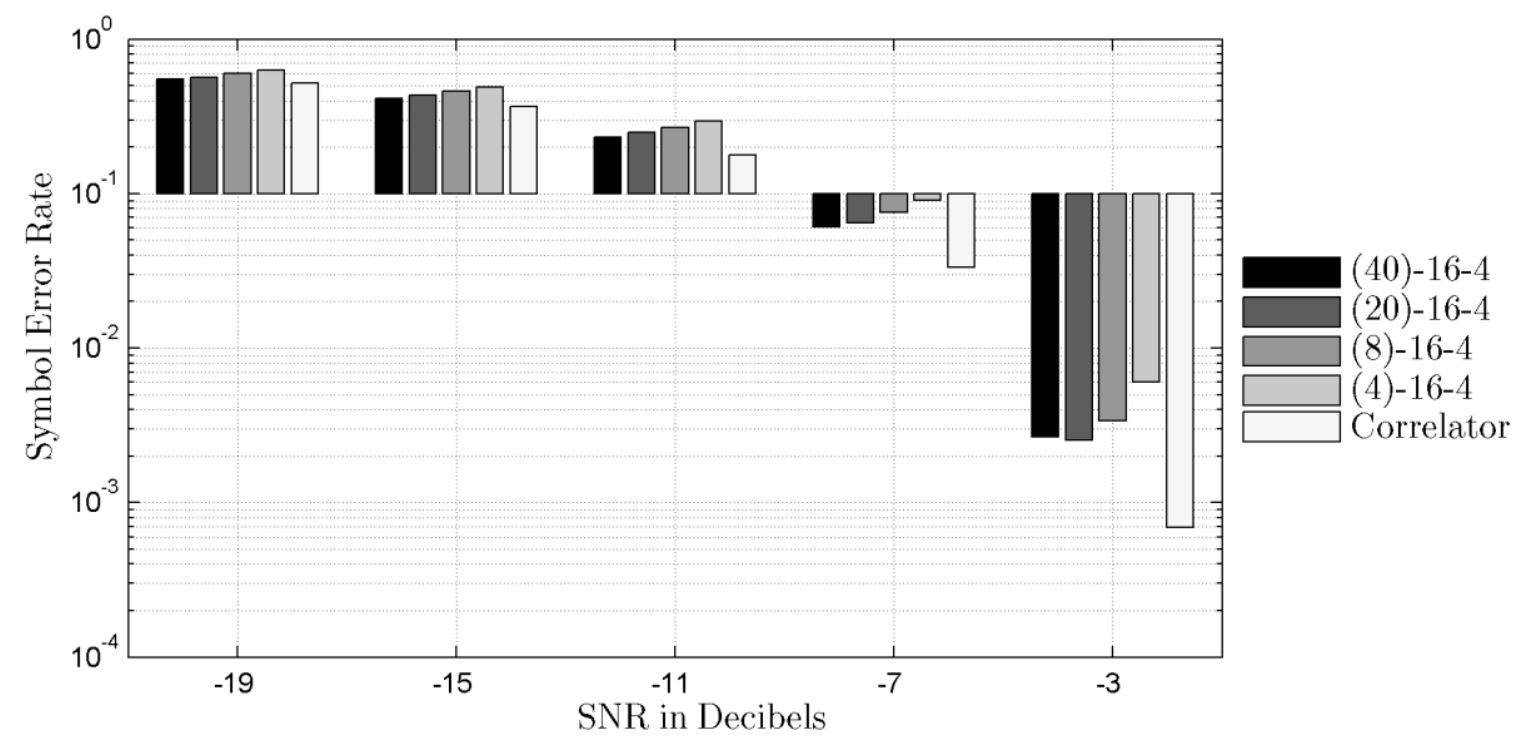

Figure 4-17 Varying Input Size of 2 layer SIG Networks for AWGN with 4 Symbols

\begin{tabular}{|cccccr|}
\hline \multicolumn{7}{c}{ SNR } \\
\hline Receiver & $\mathbf{- 1 9 d B}$ & $\mathbf{- 1 5 d B}$ & $\mathbf{- 1 1 d B}$ & $\mathbf{- 7 d B}$ & -3dB \\
\hline$(40)-16-4$ & 0.54953 & 0.41457 & 0.23224 & 0.06065 & 0.00267 \\
$(20)-16-4$ & 0.56263 & 0.43207 & 0.25025 & 0.06426 & 0.00254 \\
$(8)-16-4$ & 0.59693 & 0.45825 & 0.26896 & 0.07544 & 0.00339 \\
$(4)-16-4$ & 0.63130 & 0.48583 & 0.29430 & 0.09080 & 0.00607 \\
correlator & 0.51944 & 0.36428 & 0.17766 & 0.03342 & 0.00069 \\
\hline \# Symbols Tested & 36,000 & 43,200 & 67,200 & 100,000 & 100,000 \\
\hline
\end{tabular}

Table 4-15 SER Data for Figure 4-17

Performance of the 2 layer SIG networks degraded somewhat from the single layer SIG networks. 


\section{Varying Input Layer Size}

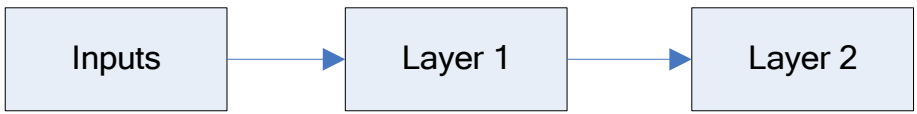

Figure 4-18 Layer Connections for Neural Networks of Figure 4-19

\begin{tabular}{|c|c|c|c|}
\hline Parameter & Value & Value & Value \\
\hline Network Identifier & $(40)-12-4$ & $(40)-8-4$ & $(40)-4-4$ \\
\hline Number if Inputs & 40 & 40 & 40 \\
\hline Layer 1 Neurons & 12 & 8 & 4 \\
\hline Layer 1 Transfer Function & $S I G$ & $S I G$ & $S I G$ \\
\hline Layer 2 Neurons & 4 & 4 & 4 \\
\hline Layer 2 Transfer Function & $S I G$ & $S I G$ & SIG \\
\hline Training Function & $G D X$ & $G D X$ & 0.05 \\
\hline Initial Learning Rate & 0.05 & 0.05 & 5 \\
\hline Number of Networks & 5 & 5 & 1,600 Symbols \\
\hline Training Batch Size & 1,600 Symbols & 1,600 Symbols & $-11,-7,-3,1 \mathrm{~dB}$ \\
\hline Training SNR(s) & $-11,-7,-3,1 \mathrm{~dB}$ & $-11,-7,-3,1 \mathrm{~dB}$ & 200 \\
\hline Training Epochs per & 200 & 200 & 2 \\
\hline Training Session & 2 & 2 & \\
\hline \# of Training Sessions & 200 &
\end{tabular}

Table 4-16 Training and Test Parameters for Figure 4-19

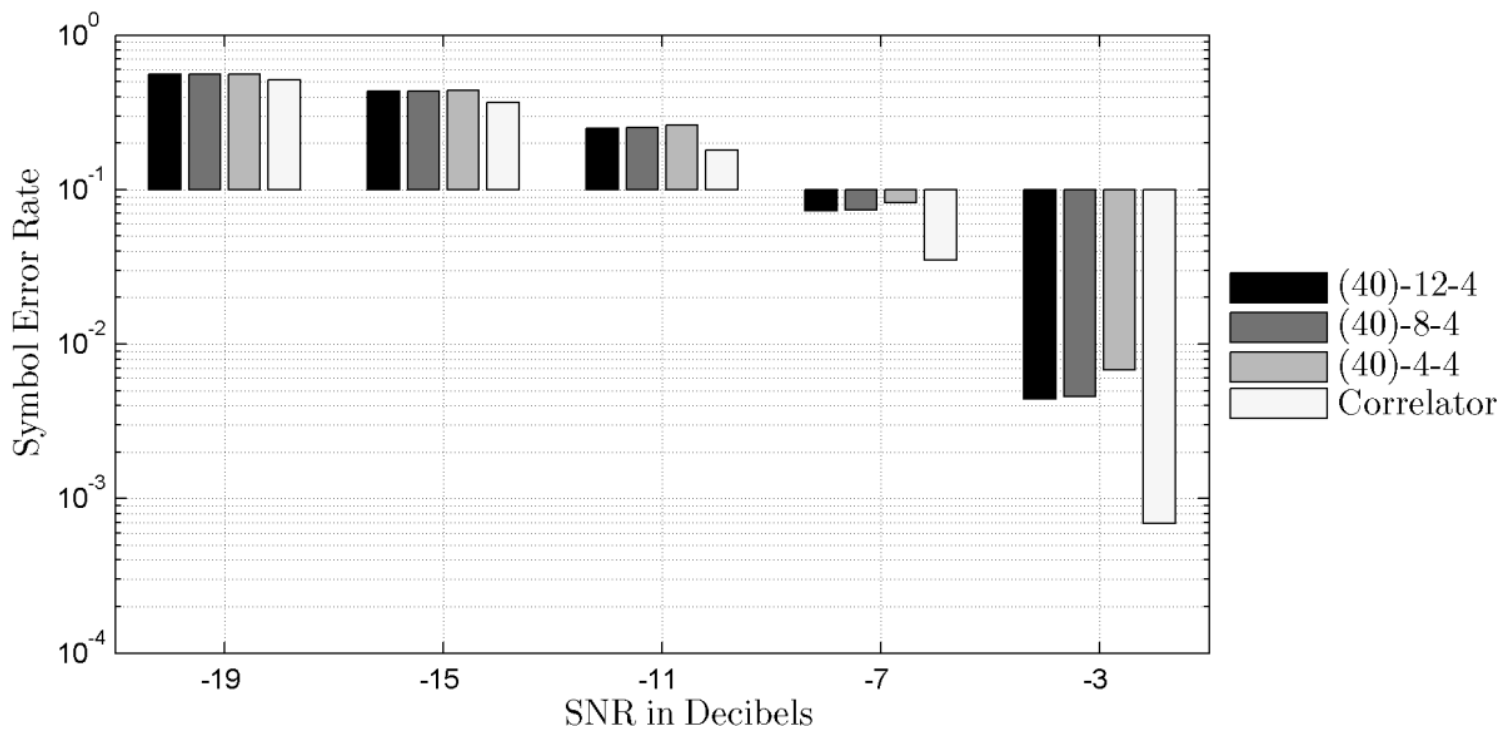

Figure 4-19 Varying Input Layer Size of 2 Layer SIG Networks for AWGN with 4 Symbols 


\section{SNR}

\begin{tabular}{|crrrrr|}
\hline Receiver & -19dB & \multicolumn{1}{c}{-15dB } & \multicolumn{1}{c|}{-11dB } & -7dB & -3dB \\
\hline$(40)-12-4$ & 0.55525 & 0.43183 & 0.25042 & 0.07315 & 0.00442 \\
$(40)-8-4$ & 0.55437 & 0.43100 & 0.25051 & 0.07404 & 0.00459 \\
$(40)-4-4$ & 0.56103 & 0.43883 & 0.26098 & 0.08246 & 0.00683 \\
correlator & 0.51143 & 0.36605 & 0.18018 & 0.03512 & 0.00069 \\
\hline \# Symbols Tested & 82,600 & 82,600 & 82,600 & 82,600 & 82,600 \\
\hline
\end{tabular}

Table 4-17 SER Data for Figure 4-19

The networks with an input layer size of 4 performed slightly worse than those with 8 and 12 neurons in the input layer. Performance of the networks with 8 and 12 input layer neurons performed equally well. 


\section{Cascade Networks}

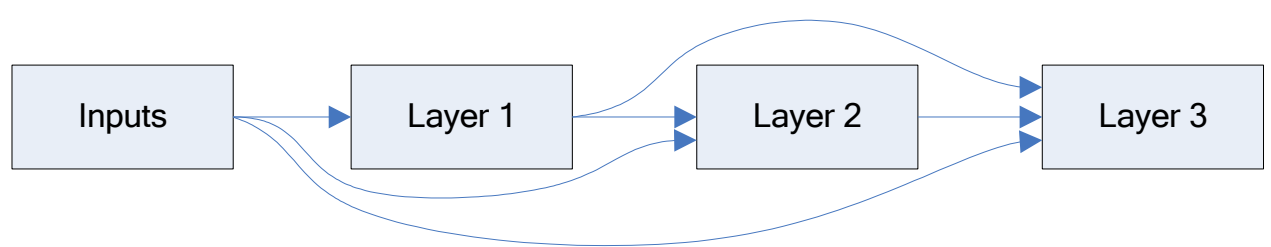

Figure 4-20 Layer Connections for Neural Networks of Figure 4-21

\begin{tabular}{|c|c|c|c|}
\hline Parameter & Value & Value & Value \\
\hline Network Identifier & $(40)-8-8-4$ casc & $(40)-4-4-4$ casc & $(40)-0-0-4$ casc \\
\hline Number if Inputs & 40 & 40 & 40 \\
\hline Layer 1 Neurons & 8 & 4 & 0 \\
\hline Layer 1 Transfer Function & $S I G$ & $S I G$ & SIG \\
\hline Layer 2 Neurons & 8 & 4 & SIG \\
\hline Layer 2 Transfer Function & $S I G$ & $S I G$ & 4 \\
\hline Layer 3 Neurons & 4 & 4 & LIN \\
\hline Layer 3 Transfer Function & LIN & LIN & 0.05 \\
\hline Training Function & $G D X$ & $G D X$ & 5 \\
\hline Initial Learning Rate & 0.05 & 0.05 & 1,200 Symbols \\
\hline Number of Networks & 5 & 5 & $-11,-7,-3 \mathrm{~dB}$ \\
\hline Training Batch Size & $1,200 \mathrm{Symbols}$ & $1,200 \mathrm{Symbols}$ & 100 \\
\hline Training SNR(s) & $-11,-7,-3 \mathrm{~dB}$ & $-11,-7,-3 \mathrm{~dB}$ & 2 \\
\hline Training Epochs per & 100 & 100 & 2 \\
\hline Training Session & 2 & & 2100 \\
\hline \# of Training Sessions & &
\end{tabular}

Table 4-18 Training and Test Parameters for Figure 4-21 


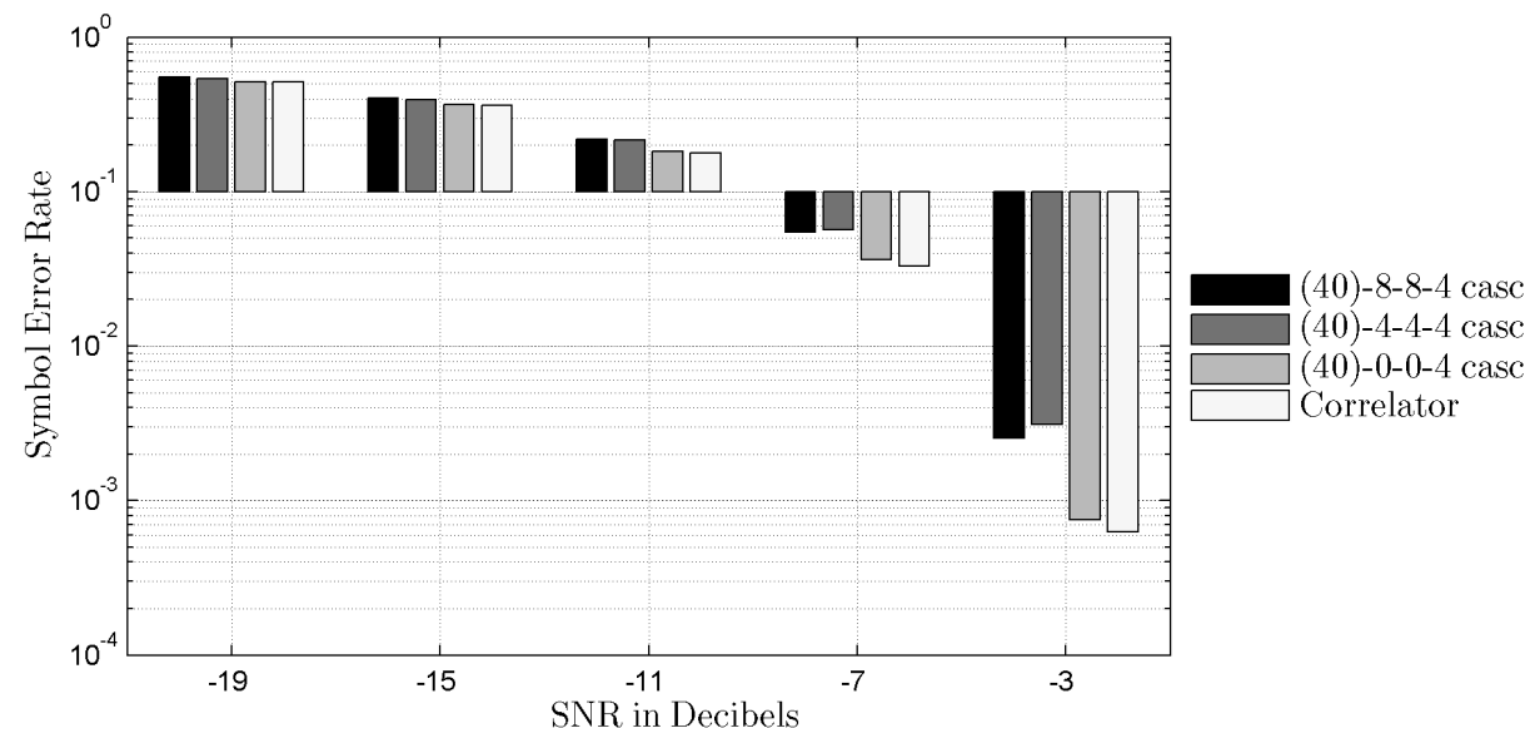

Figure 4-21 Varying Size of Cascaded Nonlinear Networks with Linear Output Layer for AWGN with 4 Symbols.

\begin{tabular}{|crrrrr|}
\multicolumn{7}{c}{ SNR } \\
\hline Receiver & $\mathbf{- 1 9 d B}$ & $\mathbf{- 1 5 d B}$ & $\mathbf{- 1 1 d B}$ & $\mathbf{- 7 d B}$ & -3dB \\
\hline (40)-8-8-4 casc & 0.55155 & 0.40344 & 0.21690 & 0.05476 & 0.00254 \\
$(40)-4-4-4$ casc & 0.53972 & 0.39533 & 0.21449 & 0.05638 & 0.00312 \\
(40)-0-0-4 casc & 0.51533 & 0.36780 & 0.18311 & 0.03614 & 0.00075 \\
correlator & 0.51018 & 0.36292 & 0.17731 & 0.03281 & 0.00063 \\
\hline \# Symbols Tested & 82,600 & 82,600 & 82,600 & 82,600 & 82,600 \\
\hline
\end{tabular}

Table 4-19 SER Data for Figure 4-21

The networks simulated in Figure 4-21 have the inputs connected to each layer. The first 2 non-linear layers are also connected to the linear output layer. SIG neurons were used for the first 2 layers. It was expected that the performance of the cascaded networks should approach that of the single layer linear networks because they each contain a single layer linear network. The reasoning would be that during training the connection weights to the non-linear layers would diminish while the input connections to the output linear layer would closely match the weights of the single layer linear networks. Figure 4-23 shows the results of 
implementing a pseudo cascade correlation algorithm for training the networks. The initial network consists of a layer of linear neurons. The connection weights are trained and then frozen. Afterwards one or more non-linear layers are added and the network is trained again.

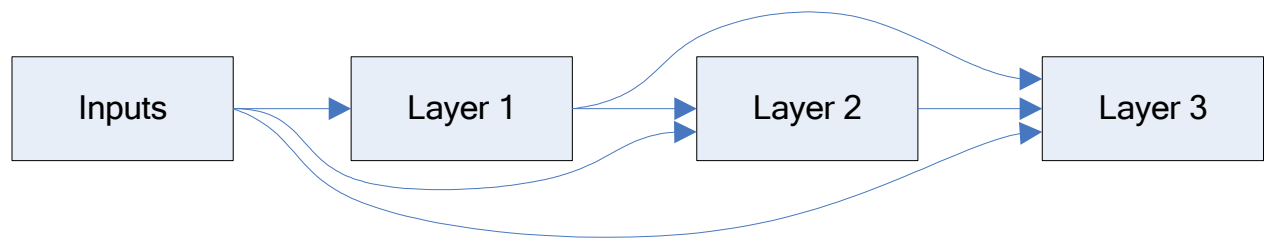

Figure 4-22 Layer Connections for Neural Networks of Figure 4-23

\begin{tabular}{|c|c|c|c|}
\hline Parameter & Value & Value & Value \\
\hline Network Identifier & $(40)-0-0-4$ casc & $(40)-0-4-4$ casc & $(40)-4-4-4$ casc \\
\hline Number if Inputs & 40 & 40 & 40 \\
\hline Layer 1 Neurons & 0 & 0 & 4 \\
\hline Layer 1 Transfer Function & $S I G$ & $S I G$ & $S I G$ \\
\hline Layer 2 Neurons & 0 & 4 & 4 \\
\hline Layer 2 Transfer Function & $S I G$ & $S I G$ & $4 G$ \\
\hline Layer 3 Neurons & 4 & 4 & $L I N$ \\
\hline Layer 3 Transfer Function & LIN & $L I N$ & $G D X$ \\
\hline Training Function & $G D X$ & $G D X$ & 0.05 \\
\hline Initial Learning Rate & 0.05 & 0.05 & 5 \\
\hline Number of Networks & 5 & 5 & 2,400 Symbols \\
\hline Training Batch Size & 2,400 Symbols & $2,400 \mathrm{Symbols}$ & $-11,-7,-3 \mathrm{~dB}$ \\
\hline Training SNR(s) & $-11,-7,-3 \mathrm{~dB}$ & $-11,-7,-3 \mathrm{~dB}$ & 100 \\
\hline $\begin{array}{c}\text { Training Epochs per } \\
\text { Training Session }\end{array}$ & 100 & 100 & 2 \\
\hline \# of Training Sessions & 2 & 2 & 23 \\
\hline
\end{tabular}

Table 4-20 Training and Test Parameters for Figure 4-23 


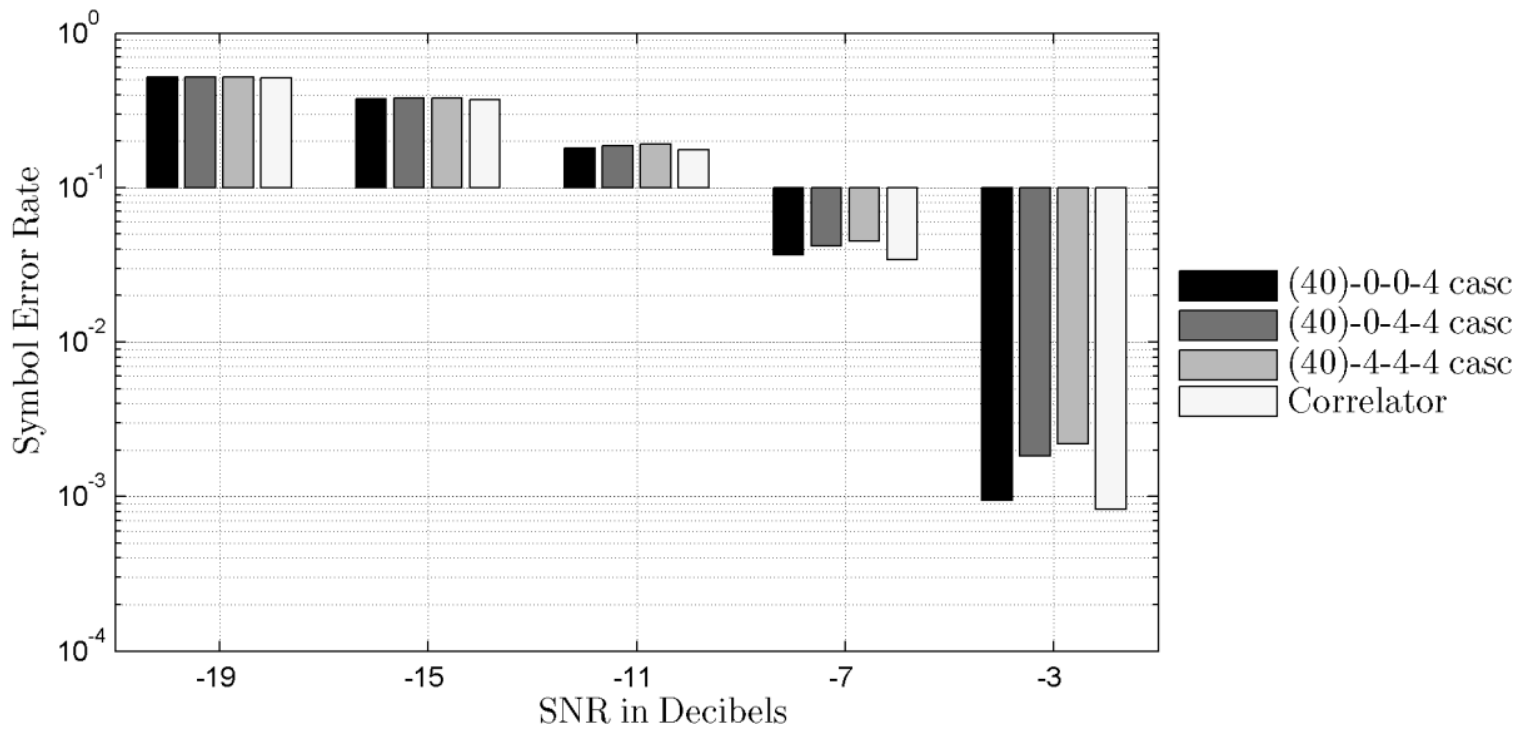

Figure 4-23 Pseudo Cascade Correlation Networks for AWGN with 4 Symbols

\begin{tabular}{|c|c|c|c|c|c|}
\hline & & & SNR & & \\
\hline Receiver & $-19 \mathrm{~dB}$ & $-15 \mathrm{~dB}$ & $-11 \mathrm{~dB}$ & $-7 \mathrm{~dB}$ & $-3 \mathrm{~dB}$ \\
\hline$(40)-0-0-4$ casc & 0.51808 & 0.37566 & 0.18040 & 0.03672 & 0.00095 \\
\hline (40)-0-4-4 casc & 0.51829 & 0.37816 & 0.18586 & 0.04189 & 0.00184 \\
\hline (40)-4-4-4 casc & 0.51816 & 0.37910 & 0.18996 & 0.04478 & 0.00219 \\
\hline correlator & 0.51202 & 0.37052 & 0.17594 & 0.03403 & 0.00083 \\
\hline \# Symbols Tested & 20,800 & 21,200 & 22,400 & 32,000 & 40,000 \\
\hline
\end{tabular}

Table 4-21 SER Data for Figure 4-23

The resulting cascaded networks perform better than when all connection weights were trained at once. 


\section{Varying Training Algorithm}

Figure 4-25 shows the results of training a single layer linear network with 3 different training algorithms. The first algorithm is a version of the conjugate gradient algorithm. The second is the Levenberg-Marquardt algorithm. The last algorithm is gradient descent with momentum and an adaptive learning rate.

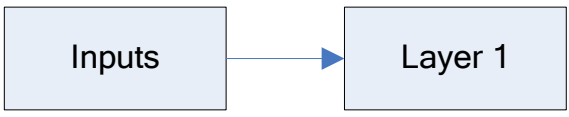

Figure 4-24 Layer Connections for Neural Networks of Figure 4-25 and Figure 4-26

\begin{tabular}{|c|c|c|c|}
\hline Parameter & Value & Value & Value \\
\hline Network Identifier & (40)-4 CGP & (40)-4 LM & (40)-4 GDX \\
\hline Number if Inputs & 40 & 40 & 40 \\
\hline Layer 1 Neurons & 4 & 4 & 4 \\
\hline Layer 1 Transfer Function & $S I G$ & $S I G$ & $S I G$ \\
\hline Training Function & $C G P$ & $L M$ & $G D X$ \\
\hline Initial Learning Rate & & & 0.05 \\
\hline Number of Networks & 5 & 5 & 5 \\
\hline Training Batch Size & 2,400 Symbols & 2,400 Symbols & 2,400 Symbols \\
\hline Training SNR(s) & $-11,-7,-3 \mathrm{~dB}$ & $-11,-7,-3 \mathrm{~dB}$ & $-11,-7,-3 \mathrm{~dB}$ \\
\hline $\begin{array}{l}\text { Training Epochs per } \\
\text { Training Session }\end{array}$ & 100 & 100 & 100 \\
\hline \# of Training Sessions & 2 & 2 & 2 \\
\hline
\end{tabular}

Table 4-22 Training and Test Parameters for Figure 4-25 


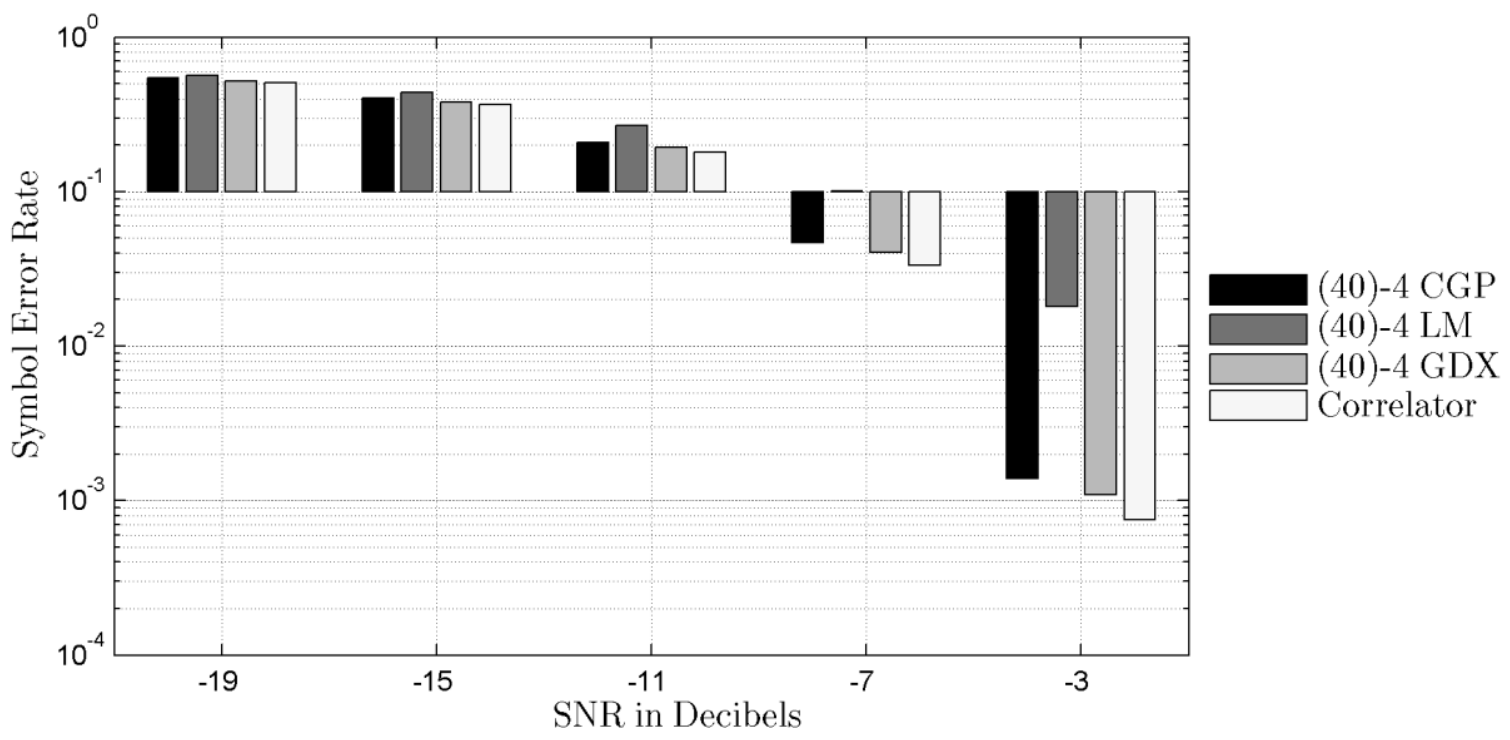

Figure 4-25 Varying the training algorithm of a single linear layer for AWGN with 4 symbols

\begin{tabular}{|cccccr|}
\multicolumn{7}{c}{ SNR } \\
\hline Receiver & $\mathbf{- 1 9 d B}$ & $\mathbf{- 1 5 d B}$ & $\mathbf{- 1 1 d B}$ & $\mathbf{- 7 d B}$ & -3dB \\
\hline (40)-4 CGP & 0.54508 & 0.40209 & 0.20856 & 0.04687 & 0.00138 \\
(40)-4 LM & 0.56711 & 0.44078 & 0.26598 & 0.10089 & 0.01812 \\
(40)-4 GDX & 0.51885 & 0.37816 & 0.19293 & 0.04054 & 0.00110 \\
Correlator & 0.50829 & 0.36398 & 0.17982 & 0.03332 & 0.00075 \\
\hline \# Symbols Tested & 32,600 & 42,200 & 65,200 & 100,000 & 100,000 \\
\hline
\end{tabular}

Table 4-23 SER Data for Figure 4-25

The training function GDX performed the best for this configuration. The poor performance of the Levenberg-Marquardt algorithm (LM) is most likely due to over fitting. Figure 4-26 shows the results of reducing the amount of training by setting the number of Training Epochs per Training Session to 20 instead of 100 for the LM training function. 


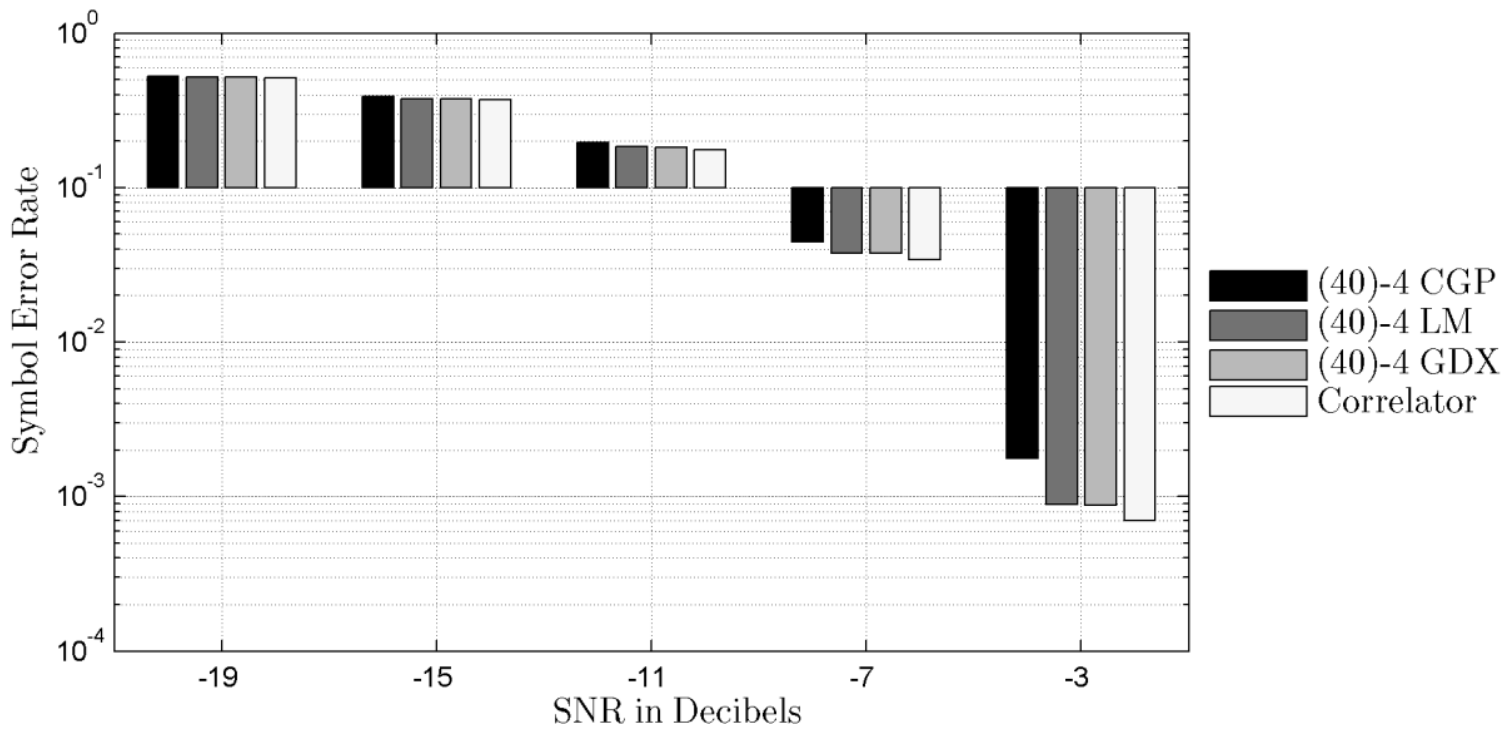

Figure 4-26 Same as Figure 4-25 with Reduced Training for the Levenberg-Marquardt Algorithm. The number of epochs per batch was reduced to 20 from 100.

\begin{tabular}{|cccccr|}
\multicolumn{7}{c}{ SNR } \\
\hline Receiver & $\mathbf{- 1 9 d B}$ & $\mathbf{- 1 5 d B}$ & $\mathbf{- 1 1 d B}$ & $\mathbf{- 7 d B}$ & -3dB \\
\hline (40)-4 CGP & 0.52690 & 0.38888 & 0.19568 & 0.04474 & 0.00177 \\
(40)-4 LM & 0.51789 & 0.37613 & 0.18358 & 0.03770 & 0.00089 \\
(40)-4 GDX & 0.51717 & 0.37478 & 0.18271 & 0.03739 & 0.00088 \\
Correlator & 0.51214 & 0.37012 & 0.17597 & 0.03398 & 0.00070 \\
\hline \# Symbols Tested & 36,000 & 42,200 & 66,200 & 100,000 & 100,000 \\
\hline
\end{tabular}

Table 4-24 SER Data for Figure 4-26

\section{Varying the amount of post-noise filtering}

A number of simulations were performed with the same types of networks with varying amounts of post-noise filtering performed. The first simulation shown in Figure 4-28 serves as a baseline with no post-noise filtering. 
a)

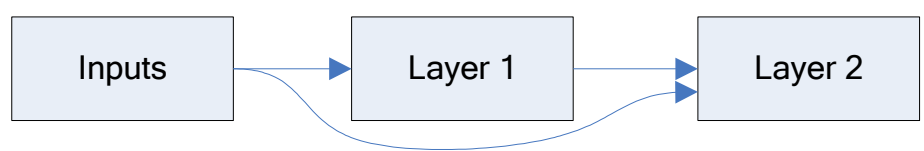

b)

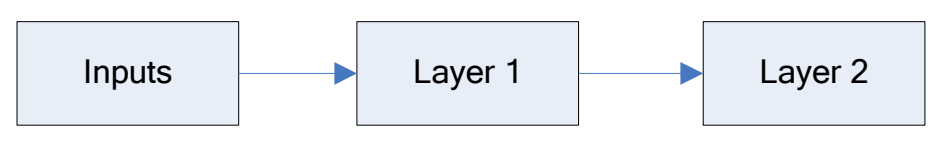

c)

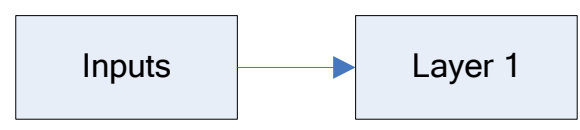

Figure 4-27 Layer connections for neural networks of Figure 4-28, Figure 4-29, Figure 4-30 and Figure 4-35. a) Connections for 2 layer cascade networks. b) Connections for 2 layer networks. c) Connections for single layer network.

\begin{tabular}{|c|c|c|c|c|c|}
\hline Parameter & Value & Value & Value & Value & Value \\
\hline Network Identifier & $\begin{array}{c}\text { (40)-16-4 } \\
\text { (sig,lin) casc }\end{array}$ & $\begin{array}{c}\text { (40)-16-4 } \\
\text { (sig,sig) casc }\end{array}$ & $\begin{array}{c}\text { (40)-16-4 } \\
\text { (sig.lin) }\end{array}$ & $\begin{array}{l}(40)-16-4 \\
\text { (sig,sig) }\end{array}$ & (40)-4 (lin) \\
\hline Number if Inputs & 40 & 40 & 40 & 40 & 40 \\
\hline Layer 1 Neurons & 16 & 16 & 16 & 16 & 16 \\
\hline $\begin{array}{l}\text { Layer } 1 \text { Transfer } \\
\text { Function }\end{array}$ & $S I G$ & $S I G$ & $S I G$ & $S I G$ & $S I G$ \\
\hline Layer 2 Neurons & 4 & 4 & 4 & 4 & - \\
\hline $\begin{array}{l}\text { Layer } 2 \text { Transfer } \\
\text { Function }\end{array}$ & LIN & $S I G$ & LIN & $S I G$ & - \\
\hline Training Function & $G D X$ & GDX & $G D X$ & $G D X$ & $G D X$ \\
\hline Initial Learning Rate & 0.05 & 0.05 & 0.05 & 0.05 & 0.05 \\
\hline Number of Networks & 3 & 3 & 3 & 3 & 3 \\
\hline Training Batch Size & 3,600 Symbols & 3,600 Symbols & 3,600 Symbols & 3,600 Symbols & 3,600 Symbols \\
\hline Training SNR(s) & $-11,-7,-3 \mathrm{~dB}$ & $-11,-7,-3 \mathrm{~dB}$ & $-11,-7,-3 \mathrm{~dB}$ & $-11,-7,-3 \mathrm{~dB}$ & $-11,-7,-3 \mathrm{~dB}$ \\
\hline $\begin{array}{l}\text { Training Epochs per } \\
\text { Training Session }\end{array}$ & 100 & 100 & 100 & 100 & 100 \\
\hline \# of Training Sessions & 2 & 2 & 2 & 2 & 2 \\
\hline $\begin{array}{l}\text { Post Noise Filter } \\
\text { Figure 4-28 }\end{array}$ & None & None & None & None & None \\
\hline $\begin{array}{l}\text { Post Noise Filter } \\
\text { Figure 4-29 }\end{array}$ & $\begin{array}{c}2^{\text {nd }} \text { order } 30 \mathrm{~Hz} \\
\text { with } \mathrm{Q}=0.5\end{array}$ & $\begin{array}{c}2^{\text {nd }} \text { order } 30 \mathrm{~Hz} \\
\text { with } \mathrm{Q}=0.5\end{array}$ & $\begin{array}{c}2^{\text {nd }} \text { order } 30 \mathrm{~Hz} \\
\text { with } \mathrm{Q}=0.5\end{array}$ & $\begin{array}{c}2^{\text {nd }} \text { order } 30 \mathrm{~Hz} \\
\text { with } \mathrm{Q}=0.5\end{array}$ & $\begin{array}{c}2^{\text {nd }} \text { order } 30 \mathrm{~Hz} \\
\text { with } Q=0.5\end{array}$ \\
\hline $\begin{array}{l}\text { Post Noise Filter } \\
\text { Figure 4-30 }\end{array}$ & $\begin{array}{c}2^{\text {nd }} \text { order } 20 \mathrm{~Hz} \\
\text { with } \mathrm{Q}=0.5\end{array}$ & $\begin{array}{c}2^{\text {nd }} \text { order } 20 \mathrm{~Hz} \\
\text { with } \mathrm{Q}=0.5\end{array}$ & $\begin{array}{c}2^{\text {nd }} \text { order } 20 \mathrm{~Hz} \\
\text { with } \mathrm{Q}=0.5\end{array}$ & $\begin{array}{c}2^{\text {nd }} \text { order } 20 \mathrm{~Hz} \\
\text { with } \mathrm{Q}=0.5\end{array}$ & $\begin{array}{c}2^{\text {nd }} \text { order } 20 \mathrm{~Hz} \\
\text { with } \mathrm{Q}=0.5\end{array}$ \\
\hline $\begin{array}{l}\text { Post Noise Filter } \\
\text { Figure 4-31 }\end{array}$ & $\begin{array}{c}2^{\text {nd }} \text { order } 10 \mathrm{~Hz} \\
\text { with } \mathrm{Q}=0.5\end{array}$ & $\begin{array}{c}2^{\text {nd }} \text { order } 10 \mathrm{~Hz} \\
\text { with } \mathrm{Q}=0.5\end{array}$ & $\begin{array}{c}2^{\text {nd }} \text { order } 10 \mathrm{~Hz} \\
\text { with } \mathrm{Q}=0.5\end{array}$ & $\begin{array}{c}2^{\text {nd }} \text { order } 10 \mathrm{~Hz} \\
\text { with } \mathrm{Q}=0.5\end{array}$ & $\begin{array}{c}2^{\text {nd }} \text { order } 10 \mathrm{~Hz} \\
\text { with } \mathrm{Q}=0.5\end{array}$ \\
\hline
\end{tabular}

Table 4-25 Training and Test Parameters for Figure 4-28, Figure 4-29, Figure 4-33, and Figure 4-35 


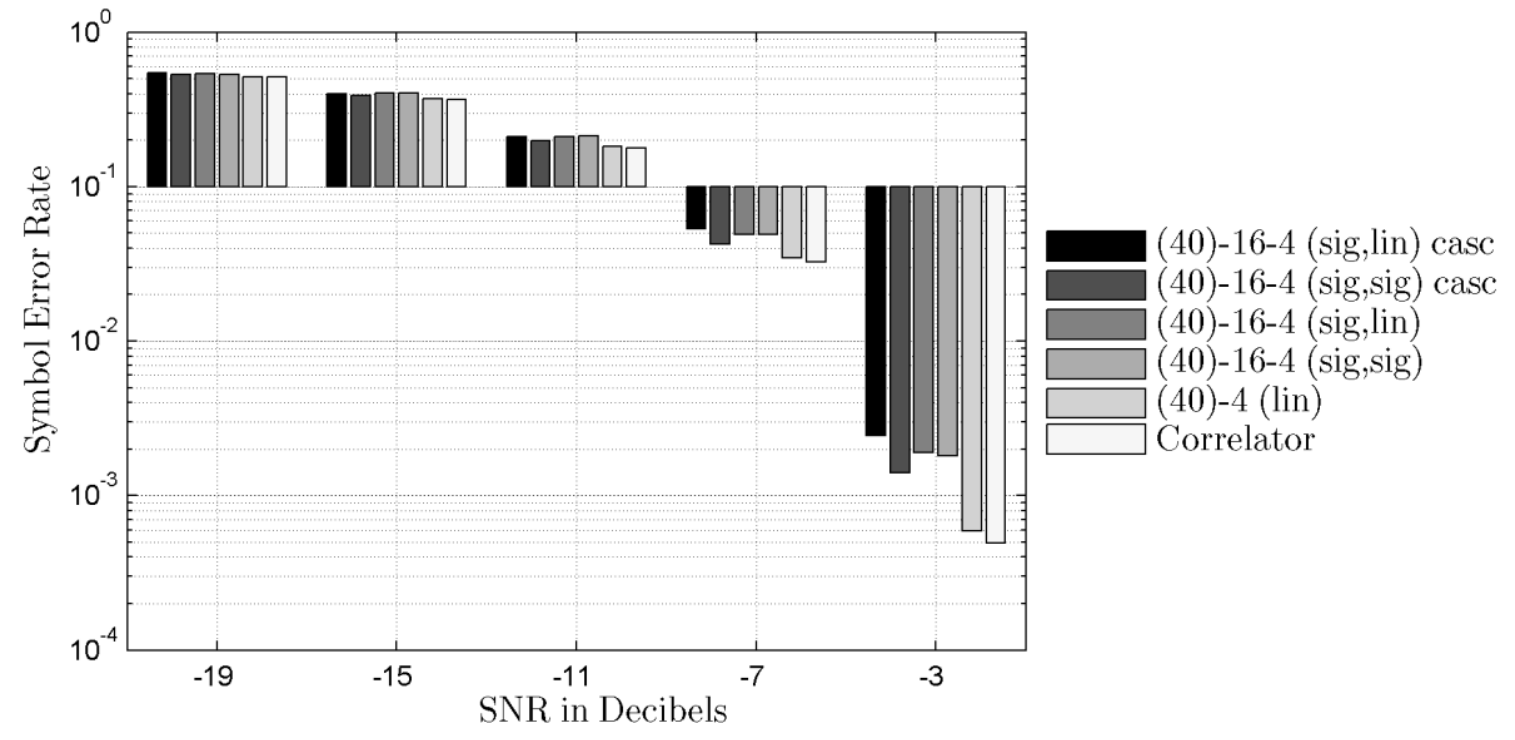

Figure 4-28 No Post-Noise Filtering for AWGN with 4 symbols

\begin{tabular}{|ccccrr|}
\hline \multicolumn{7}{c}{ SNR } \\
\hline Receiver & -19dB & -15dB & -11dB & -7dB & -3dB \\
\hline (40)-16-4 (sig,lin) casc & 0.54168 & 0.39606 & 0.21115 & 0.05342 & 0.00245 \\
$(40)-16-4$ (sig,sig) casc & 0.52956 & 0.38725 & 0.19754 & 0.04262 & 0.00140 \\
(40)-16-4 (sig,lin) & 0.53881 & 0.40110 & 0.20947 & 0.04888 & 0.00189 \\
(40)-16-4 (sig,sig) & 0.53445 & 0.40109 & 0.21292 & 0.04872 & 0.00181 \\
(40)-4 (lin) & 0.51253 & 0.36932 & 0.18150 & 0.03468 & 0.00059 \\
Correlator & 0.51042 & 0.36491 & 0.17772 & 0.03247 & 0.00049 \\
\hline \# Symbols Tested & 36,000 & 42,200 & 64,800 & 100,000 & 100,000 \\
\hline
\end{tabular}

The correlator and the single layer linear network are clearly the best performers under these conditions. 


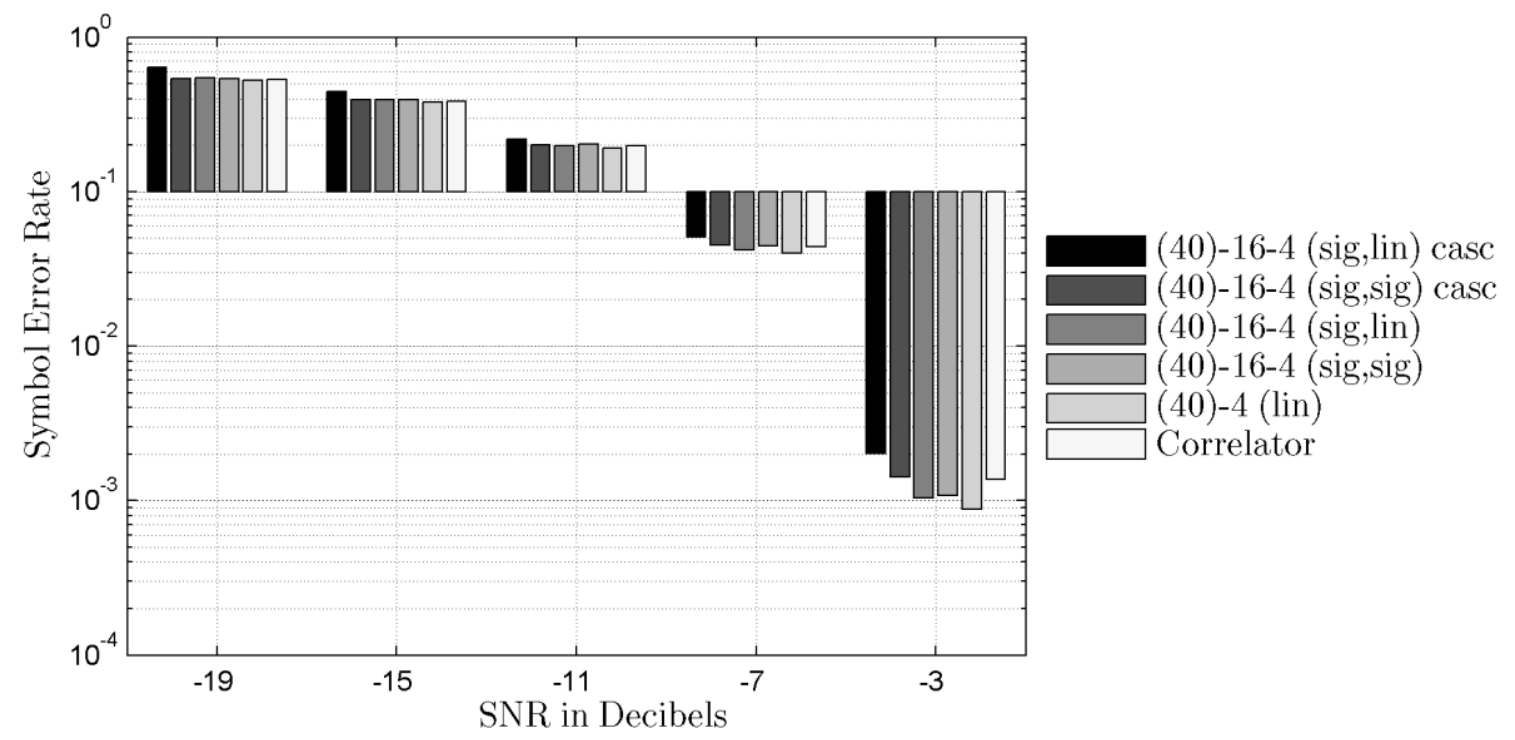

Figure 4-29 30Hz $2^{\text {nd }}$ Order Low Pass Post-Noise Filtering for AWGN with 4 symbols

\begin{tabular}{|cccccc|}
\hline \multicolumn{7}{c}{ SNR } \\
\hline Receiver & $\mathbf{- 1 9 d B}$ & $\mathbf{- 1 5 d B}$ & $\mathbf{- 1 1 d B}$ & $\mathbf{- 7 d B}$ & -3dB \\
\hline (40)-16-4 (sig,lin) casc & 0.63500 & 0.44363 & 0.21761 & 0.05080 & 0.00201 \\
$(40)-16-4$ (sig,sig) casc & 0.53970 & 0.39481 & 0.20154 & 0.04487 & 0.00142 \\
(40)-16-4 (sig,lin) & 0.54447 & 0.39403 & 0.19715 & 0.04194 & 0.00104 \\
(40)-16-4 (sig,sig) & 0.53692 & 0.39462 & 0.20310 & 0.04437 & 0.00108 \\
(40)-4 (lin) & 0.52500 & 0.38107 & 0.19113 & 0.03997 & 0.00088 \\
Correlator & 0.52980 & 0.38514 & 0.19744 & 0.04413 & 0.00137 \\
\hline \# Symbols Tested & 35,600 & 41,400 & 62,600 & 100,000 & 100,000 \\
\hline
\end{tabular}

Table 4-26 SER Data for Figure 4-29

Figure 4-29 shows a slight decrease in the performance for the correlator and single layer linear network when a $30 \mathrm{~Hz}$ post-noise filter is used. Decreasing the filter corner to $20 \mathrm{~Hz}$ results in a minimal change in performance as can be seen in Figure 4-30. 


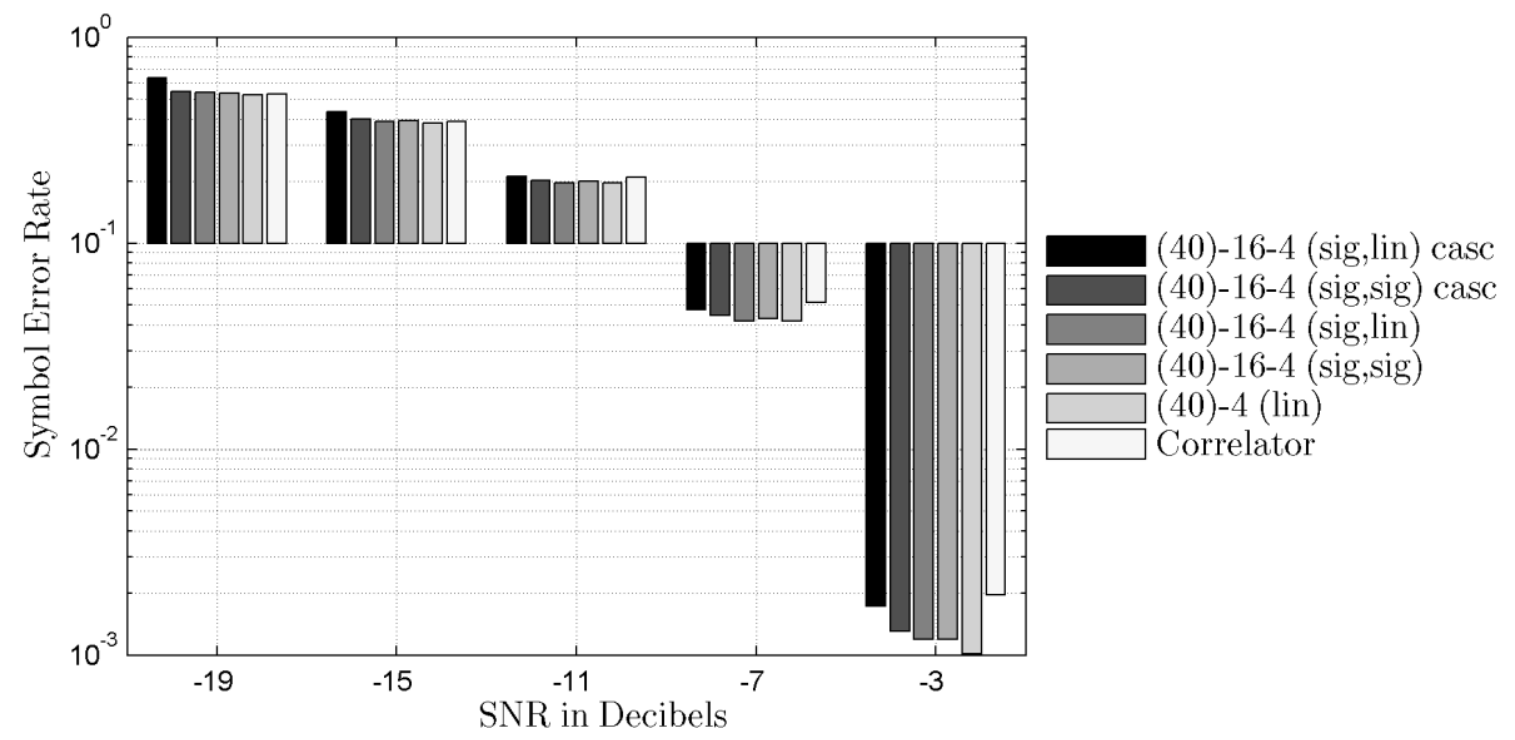

Figure 4-30 $20 \mathrm{~Hz}$ 2nd Order Low Pass Post-Noise Filtering for AWGN with 4 symbols

\begin{tabular}{|cccccc|}
\hline \multicolumn{7}{c}{ SNR } \\
\hline Receiver & -19dB & -15dB & \multicolumn{1}{c|}{-11dB } & \multicolumn{1}{c|}{-7dB } & -3dB \\
\hline (40)-16-4 (sig,lin) casc & 0.63169 & 0.43246 & 0.21038 & 0.04739 & 0.00173 \\
(40)-16-4 (sig,sig) casc & 0.54411 & 0.39820 & 0.20199 & 0.04449 & 0.00131 \\
(40)-16-4 (sig,lin) & 0.53779 & 0.39067 & 0.19566 & 0.04185 & 0.00119 \\
(40)-16-4 (sig,sig) & 0.53444 & 0.39192 & 0.19875 & 0.04295 & 0.00120 \\
(40)-4 (lin) & 0.52456 & 0.38276 & 0.19568 & 0.04199 & 0.00101 \\
Correlator & 0.53145 & 0.39063 & 0.20789 & 0.05136 & 0.00196 \\
\hline Symbols Simulated & 35,800 & 41,600 & 62,200 & 100,000 & 100,000 \\
\hline
\end{tabular}

Table 4-27 SER Data for Figure 4-30

The simulation shown in Figure 4-31 used a $10 \mathrm{~Hz}$ filter. The performance of the correlator decreased significantly, whereas the neural network receivers only saw a slight decrease. 


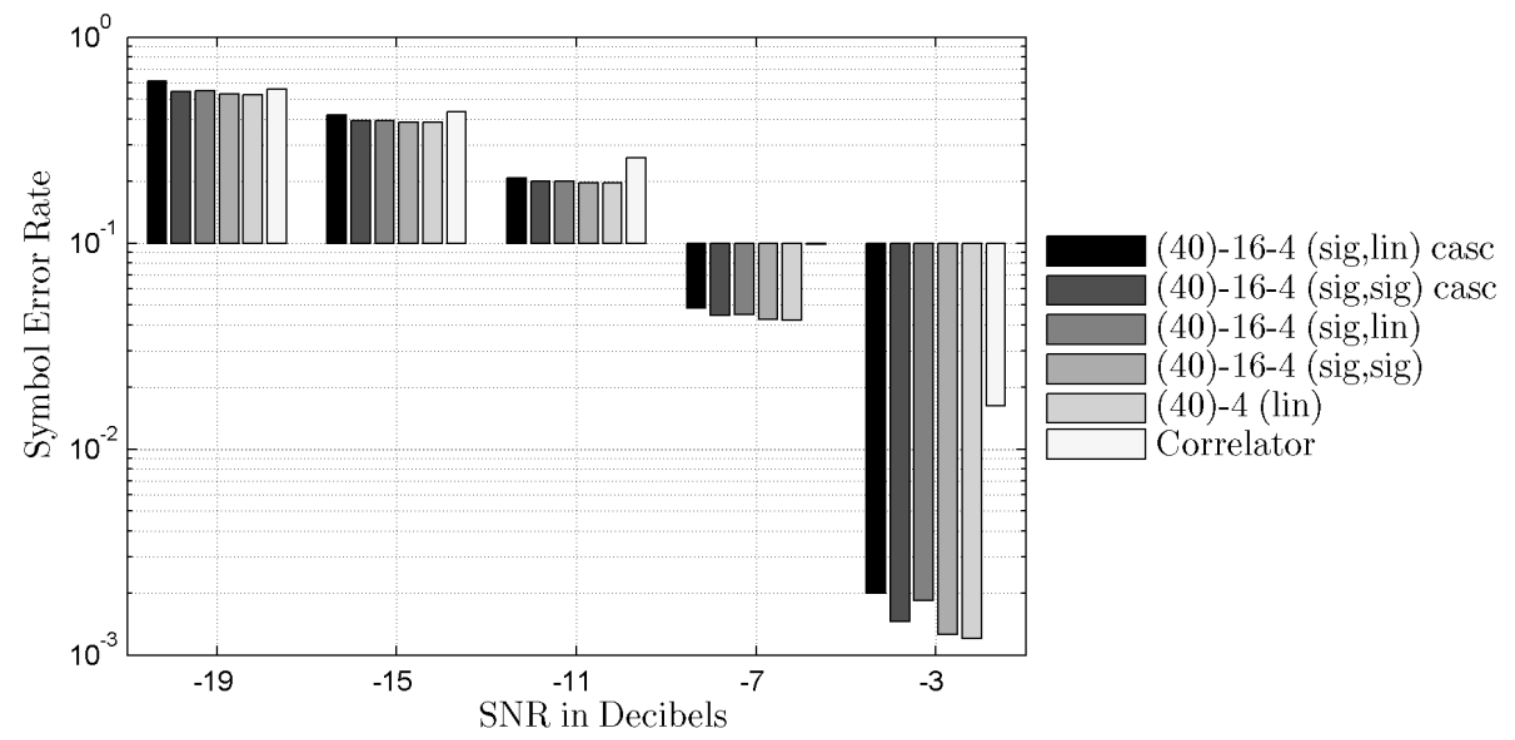

Figure 4-31 $10 \mathrm{~Hz}$ 2nd Order Low Pass Post-Noise Filtering for AWGN with 4 symbols

SNR

\begin{tabular}{|cccccc|}
\hline Receiver & $\mathbf{- 1 9 d B}$ & $\mathbf{- 1 5 d B}$ & $\mathbf{- 1 1 d B}$ & \multicolumn{1}{c|}{$\mathbf{d B}$} & -3dB \\
\hline (40)-16-4 (sig,lin) casc & 0.61269 & 0.41933 & 0.20685 & 0.04820 & 0.00199 \\
$(40)-16-4$ (sig,sig) casc & 0.54301 & 0.39278 & 0.20039 & 0.04444 & 0.00145 \\
(40)-16-4 (sig,lin) & 0.54772 & 0.39435 & 0.19914 & 0.04503 & 0.00185 \\
(40)-16-4 (sig,sig) & 0.53116 & 0.38709 & 0.19654 & 0.04261 & 0.00126 \\
(40)-4 (lin) & 0.52545 & 0.38524 & 0.19594 & 0.04232 & 0.00121 \\
Correlator & 0.55709 & 0.43362 & 0.25971 & 0.09837 & 0.01615 \\
\hline Symbols Simulated & 35,800 & 41,400 & 62,000 & 100,000 & 100,000 \\
\hline
\end{tabular}

Table 4-28 SER Data for Figure 4-31 


\subsection{Simulations Using AWGN and 8 Symbols}

\subsubsection{Varying Input Size}

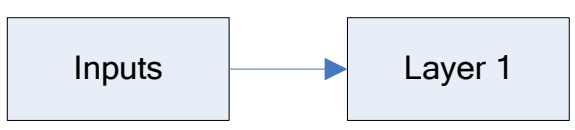

Figure 4-32 Layer connections for neural networks of Figure 4-33

\begin{tabular}{|c|c|c|c|c|}
\hline Parameter & Value & Value & Value & Value \\
\hline Network Identifier & $(80)-8$ & $(40)-8$ & $(16)-8$ & $(8)-8$ \\
\hline Number if Inputs & 80 & 40 & 16 & 8 \\
\hline Layer 1 Neurons & 8 & 8 & 8 & 8 \\
\hline Layer 1 Transfer Function & $L I N$ & $L I N$ & $L I N$ & LIN \\
\hline Training Function & $G D X$ & $G D X$ & $G D X$ & $G D X$ \\
\hline Initial Learning Rate & 0.05 & 0.05 & 0.05 & 0.05 \\
\hline Number of Networks & 3 & 3 & 3 & 3 \\
\hline Training Batch Size & 4,800 Symbols & 4,800 Symbols & 4,800 Symbols & 4,800 Symbols \\
\hline Training SNR(s) & $-11,-7,-3 \mathrm{~dB}$ & $-11,-7,-3 \mathrm{~dB}$ & $-11,-7,-3 \mathrm{~dB}$ & $-11,-7,-3 \mathrm{~dB}$ \\
\hline Training Epochs per & 150 & 150 & 150 & 150 \\
\hline Training Session & 2 & 2 & 2 & 2 \\
\hline \# of Training Sessions & \multicolumn{2}{|c|}{150} \\
\hline
\end{tabular}

Table 4-29 Training and test parameters for Figure 4-33 


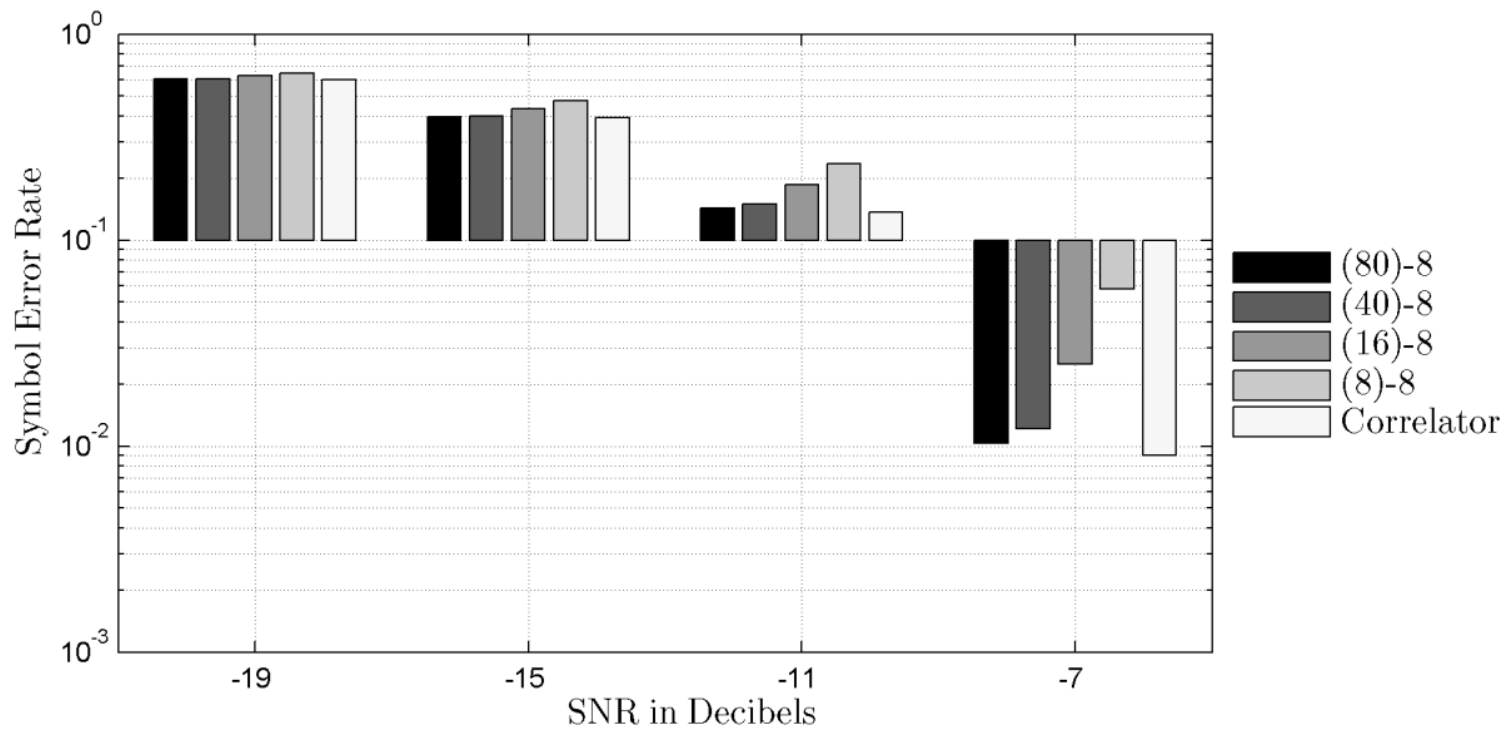

Figure 4-33 Varying input size of a single layer linear network for AWGN with 8 symbols

\begin{tabular}{|ccrrr}
\multicolumn{5}{c}{ SNR } \\
\hline Receiver & -19dB & $\mathbf{- 1 5 d B}$ & $\mathbf{- 1 1 d B}$ & -7dB \\
\hline$(80)-8$ (lin) & 0.60652 & 0.39585 & 0.14333 & 0.01029 \\
$(40)-8($ lin) & 0.60759 & 0.40111 & 0.14891 & 0.01219 \\
$(16)-8$ (lin) & 0.62773 & 0.43511 & 0.18516 & 0.02494 \\
$(8)-8$ (lin) & 0.64816 & 0.47470 & 0.23440 & 0.05787 \\
Correlator & 0.60056 & 0.39133 & 0.13621 & 0.00900 \\
\hline \# Symbols Tested & 30,400 & 36,800 & 75,200 & 94,400 \\
\hline
\end{tabular}

Table 4-30 SER Data for Figure 4-33

The relative performance of the networks is similar to the tests performed with 4symbol set, with the larger input sizes having lower symbol error rates. Figure 4-35 shows similar results for a 2-layer network using the SIG transfer function in each layer. The performance of the linear single layer network with 80 inputs performs the best. 


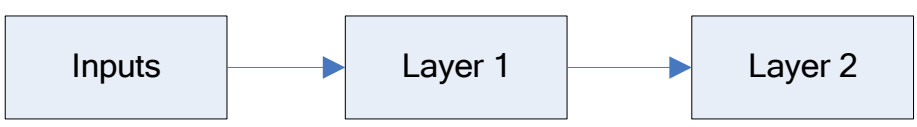

Figure 4-34 Layer connections for neural networks of Figure 4-35

\begin{tabular}{|c|c|c|c|c|}
\hline Parameter & Value & Value & Value & Value \\
\hline Network Identifier & $(80)-16-8$ & $(40)-16-8$ & $(16)-16-8$ & $(8)-16-8$ \\
\hline Number if Inputs & 80 & 40 & 16 & 8 \\
\hline Layer 1 Neurons & 16 & 16 & 16 & 16 \\
\hline Layer 1 Transfer Function & $S I G$ & $S I G$ & $S I G$ & $S I G$ \\
\hline Layer 2 Neurons & 8 & 8 & 8 & 8 \\
\hline Layer 2 Transfer Function & $S I G$ & $S I G$ & $S I G$ & $S I G$ \\
\hline Training Function & $G D X$ & $G D X$ & $G D X$ & $G D X$ \\
\hline Initial Learning Rate & 0.05 & 0.05 & 0.05 & 0.05 \\
\hline Number of Networks & 5 & 5 & 5 & 5 \\
\hline Training Batch Size & 4,800 Symbols & 4,800 Symbols & 4,800 Symbols & 4,800 Symbols \\
\hline Training SNR(s) & $-11,-7,-3 \mathrm{~dB}$ & $-11,-7,-3 \mathrm{~dB}$ & $-11,-7,-3 \mathrm{~dB}$ & $-11,-7,-3 \mathrm{~dB}$ \\
\hline Training Epochs per & 150 & 150 & 150 & 150 \\
\hline Training Session & 2 & 2 & 2 & 2 \\
\hline \# of Training Sessions & 2 & &
\end{tabular}

Table 4-31 Training and test parameters for Figure 4-35

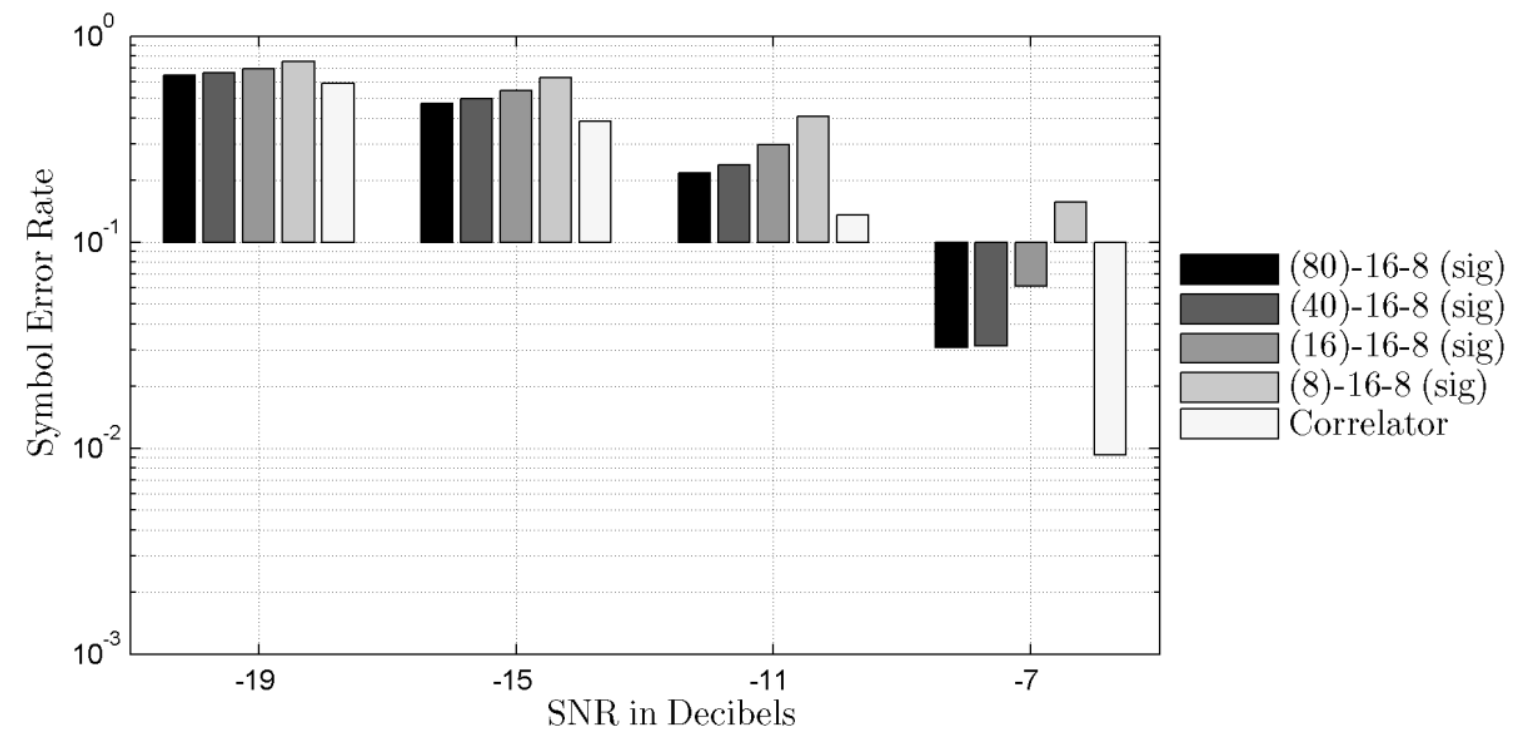

Figure 4-35 Varying Input Size of a 2-Layer Non-Linear Network for AWGN with 8 symbols 
SNR

\begin{tabular}{crrrr}
\hline Receiver & $\mathbf{- 1 9 d B}$ & \multicolumn{1}{c}{$\mathbf{- 1 5 d B}$} & \multicolumn{1}{c}{$\mathbf{- 1 1 d B}$} & \multicolumn{1}{c}{$\mathbf{- 7 d B}$} \\
\hline$(80)-16-8(\mathrm{sig})$ & 0.64608 & 0.47197 & 0.21706 & 0.03076 \\
$(40)-16-8(\mathrm{sig})$ & 0.66368 & 0.49783 & 0.23626 & 0.03133 \\
$(16)-16-8(\mathrm{sig})$ & 0.69303 & 0.54404 & 0.29580 & 0.06101 \\
$(8)-16-8(\mathrm{sig})$ & 0.75147 & 0.62740 & 0.40615 & 0.15607 \\
Correlator & 0.58760 & 0.38546 & 0.13477 & 0.00924 \\
\hline \# Symbols Tested & 30,400 & 36,800 & 75,200 & 94,400 \\
\hline
\end{tabular}

Table 4-32 SER Data for Figure 4-35

\subsubsection{Cascade Networks}

The simulations of Figure 4-37 involve cascade networks of different sizes. The last layer in each network utilizes the LIN transfer function, whereas all the other layers use SIG.

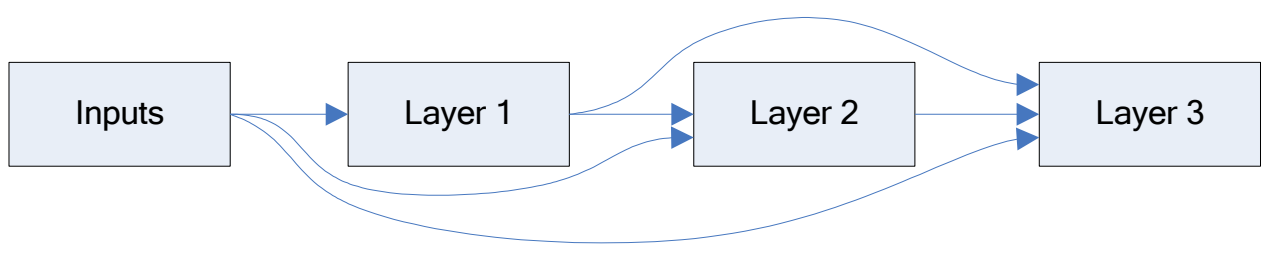

Figure 4-36 Layer connections for neural networks of Figure 4-37 


\begin{tabular}{|c|c|c|c|}
\hline Parameter & Value & Value & Value \\
\hline Network Identifier & $(80)-8-8-8$ casc & $(80)-4-4-8$ casc & $(80)-0-4-8$ casc \\
\hline Number if Inputs & 80 & 80 & 80 \\
\hline Layer 1 Neurons & 8 & 4 & 0 \\
\hline Layer 1 Transfer Function & $S I G$ & $S I G$ & - \\
\hline Layer 2 Neurons & 8 & 4 & 4 \\
\hline Layer 2 Transfer Function & $S I G$ & $S I G$ & 8 \\
\hline Layer 3 Neurons & 8 & 8 & $L I N$ \\
\hline Layer 3 Transfer Function & $L I N$ & $L I N$ & $G D X$ \\
\hline Training Function & $G D X$ & $G D X$ & 0.05 \\
\hline Initial Learning Rate & 0.05 & 0.05 & 5 \\
\hline Number of Networks & 5 & 5 & 4,800 Symbols \\
\hline Training Batch Size & 4,800 Symbols & 4,800 Symbols & $-11,-7,-3 \mathrm{~dB}$ \\
\hline Training SNR(s) & $-11,-7,-3 \mathrm{~dB}$ & $-11,-7,-3 \mathrm{~dB}$ & 150 \\
\hline Training Epochs per Training & 150 & 150 & 2 \\
\hline Session & 2 & 2 & \\
\hline \# of Training Sessions & 150 &
\end{tabular}

Table 4-33 Training and test parameters for Figure 4-37

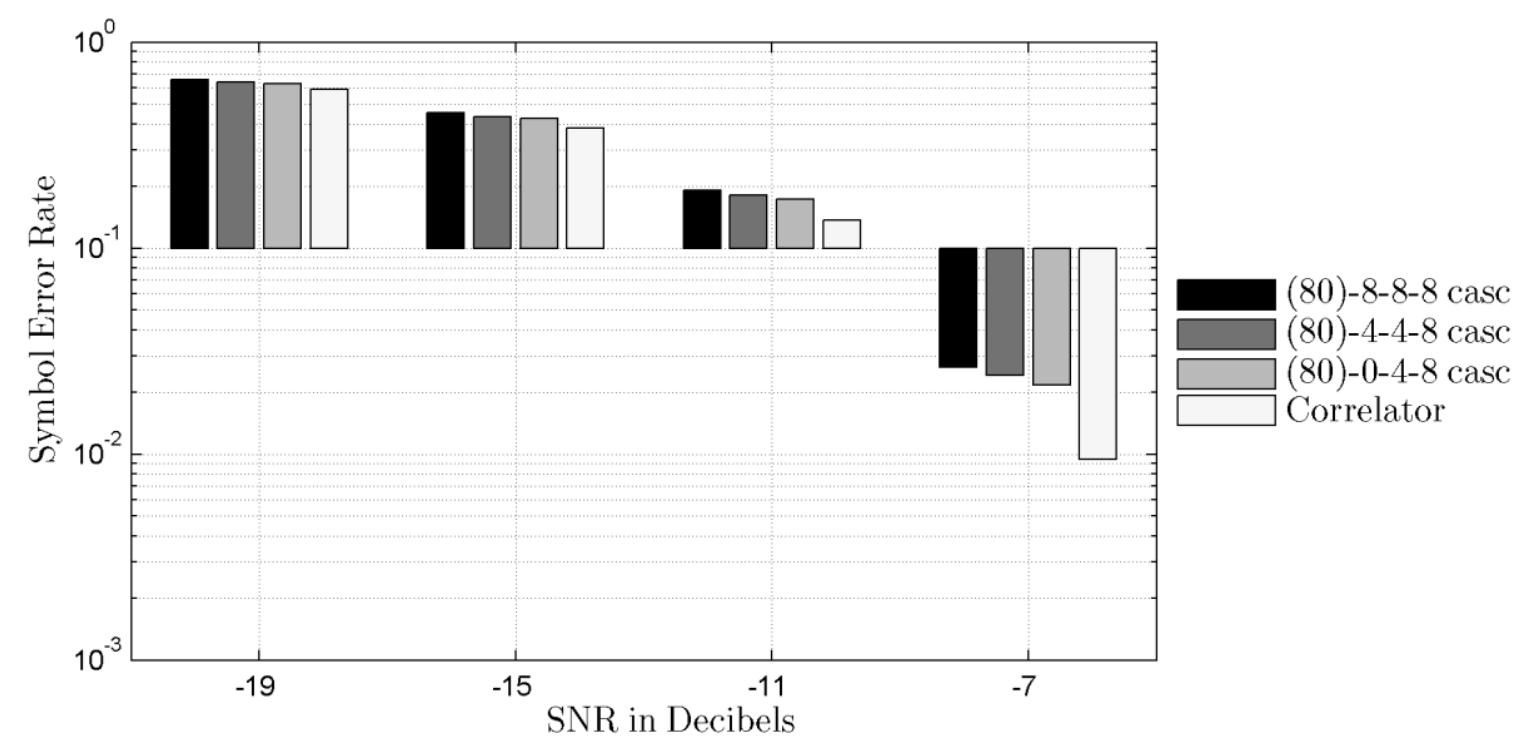

Figure 4-37 Cascade Networks for AWGN with 8 symbols 


\begin{tabular}{|crrrr}
\multicolumn{5}{c}{ SNR } \\
\hline Receiver & $\mathbf{- 1 9 d B}$ & $\mathbf{- 1 5 d B}$ & $\mathbf{- 1 1 d B}$ & -7dB \\
\hline$(80)-8-8-8$ casc & 0.65754 & 0.45229 & 0.19110 & 0.02640 \\
$(80)-4-4-8$ casc & 0.63940 & 0.43420 & 0.18120 & 0.02411 \\
(80)-0-4-8 casc & 0.63042 & 0.42717 & 0.17309 & 0.02163 \\
Correlator & 0.59117 & 0.38398 & 0.13605 & 0.00943 \\
\hline \# Symbols Tested & 30,400 & 36,800 & 75,200 & 94,400 \\
\hline
\end{tabular}

Table 4-34 SER Data for Figure 4-37

There is no significant performance difference between the various cascade networks tested. The cascade networks did outperform the 2-layer non-linear networks, but the single layer linear networks still performed the best. Further simulations with the 8 -symbol code set and AWGN did not result in any change in performance when compared with the 4-symbol set, and thus were not included in this paper.

\subsection{Simulations Using Non-AWGN and 4 Symbols}

Frequency based methods were used to generated noise with varying PSD shapes. The same definition of SNR as used with the AWGN simulations was used in order to allow for a better insight into the effect of slight variations on the PSD shape. The spectral density per unit bandwidth of the first set of noise shapes is proportional to $\mathrm{f}^{(-\beta)}$. When $\beta$ is equal to 0 this is the same thing as AWGN. Using a $\beta$ of 1 results in pink noise, whereas Brownian noise is generated by setting $\beta$ to 2 . Other similar PSD shapes were generated via the frequency domain to have the magnitude of the PSD decrease as the distance from a set frequency increased. See Equation 3-22 for the exact method of generating the PSD shape for this nonwhite noise. The next few figures show the power spectral densities of the noise sources used. 


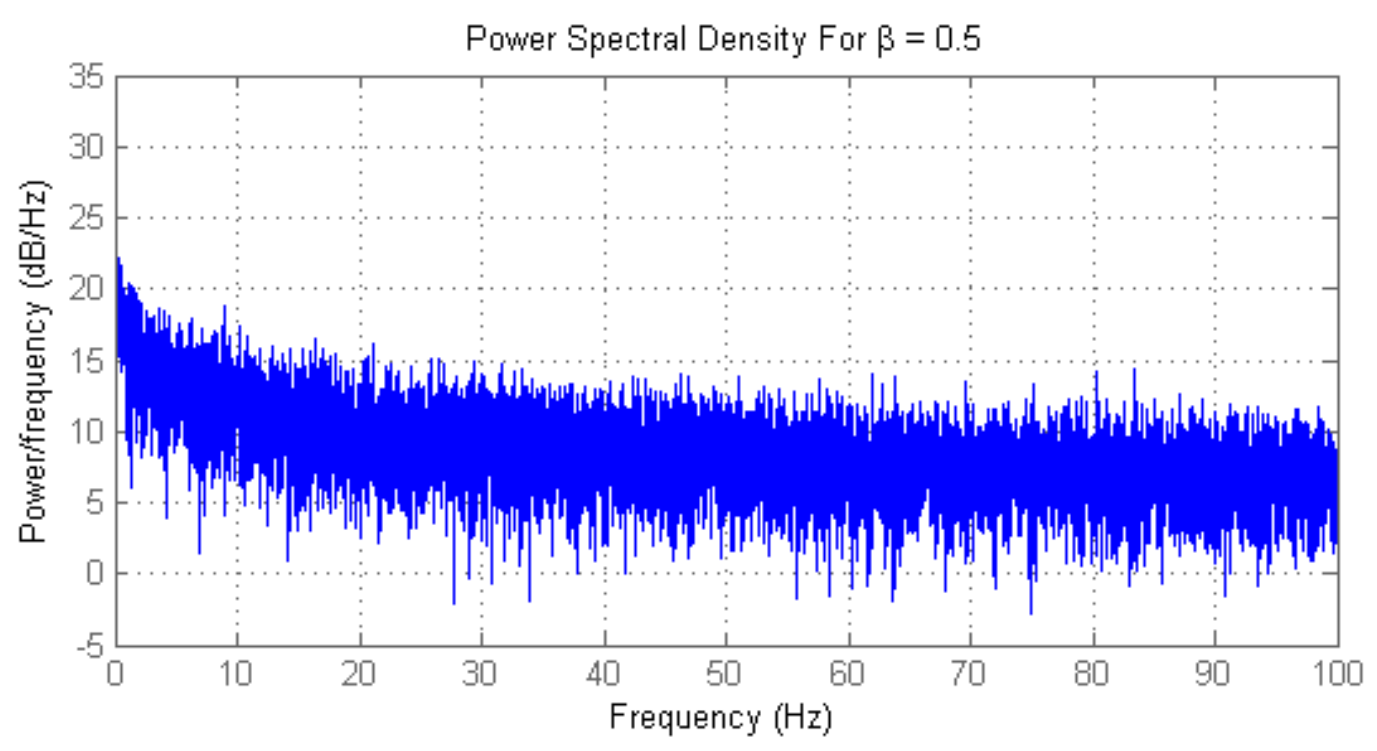

Figure 4-38 PSD for $\beta=0.5$

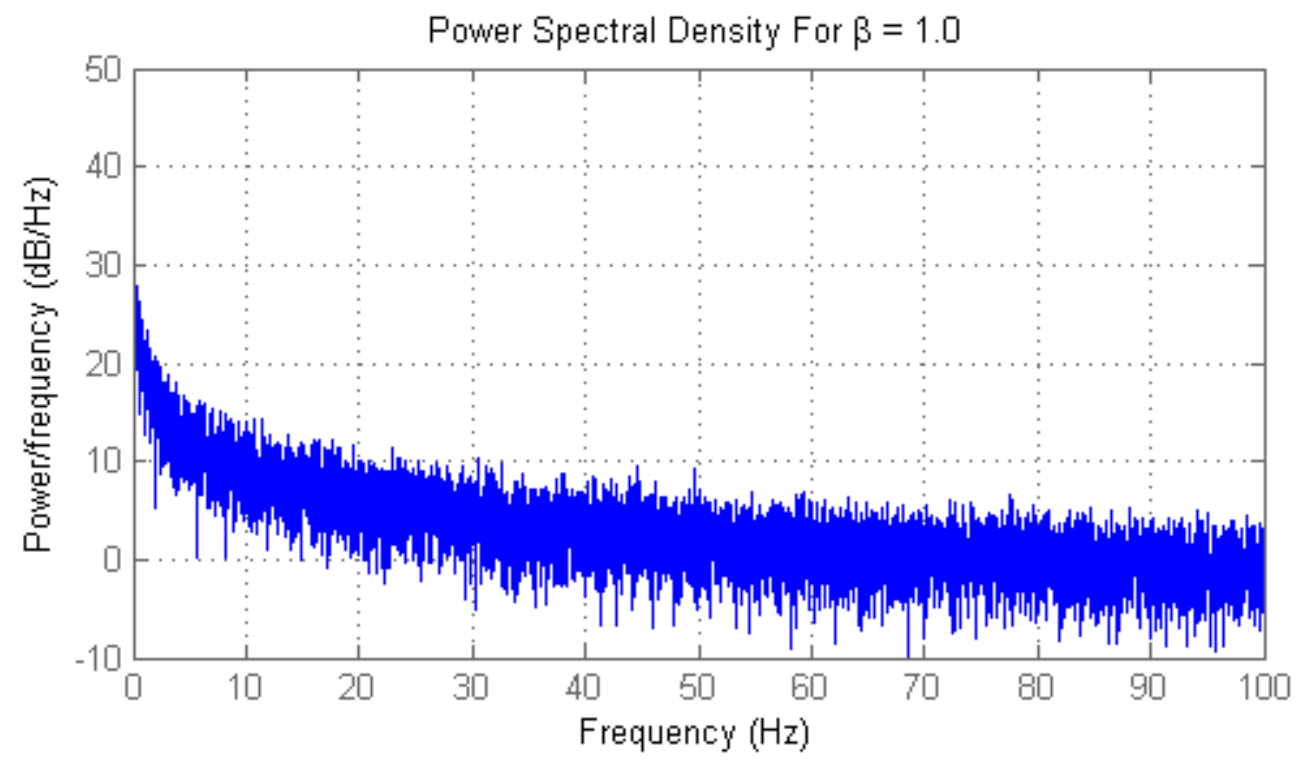

Figure 4-39 PSD for $\beta=1.0$ (1/f or Pink Noise) 


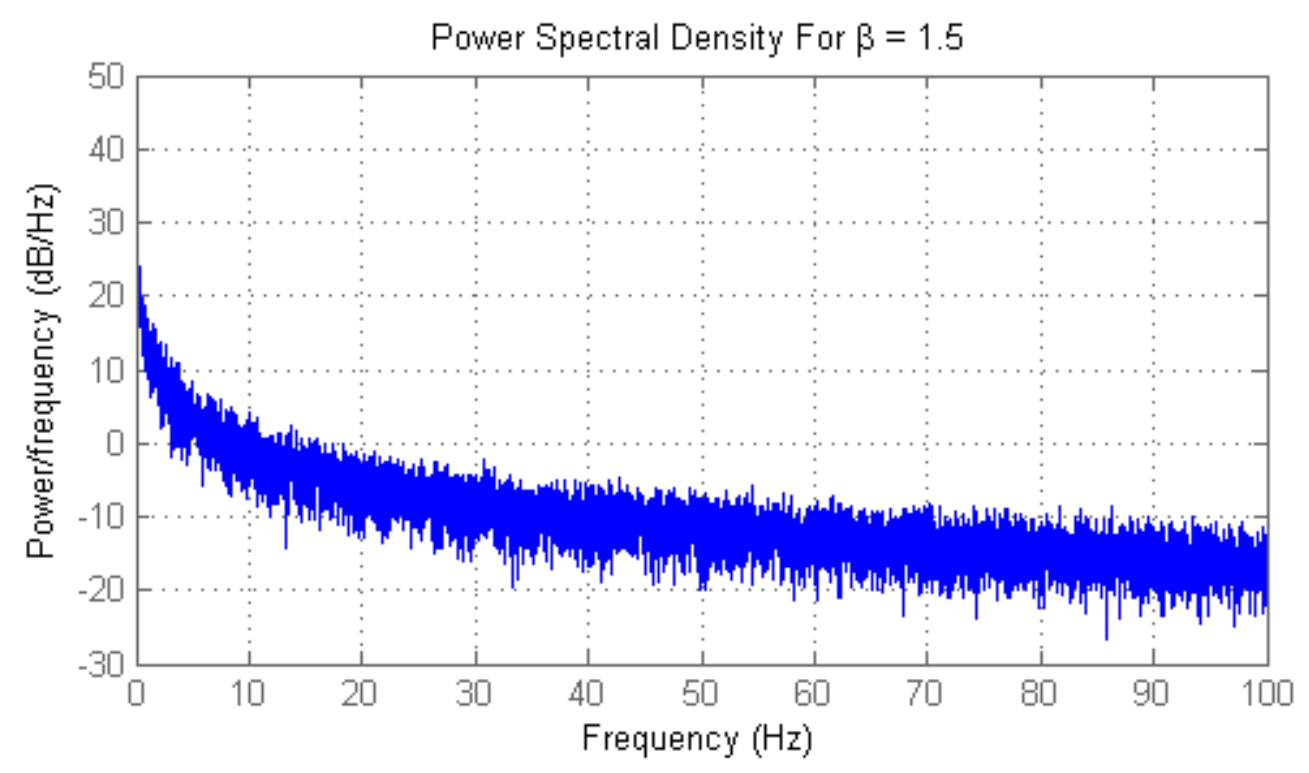

Figure 4-40 PSD for $\beta=1.5$

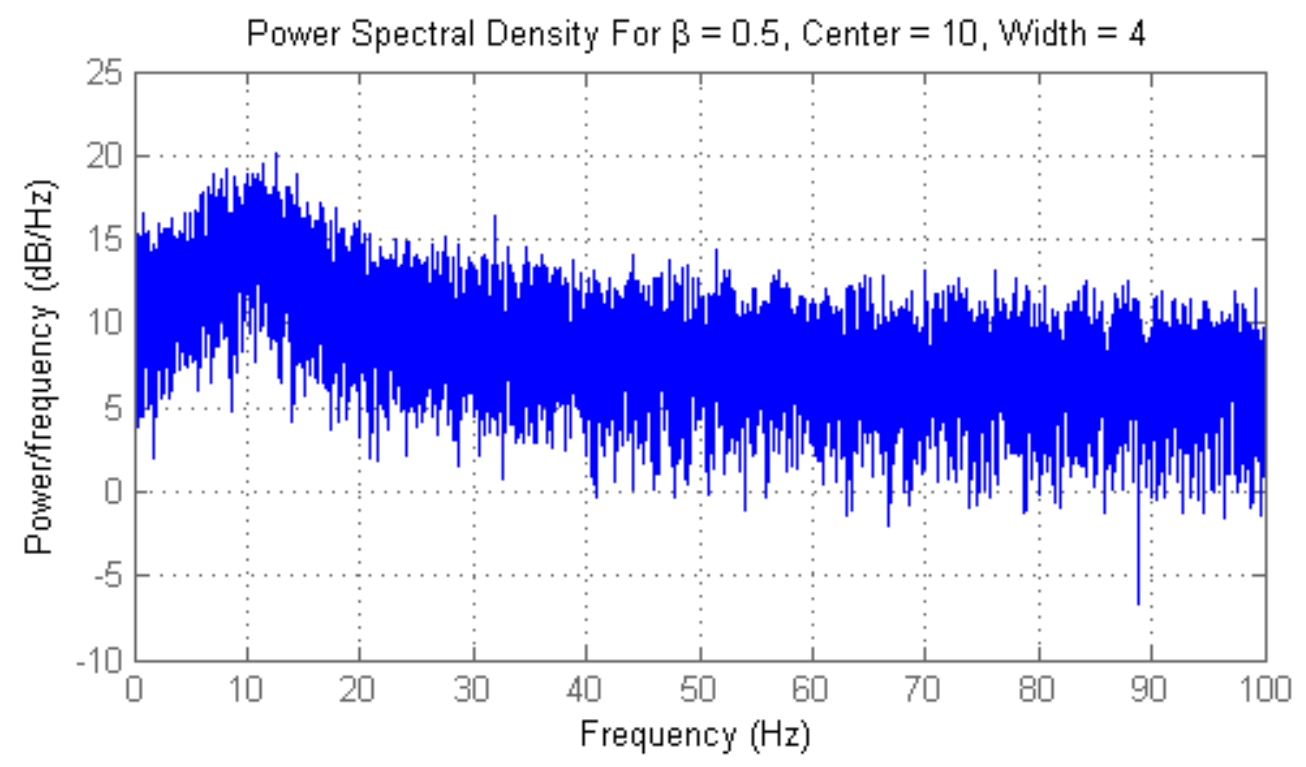

Figure 4-41 PSD For $\beta=0.5$ Centered at $10 \mathrm{~Hz}$ With a $4 \mathrm{~Hz}$ Plateau Width 


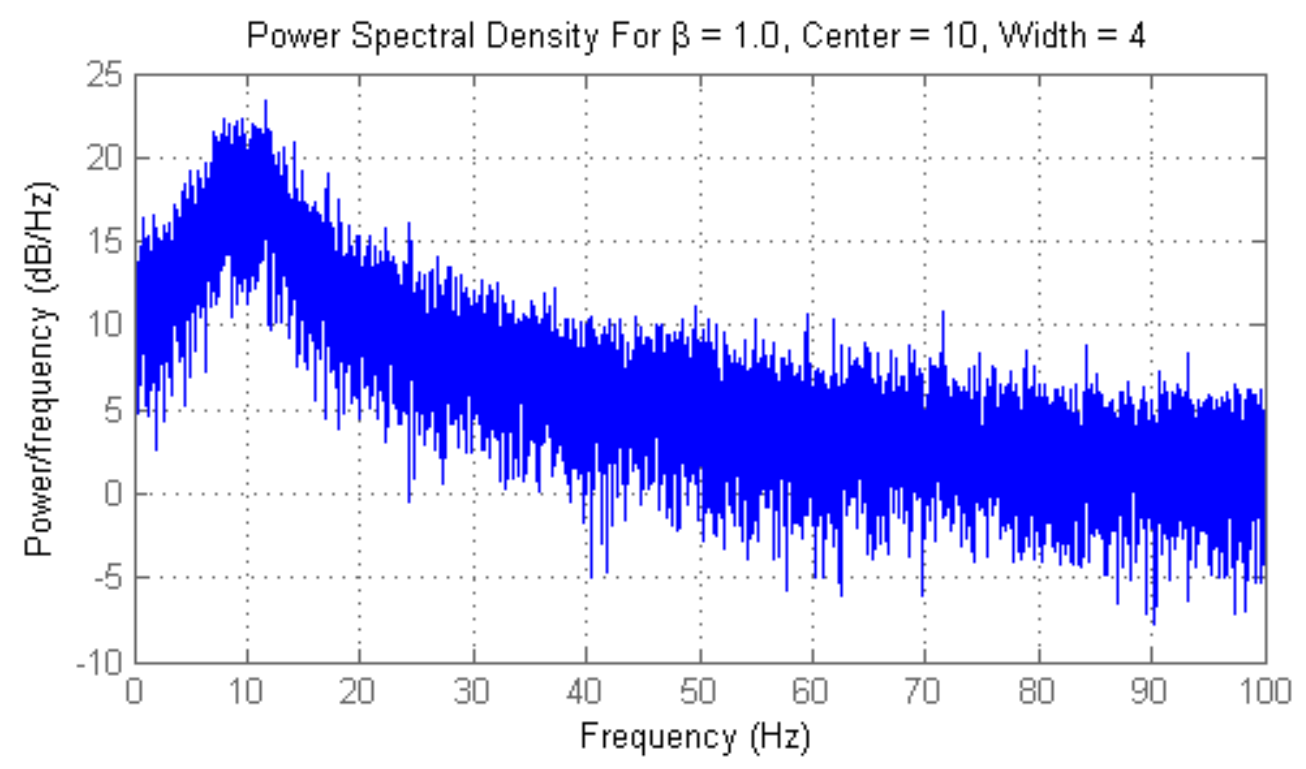

Figure 4-42 PSD For $\beta=1.0$ Centered at $10 \mathrm{~Hz}$ With a $4 \mathrm{~Hz}$ Plateau Width

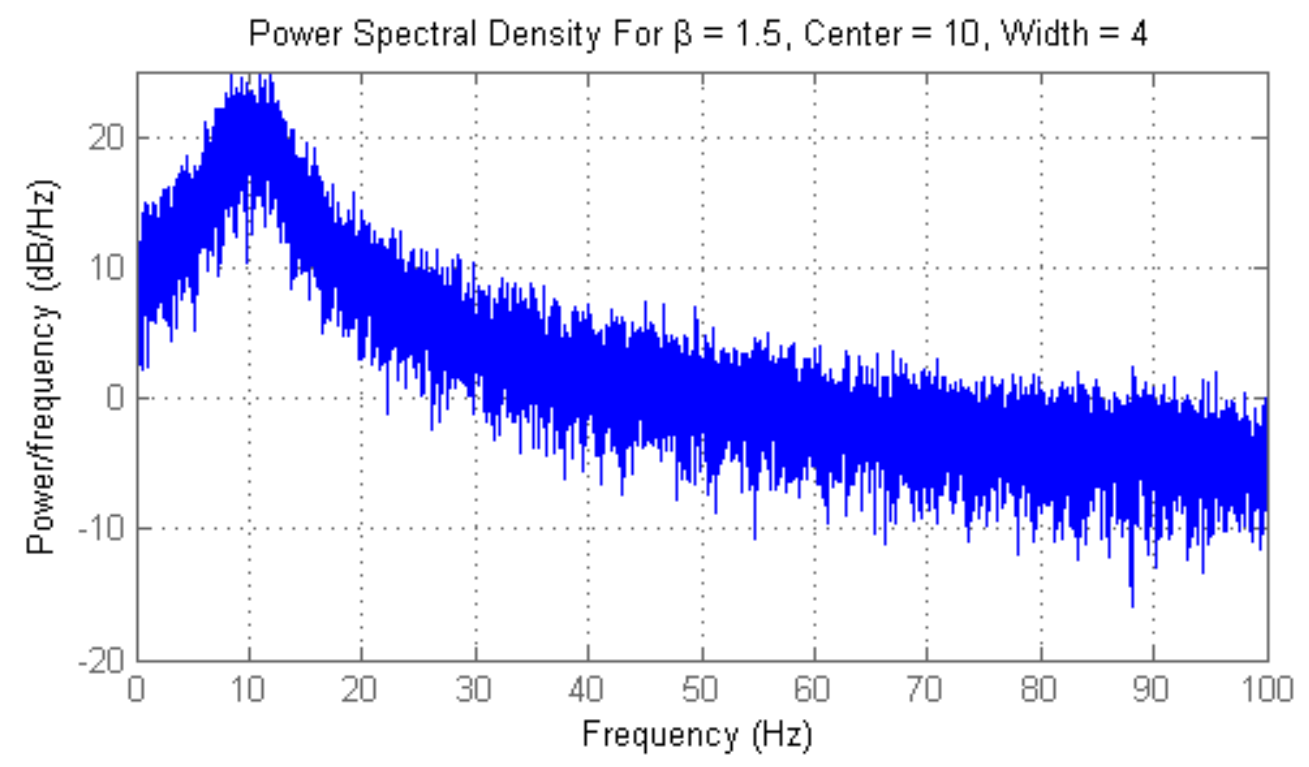

Figure 4-43 PSD For $\beta=1.5$ Centered at $10 \mathrm{~Hz}$ With a $4 \mathrm{~Hz}$ Plateau Width 


\subsubsection{Varying Training Algorithm}

Figure 4-45 shows the results of simulating single layer linear and non-linear networks with pink noise. The training algorithms used were Levenberg Marquardt (LM), conjugant gradient (CGP), and gradient descent with momentum and adaptive learning rate (GDX). The networks using the SIG transfer function outperformed those with a purely linear transfer function. Training with the Levenberg Marquardt algorithm resulted in the best performance for the $0 \mathrm{~dB}$ and $4 \mathrm{~dB}$ SNR cases. Training with the gradient descent with momentum and adaptive learning rate algorithm resulted in better performance than either the conjugate gradient method or the Levenberg Marquardt algorithm for the 8dB SNR case. Note that the Levenberg Marquardt training function used a reduced number of training epochs due to its ability to converge more quickly, which can lead to over fitting to the training data. The performance of the simple correlation receiver is considerably worse than any of the network receivers tested.

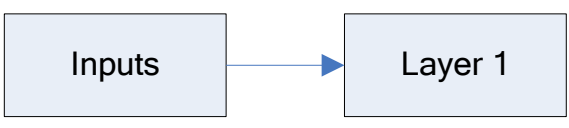

Figure 4-44 Layer connections for neural networks of Figure 4-45 


\begin{tabular}{|c|c|c|c|c|c|c|}
\hline Parameter & Value & Value & Value & Value & Value & Value \\
\hline Network Identifier & $\begin{array}{c}\text { (40)-4 (lin) } \\
\text { CGP }\end{array}$ & $\begin{array}{l}\text { (40)-4 (lin) } \\
\text { LM }\end{array}$ & $\begin{array}{l}\text { (40)-4 (lin) } \\
\text { GDX }\end{array}$ & $\begin{array}{c}\text { (40)-4 (sig) } \\
\text { CGP }\end{array}$ & $\begin{array}{c}\text { (40)-4 (sig) } \\
\text { LM }\end{array}$ & $\begin{array}{c}\text { (40)-4 (sig) } \\
\text { GDX }\end{array}$ \\
\hline Number if Inputs & 40 & 40 & 40 & 40 & 40 & 40 \\
\hline Layer 1 Neurons & 4 & 4 & 4 & 4 & 4 & 4 \\
\hline $\begin{array}{l}\text { Layer } 1 \text { Transfer } \\
\text { Function }\end{array}$ & $L I N$ & LIN & LIN & $S I G$ & $S I G$ & $S I G$ \\
\hline Training Function & $C G P$ & $L M$ & $G D X$ & $C G P$ & $L M$ & $G D X$ \\
\hline Initial Learning Rate & & & 0.02 & & & 0.02 \\
\hline Number of Networks & 3 & 3 & 3 & 3 & 3 & 3 \\
\hline Training Batch Size & $\begin{array}{c}6,000 \\
\text { Symbols } \\
\end{array}$ & $\begin{array}{c}6,000 \\
\text { Symbols }\end{array}$ & $\begin{array}{c}6,000 \\
\text { Symbols }\end{array}$ & $\begin{array}{c}6,000 \\
\text { Symbols }\end{array}$ & $\begin{array}{c}6,000 \\
\text { Symbols } \\
\end{array}$ & $\begin{array}{c}6,000 \\
\text { Symbols }\end{array}$ \\
\hline Training SNR(s) & $\begin{array}{c}-11,-7,-3 \\
\mathrm{~dB}\end{array}$ & $\begin{array}{c}-11,-7,-3 \\
\mathrm{~dB}\end{array}$ & $\begin{array}{c}-11,-7,-3 \\
\mathrm{~dB}\end{array}$ & $\begin{array}{c}-11,-7,-3 \\
\mathrm{~dB}\end{array}$ & $\begin{array}{c}-11,-7,-3 \\
\mathrm{~dB}\end{array}$ & $\begin{array}{c}-11,-7,-3 \\
\mathrm{~dB}\end{array}$ \\
\hline $\begin{array}{l}\text { Training Epochs per } \\
\text { Training Session }\end{array}$ & 150 & 20 & 150 & 150 & 20 & 150 \\
\hline $\begin{array}{l}\text { \# of Training } \\
\text { Sessions }\end{array}$ & 2 & 2 & 2 & 2 & 2 & 2 \\
\hline
\end{tabular}

Table 4-35 Training and test parameters for Figure 4-45

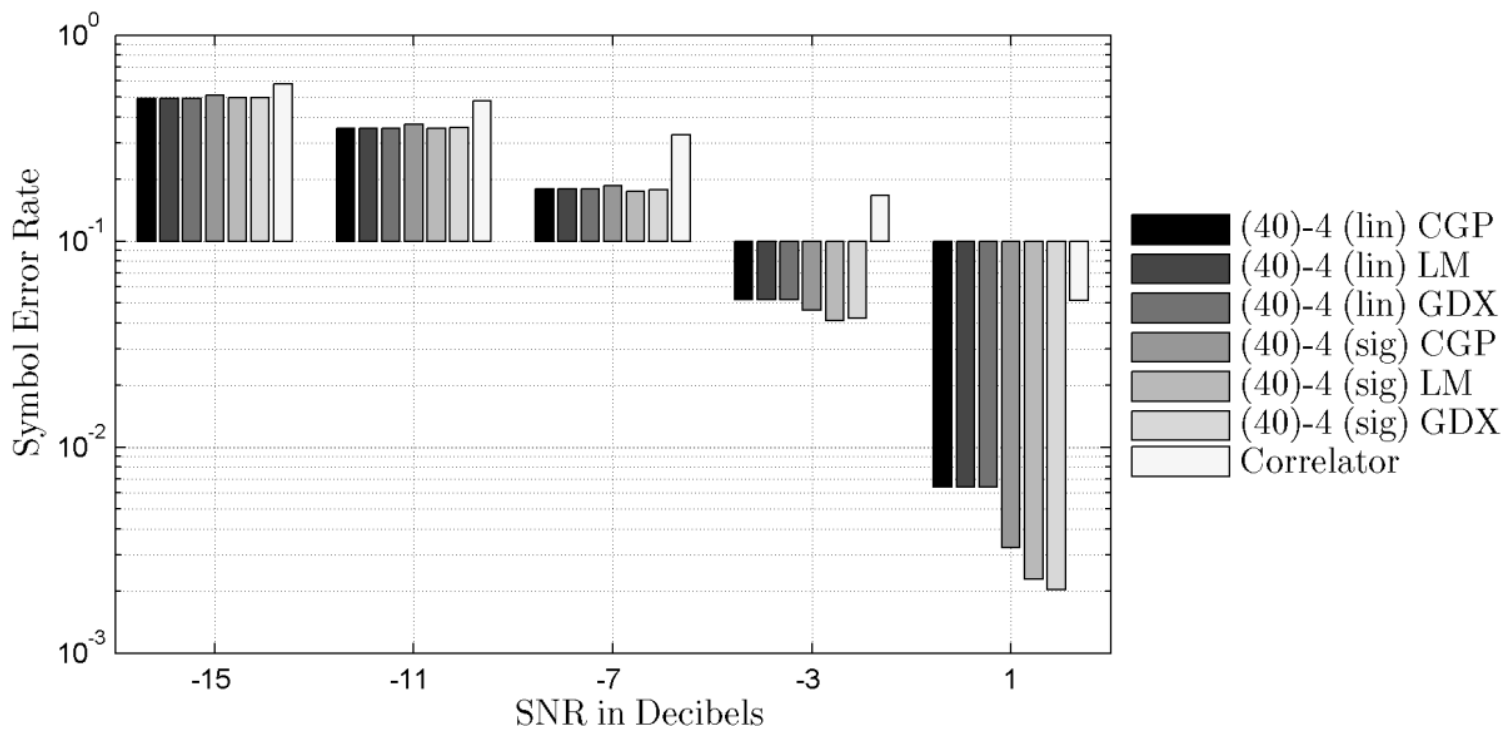

Figure 4-45 Varying Training Algorithm for Single Layer Linear and Non-Linear Networks with Pink Noise 
SNR

\begin{tabular}{|c|c|c|c|c|c|}
\hline Receiver & $-15 \mathrm{~dB}$ & $-11 d B$ & $-7 \mathrm{~dB}$ & $-3 \mathrm{~dB}$ & $1 \mathrm{~dB}$ \\
\hline (40)-4 (lin) CGP & 0.49418 & 0.35273 & 0.17942 & 0.05206 & 0.006383 \\
\hline (40)-4 (lin) LM & 0.49415 & 0.35278 & 0.17944 & 0.05207 & 0.006383 \\
\hline (40)-4 (lin) GDX & 0.49417 & 0.35280 & 0.17945 & 0.05201 & 0.006372 \\
\hline (40)-4 (sig) CGP & 0.51142 & 0.36812 & 0.18618 & 0.04616 & 0.003239 \\
\hline (40)-4 (sig) LM & 0.49489 & 0.35285 & 0.17470 & 0.04117 & 0.002283 \\
\hline (40)-4 (sig) GDX & 0.49696 & 0.35542 & 0.17789 & 0.04215 & 0.002033 \\
\hline Correlator & 0.58063 & 0.47778 & 0.32846 & 0.16653 & 0.051717 \\
\hline \# Symbols Tested & 28,400 & 31,600 & 42,800 & 60,000 & 60,000 \\
\hline
\end{tabular}

Table 4-36 SER Data for Figure 4-45

\subsubsection{Varying Noise Color}

The performances of a few selected networks were evaluated with noise sources

having $\beta$ equal to $0.5,1.0$, and 1.5 . Figure $4-47$ shows the results of setting $\beta$ equal to 0.5 . See Figure 4-38 for the PSD of this noise.

a)

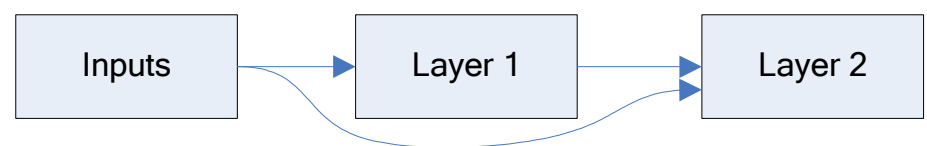

b)

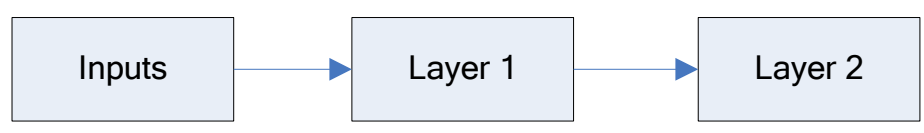

c)

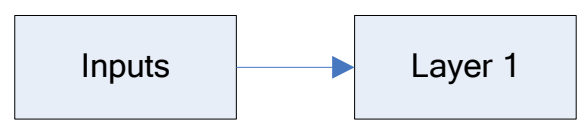

Figure 4-46 Layer connections for neural networks of Figure 4-47, Figure 4-48, and Figure 4-49. a) Connections for 2 layer cascade networks. b) Connections for 2 layer feed forward networks. c)

Connections for single layer networks. 


\begin{tabular}{|c|c|c|c|c|}
\hline Parameter & Value & Value & Value & Value \\
\hline Network Identifier & $(40)-16-4(\mathrm{sig})$ casc & $(40)-16-4(\mathrm{sig}$, lin $)$ & $(40)-4(\mathrm{sig})$ & $(40)-4$ (lin) \\
\hline Number if Inputs & 40 & 40 & 40 & 40 \\
\hline Layer 1 Neurons & 16 & 16 & 4 & 4 \\
\hline Layer 1 Transfer Function & $S I G$ & $S I G$ & $S I G$ & $L I N$ \\
\hline Layer 2 Neurons & 4 & 4 & - & - \\
\hline Layer 2 Transfer Function & $L I N$ & $L I N$ & - & - \\
\hline Training Function & $G D X$ & $G D X$ & $G D X$ & $G D X$ \\
\hline Initial Learning Rate & 0.1 & 0.1 & 0.1 & 0.1 \\
\hline Number of Networks & 3 & 3 & 3 & 3 \\
\hline Training Batch Size & $6,000 \mathrm{Symbols}$ & $6,000 \mathrm{Symbols}$ & $6,000 \mathrm{Symbols}$ & $6,000 \mathrm{Symbols}$ \\
\hline Training SNR(s) & $-11,-7,-3 \mathrm{~dB}$ & $-11,-7,-3 \mathrm{~dB}$ & $-11,-7,-3 \mathrm{~dB}$ & $-11,-7,-3 \mathrm{~dB}$ \\
\hline Training Epochs per & 150 & 150 & 150 & 150 \\
\hline Training Session & 2 & 2 & 2 & 2 \\
\hline \# of Training Sessions & & & & \\
\hline
\end{tabular}

Table 4-37 Training and test parameters for Figure 4-47, Figure 4-48, and Figure 4-49

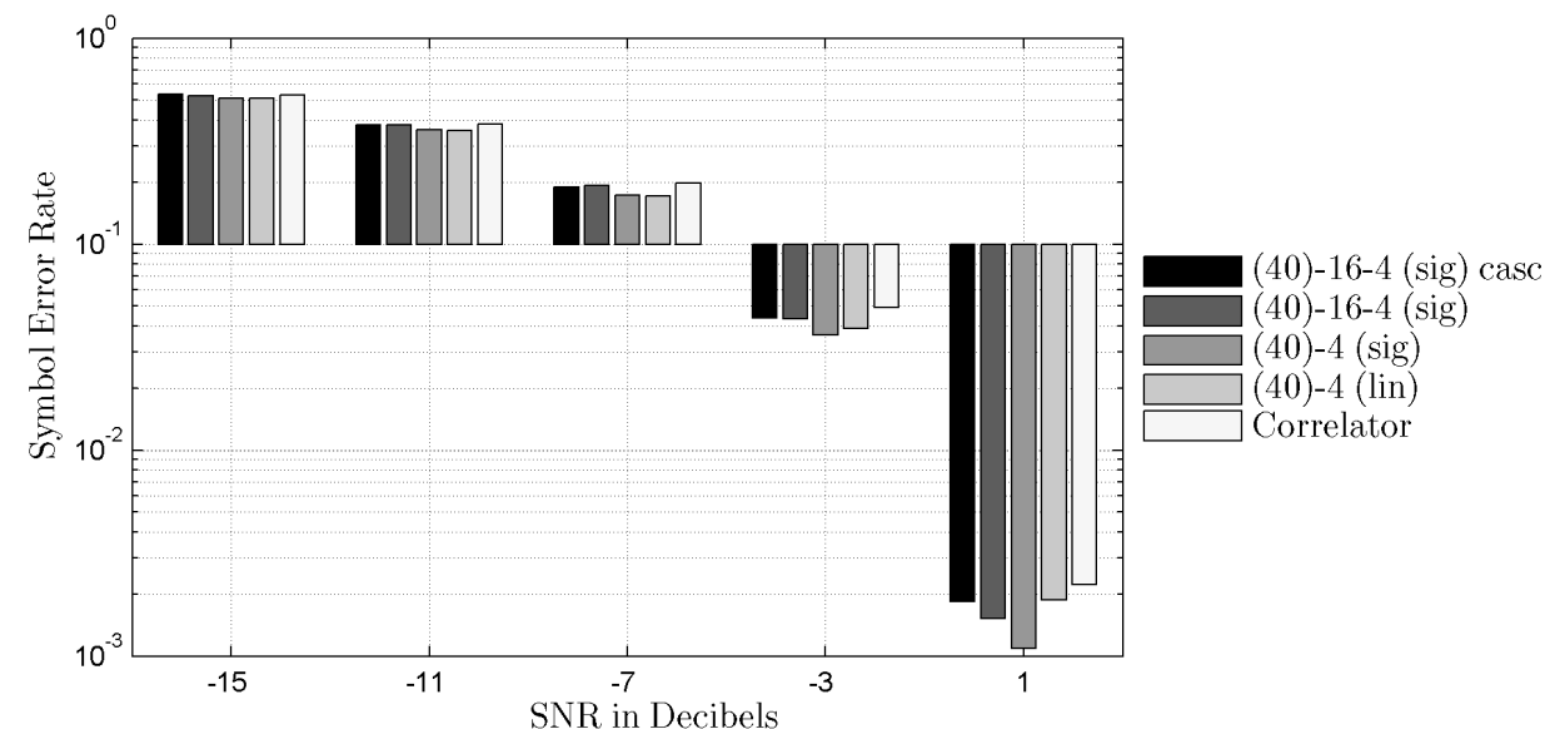

Figure 4-47 Networks Tested With Shaped Noise $($ Beta $=0.5)$ and 4 Symbols 


\section{SNR}

\begin{tabular}{|crrrrr}
\hline Receiver & -15dB & \multicolumn{1}{c}{-11dB } & \multicolumn{1}{c}{-7dB } & -3dB & \multicolumn{1}{c}{ 1dB } \\
\hline (40)-16-4 (sig) casc & 0.53461 & 0.37726 & 0.18857 & 0.04375 & 0.001843 \\
$(40)-16-4(\mathrm{sig})$ & 0.52605 & 0.37952 & 0.19222 & 0.04341 & 0.00152 \\
$(40)-4(\mathrm{sig})$ & 0.51169 & 0.35741 & 0.17219 & 0.03623 & 0.001087 \\
$(40)-4($ lin) & 0.50886 & 0.35600 & 0.17174 & 0.03889 & 0.00187 \\
Correlator & 0.52714 & 0.38319 & 0.19820 & 0.04919 & 0.00222 \\
\hline \# Symbols Tested & 36,000 & 43,000 & 67,400 & 100,000 & 100,000 \\
\hline
\end{tabular}

Table 4-38 SER Data for Figure 4-47

Note that the single layer network having a hyperbolic tangent sigmoid transfer function performed the best. The single layer networks with linear transfer functions performed slightly better than the correlation receiver in this case.

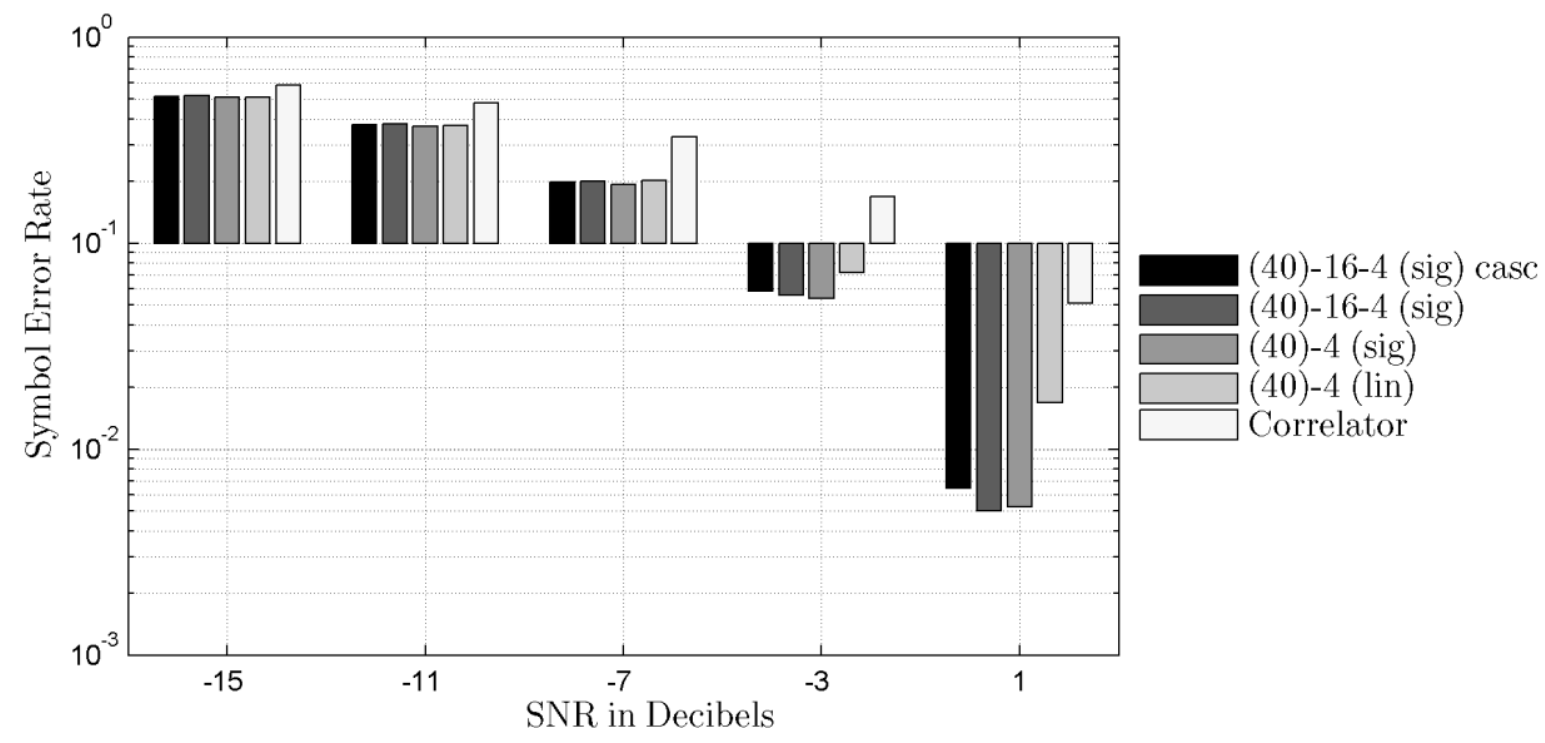

Figure 4-48 Networks Tested With Shaped Noise $($ Beta $=1.0)$ and 4 Symbols 


\section{SNR}

\begin{tabular}{|crrrrr}
\hline Receiver & -15dB & \multicolumn{1}{c}{-11dB } & \multicolumn{1}{c}{-7dB } & -3dB & \multicolumn{1}{c}{ 1dB } \\
\hline (40)-16-4 (sig) casc & 0.51707 & 0.37509 & 0.19692 & 0.05844 & 0.00644 \\
(40)-16-4 (sig) & 0.51807 & 0.37860 & 0.19957 & 0.05594 & 0.005027 \\
$(40)-4(\mathrm{sig})$ & 0.50879 & 0.36975 & 0.19242 & 0.05388 & 0.00526 \\
$(40)-4($ lin) & 0.50891 & 0.37062 & 0.20066 & 0.07192 & 0.016857 \\
Correlator & 0.58258 & 0.47726 & 0.32848 & 0.16786 & 0.05092 \\
\hline \# Symbols Tested & 36,000 & 42,400 & 62,400 & 100,000 & 100,000 \\
\hline
\end{tabular}

Table 4-39 SER Data for Figure 4-48

Figure 4-48 shows the results of increasing $\beta$ to 1.0. Figure 4-39 shows the PSD of the noise source. At this point, we can see that the non-linear networks outperform both the correlation receiver and the single layer linear network.

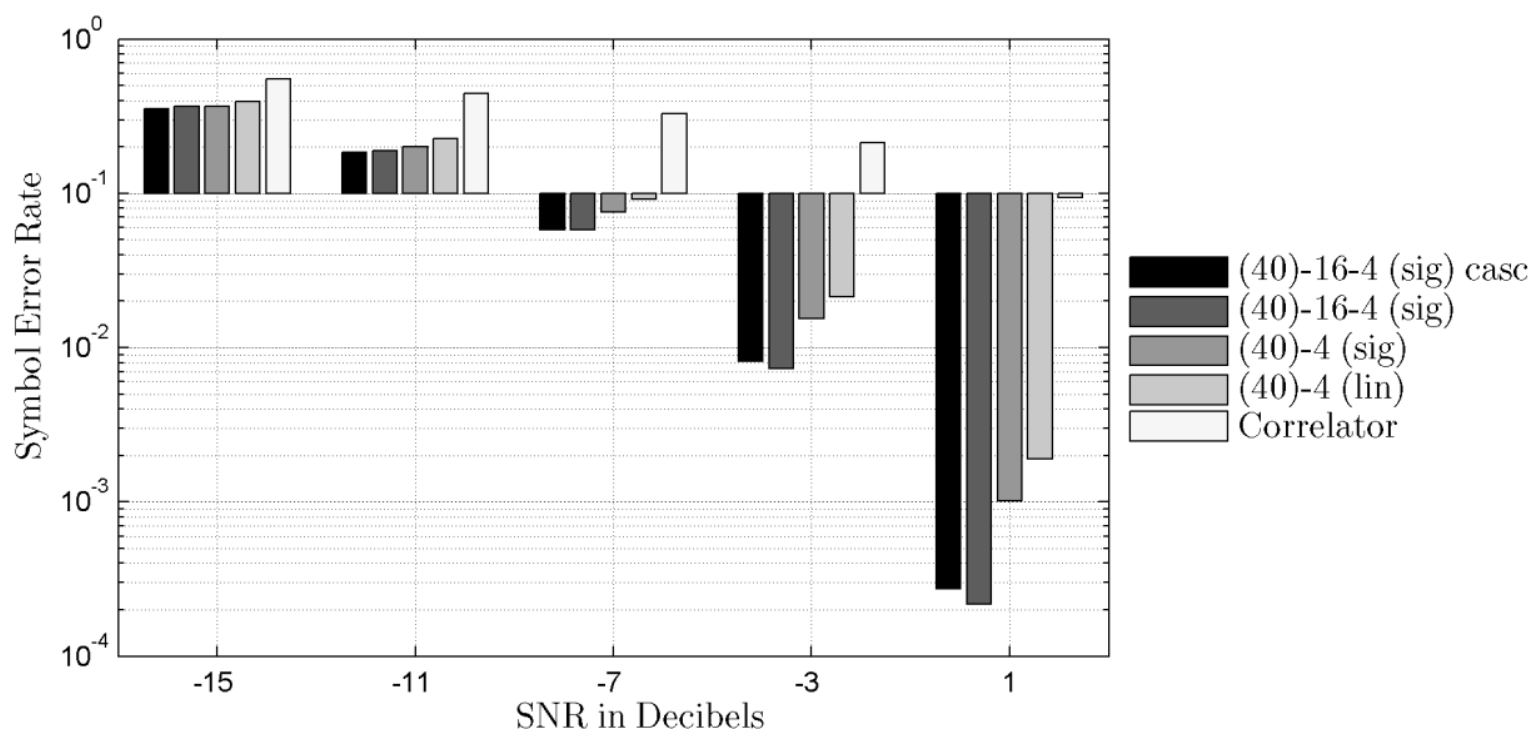

Figure 4-49 Networks Tested With Shaped Noise $($ Beta $=1.5)$ and 4 Symbols 


\begin{tabular}{|cccccc}
\hline & \multicolumn{6}{c}{ SNR } \\
\hline Receiver & $\mathbf{- 1 5 d B}$ & $\mathbf{- 1 1 d B}$ & $\mathbf{- 7 d B}$ & $\mathbf{- 3 d B}$ & 1dB \\
\hline (40)-16-4 (sig) casc & 0.35446 & 0.18323 & 0.05827 & 0.00817 & 0.000273 \\
$(40)-16-4(\mathrm{sig})$ & 0.36554 & 0.18814 & 0.05828 & 0.00730 & 0.000217 \\
$(40)-4(\mathrm{sig})$ & 0.36495 & 0.19970 & 0.07539 & 0.01541 & 0.001013 \\
$(40)-4(\mathrm{lin})$ & 0.39276 & 0.22687 & 0.09139 & 0.02135 & 0.001897 \\
Correlator & 0.55132 & 0.44261 & 0.32662 & 0.21204 & 0.09411 \\
\hline \# Symbols Tested & 43,200 & 65,400 & 100,000 & 100,000 & 100,000 \\
\hline
\end{tabular}

Table 4-40 SER Data for Figure 4-49

The receivers of Figure 4-49 were simulated using $\beta$ equal to 1.5. The 2 layer nonlinear networks have considerably better performance than both of the single layer networks and the correlation receiver. The PSD of the corrupting noise source for this simulation is shown in Figure 4-40.

\subsubsection{Frequency Domain Generated Shaped Noise}

The next three figures show the results of testing with the noise sources shown in Figure 4-41, Figure 4-42, and Figure 4-43. The difference in performance of the ANN receivers versus the correlation receiver increases in each subsequent test.

a)

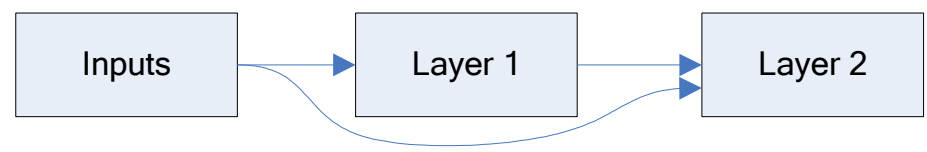

b)

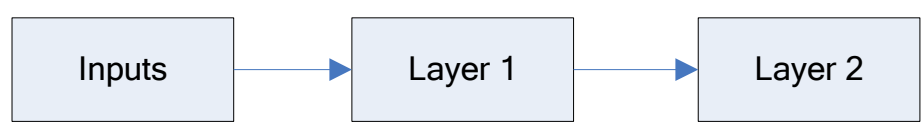

c)

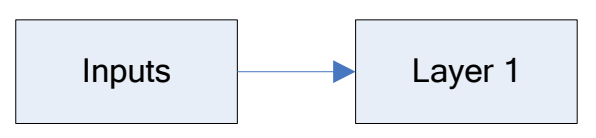

Figure 4-50 Layer connections for neural networks of Figure 4-51, Figure 4-52, and Figure 4-53. a) Connections for 2 layer cascade networks. b) Connections for 2 layer feed forward networks. c) Connections for single layer networks. 


\begin{tabular}{|c|c|c|c|c|}
\hline Parameter & Value & Value & Value & Value \\
\hline Network Identifier & $(40)-16-4($ sig) casc & $(40)-16-4($ sig,lin) & $(40)-4($ sig) & $(40)-4$ (lin) \\
\hline Number if Inputs & 40 & 40 & 40 & 40 \\
\hline Layer 1 Neurons & 16 & 16 & 4 & 4 \\
\hline Layer 1 Transfer Function & SIG & SIG & $S I G$ & LIN \\
\hline Layer 2 Neurons & 4 & 4 & - & - \\
\hline Layer 2 Transfer Function & LIN & $L I N$ & - & - \\
\hline Training Function & $G D X$ & $G D X$ & $G D X$ & $G D X$ \\
\hline Initial Learning Rate & 0.1 & 0.1 & 0.1 & 0.1 \\
\hline Number of Networks & 3 & 3 & 3 & 3 \\
\hline Training Batch Size & 6,000 Symbols & 6,000 Symbols & 6,000 Symbols & 6,000 Symbols \\
\hline Training SNR(s) & $-11,-7,-3 \mathrm{~dB}$ & $-11,-7,-3 \mathrm{~dB}$ & $-11,-7,-3 \mathrm{~dB}$ & $-11,-7,-3 \mathrm{~dB}$ \\
\hline Training Epochs per & 150 & 150 & 150 & 150 \\
\hline Training Session & 2 & 2 & 2 & 2 \\
\hline \# of Training Sessions & & &
\end{tabular}

Table 4-41 Training and test parameters for Figure 4-51, Figure 4-52, and Figure 4-53

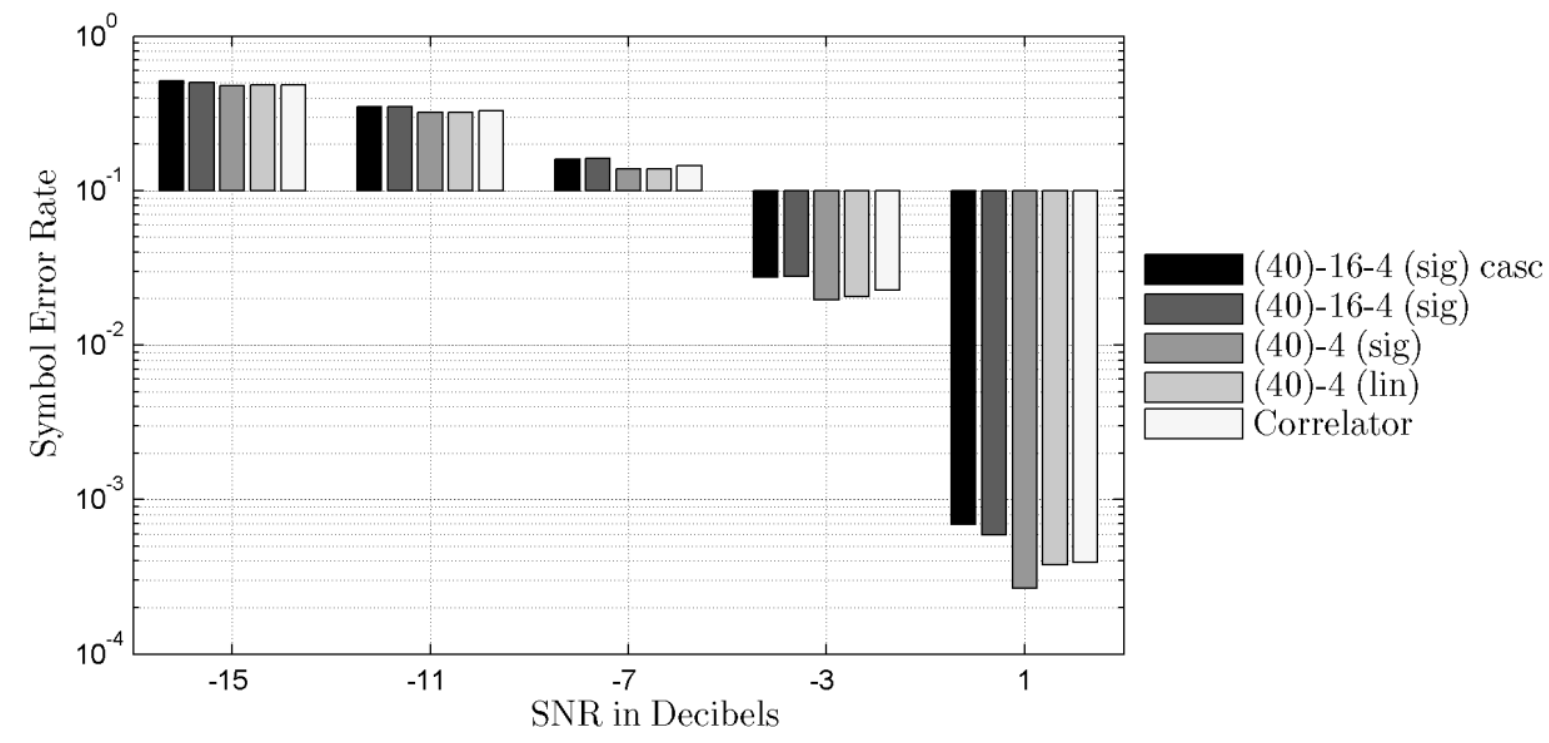

Figure 4-51 Networks Tested with Shaped Noise of Figure 4-41 $($ Beta $=0.5$, Center $=10 \mathrm{~Hz}$, Width $=4 \mathrm{~Hz})$ 


\section{SNR}

\begin{tabular}{|crrrrr}
\hline Receiver & -15dB & \multicolumn{1}{c}{-11dB } & \multicolumn{1}{c}{-7dB } & -3dB & \multicolumn{1}{c}{ 1dB } \\
\hline (40)-16-4 (sig) casc & 0.51352 & 0.34831 & 0.15945 & 0.02744 & 0.000693 \\
$(40)-16-4(\mathrm{sig})$ & 0.50029 & 0.34727 & 0.16210 & 0.02778 & 0.00059 \\
$(40)-4(\mathrm{sig})$ & 0.47923 & 0.31898 & 0.13723 & 0.01953 & 0.000267 \\
$(40)-4($ lin) & 0.48022 & 0.31882 & 0.13795 & 0.02068 & 0.00038 \\
Correlator & 0.48532 & 0.32959 & 0.14532 & 0.02270 & 0.00039 \\
\hline \# Symbols Tested & 37,200 & 45,800 & 78,800 & 100,000 & 100,000 \\
\hline
\end{tabular}

Table 4-42 SER Data for Figure 4-51

All the networks of Figure 4-51 performed similarly to the correlation receiver. The single-layer network with the SIG transfer function performed slightly better than all the other receivers.

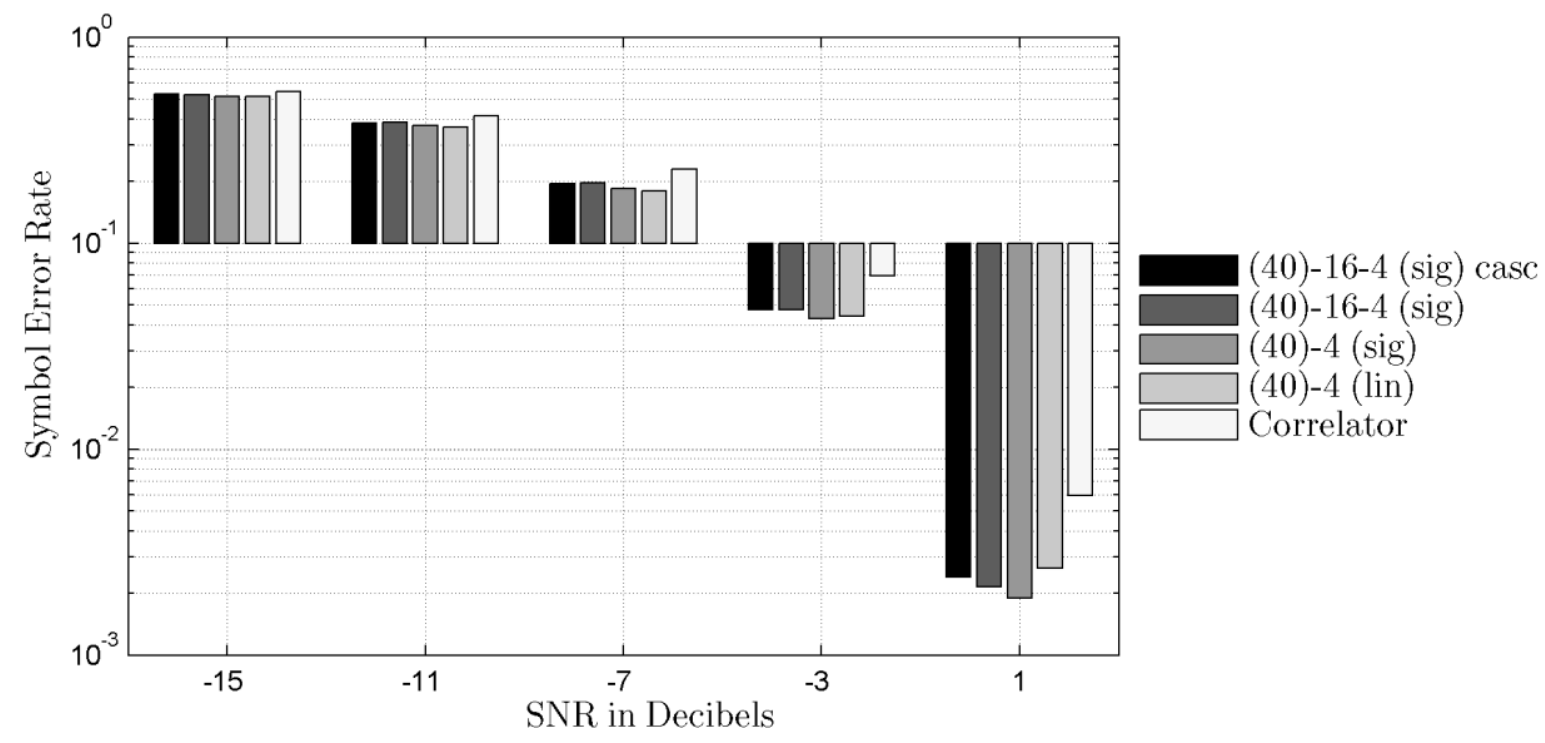

Figure 4-52 Networks Tested with Shaped Noise of Figure 4-42 $($ Beta $=1.0$, Center $=10 \mathrm{~Hz}$, Width $=4 \mathrm{~Hz})$ 
SNR

\begin{tabular}{|ccccrr}
\hline Receiver & -15dB & -11dB & -7dB & -3dB & \multicolumn{1}{c}{ 1dB } \\
\hline (40)-16-4 (sig) casc & 0.53082 & 0.38351 & 0.19372 & 0.04764 & 0.00238 \\
$(40)-16-4(\mathrm{sig})$ & 0.52655 & 0.38734 & 0.19621 & 0.04742 & 0.002143 \\
$(40)-4(\mathrm{sig})$ & 0.51476 & 0.37048 & 0.18314 & 0.04285 & 0.001883 \\
$(40)-4(\mathrm{lin})$ & 0.51293 & 0.36599 & 0.17937 & 0.04398 & 0.00264 \\
Correlator & 0.54171 & 0.41370 & 0.22940 & 0.06939 & 0.00595 \\
\hline \# Symbols Tested & 36,200 & 42,400 & 64,600 & 100,000 & 100,000 \\
\hline
\end{tabular}

Table 4-43 SER Data for Figure 4-52

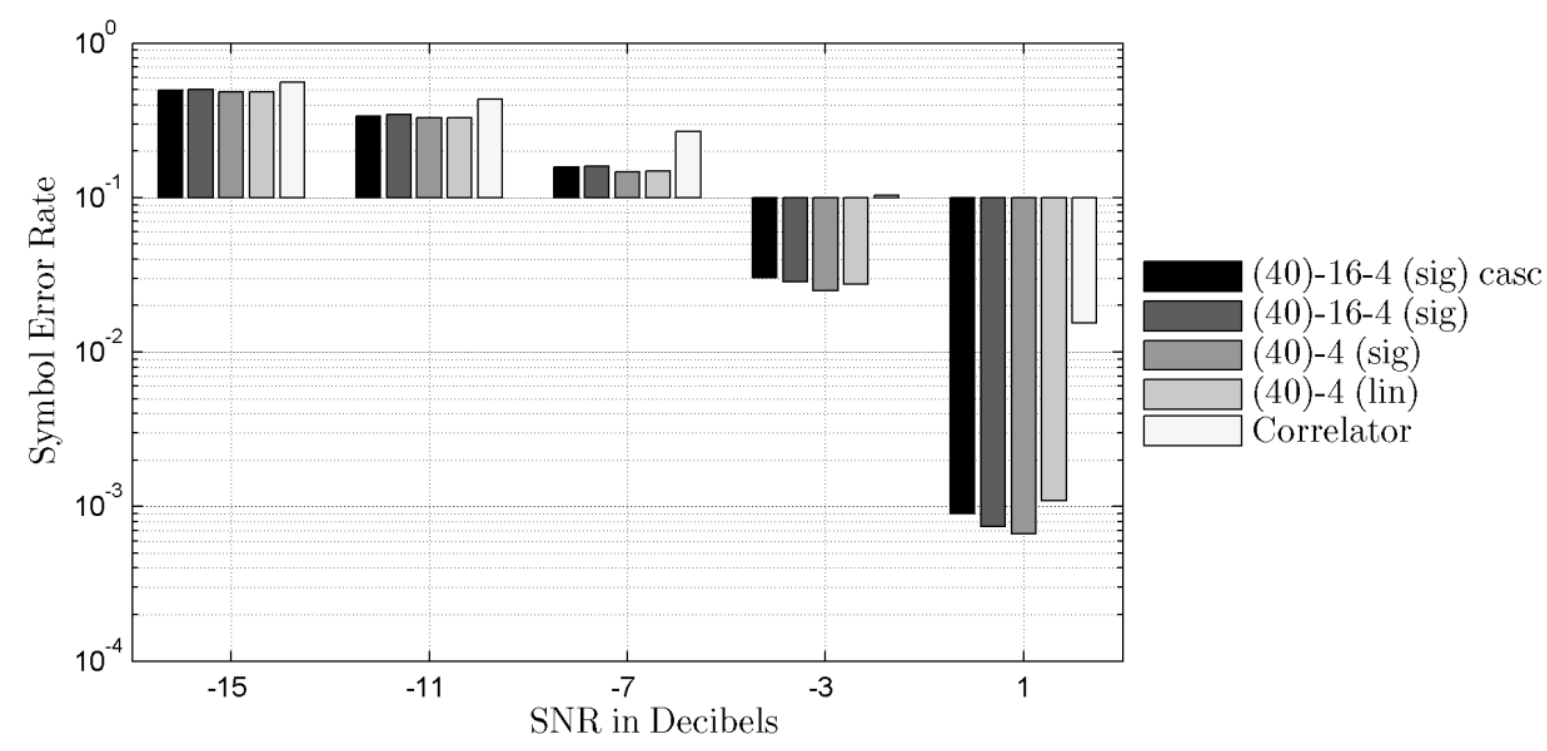

Figure 4-53 Networks Tested with Shaped Noise of Figure 4-43 $($ Beta $=1.5$, Center $=10 \mathrm{~Hz}$, Width $=4 \mathrm{~Hz})$

\begin{tabular}{|ccccrr}
\hline \multicolumn{7}{c}{ SNR } \\
\hline Receiver & $\mathbf{- 1 5 d B}$ & $\mathbf{- 1 1 d B}$ & $\mathbf{- 7 d B}$ & -3dB & \multicolumn{1}{c}{ 1dB } \\
\hline (40)-16-4 (sig) casc & 0.49594 & 0.33827 & 0.15680 & 0.03043 & 0.000903 \\
$(40)-16-4(\mathrm{sig})$ & 0.49865 & 0.34467 & 0.16028 & 0.02866 & 0.000747 \\
$(40)-4(\mathrm{sig})$ & 0.48542 & 0.32908 & 0.14689 & 0.02484 & 0.00067 \\
$(40)-4($ lin) & 0.48228 & 0.32865 & 0.14830 & 0.02760 & 0.001093 \\
Correlator & 0.55841 & 0.43395 & 0.26731 & 0.10288 & 0.01542 \\
\hline \# Symbols Tested & 37,000 & 44,800 & 74,600 & 100,000 & 100,000 \\
\hline
\end{tabular}

Table 4-44 SER Data for Figure 4-53 
It is interesting to note that the performance of the neural networks increased as the Beta value used in the synthesized shaped noise increased from 1.0 to 1.5 . Meanwhile, the performance of the correlation receiver decreased.

\subsection{Simulations Using Non-AWGN and 8 Symbols}

The simulations resulting in Figure 4-47, Figure 4-48, and Figure 4-49 were repeated with a symbol set containing 8 symbols.

a)

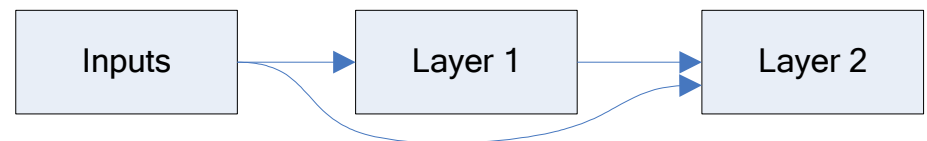

b)

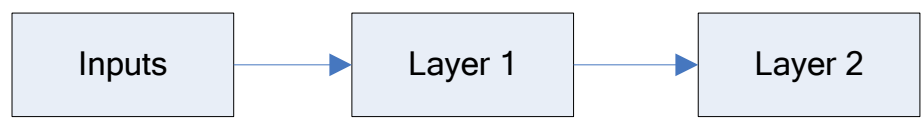

c)

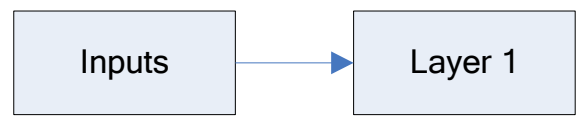

Figure 4-54 Layer connections for neural networks of Figure 4-55, Figure 4-56, and Figure 4-57. a) Connections for 2 layer cascade networks. b) Connections for 2 layer feed forward networks. c) Connections for single layer networks. 


\begin{tabular}{|c|c|c|c|c|}
\hline Parameter & Value & Value & Value & Value \\
\hline Network Identifier & (80)-16-8(sig) casc & (80)-16-8 (sig,lin) & (80)-8 (sig) & (80)-8 (lin) \\
\hline Number if Inputs & 80 & 80 & 80 & 80 \\
\hline Layer 1 Neurons & 16 & 16 & 8 & 8 \\
\hline Layer 1 Transfer Function & $S I G$ & $S I G$ & $S I G$ & LIN \\
\hline Layer 2 Neurons & 8 & 8 & - & - \\
\hline Layer 2 Transfer Function & LIN & LIN & - & - \\
\hline Training Function & $G D X$ & $G D X$ & $G D X$ & $G D X$ \\
\hline Initial Learning Rate & 0.1 & 0.1 & 0.1 & 0.1 \\
\hline Number of Networks & 3 & 3 & 3 & 3 \\
\hline Training Batch Size & 12,000 Symbols & 12,000 Symbols & 12,000 Symbols & 12,000 Symbols \\
\hline Training SNR(s) & $-11,-7,-3 \mathrm{~dB}$ & $-11,-7,-3 \mathrm{~dB}$ & $-11,-7,-3 \mathrm{~dB}$ & $-11,-7,-3 \mathrm{~dB}$ \\
\hline $\begin{array}{c}\text { Training Epochs per Training } \\
\text { Session }\end{array}$ & 150 & 150 & 150 & 150 \\
\hline \# of Training Sessions & 2 & 2 & 2 & 2 \\
\hline
\end{tabular}

Table 4-45 Training and test parameters for Figure 4-55, Figure 4-56, and Figure 4-57

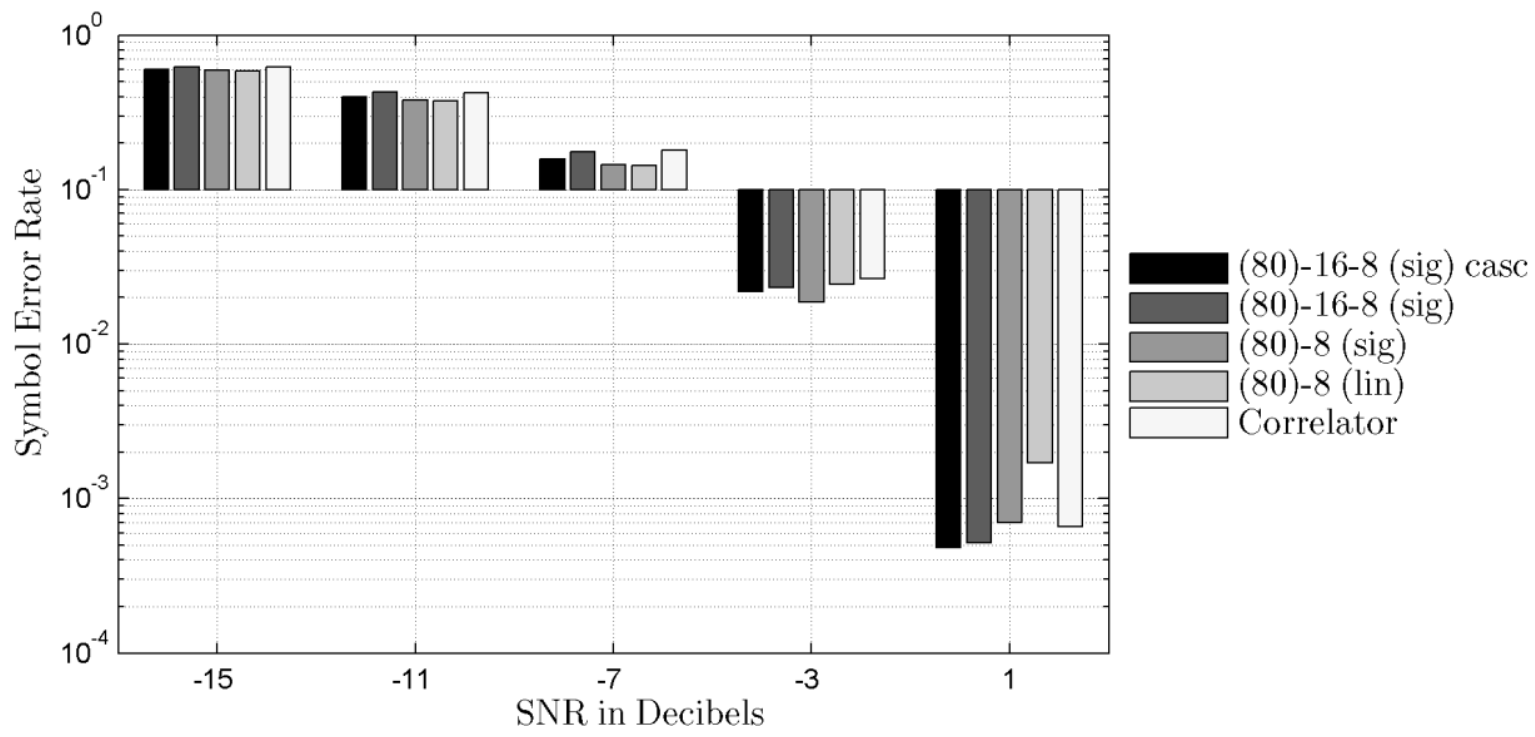

Figure 4-55 Networks Tested With Shaped Noise $($ Beta $=0.5)$ and 8 Symbols 


\section{SNR}

\begin{tabular}{|crrrrr|}
\hline Receiver & -15dB & \multicolumn{1}{c|}{-11dB } & \multicolumn{1}{c|}{-7dB } & -3dB & \multicolumn{1}{c|}{ 1dB } \\
\hline (80)-16-8 (sig) casc & 0.60152 & 0.39587 & 0.15788 & 0.02181 & 0.000483 \\
$(80)-16-8(\mathrm{sig})$ & 0.61962 & 0.42601 & 0.17472 & 0.02316 & 0.00052 \\
$(80)-8(\mathrm{sig})$ & 0.58953 & 0.38101 & 0.14486 & 0.01864 & 0.000697 \\
$(80)-8($ lin) & 0.58369 & 0.37665 & 0.14255 & 0.02450 & 0.00171 \\
Correlator & 0.62227 & 0.42318 & 0.17885 & 0.02649 & 0.00066 \\
\hline \# Symbols Tested & 34,400 & 42,400 & 78,400 & 100,000 & 100,000 \\
\hline
\end{tabular}

Table 4-46 SER Data for Figure 4-55

All receivers in Figure 4-55 performed similarly. The single layer linear network performed slightly worse than the others when the SNR was set to $8 \mathrm{~dB}$. The best performing network was a single layer network using SIG transfer functions.

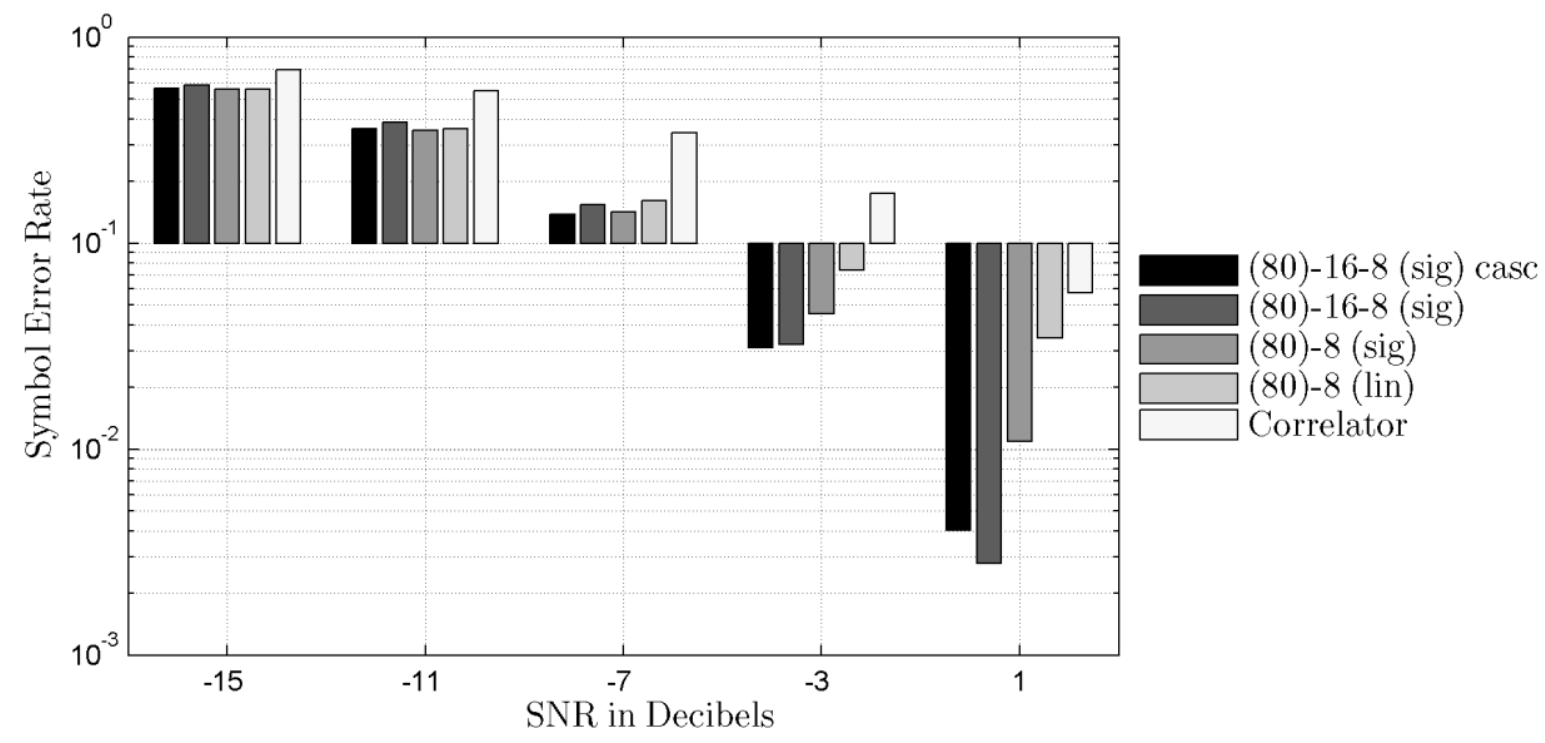

Figure 4-56 Networks Tested With Shaped Noise $($ Beta $=1.0)$ and 8 Symbols 


\section{SNR}

\begin{tabular}{|crrrrr|}
\hline Receiver & -15dB & \multicolumn{1}{c|}{-11dB } & \multicolumn{1}{c|}{-7dB } & -3dB & \multicolumn{1}{c|}{ 1dB } \\
\hline (80)-16-8 (sig) casc & 0.56262 & 0.35798 & 0.13812 & 0.03115 & 0.004047 \\
$(80)-16-8(\mathrm{sig})$ & 0.58650 & 0.38543 & 0.15399 & 0.03233 & 0.002787 \\
$(80)-8(\mathrm{sig})$ & 0.55898 & 0.35296 & 0.14166 & 0.04555 & 0.010883 \\
$(80)-8($ lin) & 0.56024 & 0.35859 & 0.16072 & 0.07399 & 0.0346 \\
Correlator & 0.69095 & 0.54705 & 0.34433 & 0.17362 & 0.05741 \\
\hline \# Symbols Tested & 34,800 & 44,400 & 79,600 & 100,000 & 100,000 \\
\hline
\end{tabular}

Table 4-47 SER Data for Figure 4-56

The simulation results of Figure 4-56 show the increased performance of the 2-layer neural network receivers over the correlation receiver, and single layer networks.

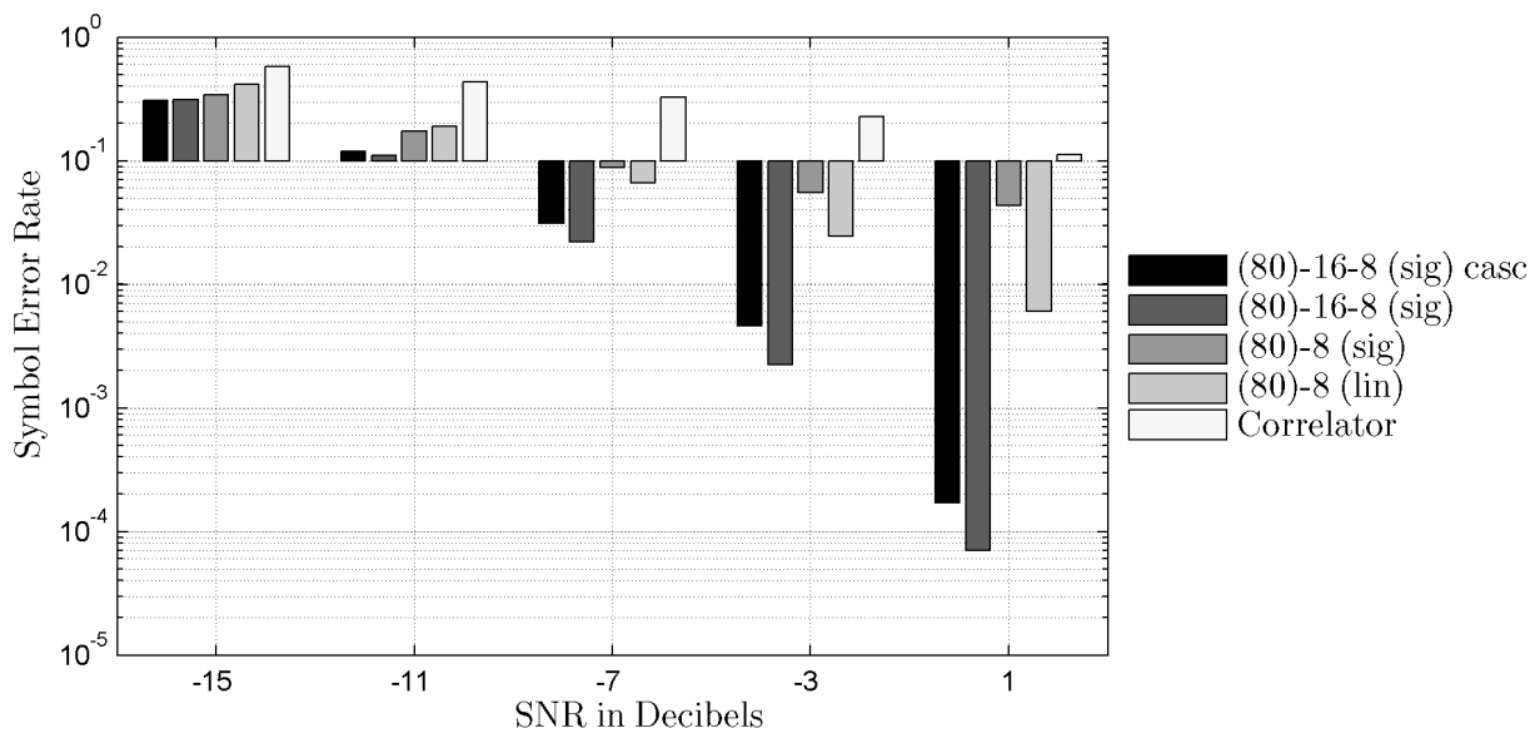

Figure 4-57 Networks Tested With Shaped Noise $($ Beta $=1.5)$ and 8 Symbols 


\section{SNR}

\begin{tabular}{|c|c|c|c|c|c|}
\hline Receiver & $-15 \mathrm{~dB}$ & $-11 d B$ & $-7 \mathrm{~dB}$ & $-3 \mathrm{~dB}$ & $1 \mathrm{~dB}$ \\
\hline (80)-16-8 (sig) casc & 0.30792 & 0.11872 & 0.03130 & 0.00459 & 0.00017 \\
\hline (80)-16-8 (sig) & 0.31329 & 0.11064 & 0.02205 & 0.00224 & 0.00007 \\
\hline (80)-8 (sig) & 0.34063 & 0.17201 & 0.08774 & 0.05509 & 0.043663 \\
\hline (80)-8 (lin) & 0.41352 & 0.19016 & 0.06616 & 0.02462 & 0.00608 \\
\hline Correlator & 0.57385 & 0.43522 & 0.32744 & 0.22746 & 0.11129 \\
\hline \# Symbols Tested & 48,000 & 98,000 & 100,000 & 100,000 & 100,000 \\
\hline
\end{tabular}

Table 4-48 SER Data for Figure 4-57

Figure 4-57 shows the increasing gap in performance between the 2-layer neural network receivers and the other receivers as the noise further deviates from AWGN. The reduced performance exhibited by the single layer network with the SIG transfer function is due to one of the 3 networks tested not performing well. The other two instances of that network had performances that were second best out of all the network architectures. 


\subsection{Simulations Using Field File \#1}
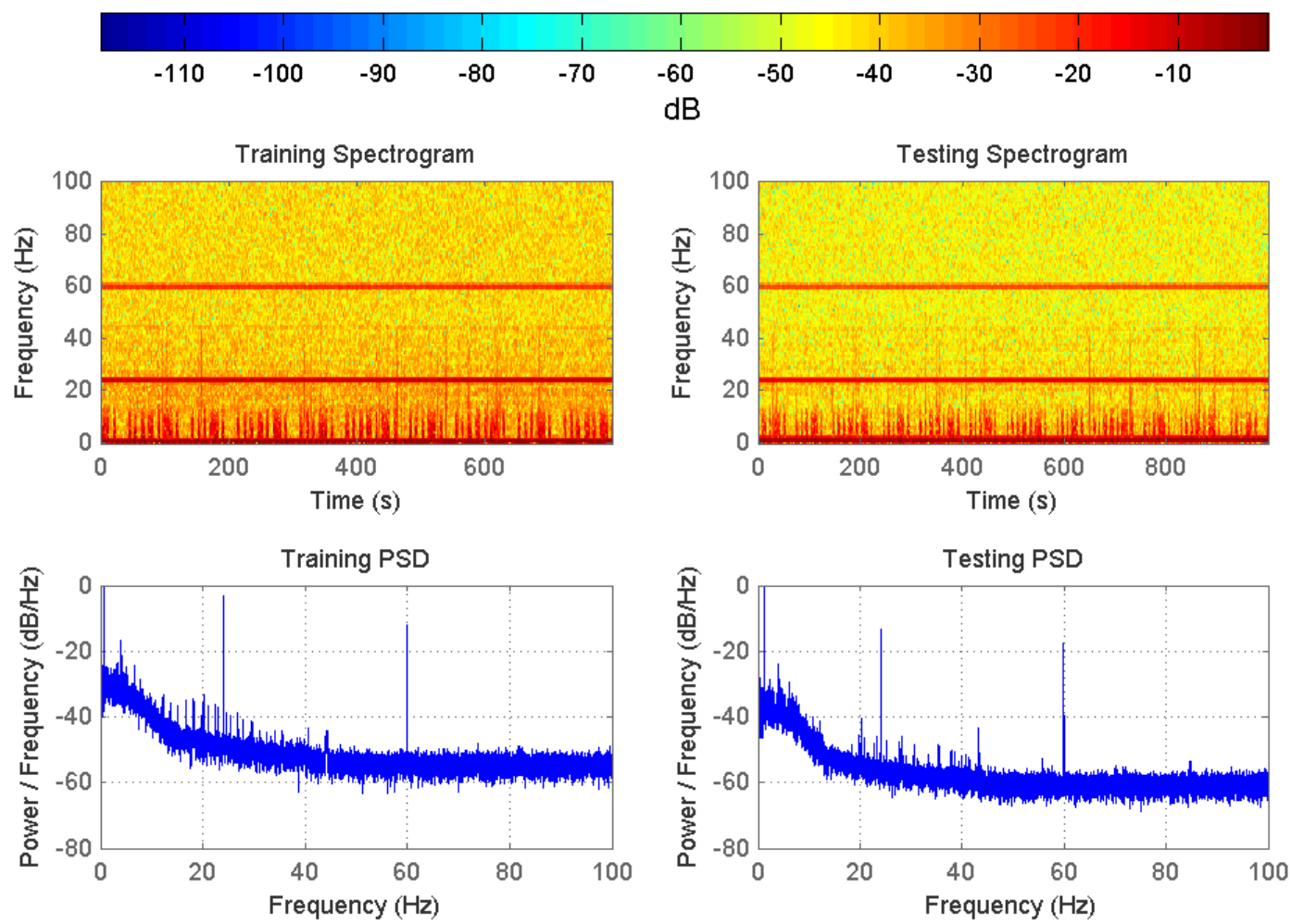

Figure 4-58 Frequency Content of Field File \#1. The power spectral density plots in the $2^{\text {nd }}$ row are generated from the exact same data as their respective spectrograms in the $1^{\text {st }}$ row.

Each of the noise files used in this thesis were obtained from actual drilling sites within the United States by Scientific Drilling International. The data was collected by recording the voltage between a metal stake driven into the ground and the drill pipe. The sampled noise data of Field File \#1 contains large spectral peaks at $60 \mathrm{~Hz}, 22 \mathrm{~Hz}$, and near $1 \mathrm{~Hz}$. There is also considerable energy in the $1 \mathrm{~Hz}$ to $15 \mathrm{~Hz}$ range that is caused by the pulsing of an EM MWD tool. The frequency content of the signal does not vary noticeably over time, or between the samples used for training vs. testing. 


\subsubsection{Training and Testing With The Same Noise Data}

The first set of simulations was performed using the same noise data file as was used to train the networks. This was done to show what should be the best-case scenario for the neural network receivers. The frequency content of the noise data file used for both training and testing is shown on the left-hand side of Figure 4-58. Note that even though the same noise data file was used for training and testing, each noise sample was chosen at random from the noise file during testing. This coupled with the random generation of symbols being transmitted prevents the networks from performing well by purely memorizing the training data. In other words, the exact same inputs and outputs were not used for training and testing; hence, the networks still must be able to generalize to perform well.

a)

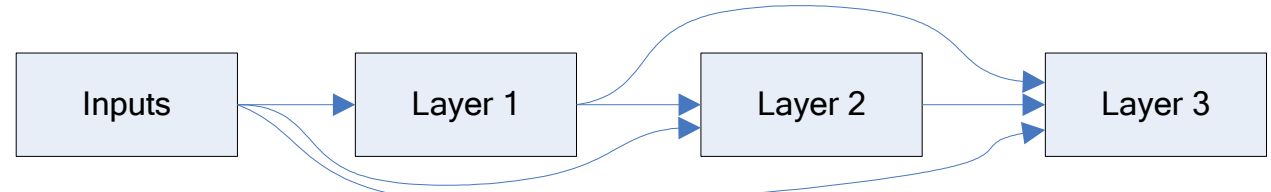

b)

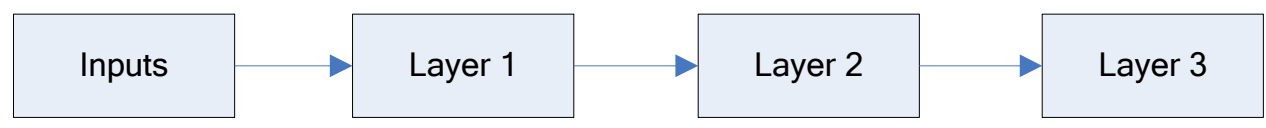

c)

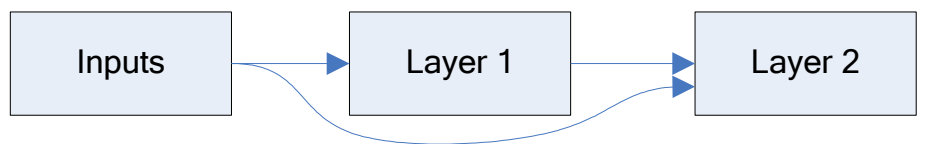

d)

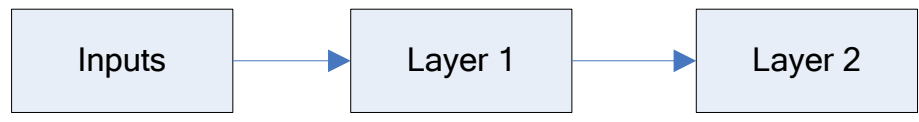

e)

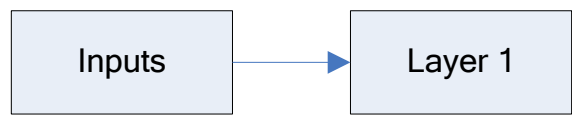

Figure 4-59 Layer connections for neural networks used with field file simulations. a) Connections for 3 layer cascade networks. b) Connections for 3 layer feed forward networks. c) Connections for 2 layer cascade networks. d) Connections for 2 layer feed forward networks. e) Connections for single layer networks. 


\begin{tabular}{|c|c|c|c|c|c|c|}
\hline Parameter & Value & Value & Value & Value & Value & Value \\
\hline Network Identifier & $\begin{array}{c}(40)-8-16-4 \\
\text { casc }\end{array}$ & $\begin{array}{c}(40)-8-16-4 \\
(40)-16-4 \\
\text { casc }\end{array}$ & $\begin{array}{c}(40)-16-4 \\
(40)-4\end{array}$ & $(40)-4$ (lin) \\
\hline Number if Inputs & 40 & 40 & 40 & 40 & 40 & 40 \\
\hline Layer 1 Neurons & 8 & 8 & 16 & 16 & 4 & 4 \\
\hline $\begin{array}{c}\text { Layer 1 Transfer } \\
\text { Function }\end{array}$ & $S I G$ & $S I G$ & $S I G$ & $S I G$ & $S I G$ & LIN \\
\hline Layer 2 Neurons & 16 & 16 & 4 & 4 & - & - \\
\hline $\begin{array}{c}\text { Layer 2 Transfer } \\
\text { Function }\end{array}$ & $S I G$ & $S I G$ & $S I G$ & $S I G$ & - & - \\
\hline Layer 3 Neurons & 4 & 4 & - & - & - & - \\
\hline $\begin{array}{c}\text { Layer 3 Transfer } \\
\text { Function }\end{array}$ & $S I G$ & $S I G$ & - & - & - & - \\
\hline Training Function & $G D X$ & $G D X$ & $G D X$ & $G D X$ & $G D X$ & $G D X$ \\
\hline Initial Learning Rate & 0.1 & 0.1 & 0.1 & 0.1 & 0.1 & 0.1 \\
\hline Number of Networks & 3 & 3 & 3 & 3 & 3 & 3 \\
\hline $\begin{array}{c}\text { Training Batch Size } \\
\text { Symbols }\end{array}$ & 6,000 & 6,000 & 6,000 & 6,000 & 6,000 & 6,000 \\
\hline Training SNR(s) & $-4,0,4 \mathrm{~dB}$ & $-4,0,4 \mathrm{~dB}$ & $-4,0,4 \mathrm{~dB}$ & $-4,0,4 \mathrm{~dB}$ & $-4,0,4 \mathrm{~dB}$ & $-4,0,4 \mathrm{~dB}$ \\
\hline $\begin{array}{c}\text { Training Epochs per } \\
\text { Training Session }\end{array}$ & 150 & 150 & 150 & 150 & 150 & 150 \\
\hline $\begin{array}{c}\text { \# of Training } \\
\text { Sessions }\end{array}$ & 2 & 2 & 2 & 2 & 2 & 2 \\
\hline
\end{tabular}

Table 4-49 Training and test parameters for field file simulations

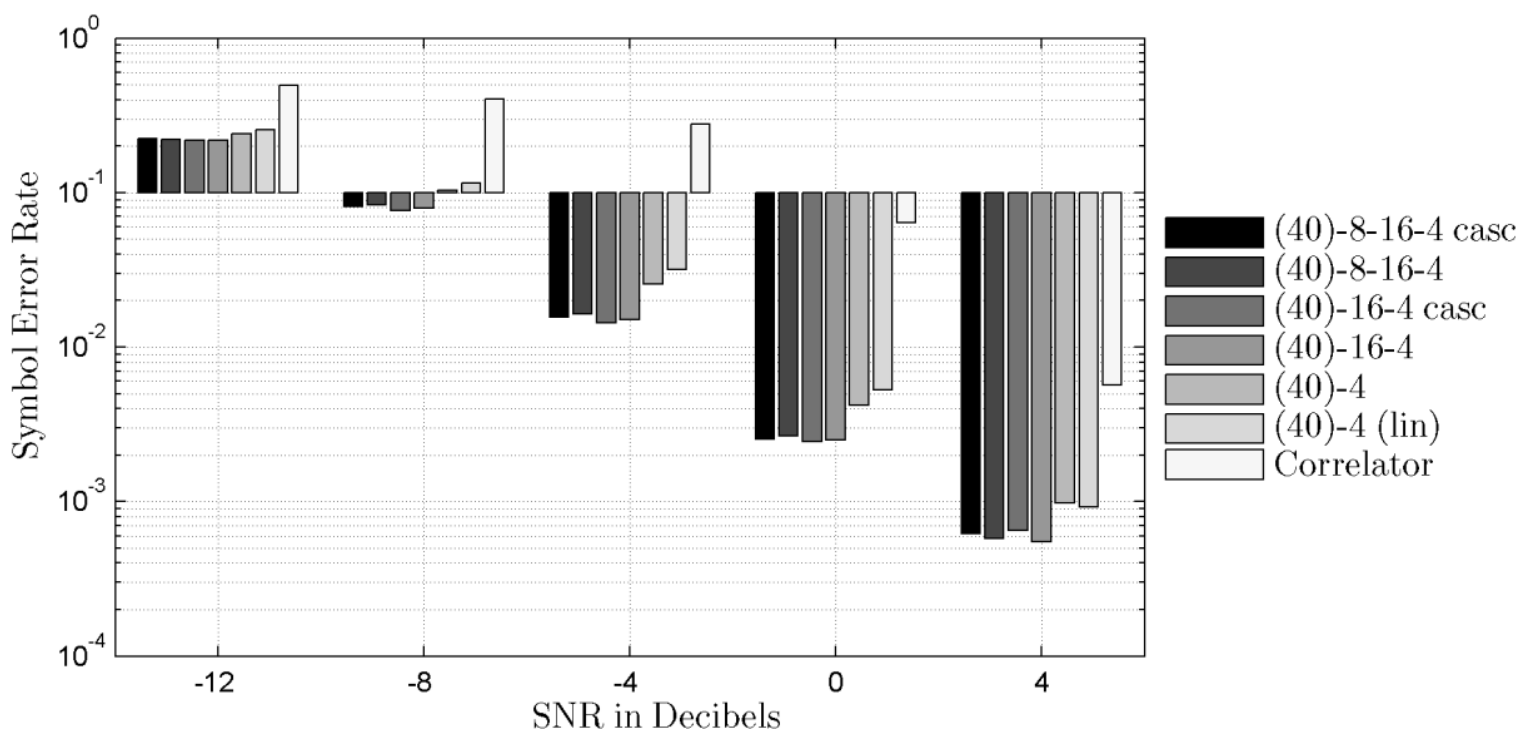

Figure 4-60 Same Training and Test Noise With Field File \#1 and 4 Symbols 


\section{SNR}

\begin{tabular}{|clllll|}
\hline Receiver & $\mathbf{- 1 2}$ & $\mathbf{- 8}$ & $\mathbf{- 4}$ & $\mathbf{0}$ & $\mathbf{4}$ \\
\hline (40)-8-16-4 casc & 0.22282 & 0.08094 & 0.01554 & 0.00255 & 0.00062 \\
$(40)-8-16-4$ & 0.22128 & 0.08357 & 0.01637 & 0.00265 & 0.00057 \\
$(40)-16-4$ casc & 0.21884 & 0.07693 & 0.01436 & 0.00244 & 0.00065 \\
$(40)-16-4$ & 0.21699 & 0.07899 & 0.01505 & 0.00249 & 0.00055 \\
$(40)-4$ & 0.24039 & 0.10276 & 0.02547 & 0.00418 & 0.00098 \\
(40)-4 (lin) & 0.25645 & 0.11455 & 0.03178 & 0.00530 & 0.00092 \\
Correlator & 0.49392 & 0.40533 & 0.27702 & 0.06395 & 0.00568 \\
\hline \# Symbols Tested & 59,400 & 100,000 & 100,000 & 100,000 & 100,000 \\
\hline
\end{tabular}

Table 4-50 SER Data for Figure 4-60

All the neural network receivers performed better than both the correlation receiver and the single layer linear networks. The poor performance of the correlation receiver in this case is mainly due to the lack of any filtering of the received signal before the correlation decision stage. Selection of an optimal filter for this type of situation is not addressed in this thesis. Instead, the performance of the single layer linear network is assumed a fair representation of the performance of a correlation receiver using a nearly optimal filter chain to pre-process the received signal. The 2-layer and 3-layer networks performed equally well and both outperformed the single layer networks. 


\subsubsection{Training and Testing With Separate Noise Data}

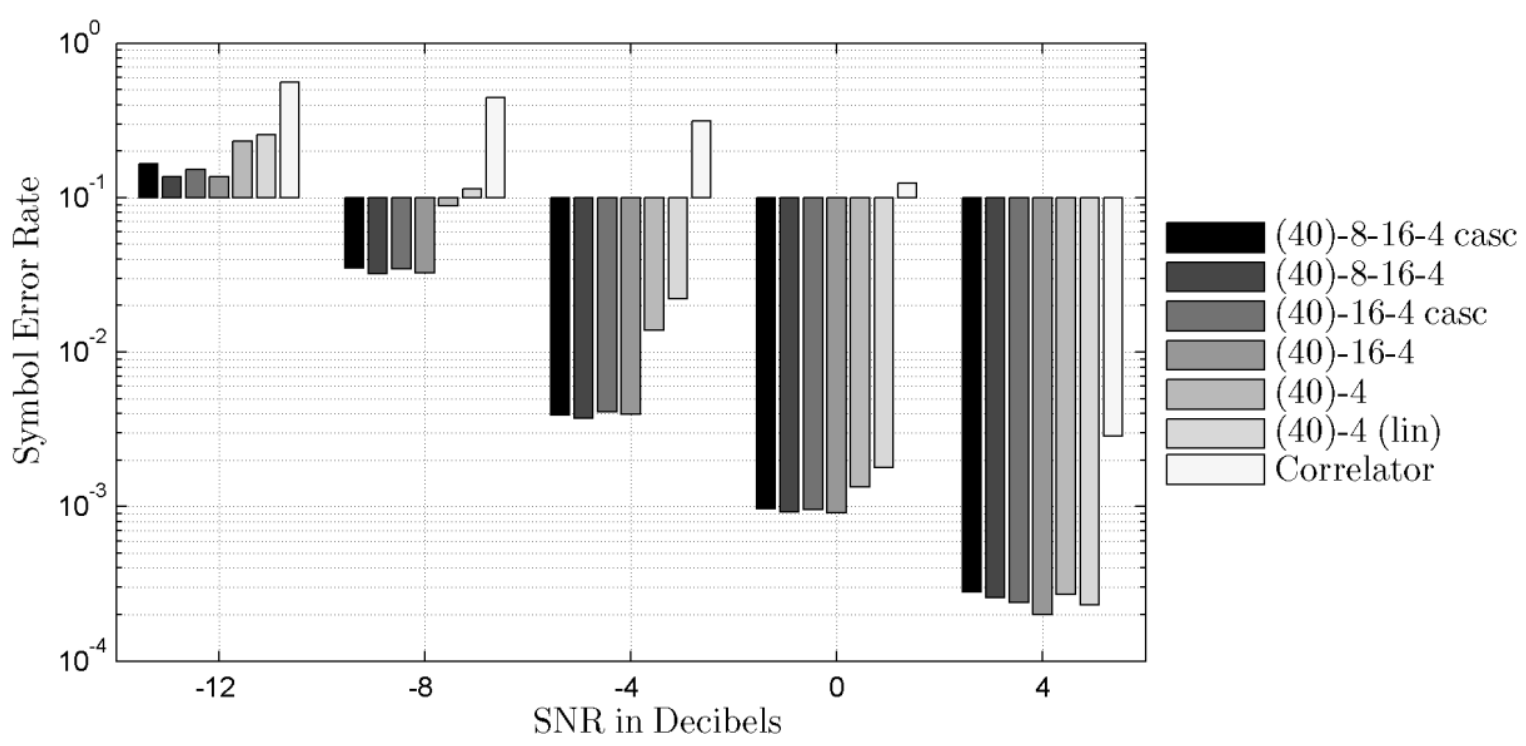

Figure 4-61 Separate Training and Test Noise with Field File \#1 and 4 Symbols

\begin{tabular}{|cllllc|}
\hline \multicolumn{7}{c|}{ SNR } \\
\hline Receiver & $\mathbf{- 1 2}$ & $\mathbf{- 8}$ & $\mathbf{- 4}$ & $\mathbf{0}$ & $\mathbf{4}$ \\
\hline$(40)-8-16-4$ casc & 0.16450 & 0.03500 & 0.00390 & 0.00100 & 0.00030 \\
$(40)-8-16-4$ & 0.13630 & 0.03210 & 0.00370 & 0.00090 & 0.00030 \\
$(40)-16-4$ casc & 0.15230 & 0.03440 & 0.00410 & 0.00100 & 0.00020 \\
$(40)-16-4$ & 0.13580 & 0.03250 & 0.00390 & 0.00090 & 0.00020 \\
$(40)-4$ & 0.23220 & 0.08880 & 0.01390 & 0.00130 & 0.00030 \\
$(40)-4$ (lin) & 0.25650 & 0.11390 & 0.02220 & 0.00180 & 0.00020 \\
Correlator & 0.56060 & 0.44560 & 0.31250 & 0.12360 & 0.00290 \\
\hline \# Symbols Tested & 84,000 & 100,000 & 100,000 & 100,000 & 100,000 \\
\hline
\end{tabular}

Table 4-51 SER Data for Figure 4-61

Figure 4-61 shows the same networks after being trained and tested using separate noise files. The neural network receivers are still clearly the best performers. 


\subsection{Simulations Using Field File \#2}
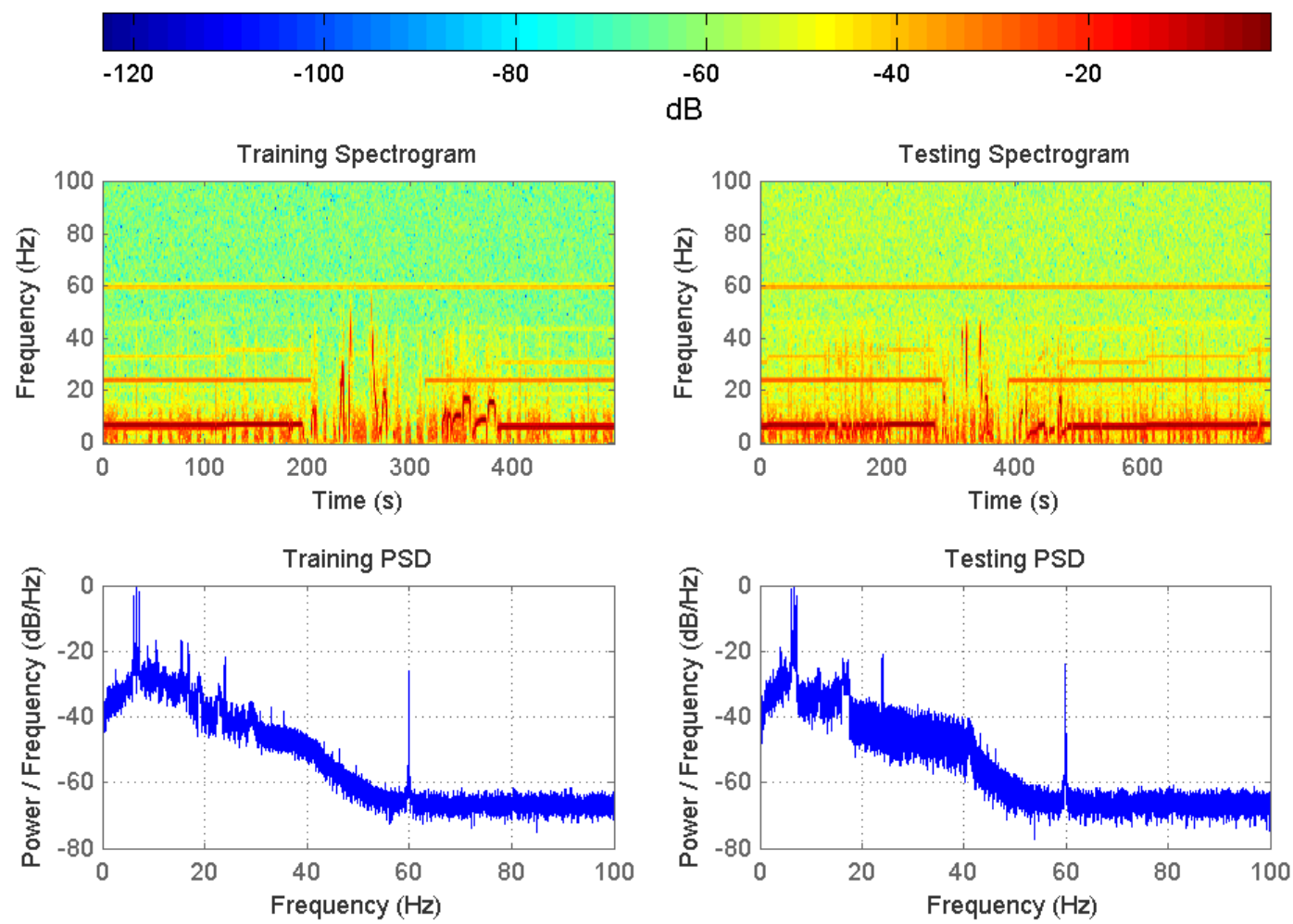

Figure 4-62 Frequency Content of Field File \#2. The power spectral density plots in the $2^{\text {nd }}$ row are generated from the exact same data as their respective spectrograms in the $1^{\text {st }}$ row.

Figure 4-62 shows the frequency content of Field File \#2. There are spectral peaks near $60 \mathrm{~Hz}, 24 \mathrm{~Hz}$, and $6 \mathrm{~Hz}$. There are visible steps in frequency in the 20 to $40 \mathrm{~Hz}$ band. The middle of the noise file differs from the rest of the file in both the training and test samples. There is also evidence of pulses from an EM MWD tool throughout the files. 


\subsubsection{Training and Testing With The Same Noise Data}

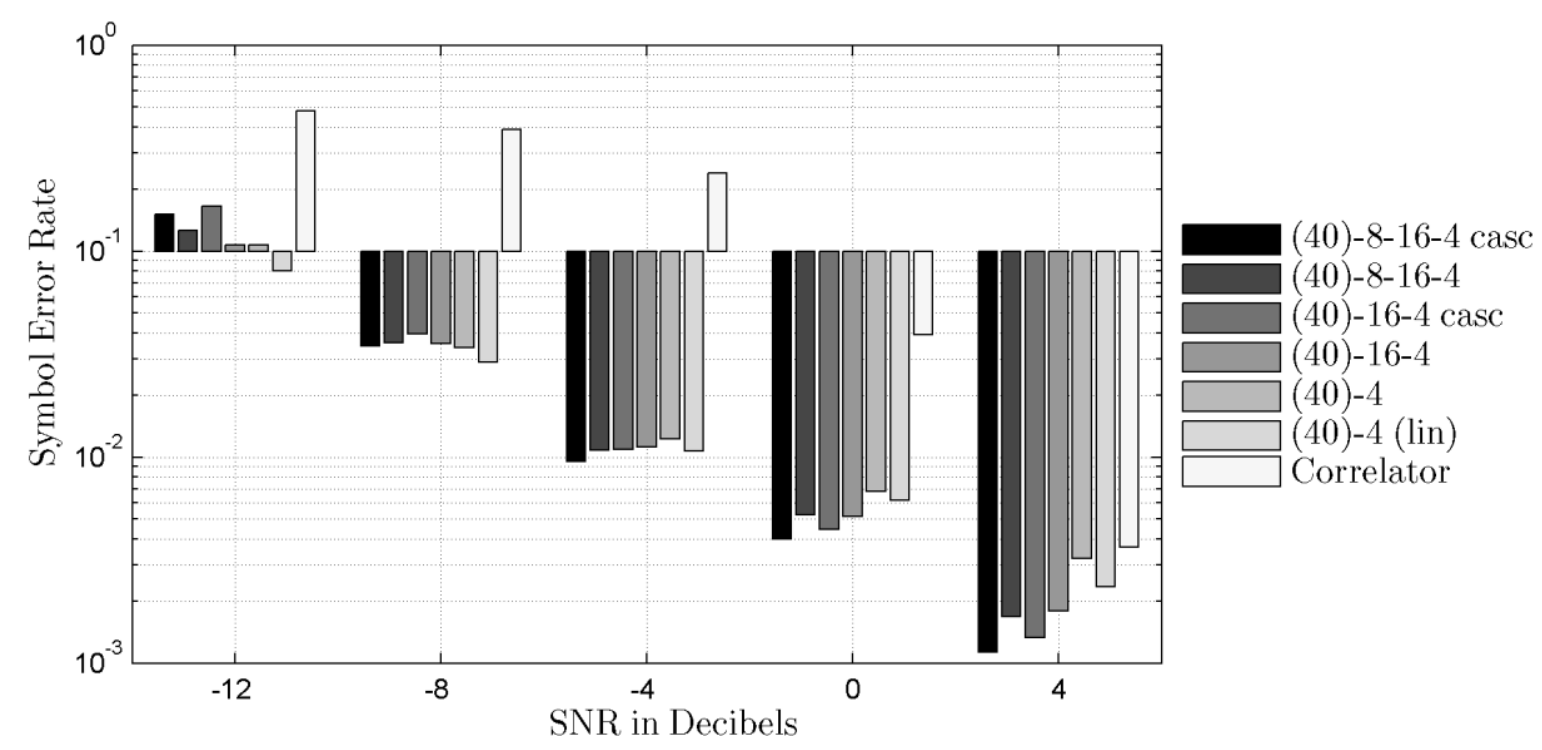

Figure 4-63 Same Training and Test Noise With Field File \#2 and 4 Symbols

\begin{tabular}{|cllllc|}
\multicolumn{7}{c|}{ SNR } \\
\hline Receiver & $\mathbf{- 1 2}$ & $\mathbf{- 8}$ & $\mathbf{- 4}$ & $\mathbf{0}$ & $\mathbf{4}$ \\
\hline (40)-8-16-4 casc & 0.15038 & 0.03464 & 0.00955 & 0.00399 & 0.00113 \\
$(40)-8-16-4$ & 0.12541 & 0.03574 & 0.01078 & 0.00523 & 0.00168 \\
$(40)-16-4$ casc & 0.16502 & 0.03981 & 0.01088 & 0.00446 & 0.00132 \\
$(40)-16-4$ & 0.10696 & 0.03568 & 0.01115 & 0.00514 & 0.00180 \\
$(40)-4$ & 0.10746 & 0.03413 & 0.01226 & 0.00684 & 0.00322 \\
(40)-4 (lin) & 0.08037 & 0.02898 & 0.01073 & 0.00616 & 0.00235 \\
Correlator & 0.47921 & 0.38862 & 0.23823 & 0.03937 & 0.00365 \\
\hline \# Symbols Tested & 100,000 & 100,000 & 100,000 & 100,000 & 100,000 \\
\hline
\end{tabular}

Table 4-52 SER Data for Figure 4-63

The neural network receivers in Figure 4-63 performed roughly the same as the sing-layer linear networks. 


\subsubsection{Training and Testing With Separate Noise Data}

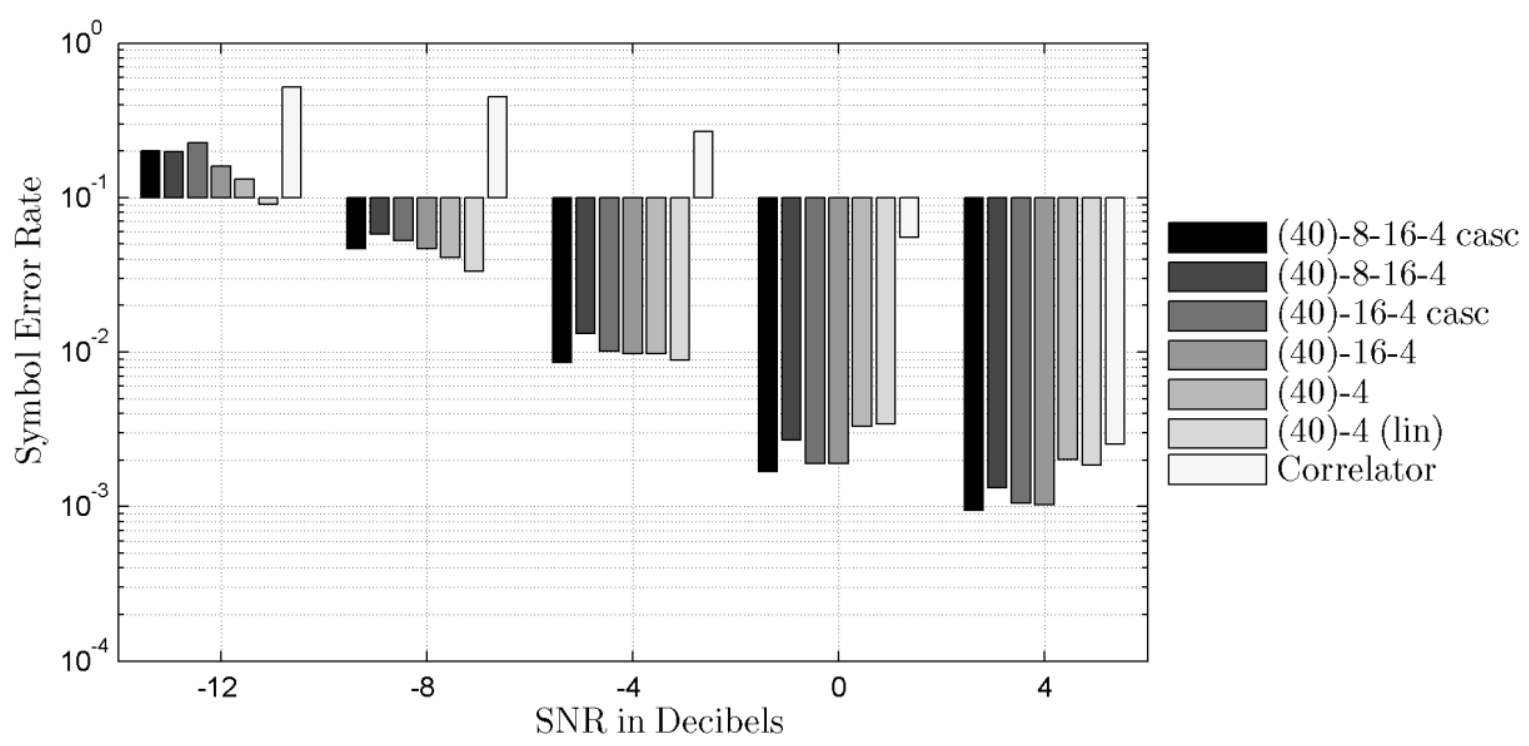

Figure 4-64 Separate Training and Test Noise with Field File \#2 and 4 Symbols

\begin{tabular}{|cllllc|}
\multicolumn{7}{c|}{ SNR } \\
\hline Receiver & $\mathbf{- 1 2}$ & $\mathbf{- 8}$ & $\mathbf{- 4}$ & $\mathbf{0}$ & $\mathbf{4}$ \\
\hline (40)-8-16-4 casc & 0.20047 & 0.04695 & 0.00859 & 0.00168 & 0.00095 \\
$(40)-8-16-4$ & 0.19765 & 0.05787 & 0.01314 & 0.00269 & 0.00133 \\
$(40)-16-4$ casc & 0.22532 & 0.05274 & 0.01012 & 0.00191 & 0.00106 \\
$(40)-16-4$ & 0.15974 & 0.04648 & 0.00978 & 0.00189 & 0.00103 \\
$(40)-4$ & 0.13179 & 0.04095 & 0.00980 & 0.00332 & 0.00201 \\
(40)-4 (lin) & 0.09024 & 0.03341 & 0.00885 & 0.00343 & 0.00184 \\
Correlator & 0.51990 & 0.44968 & 0.26830 & 0.05510 & 0.00253 \\
\hline \# Symbols Tested & 60,000 & 60,000 & 60,000 & 60,000 & 60,000 \\
\hline
\end{tabular}

Table 4-53 SER Data for Figure 4-64

The differences between Figure 4-63 and Figure 4-64 are minimal. 


\subsection{Simulations Using Field File \#3}
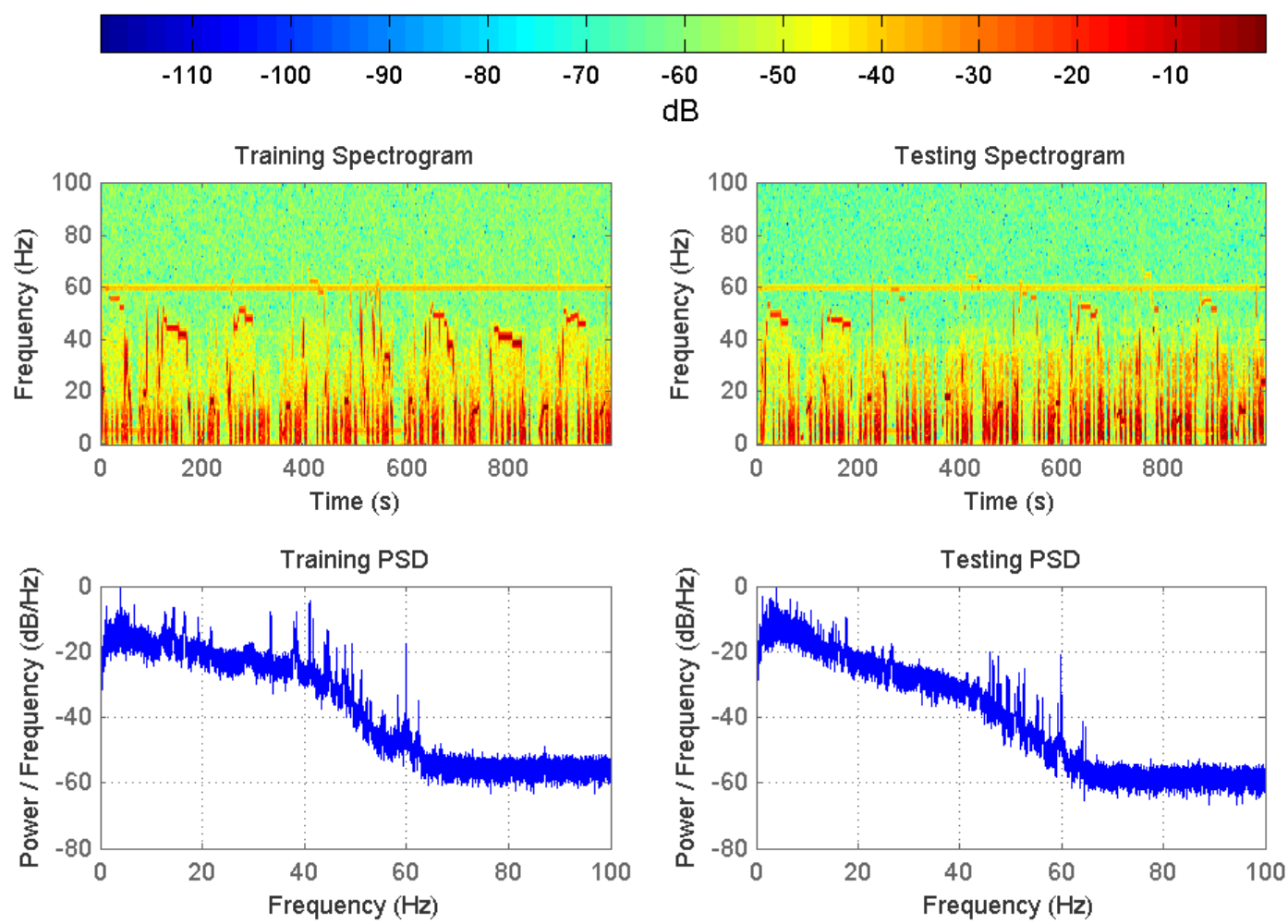

Figure 4-65 Frequency Content of Field File \#3. The power spectral density plots in the $2^{\text {nd }}$ row are generated from the exact same data as their respective spectrograms in the $1^{\text {st }}$ row.

Figure 4-65 shows the frequency content of Field File \#3. The spectrograms reveal a spectral peak at $60 \mathrm{~Hz}$ much like the other noise sources. There also appears to be the signal from another EM MWD tool in both the training and test portions of the noise file. The noise consisting of a ramp up in frequency, followed by a plateau, and ramp down in frequency, is most likely due to rig motor noise. 


\subsubsection{Training and Testing With The Same Noise Data}

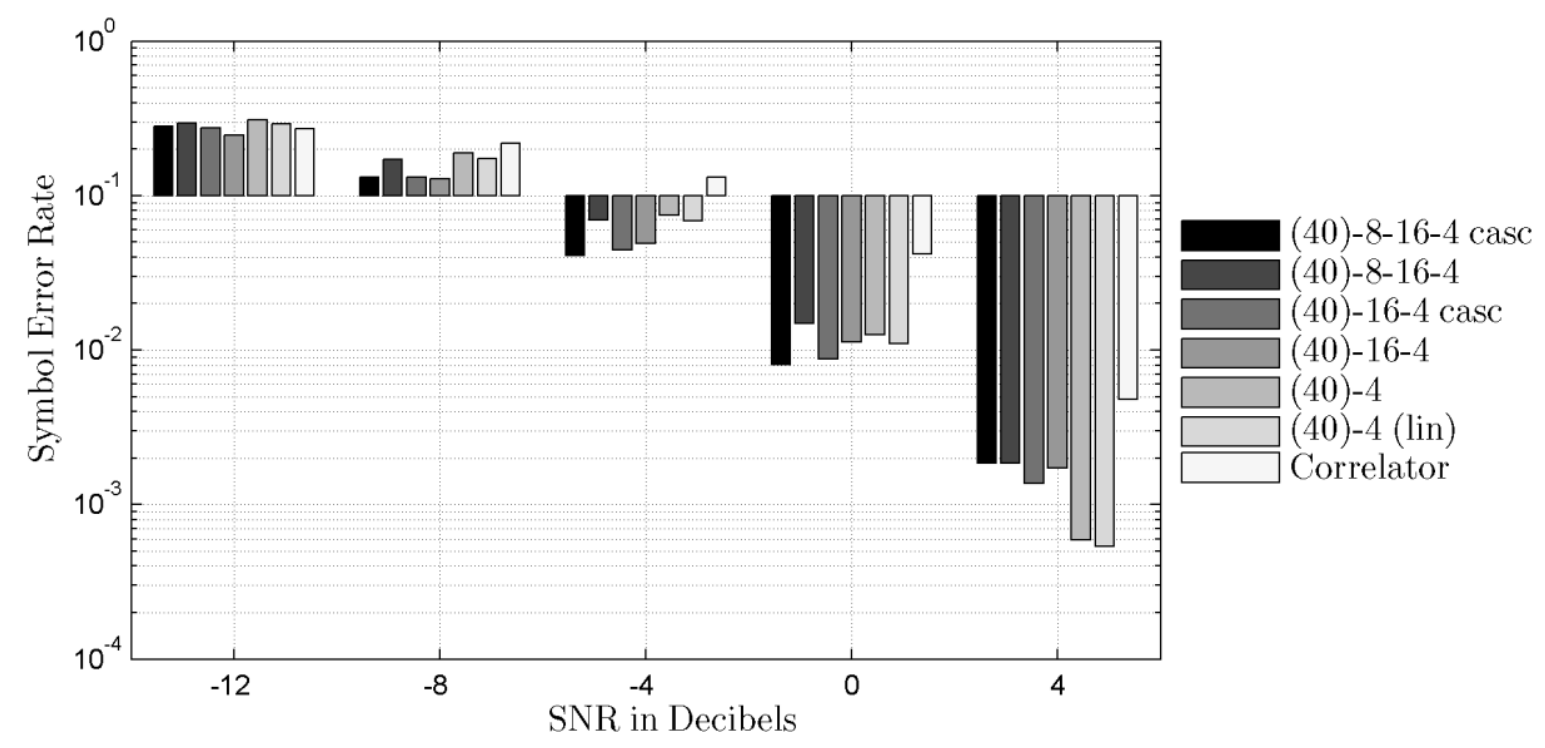

Figure 4-66 Same Training and Test Noise with Field File \#3 and 4 Symbols

\begin{tabular}{|cllllc|}
\hline \multicolumn{7}{c|}{ SNR } \\
\hline Receiver & $\mathbf{- 1 2}$ & $\mathbf{- 8}$ & $\mathbf{- 4}$ & $\mathbf{0}$ & $\mathbf{4}$ \\
\hline (40)-8-16-4 casc & 0.28077 & 0.13212 & 0.04106 & 0.00804 & 0.00186 \\
$(40)-8-16-4$ & 0.29337 & 0.17208 & 0.06922 & 0.01480 & 0.00186 \\
$(40)-16-4$ casc & 0.27407 & 0.13092 & 0.04442 & 0.00879 & 0.00138 \\
$(40)-16-4$ & 0.24590 & 0.12885 & 0.04925 & 0.01132 & 0.00172 \\
$(40)-4$ & 0.30770 & 0.18853 & 0.07438 & 0.01257 & 0.00059 \\
(40)-4 (lin) & 0.29152 & 0.17378 & 0.06834 & 0.01096 & 0.00053 \\
Correlator & 0.27157 & 0.21761 & 0.13087 & 0.04202 & 0.00482 \\
\hline \# Symbols Tested & 37,000 & 54,800 & 60,000 & 60,000 & 60,000 \\
\hline
\end{tabular}

Table 4-54 SER Data for Figure 4-66

The single layer linear network outperformed the neural networks when the SNR was greater than $0 \mathrm{~dB}$. The 2-layer network containing 16 neurons in the input layer performed the best when the SNR was less than $0 \mathrm{~dB}$. The differences in performance between the receivers were minimal except for when the SNR was $4 \mathrm{~dB}$. 


\subsubsection{Training and Testing With Separate Noise Data}

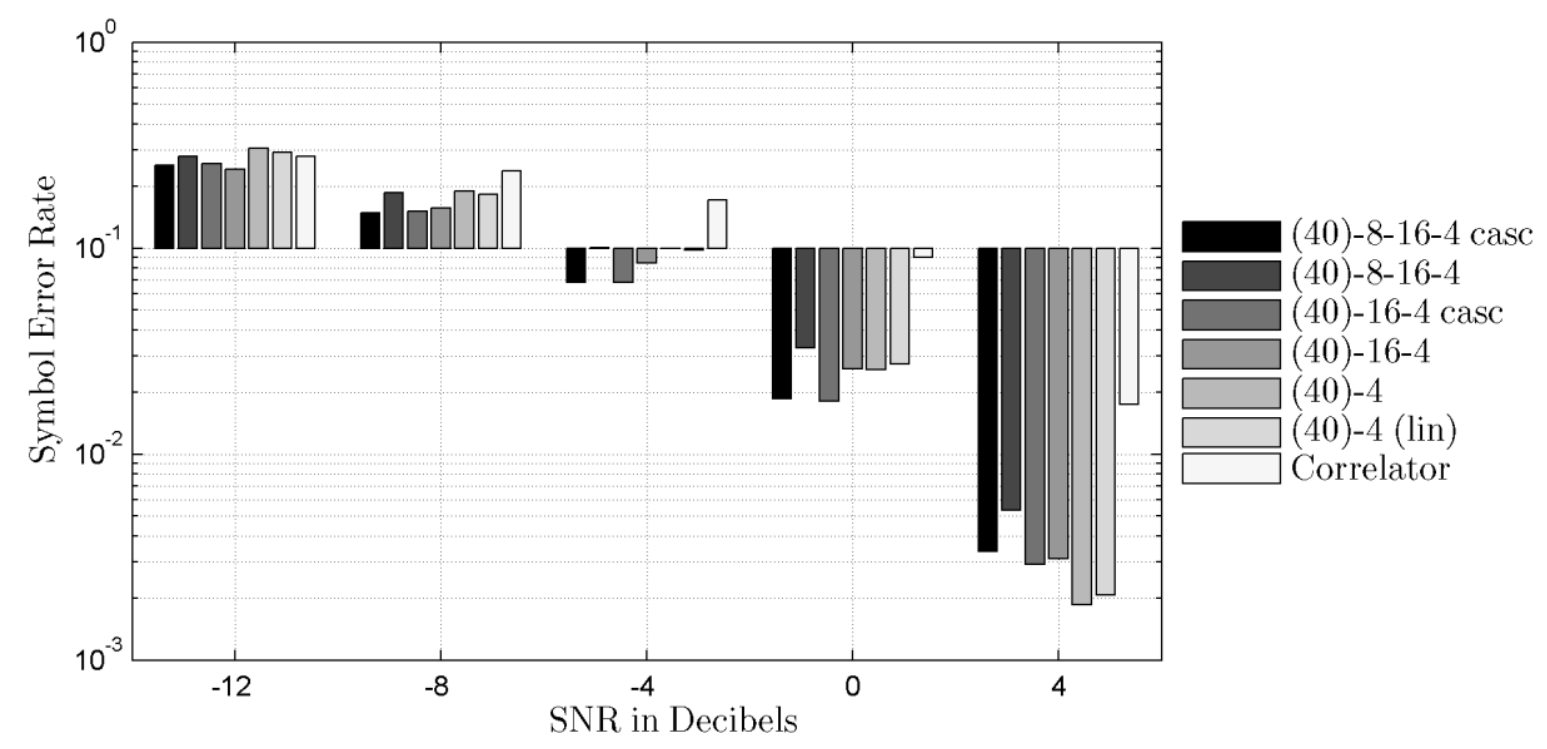

Figure 4-67 Separate Training and Test Noise with Field File \#3 and 4 Symbols

\begin{tabular}{|cclllc|}
\hline \multicolumn{7}{c}{ SNR } \\
\hline Receiver & $\mathbf{- 1 2}$ & $\mathbf{- 8}$ & $\mathbf{- 4}$ & $\mathbf{0}$ & $\mathbf{4}$ \\
\hline$(40)-8-16-4$ casc & 0.25183 & 0.14860 & 0.06783 & 0.01858 & 0.00338 \\
$(40)-8-16-4$ & 0.27786 & 0.18503 & 0.10033 & 0.03268 & 0.00536 \\
$(40)-16-4$ casc & 0.25797 & 0.15072 & 0.06792 & 0.01803 & 0.00293 \\
$(40)-16-4$ & 0.24220 & 0.15676 & 0.08483 & 0.02603 & 0.00312 \\
$(40)-4$ & 0.30548 & 0.18948 & 0.09920 & 0.02581 & 0.00185 \\
$(40)-4$ (lin) & 0.29142 & 0.18175 & 0.09769 & 0.02732 & 0.00207 \\
Correlator & 0.27855 & 0.23622 & 0.17045 & 0.08998 & 0.01738 \\
\hline \# Symbols Tested & 37,200 & 48,400 & 60,000 & 60,000 & 60,000 \\
\hline
\end{tabular}

Table 4-55 SER Data for Figure 4-67

The results shown in Figure 4-67 follow the performance of the previous simulation. 


\subsection{Simulations Using Field File \#4}
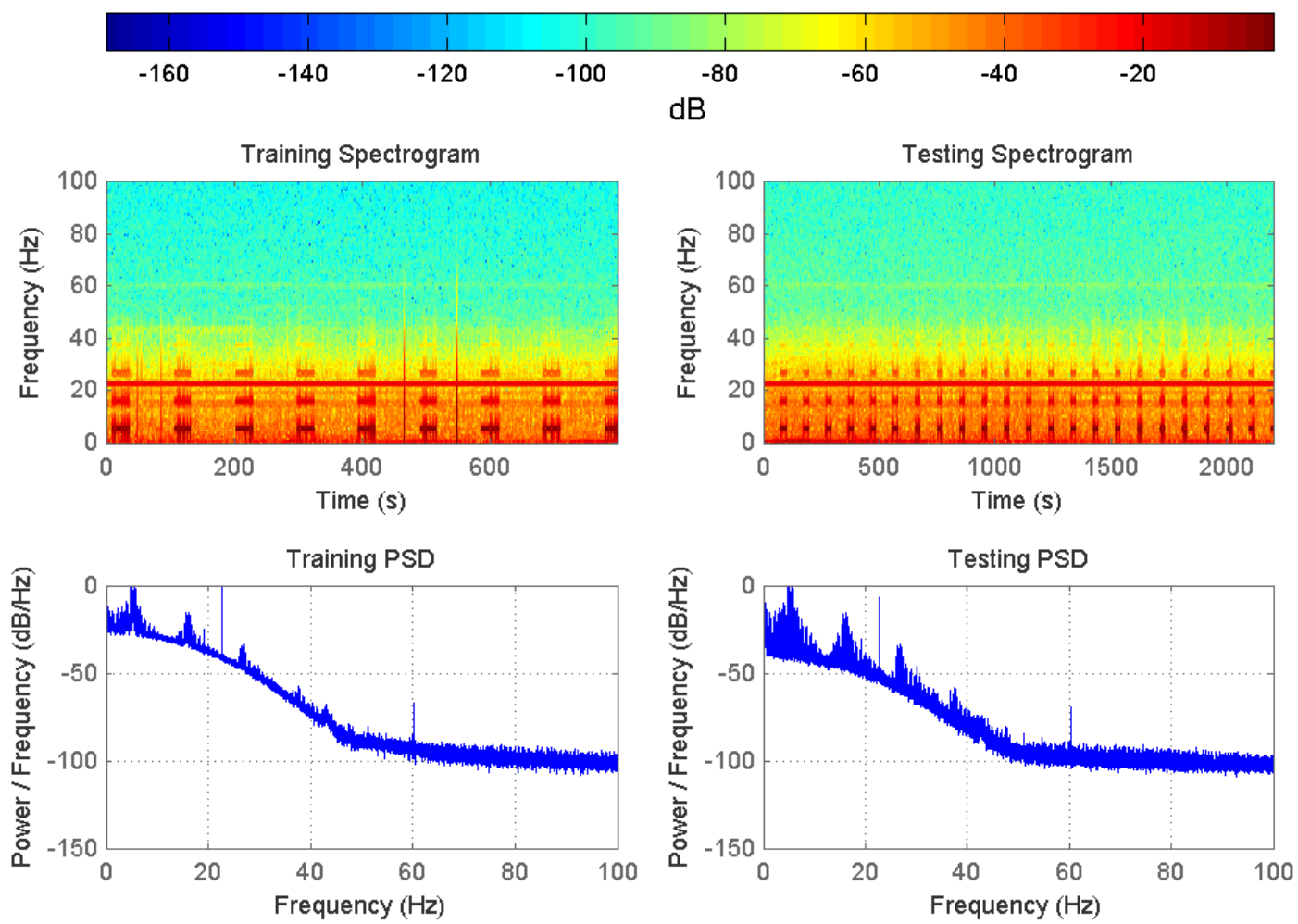

Figure 4-68 Frequency Content of Field File \#4. The power spectral density plots in the $2^{\text {nd }}$ row are generated from the exact same data as their respective spectrograms in the $1^{\text {st }}$ row.

The frequency content of Field File \#4 is shown in Figure 4-68. Some of the spectral components of the noise are similar to the previous files. There are peaks near $60 \mathrm{~Hz}$ and 22 Hz. Most of the energy is from a $5 \mathrm{~Hz}$ square wave. The power spectral density shows peaks at $5 \mathrm{~Hz}, 15 \mathrm{~Hz}$, and $25 \mathrm{~Hz}$ that are the odd harmonics of the square wave. 


\subsubsection{Training and Testing With the Same Noise Data}

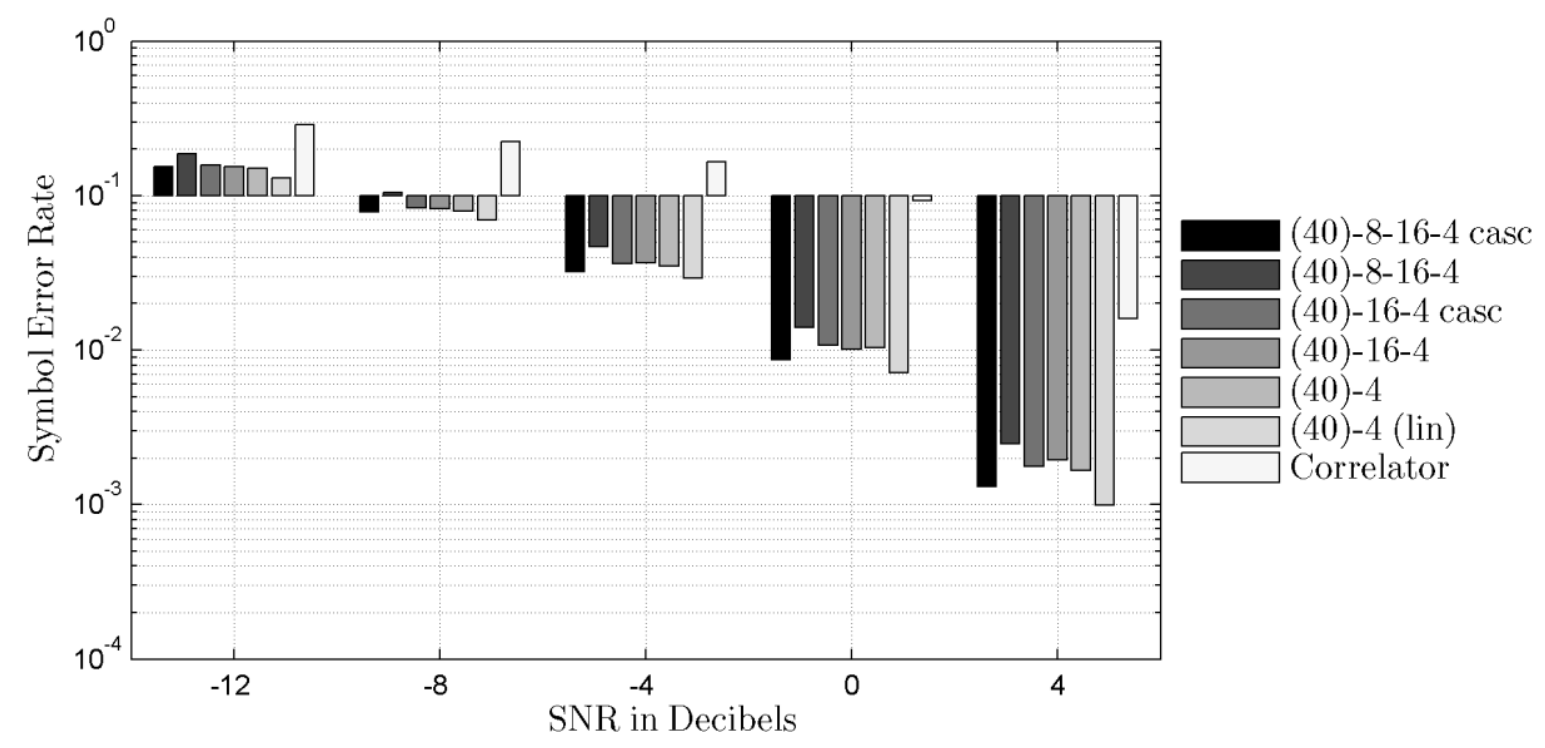

Figure 4-69 Same Training and Test Noise with Field File \#4 and 4 Symbols

\begin{tabular}{|cllllc|}
\hline \multicolumn{7}{c|}{ SNR } \\
\hline Receiver & $\mathbf{- 1 2}$ & $\mathbf{- 8}$ & $\mathbf{- 4}$ & $\mathbf{0}$ & $\mathbf{4}$ \\
\hline (40)-8-16-4 casc & 0.15341 & 0.07842 & 0.03224 & 0.00863 & 0.00130 \\
$(40)-8-16-4$ & 0.18638 & 0.10467 & 0.04652 & 0.01405 & 0.00247 \\
$(40)-16-4$ casc & 0.15829 & 0.08372 & 0.03647 & 0.01073 & 0.00176 \\
$(40)-16-4$ & 0.15292 & 0.08238 & 0.03682 & 0.01012 & 0.00195 \\
$(40)-4$ & 0.14928 & 0.07978 & 0.03482 & 0.01040 & 0.00166 \\
(40)-4 (lin) & 0.13051 & 0.06938 & 0.02917 & 0.00710 & 0.00099 \\
Correlator & 0.28711 & 0.22459 & 0.16529 & 0.09307 & 0.01608 \\
\hline \# Symbols Tested & 84,400 & 100,000 & 100,000 & 100,000 & 100,000 \\
\hline
\end{tabular}

Table 4-56 SER Data for Figure 4-69

The single-layer linear network performed the best for this noise file. The non-linear single layer network and the 2-layer networks performed equally well. 


\subsubsection{Training and Testing With Separate Noise Data}

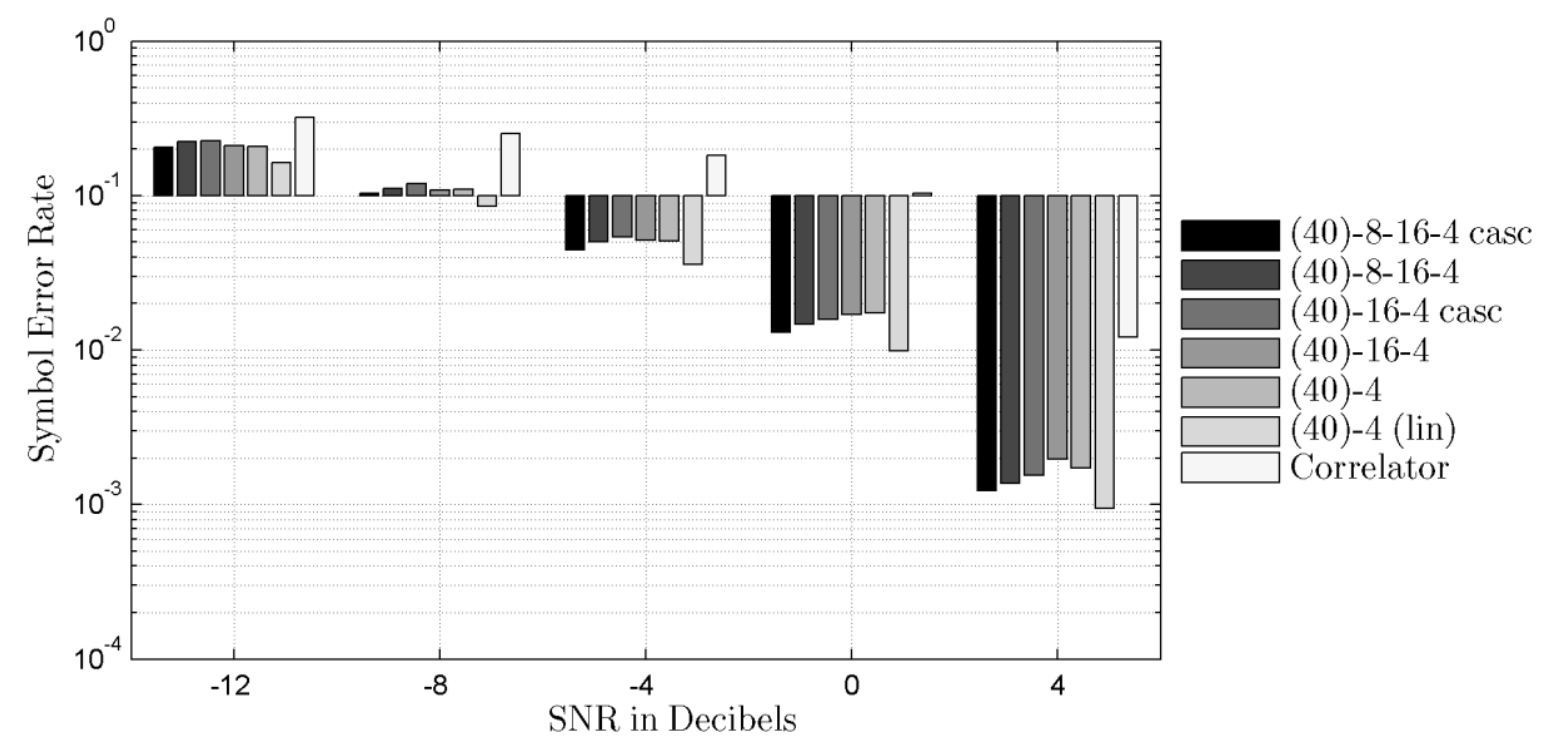

Figure 4-70 Separate Training and Test Noise with Field File \#4 and 4 Symbols

\begin{tabular}{|cllllc|}
\multicolumn{7}{c|}{ SNR } \\
\hline Receiver & $\mathbf{- 1 2}$ & $\mathbf{- 8}$ & $\mathbf{- 4}$ & $\mathbf{0}$ & $\mathbf{4}$ \\
\hline (40)-8-16-4 casc & 0.20527 & 0.10276 & 0.04462 & 0.01308 & 0.00124 \\
$(40)-8-16-4$ & 0.22328 & 0.11173 & 0.05034 & 0.01468 & 0.00137 \\
$(40)-16-4$ casc & 0.22706 & 0.11921 & 0.05387 & 0.01573 & 0.00155 \\
$(40)-16-4$ & 0.21065 & 0.10905 & 0.05135 & 0.01700 & 0.00197 \\
$(40)-4$ & 0.20868 & 0.10994 & 0.05096 & 0.01750 & 0.00172 \\
(40)-4 (lin) & 0.16247 & 0.08531 & 0.03563 & 0.00993 & 0.00094 \\
Correlator & 0.31992 & 0.25179 & 0.18155 & 0.10319 & 0.01213 \\
\hline \# Symbols Tested & 76,800 & 100,000 & 100,000 & 100,000 & 100,000 \\
\hline
\end{tabular}

Table 4-57 SER Data for Figure 4-70

The performance of the networks is almost identical to the performance shown in Figure 4-69. 


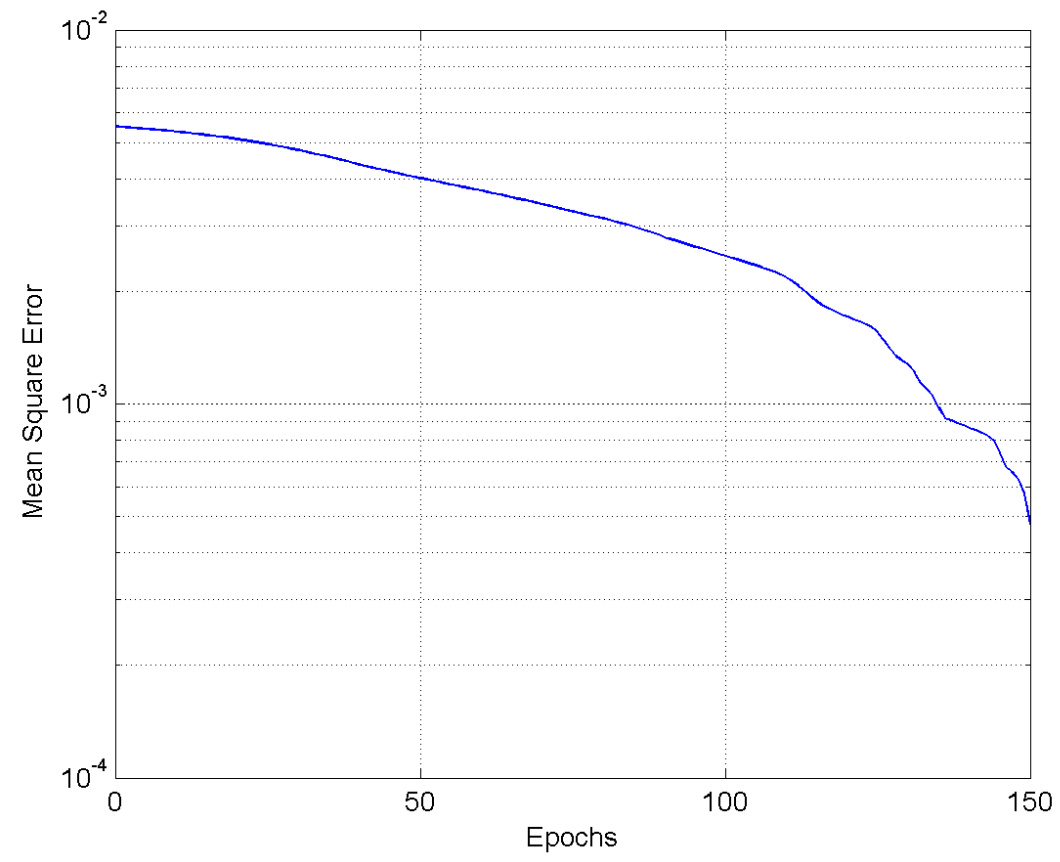

Figure 4-71 - Mean squared error performance during training of 3-layer cascade network

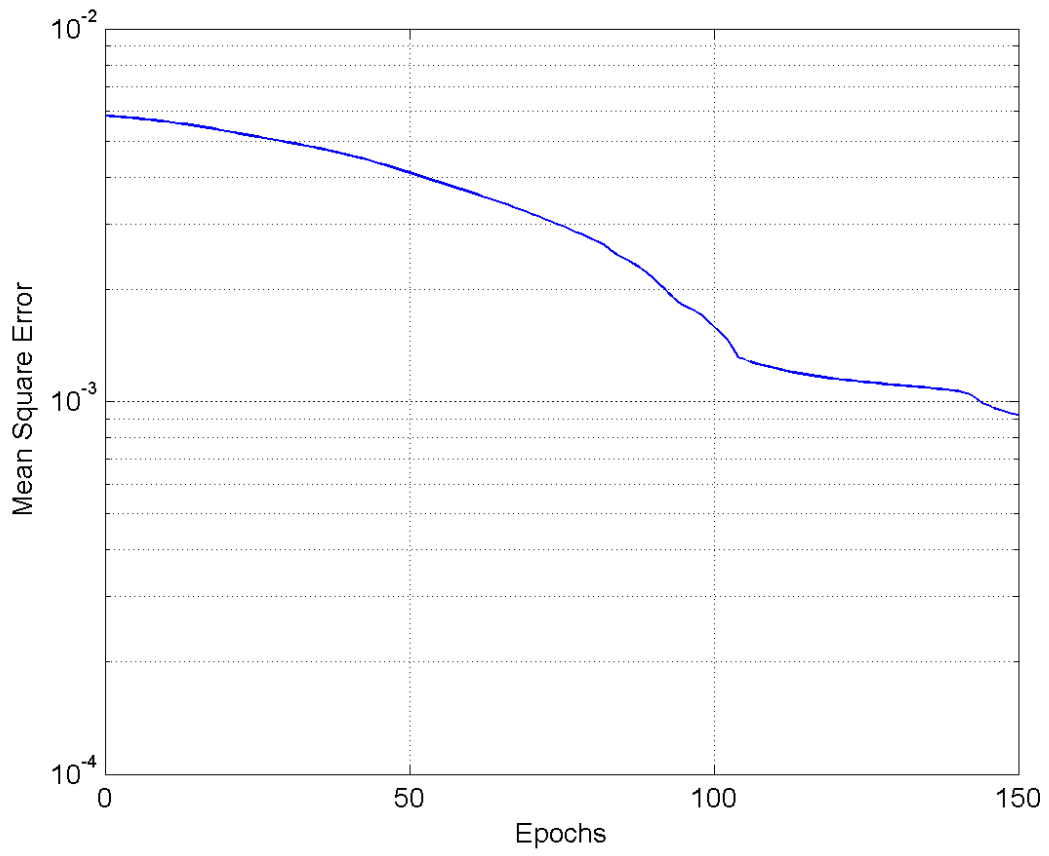

Figure 4-72 - Mean squared error performance during training of 3-layer feed forward network 
Figure 4-71 and Figure 4-72 show examples of the mean squared error performance during training of two network receivers. Each point of the plots is the mean squared error of the given network taken over the entire training batch.

\subsection{Summary of Simulations and Results}

The neural network receivers containing a single layer of linear neurons performed equally as well as a correlation receiver in the presence of AWGN. The resulting trained weights of the networks were shown to closely resemble the prototype waveforms used by the correlation receiver. This should be expected, as the architecture of such a network is the same as a correlation receiver. The nonlinear networks and the multilayer networks performed slightly worse than the correlation receiver in the presence of AWGN.

Tests performed with $1 / \mathrm{f}$ noise resulted in the neural networks outperforming the correlation receiver. The gap in performance between the ANN receivers and the correlation receiver grew as the noise color went from white to pink and beyond. It should be noted that knowledge of the statistical characteristics of the noise encountered at a drill site would allow for the implementation of optimal filtering for a correlation receiver. The simple correlation receiver implementation used did not attempt to find the optimal filtering for each noise source. However, the optimal filtering and correlation receiver combination is expected to perform as well as, if not slightly better, than the trained linear neural network receivers that were tested. The advantage of the ANN receivers is lack of expert knowledge required to achieve near-optimal performance. The nonlinear networks clearly outperformed the linear networks as the noise deviated further and further from AWGN. It is not known how their 
performance would compare to that of a correlation receiver proceeded by an optimal filtering chain. This would require further testing and is left as possible future work.

The ANN receivers outperformed the correlation receiver when using sampled data taken from actual drilling sites. In these cases, the non-linear multilayer networks noticeably outperformed the correlation receiver and the linear single layer networks.

The performance of the networks decreased significantly as the number of concurrent input nodes was reduced. This makes intuitive sense as increasing the number of inputs essentially provides the network with more information with which to base its decision on. With a reduced number of inputs, the sequential outputs must be post-processed to determine a single decision for the data of a received symbol. The post processing was simply a weighted sum of each sequential output for each output neuron. Modifying the weights of the post processing method from a flat average to one that more closely matched the filtering of the channel resulted in some improvement when the number of input nodes was less than the number of sampled data points per symbol.

The inclusion of recurrent connections did not improve the performance of the resulting trained networks. Testing of the recurrent networks required significantly more processing time and thus only a few initial tests were performed.

The effect of variations on both the number of layers and the size of each layer were observed. The network consisting of only a single layer performed the best when dealing with AWGN, which was expected. Increasing the number of layers from 2 to 3 did not result in a noticeable improvement in performance. The multilayer networks performed better than the single layer networks in the presence of non-white noise. 


\section{Chapter 5 Conclusions and Future Work}

The viability of using neural networks as digital communication receivers in the EM MWD environment was confirmed by the simulation results. The performance of the trained neural networks showed improvements over a simple correlation receiver in some cases. As was predicted, the neural network receivers outperformed a correlation receiver in situations where the noise source was not AWGN.

The similarities between a correlation receiver and the neural network receivers are observed in this thesis. This was evident after looking at the resulting weights of a single layer linear network after being trained in the presence of AWGN. The weights closely match the prototype waveforms of the correlation receiver.

Utilizing the ANN receivers of this thesis in an actual EM MWD communication system would require more testing. The number of symbols in the code set of a real system could be well over 100, whereas the code sets tested in this thesis contained 4 or 8 symbols. The number of connections would increase dramatically as the size of the code set increases, which results in an increase in the processing time for the network. The method of training the network would also have to be addressed in the light of actual operation. It would not be practical to have the EM MWD tool send every symbol in the code set for use in adapting the weights of the receiver. The battery power wasted at the transmitter would negate the increased depth potential of an improved receiver. Nevertheless, the applications of ANN in EM MWD communication systems yield great potentials and warrants further study. The following list contains ideas for future work that others may pursue in this field. 
1. Address the problem of digital communication synchronization in the EM MWD environment using neural networks. This thesis assumed that the ANN receiver was synchronized with the transmitter, meaning it had knowledge of the exact start and stop times of each symbol being transmitted. Further work could explore methods of obtaining the correct start and stop times as well as the reduction in performance when the transmitter and receiver are slightly out of synchronization.

2. Evaluate other neural network architectures, learning algorithms, as well as growing and pruning options, such as cascade correlation.

3. Evaluate the performance of neural network receivers with a variety of different code sets.

4. Train the ANN receiver to recognize all codes when only certain codes are being transmitted in a bounded time window.

5. Implement adaptive noise cancellation in the EM MWD environment using neural networks.

6. Apply neural networks to the problem of Mud-pulse MWD communication reception. The noise produced by the mud pumps as well as pulse reflections in the drilling fluid are potential problems that could be addressed with neural networks. Each of these problems limits the performance of Mud-pulse communication. 


\section{Chapter 6 References}

[1] White, M.A. Adaptive Signaling for Sub-Surface Telemetry Systems. Thesis. California Polytechnic. San Luis Obispo, 2004.

[2] Rigzone. “How Does Measurement-While-Drilling (MWD) Work?” viewed 01-20-10 <http://www.rigzone.com/training/insight.asp?i_id=296>

[3] Suh, A.E. Noise Cancellation in Electromagnetic Measurement While Drilling Using Spectral Subtraction. Thesis. California Polytechnic. San Luis Obispo, 2004.

[4] Presco. "Measurement While Drilling." viewed 02-08-11, <http://www.prescoinc.com/science/drilling.htm $>$

[5] Sklar, B. Digital Communications: Fundamentals and Applications, P T R Prentice Hall, 1988.

[6] Cummins, F. and Schraudolph, N. "Introduction to Neural Networks", viewed 05-21-08, <http://cspeech.ucd.ie/ fred/teaching/oldcourses/ann98/intro.html>

[7] Brown, L.D. "Interval Estimation for a Binomial Proportion" Statistical Science Vol. 16, No.2 (2001): 101-133.

[8] Anderson, D. and McNeil, G. “Artificial Neural Networks Technology”, viewed 05-21-08, <https://www.dacs.dtic.mil/techs/neural/>

[9] Ritchison, G. "Human Physiology - Neurons \& the Nervous System", viewed 05-21-08, <http://people.eku.edu/ritchisong/301 notes2.htm>

[10] Orme, F. “The Action Potential and Nerves", viewed 05-21-08, $\langle\underline{\text { http://members.aol.com/Bio50/LecNotes/lecnot11.html }>}$ 
[11] Hagan, M. T. and M. Menhaj, "Training feedforward networks with the Marquardt algorithm." IEEE Transactions on Neural Networks, vol. 5, no. 6, 1994: 989-993.

[12] Lippert, R. A. "Introduction to Non-Linear Optimization", viewed 09-07-10, <http://www.mit.edu/ 9.520/spring08/Classes/optlecture.pdf $>$

[13] De Jesus, O. and Hagan, M.T. "Backpropagation Algorithms for a Broad Class of Dynamic Networks.” IEEE Transactions on Neural Networks Vol. 18, No. 1 (January 2007): 14-27.

[14] Orr, G. "Neural Networks", viewed 05-21-08, <http://www.willamette.edu/ gorr/classes/cs449/intro.html>

[15] Doya, K. "Recurrent Networks: Learning Algorithms.” Kyoto, 2002.

[16] Gorodnichy, D. “Associative Neural Networks as Means for Low-Resolution VideoBased Recognition.” International Joint Conference on Neural Networks. Montreal: National Research Council of Canada, 2005, NRC 48217.

[17] Fernandes, M.A.C., Neto, A.D.D. and Bezerra, J.B. “A Neural Network Model Applied to the Detection of Digital Signals.” IEEE, August 1998: 279-283.

[18] Proakis, J.G. and Manolakis, D.G. Digital Signal Processing: Principles, Algorithms and Applications, 3rd Edition, Prentice Hall, 1996.

[19] Haykin, S. “Adaptive Digital Communication Receivers.” IEEE Communications Magazine, December 2000: 106-114.

[20] Pearlmutter, B. A. "Gradient calculations for dynamic recurrent neural networks: A survey." IEEE Transactions on Neural Networks, vol. 6, 1995: 1212-1228.

[21] Williams, R. J. and Zipser D. “A learning algorithm for continually running fully recurrent neural networks," Neural Computation, vol. 1, no. 2, 1989: 270-280. 
[22] Trofimenkoff, F N, et al. "Characterization of EM Downhole-to-Surface Communication Links." IEEE Transactions on Geoscience and Remote Sensing, vol. 38, no. 6, November 2000.

[23] Wait, J. R., and Hill, D. A. "Theory of transmission of electromagnetic waves along a drill string rod in conducting rock", IEEE Transactions on Geoscience Electronics, vol. 17 , no. 2, 1979: 21-24. 


\section{Appendix A Source Code}

All simulations of this thesis used Matlab version 7.0.1.15 (R14) with the neural network toolbox. A script file was written for each simulation in order to configure the architectures of the networks, the training parameters, and the test parameters. Only a few selected script files are shown in this appendix as all the script files are of similar structure.

The functions for generating noise, testing networks, and training networks are included along with miscellaneous functions for processing and viewing the results.

\section{A.1 Noise Generation Source Code}

\section{A.1.1 GenerateNoiseDB2.m}

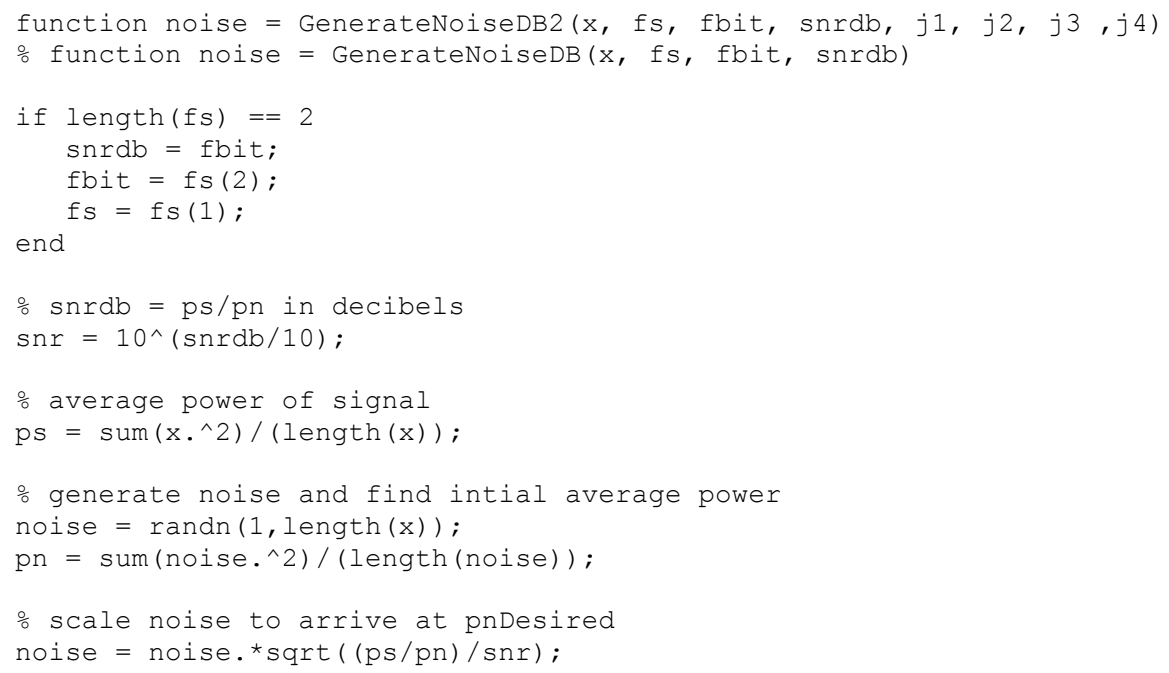

\section{A.1.2 GenerateNoiseBetaShaped.m}

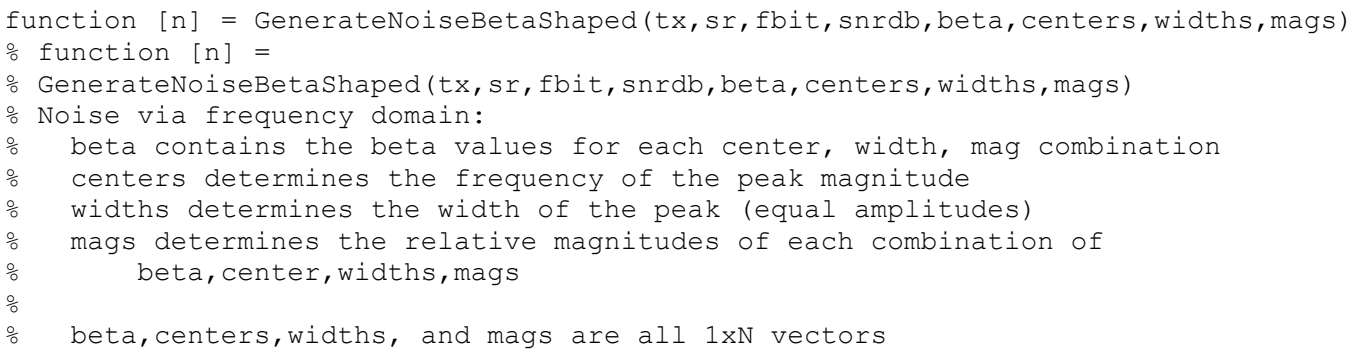




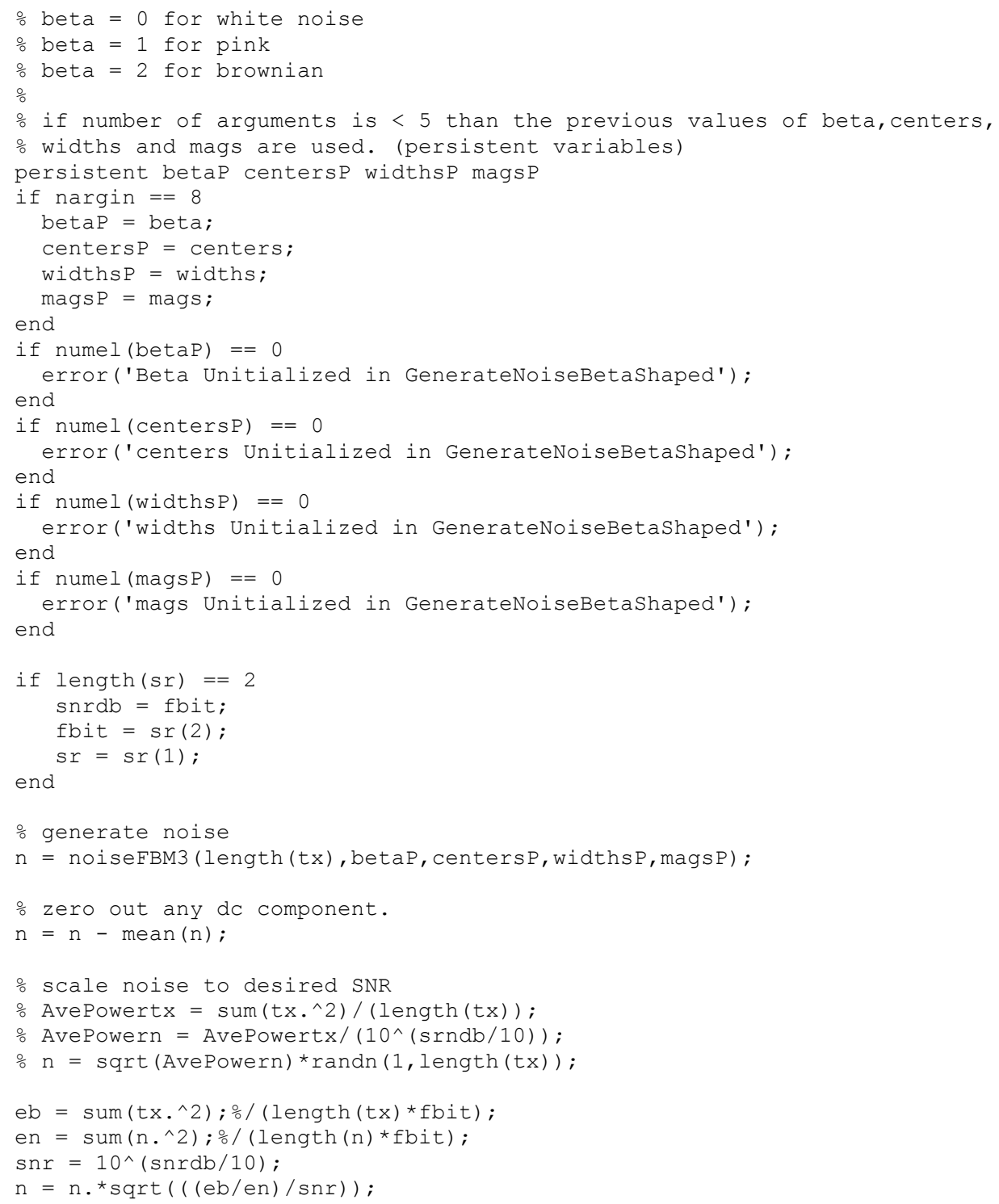

\section{A.1.3 NoiseFBM3.m}

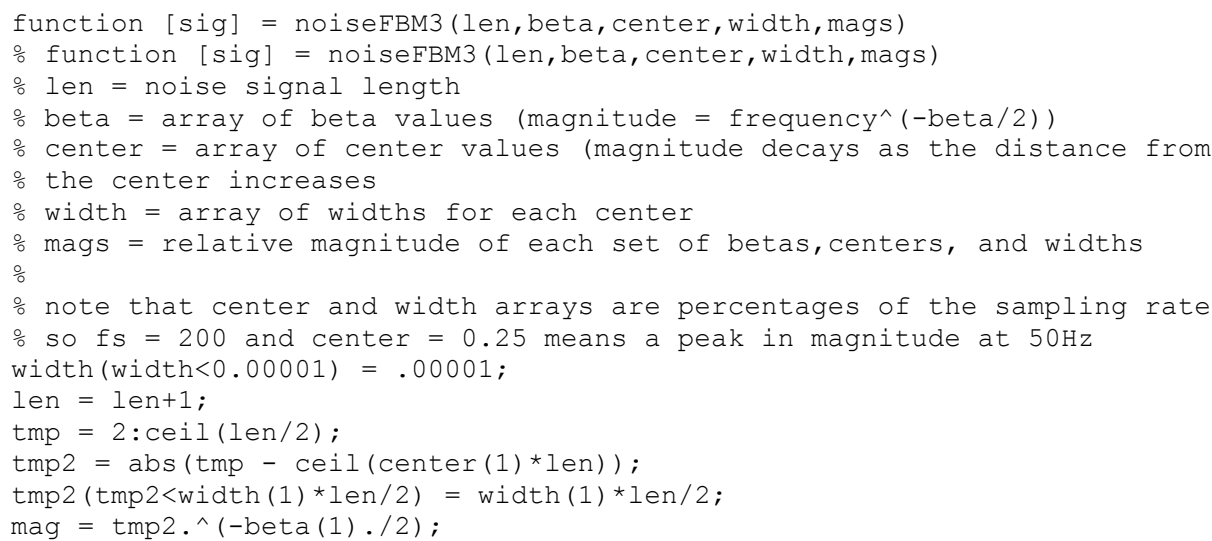




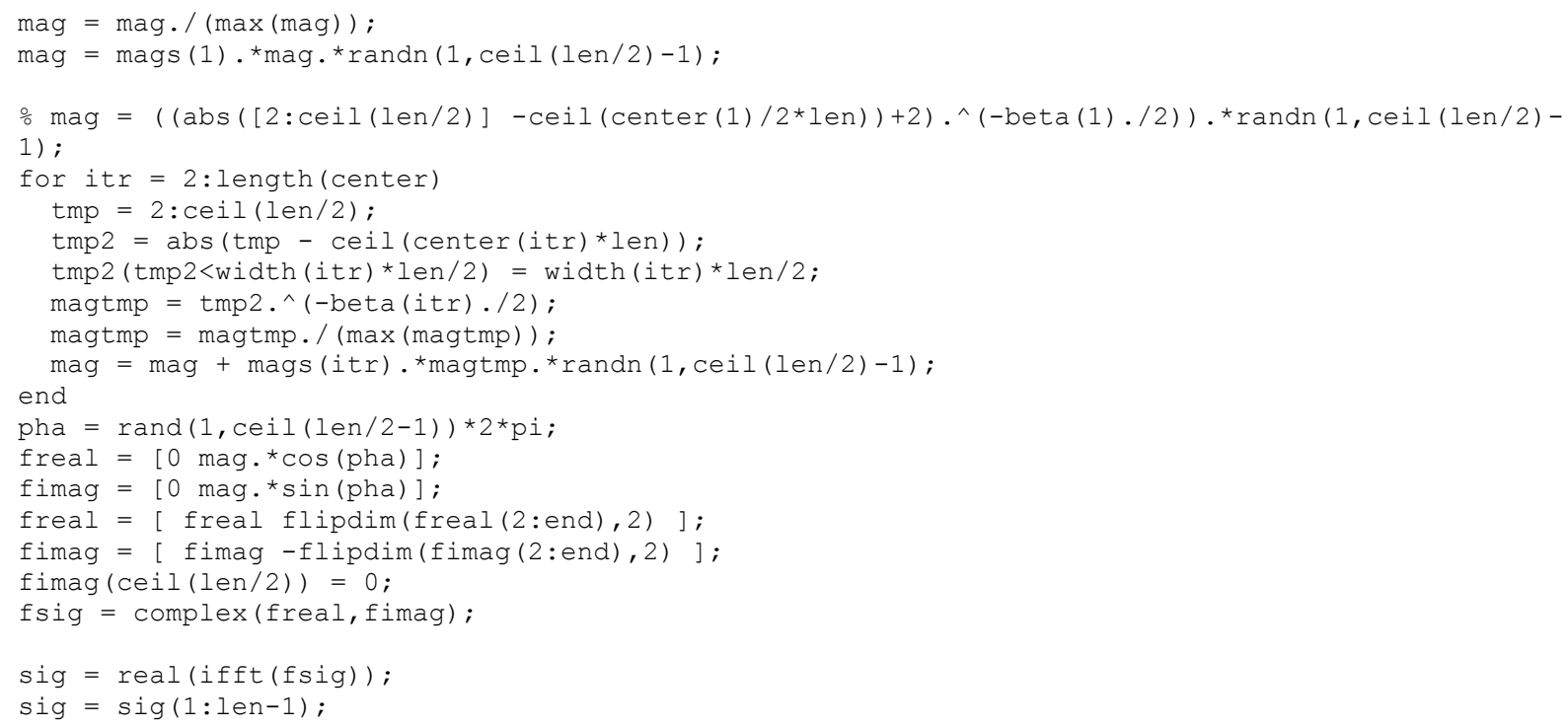

\section{A.1.4 getNoiseFromFile3.m}

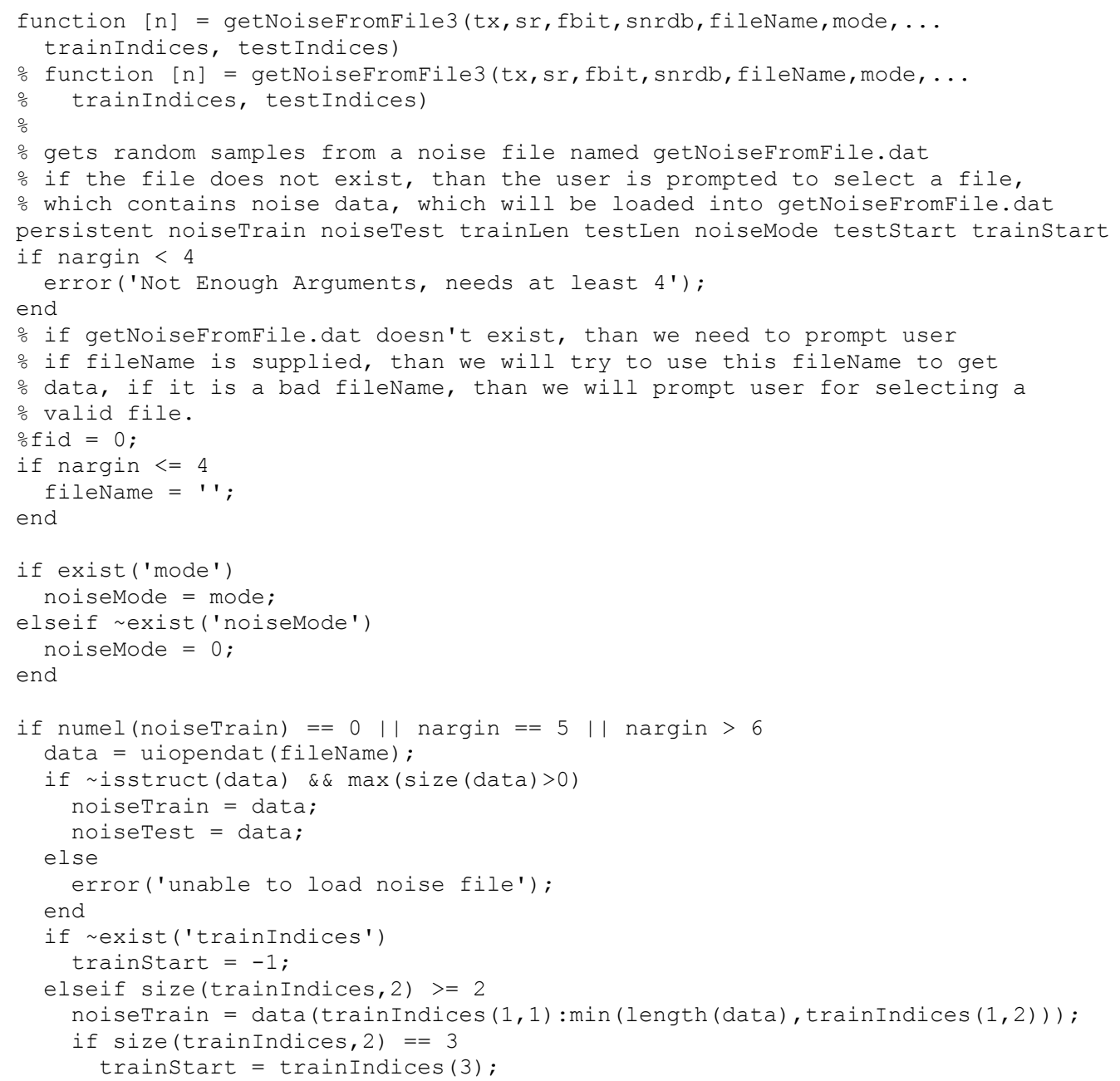




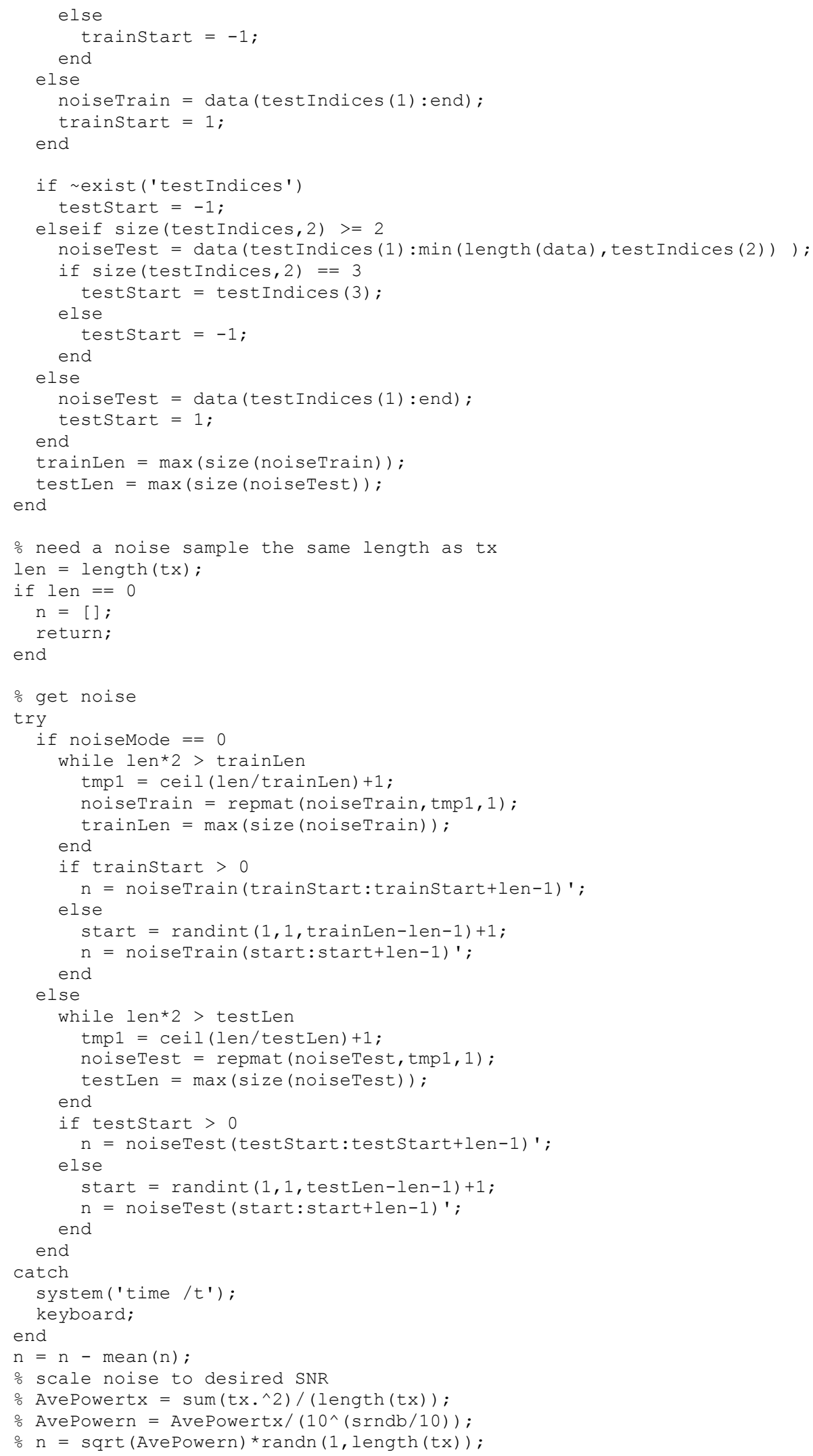




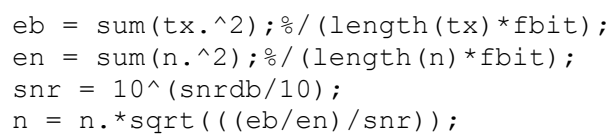

\section{A.2 Correlation Receiver Source Code}

\section{A.2.1 corrReceiver.m}

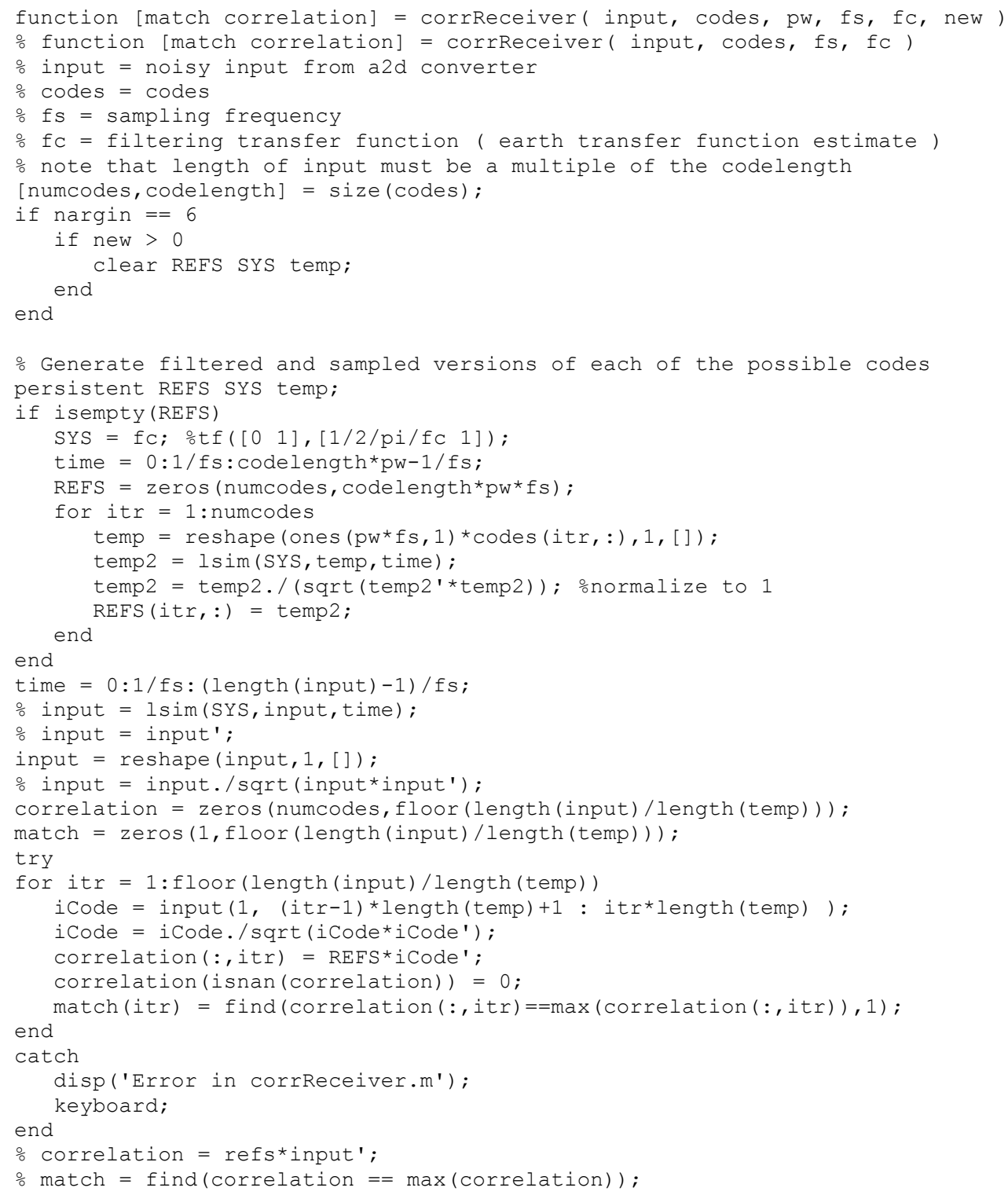




\section{A.3 Support Functions Source Code}

\section{A.3.1 GenerateOrthogonalPatterns.m}

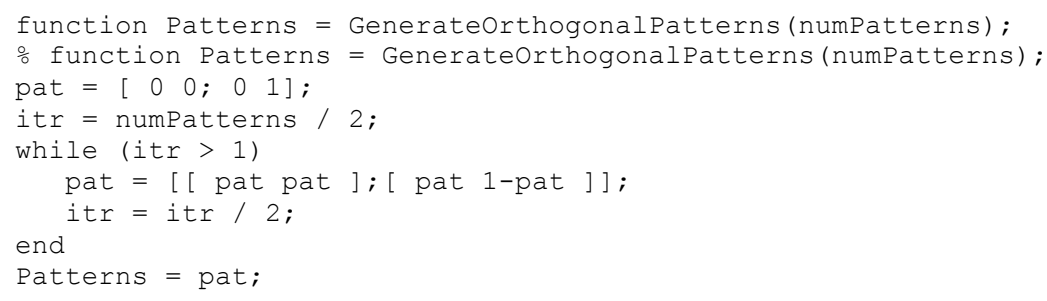

\section{A.3.2 genSig.m}

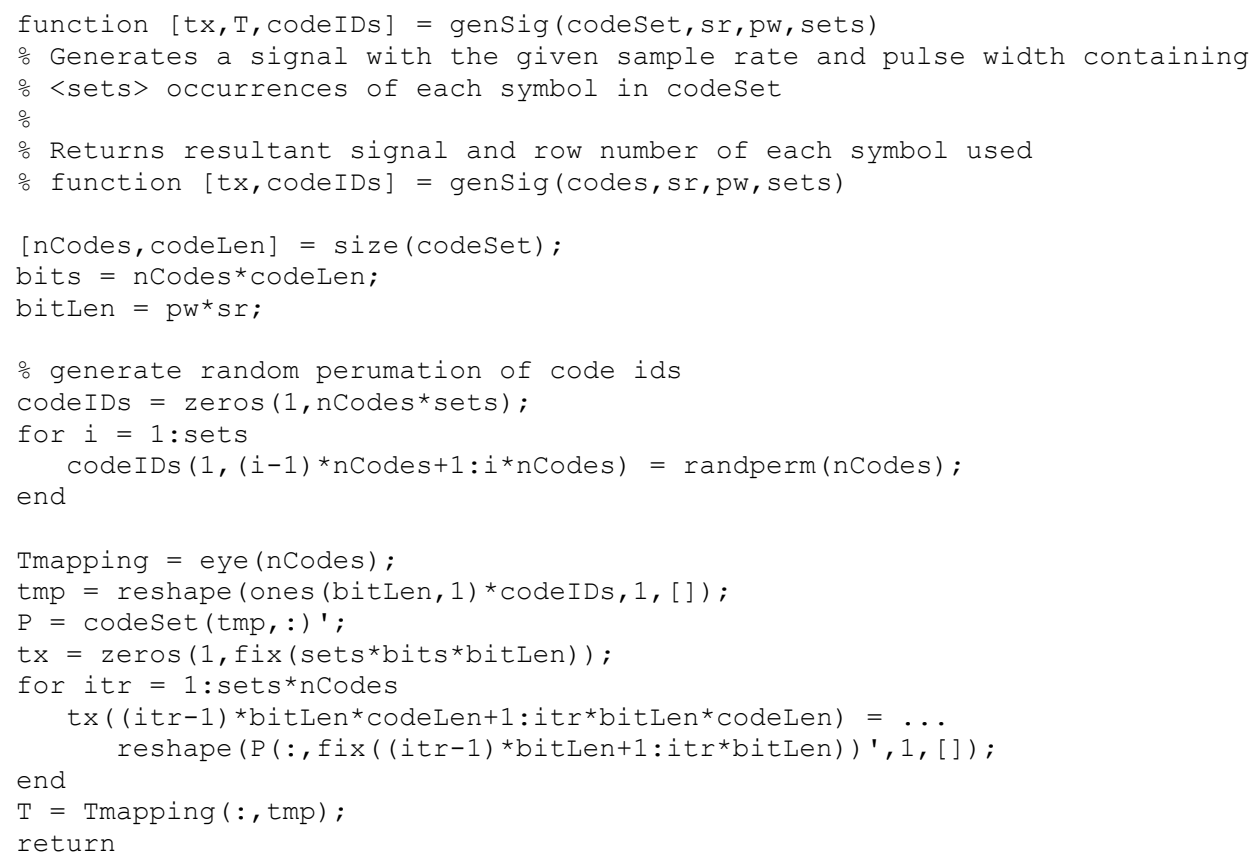

\section{A.3.3 scaleNoiseSNR.m}

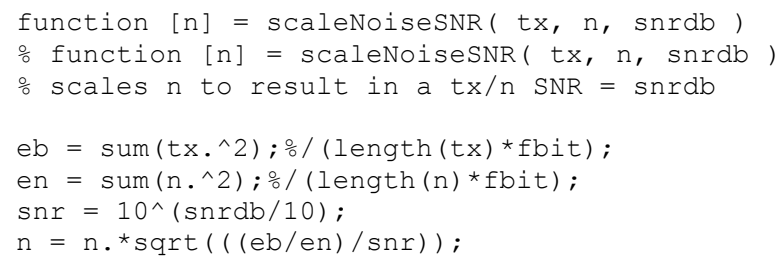

\section{A.3.4 tapSampledSignal.m}

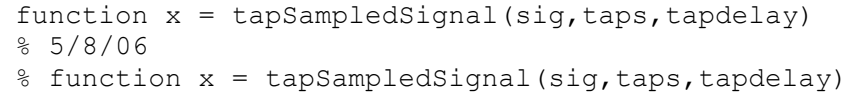




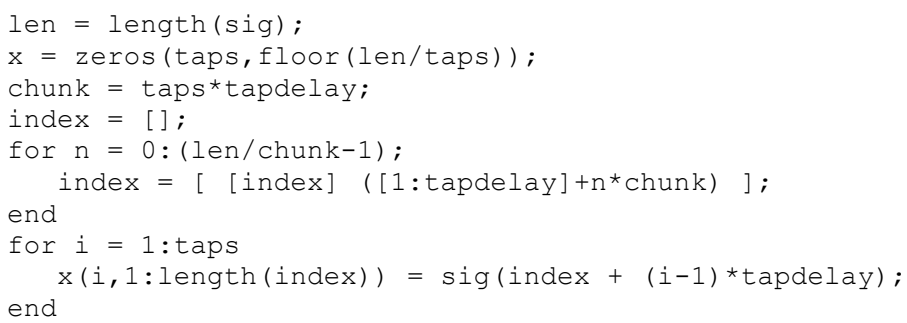

\section{A.4 Training Source Code}

\section{A.4.1 recTrain2.m}

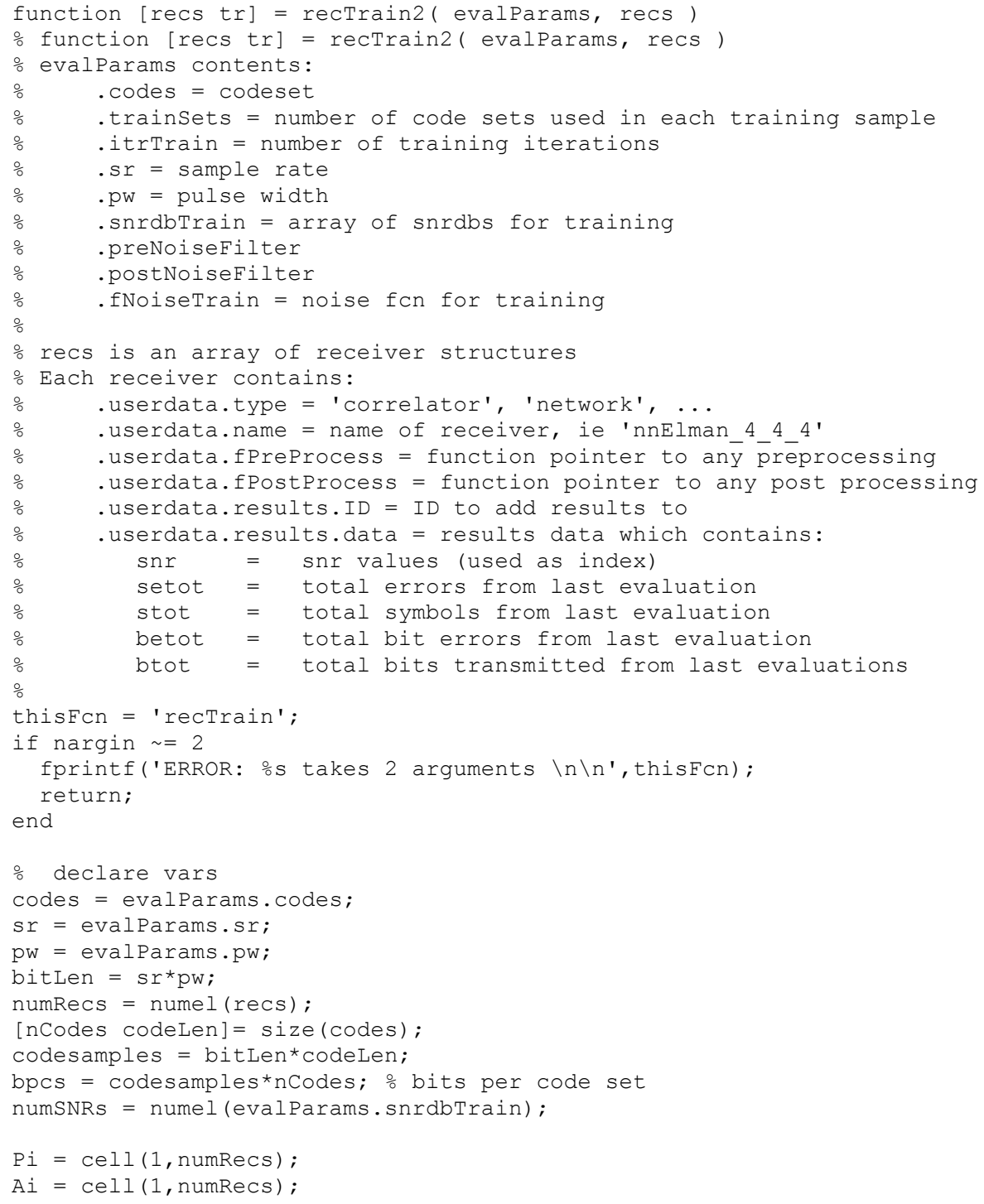




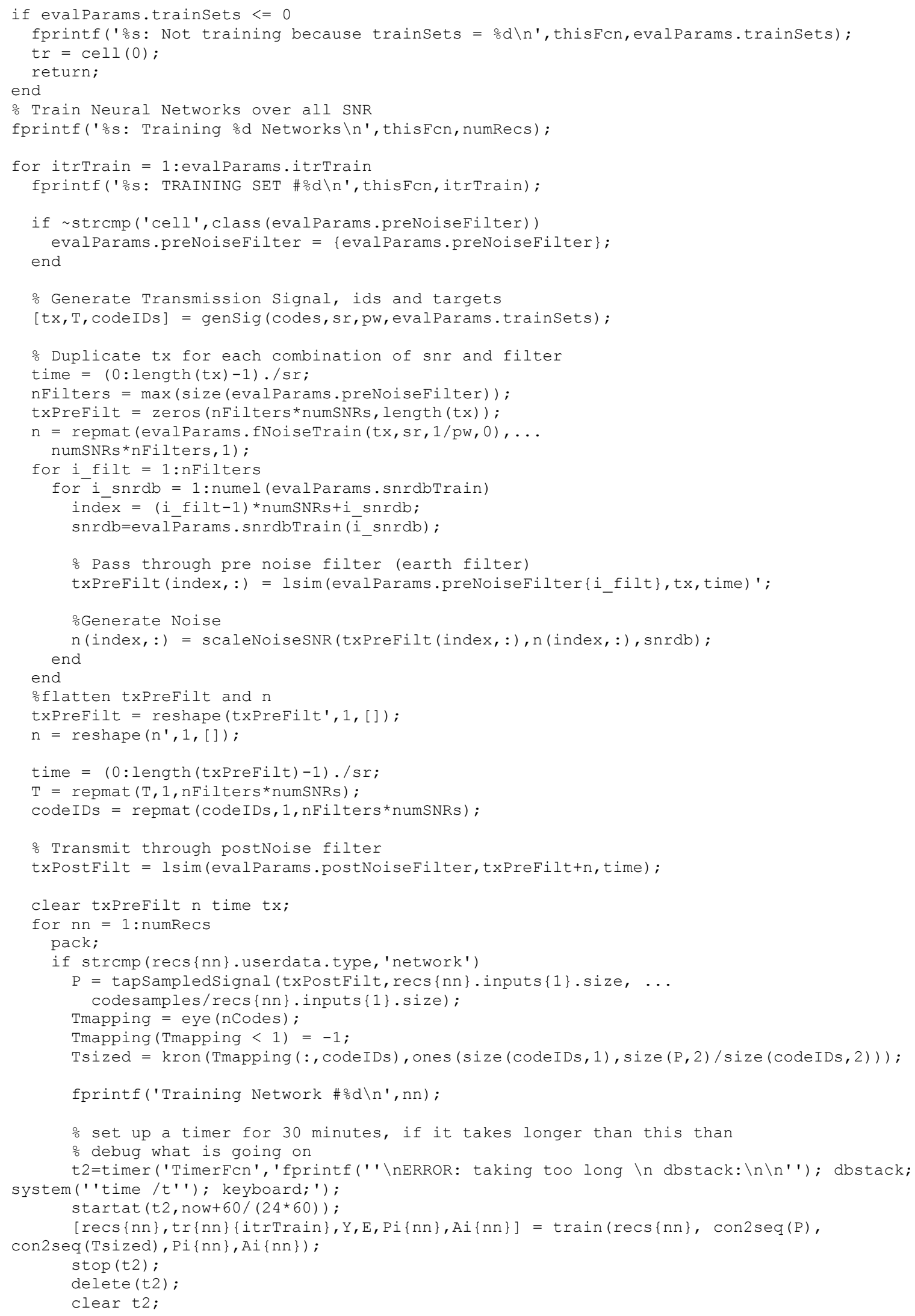




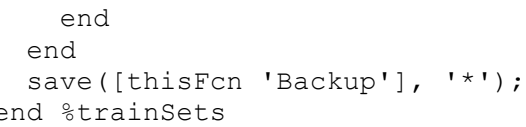

\section{A.5 Test Source Code}

\section{A.5.1 recTest2.m}

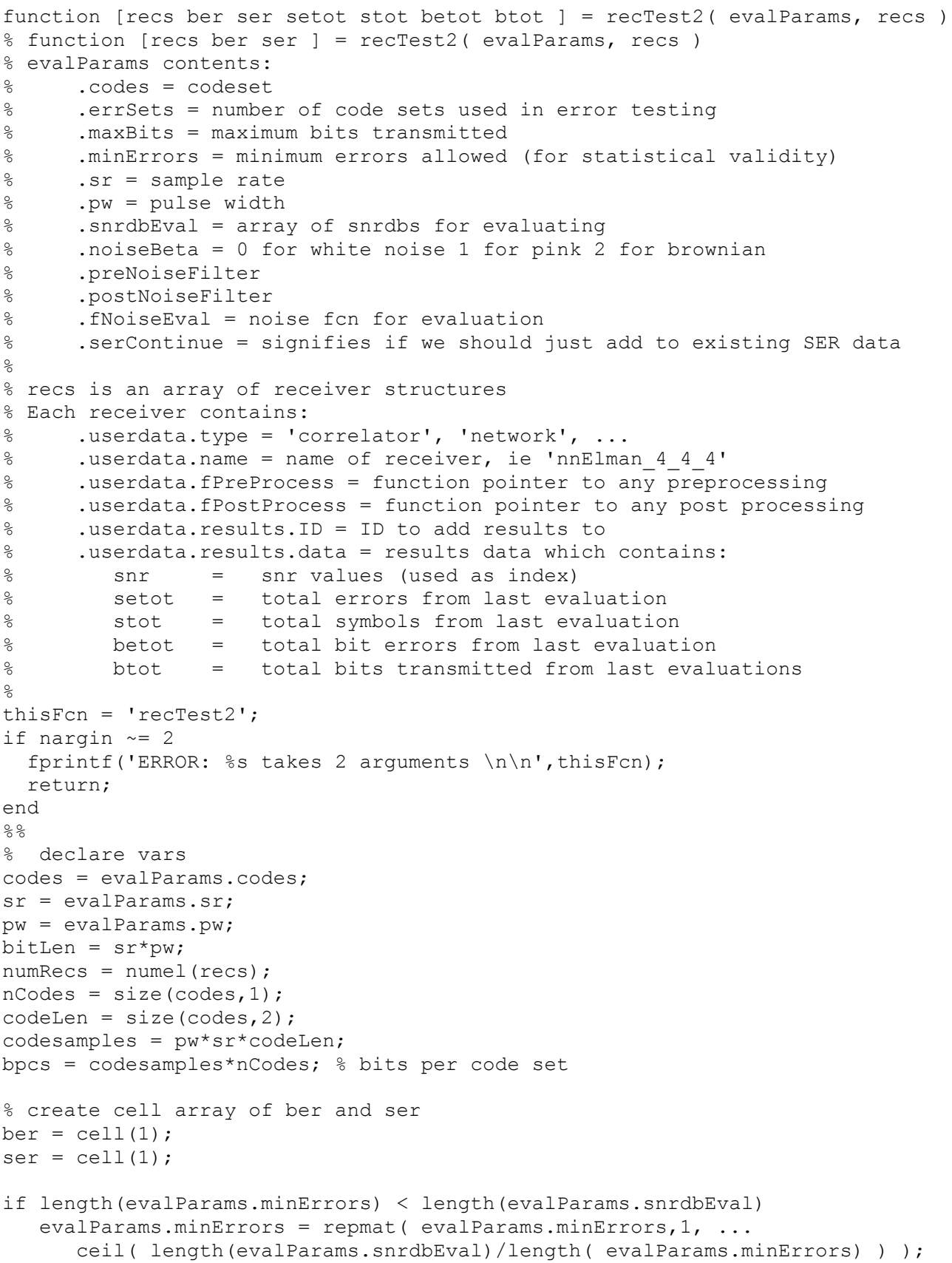




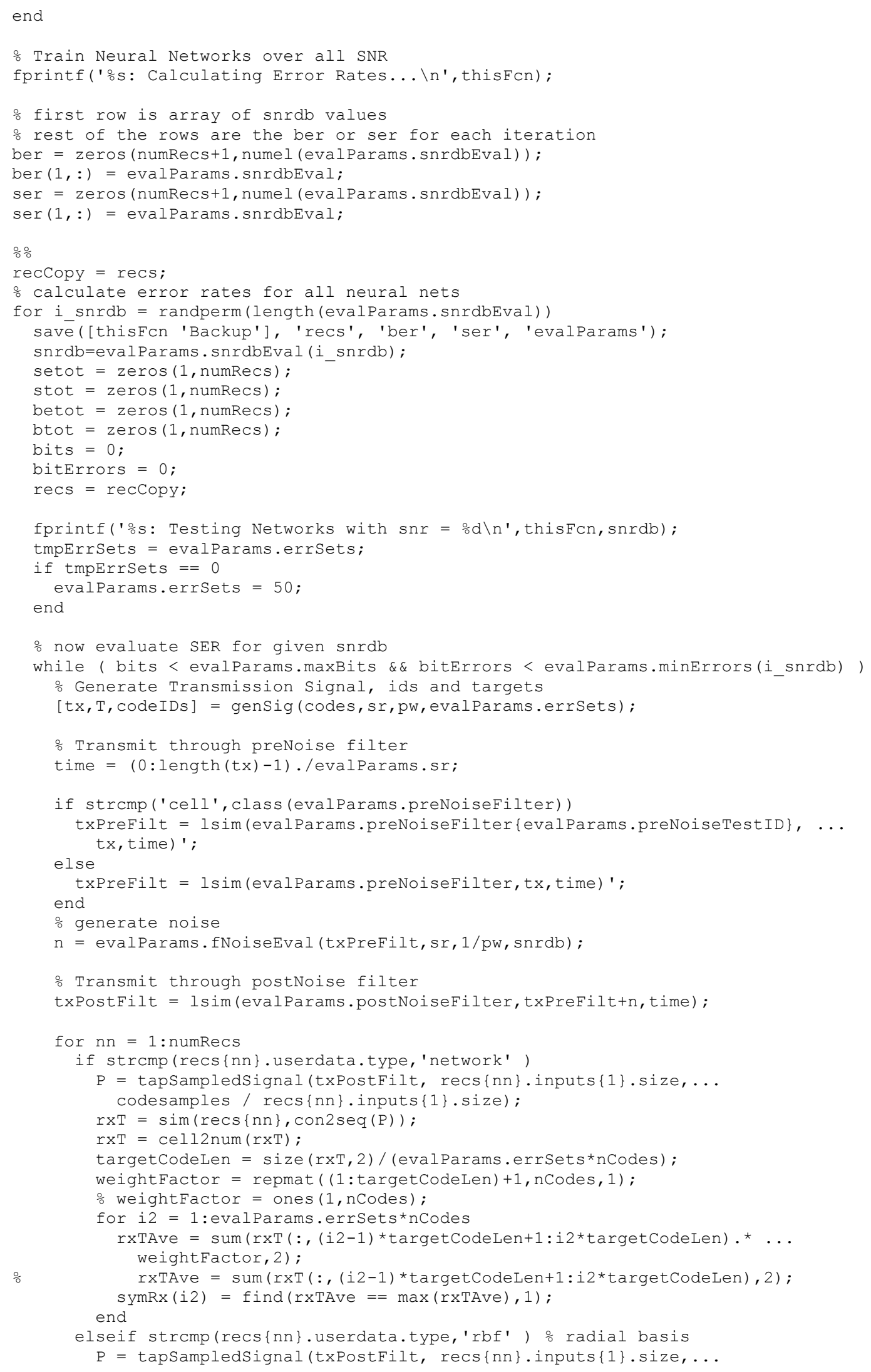




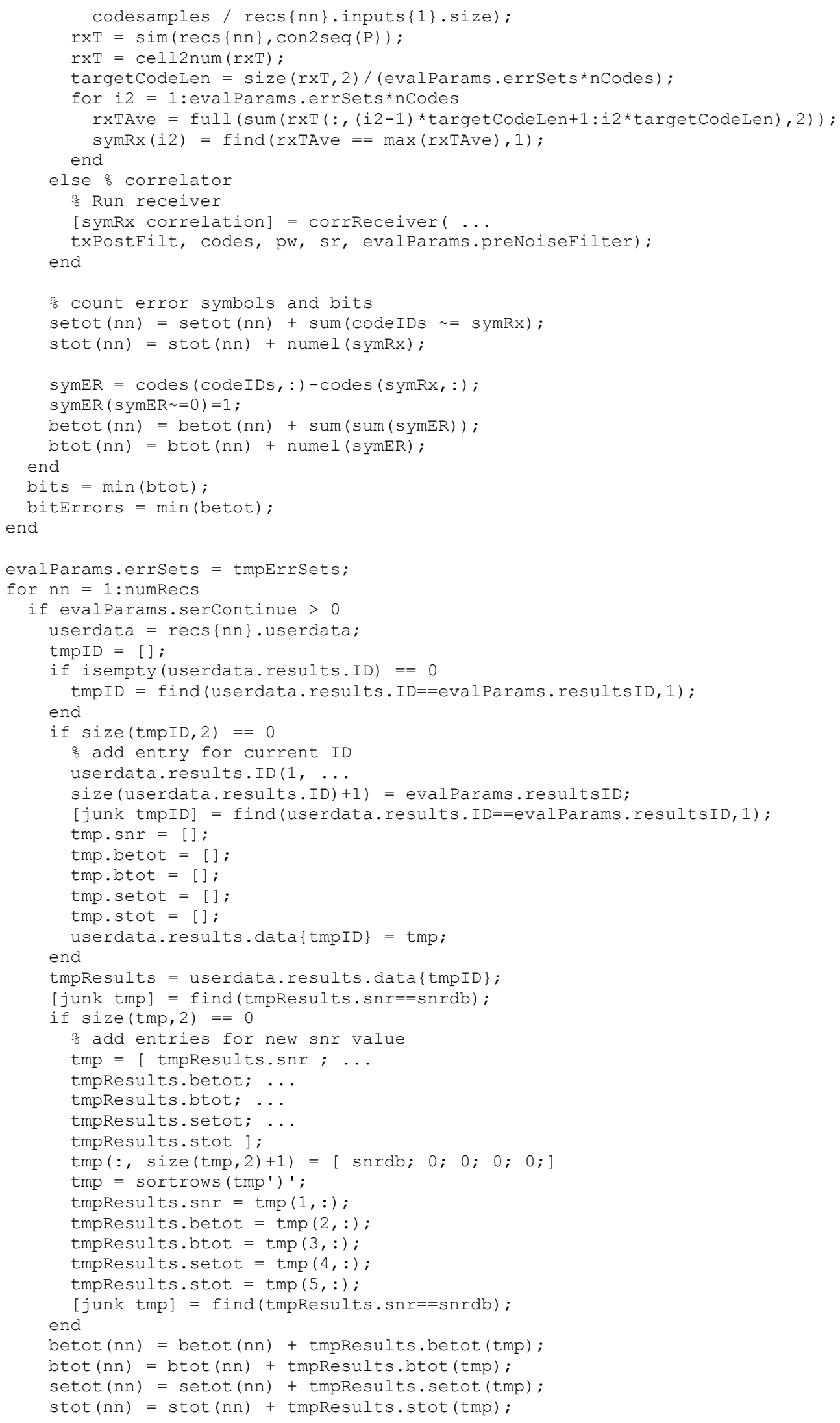




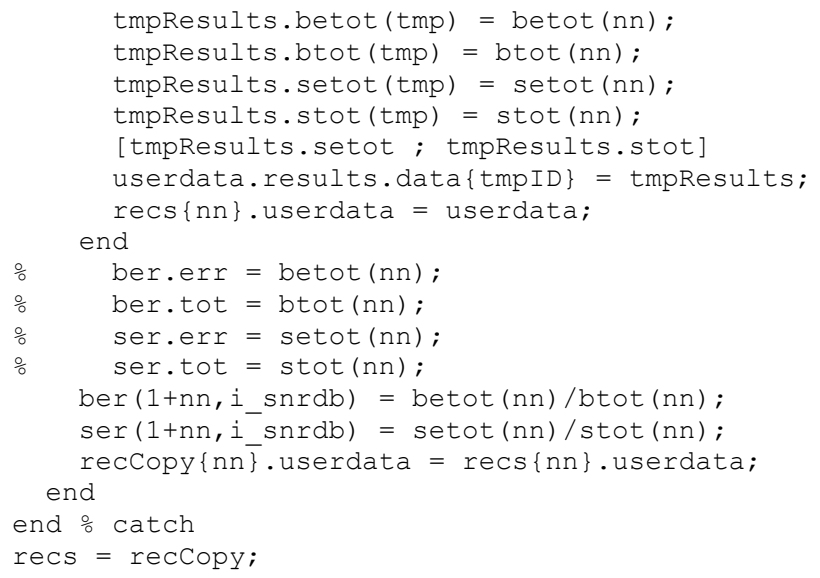

\section{A.6 Example Simulation Scripts}

\section{A.6.1 evalAWGN_4c_25.m}

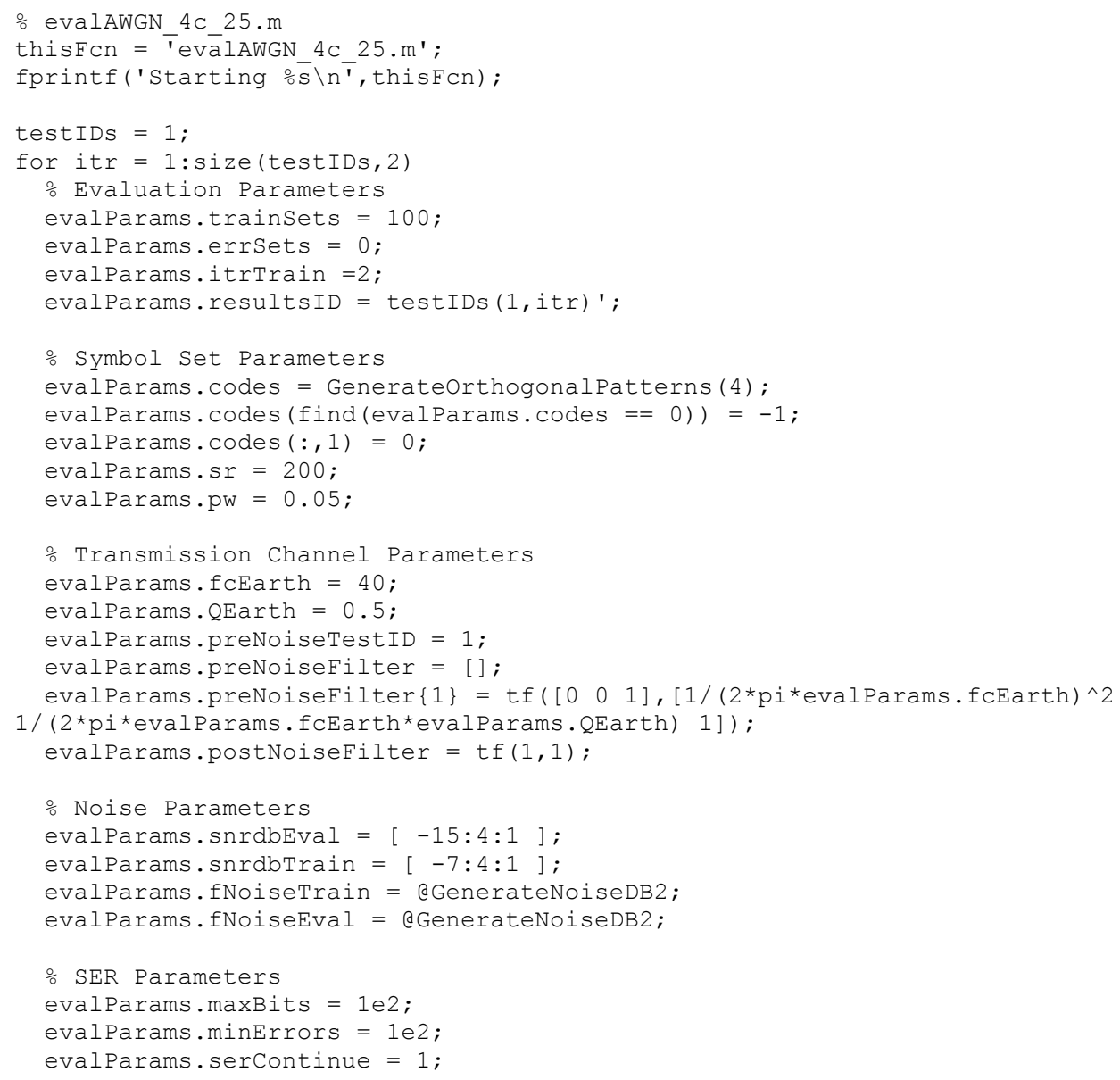




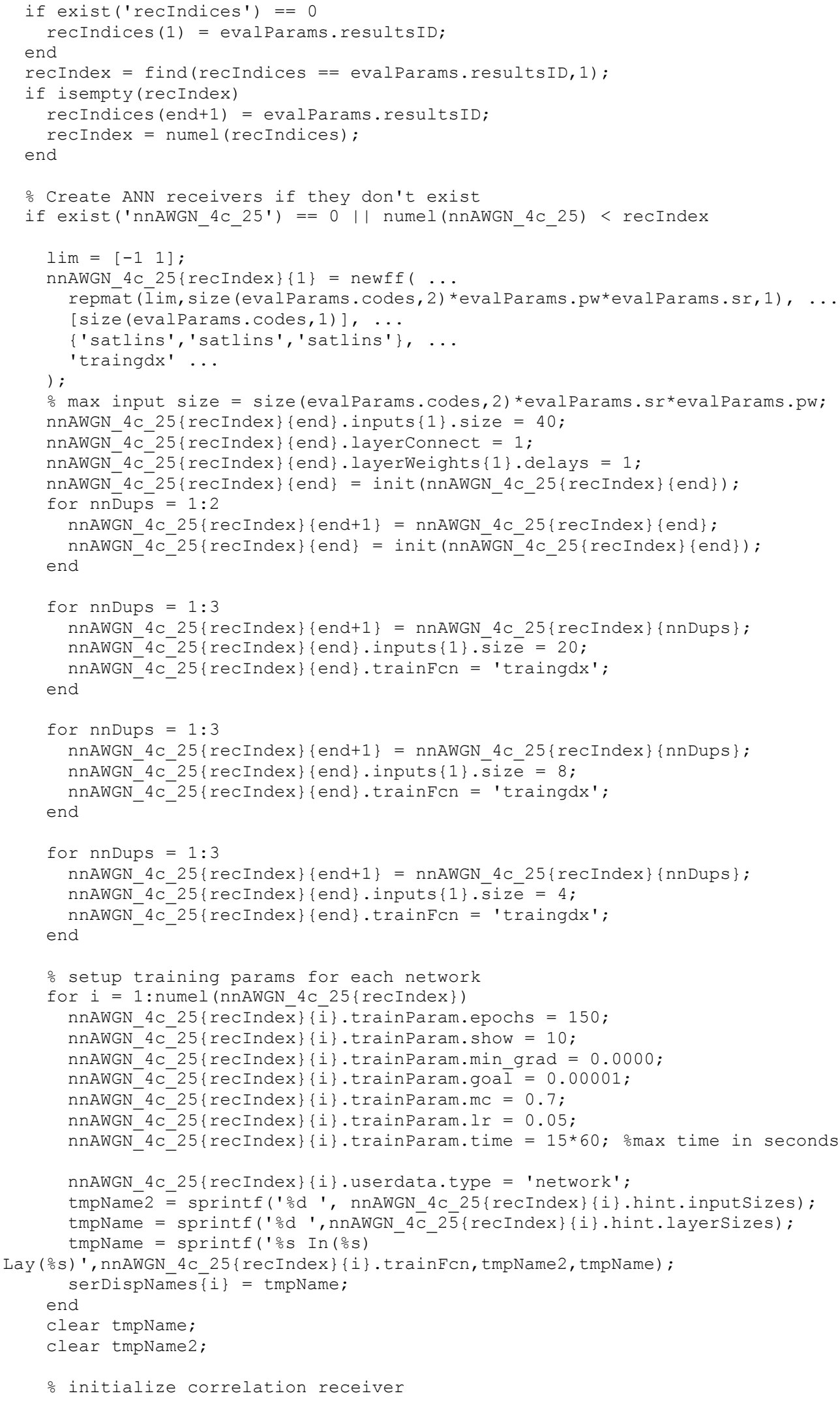




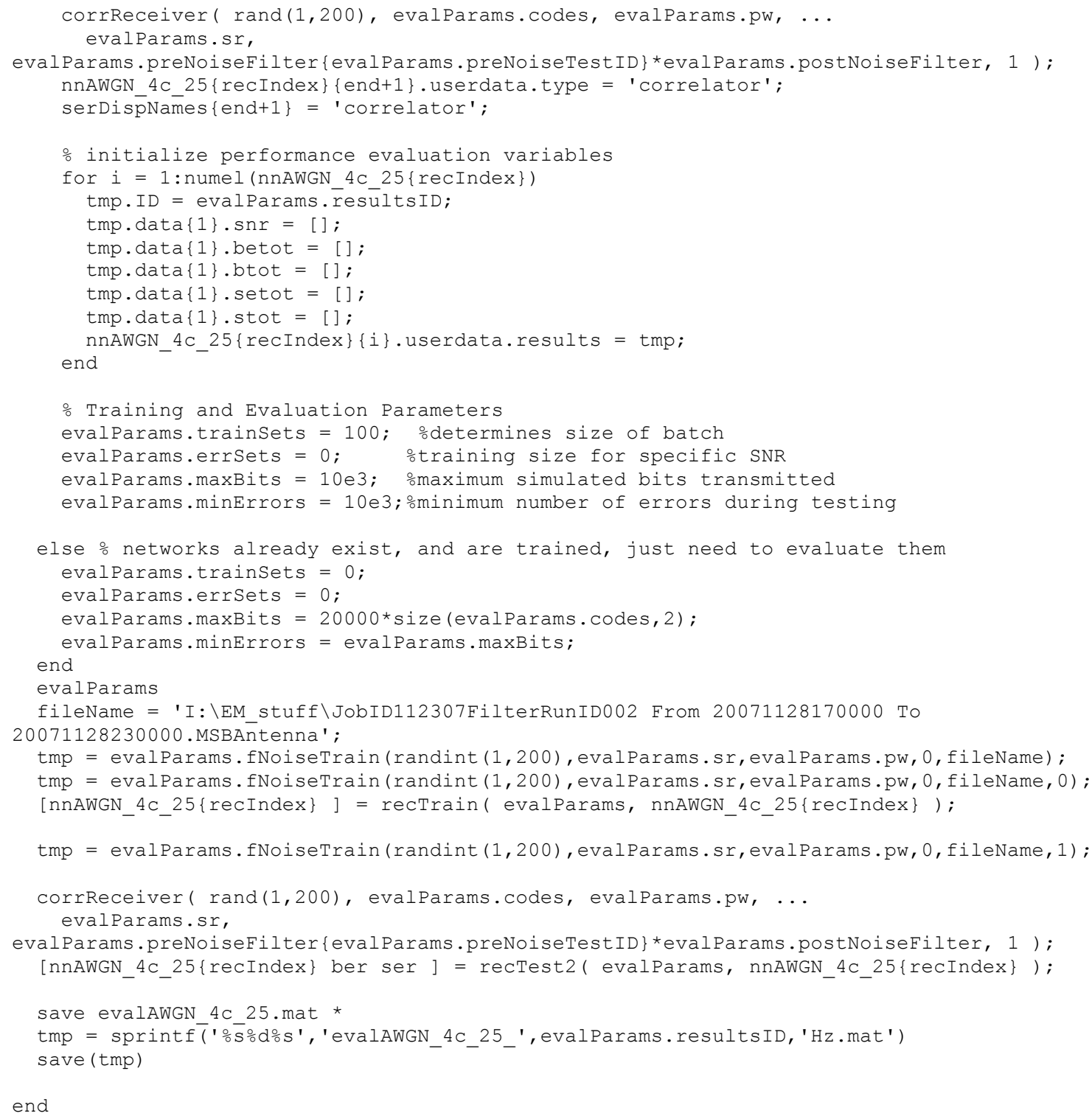

\section{A.6.2 evalAWGN 8c $07 . m$}

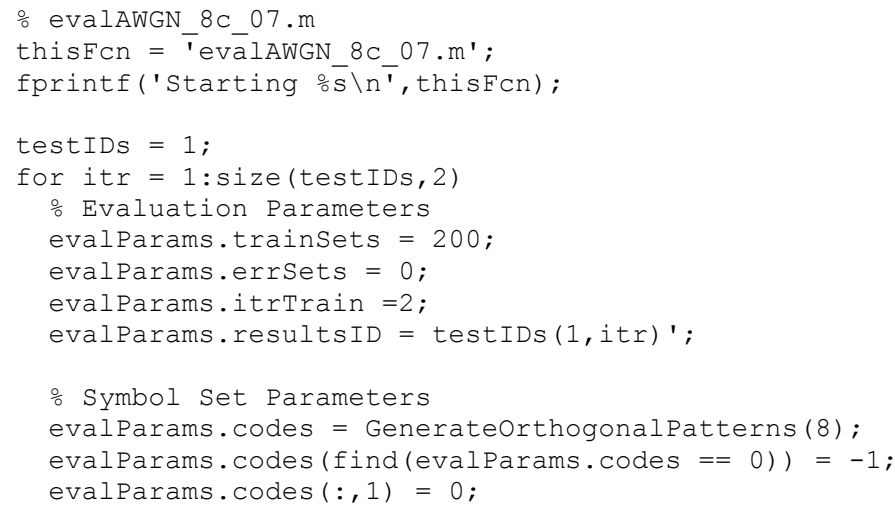




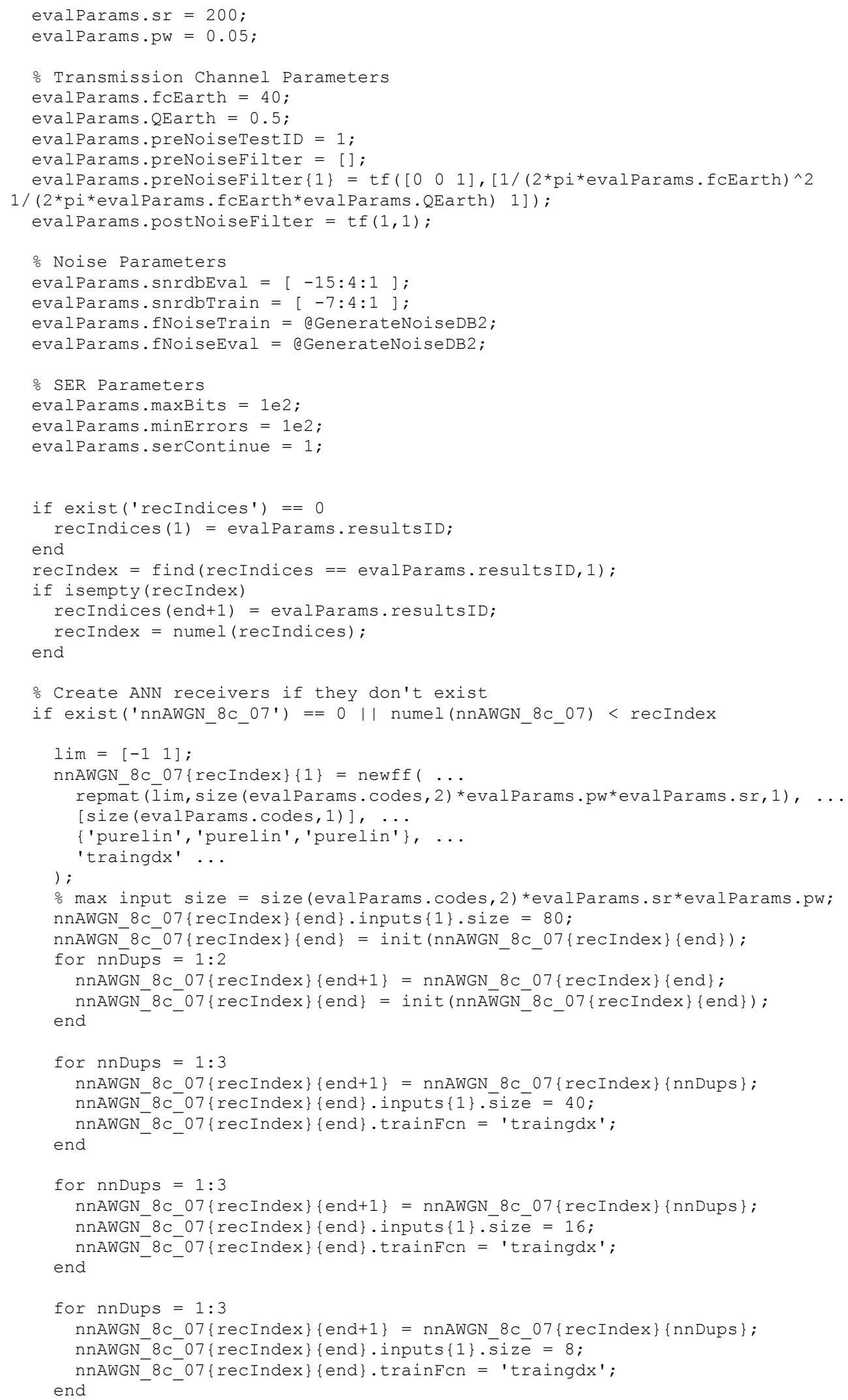




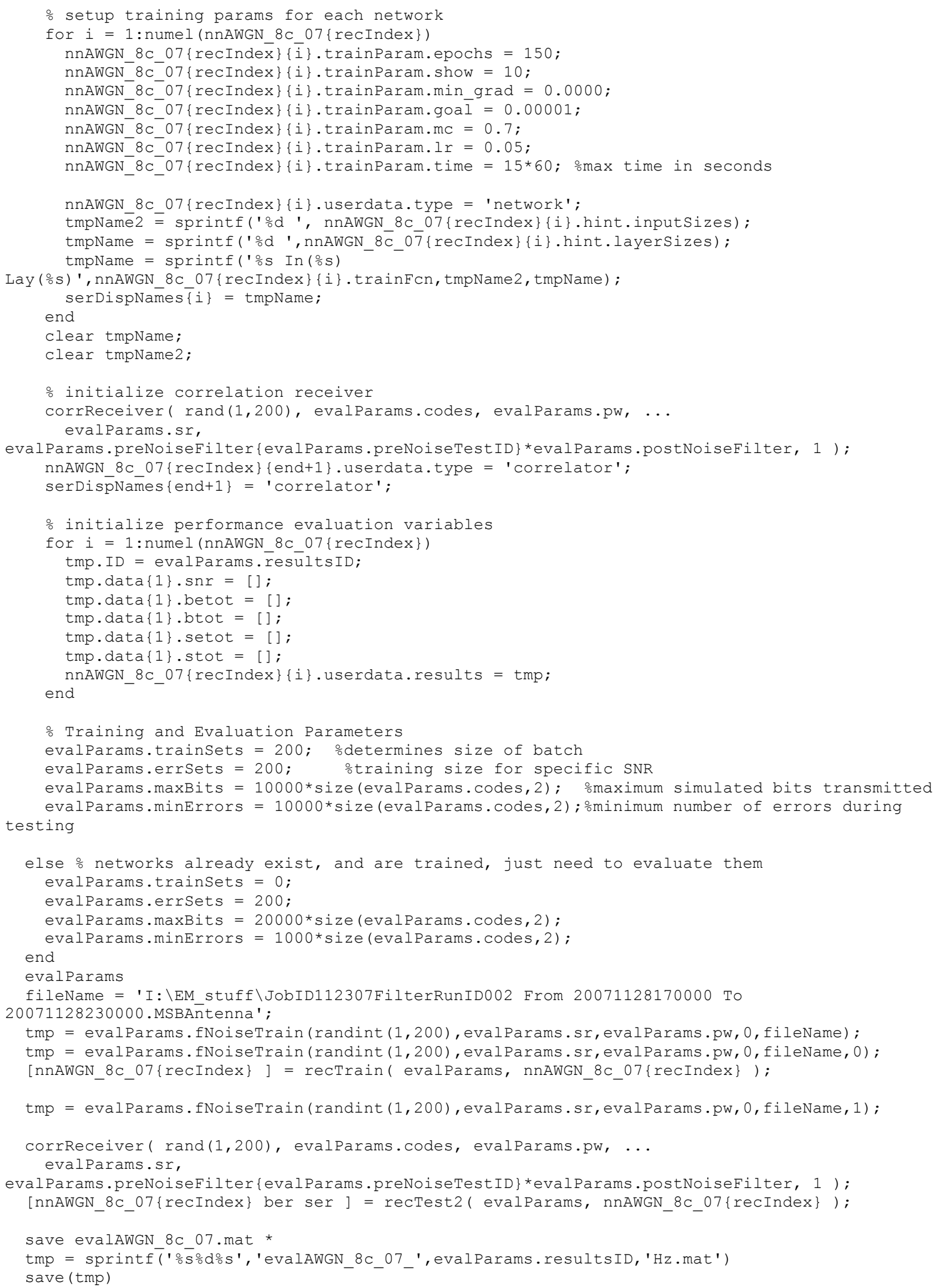




\section{A.6.3 evalShaped_4c_18.m}

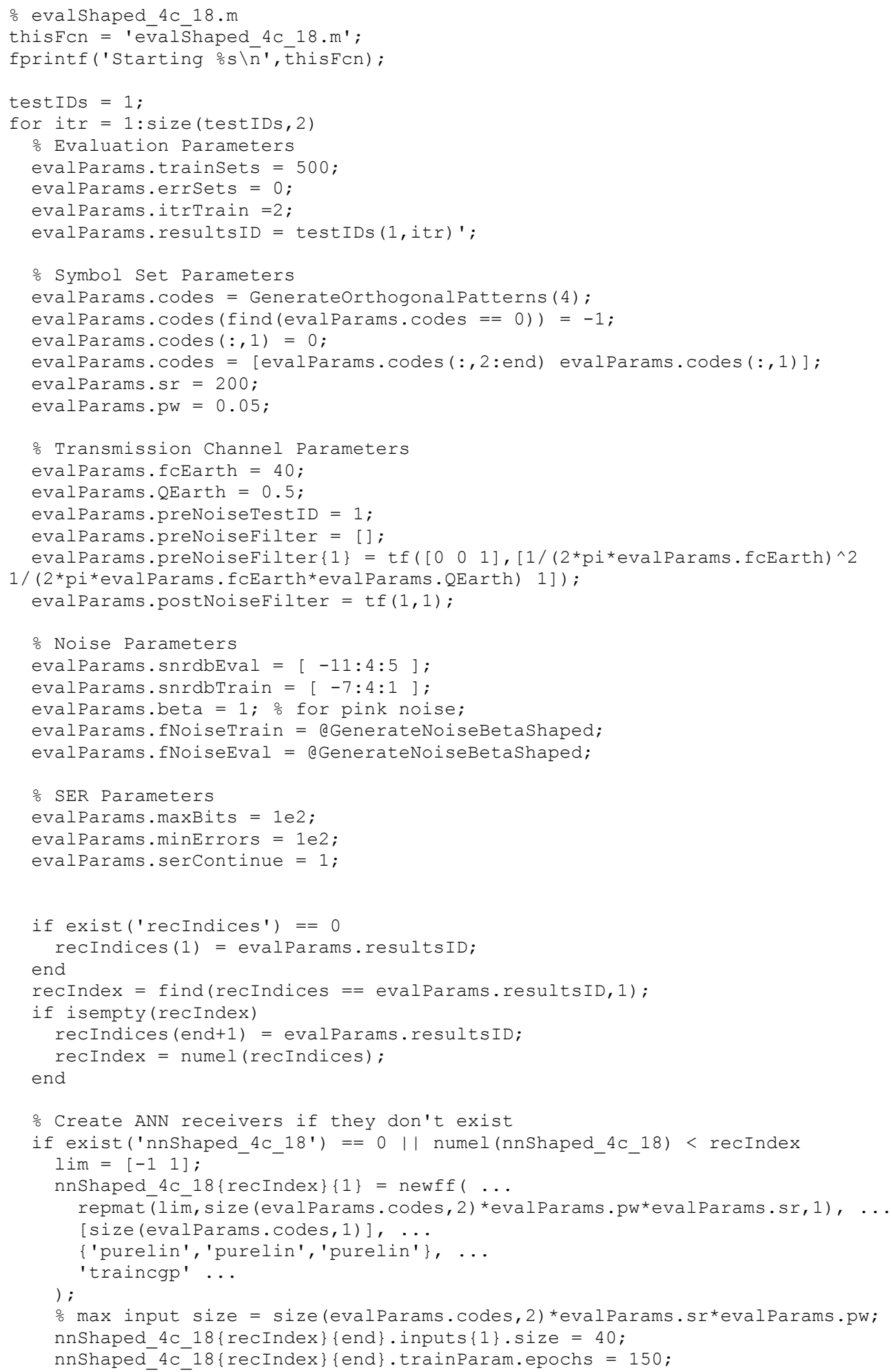




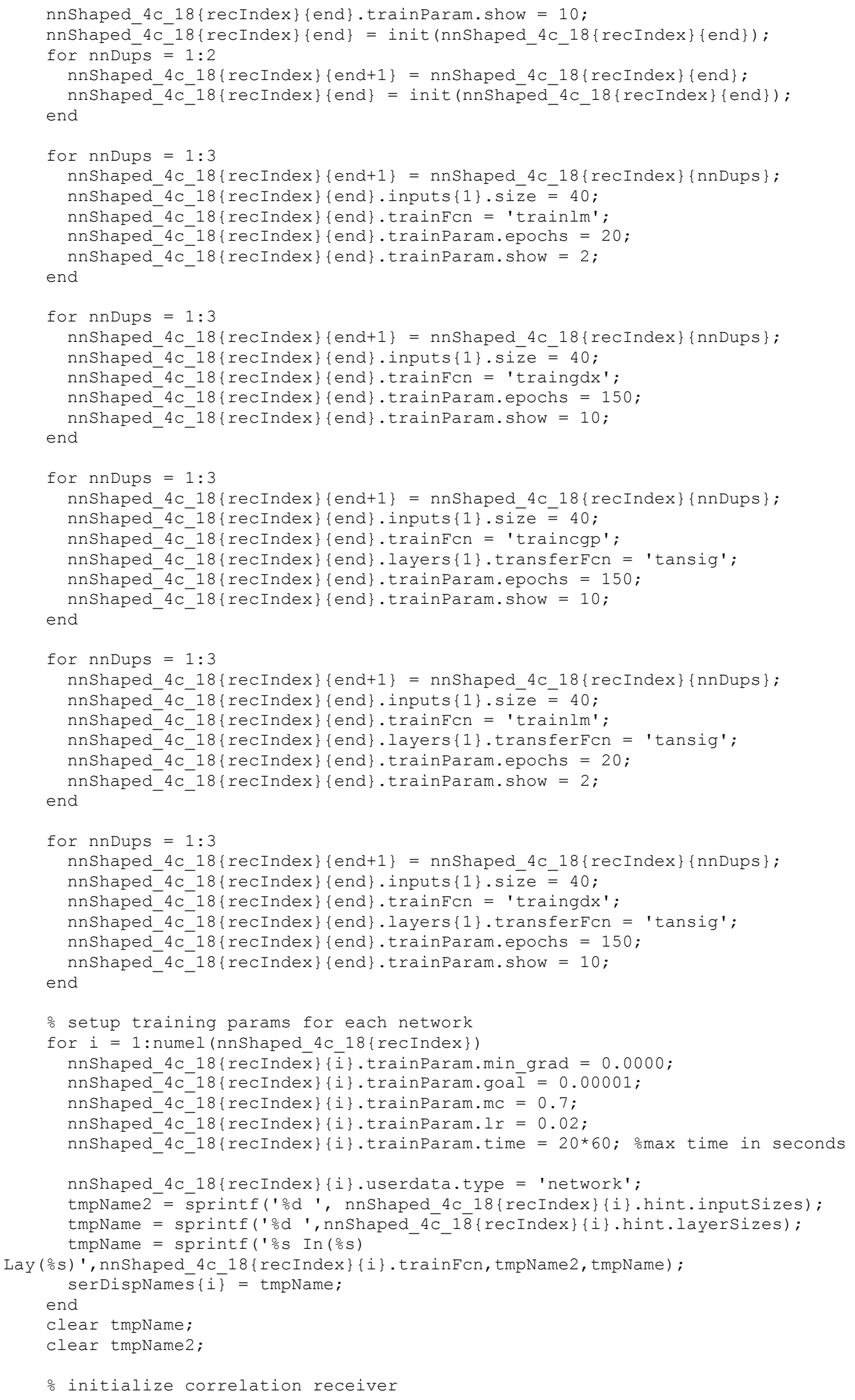




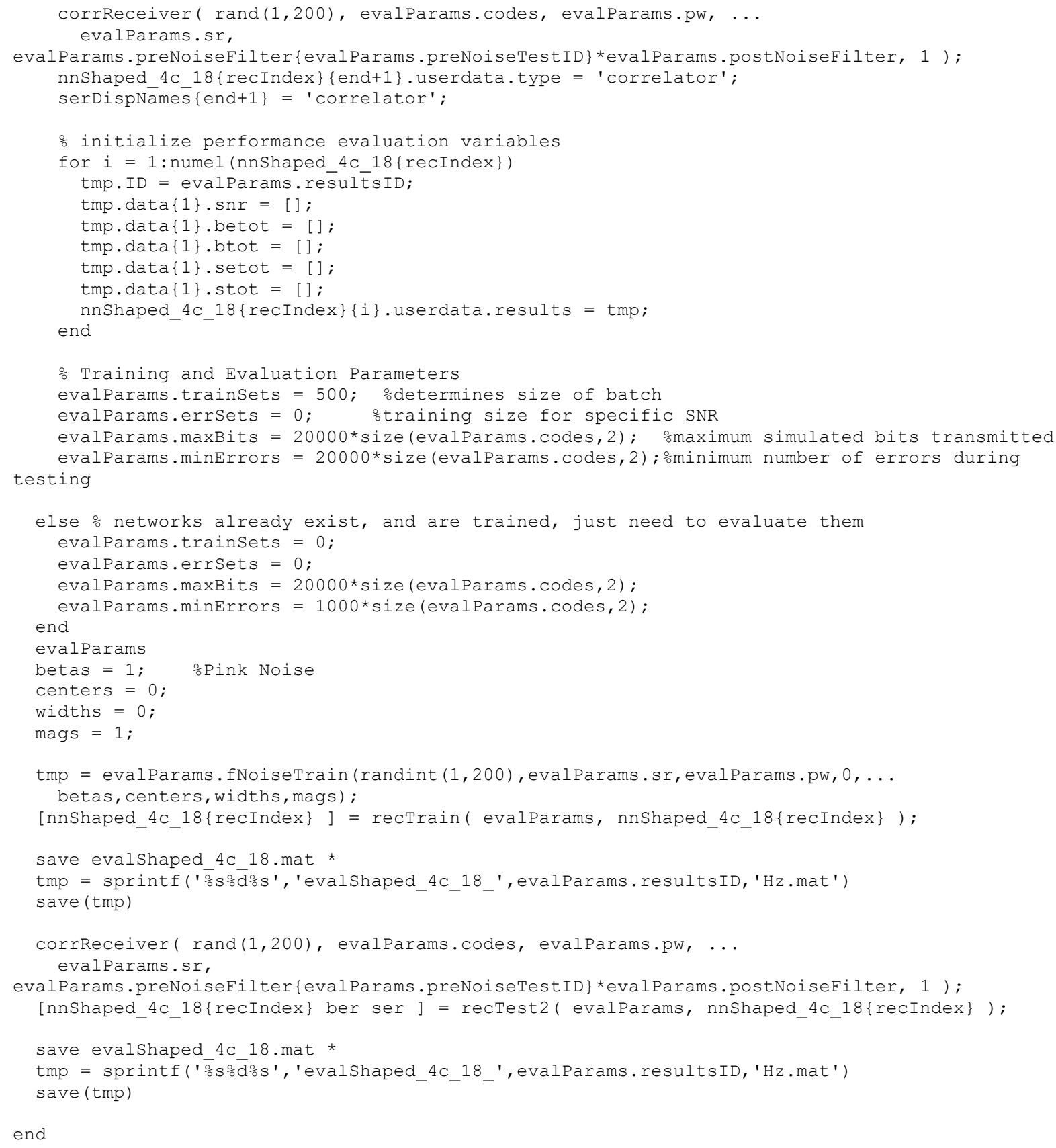

\section{A.6.4 evalField1_4c 01.m}

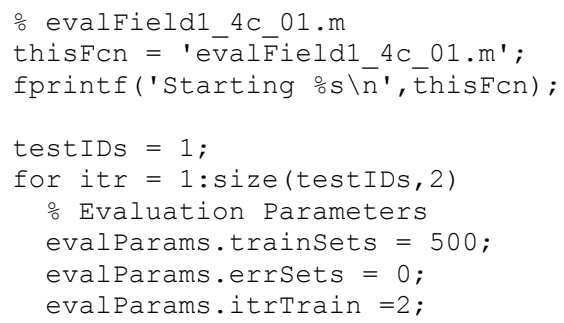




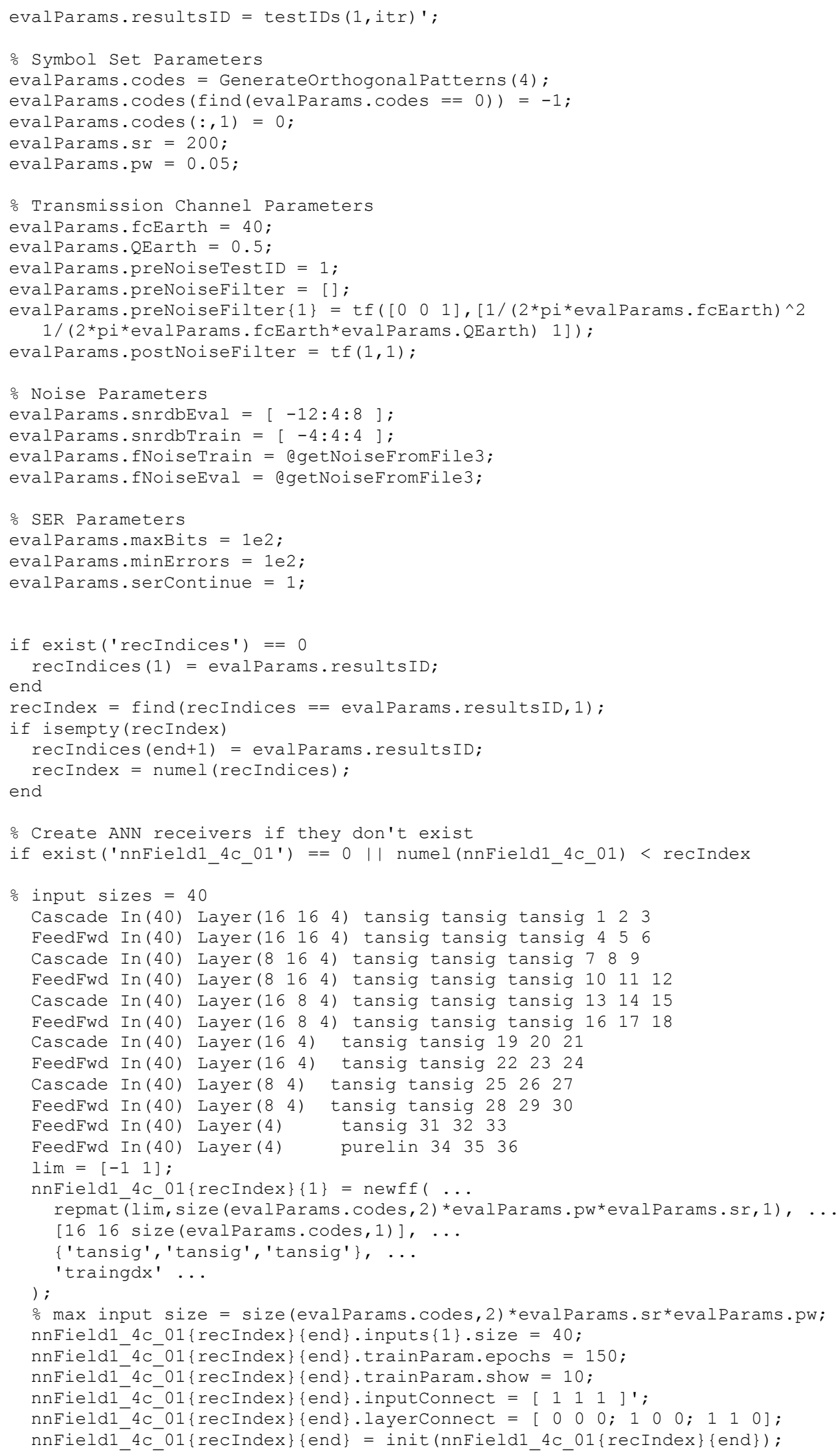




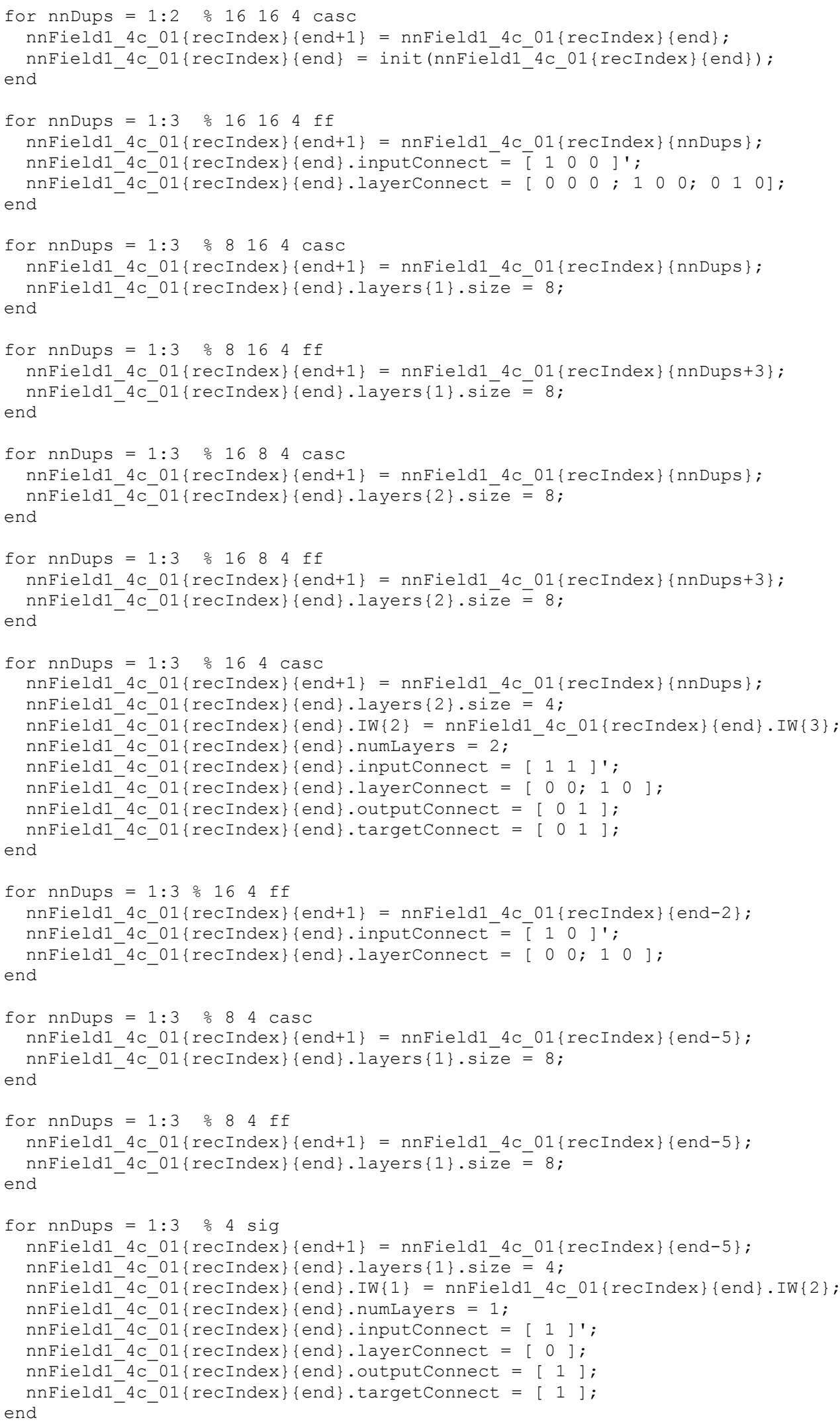




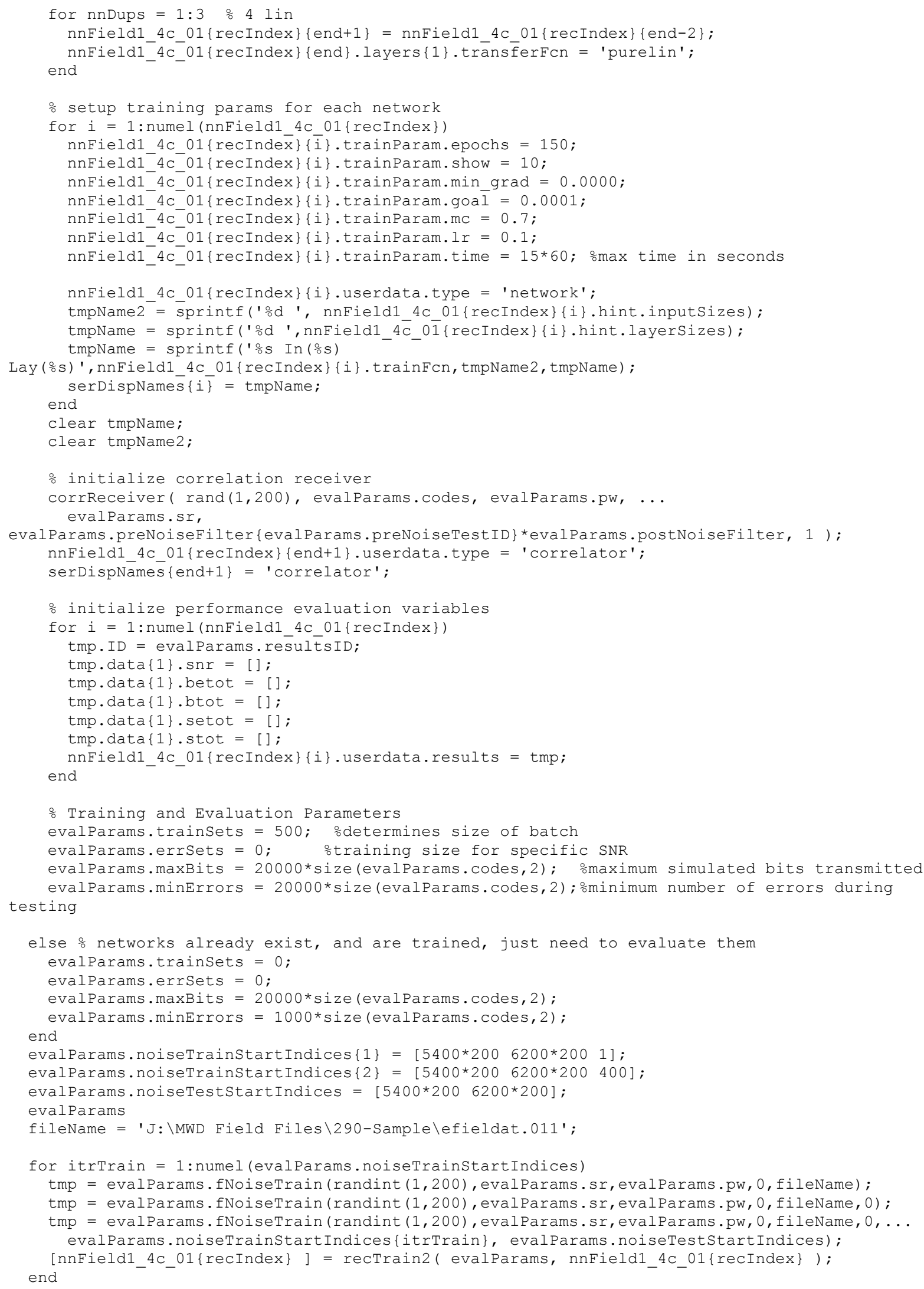




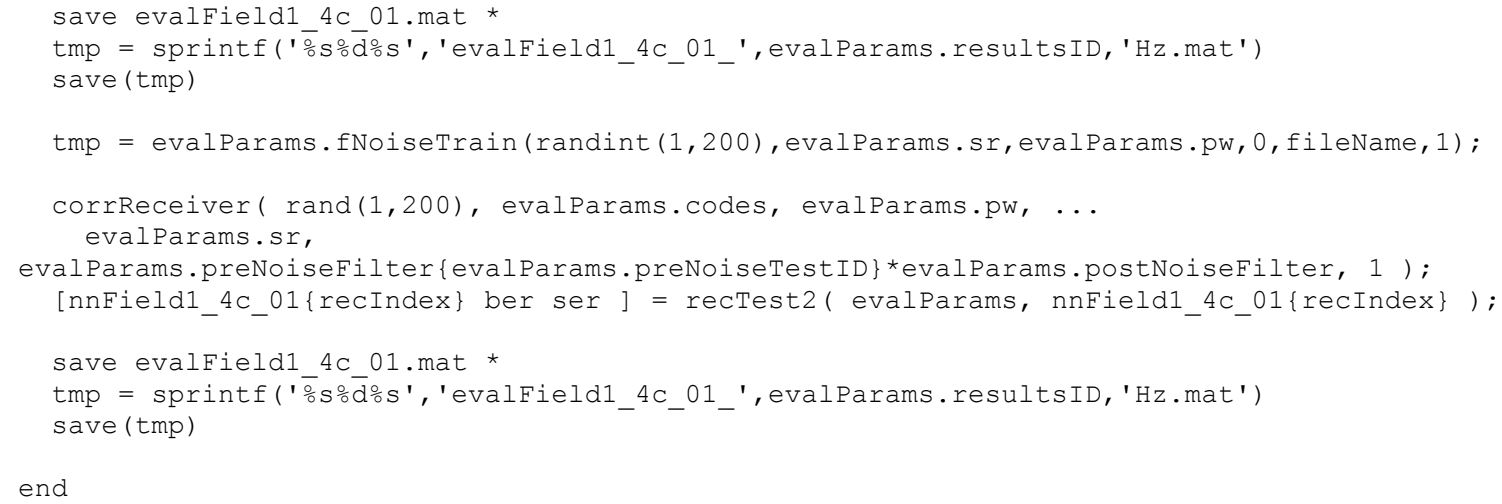

\section{A.7 Data Plotting Source Code}

\section{A.7.1 LoadSerResults2.m}

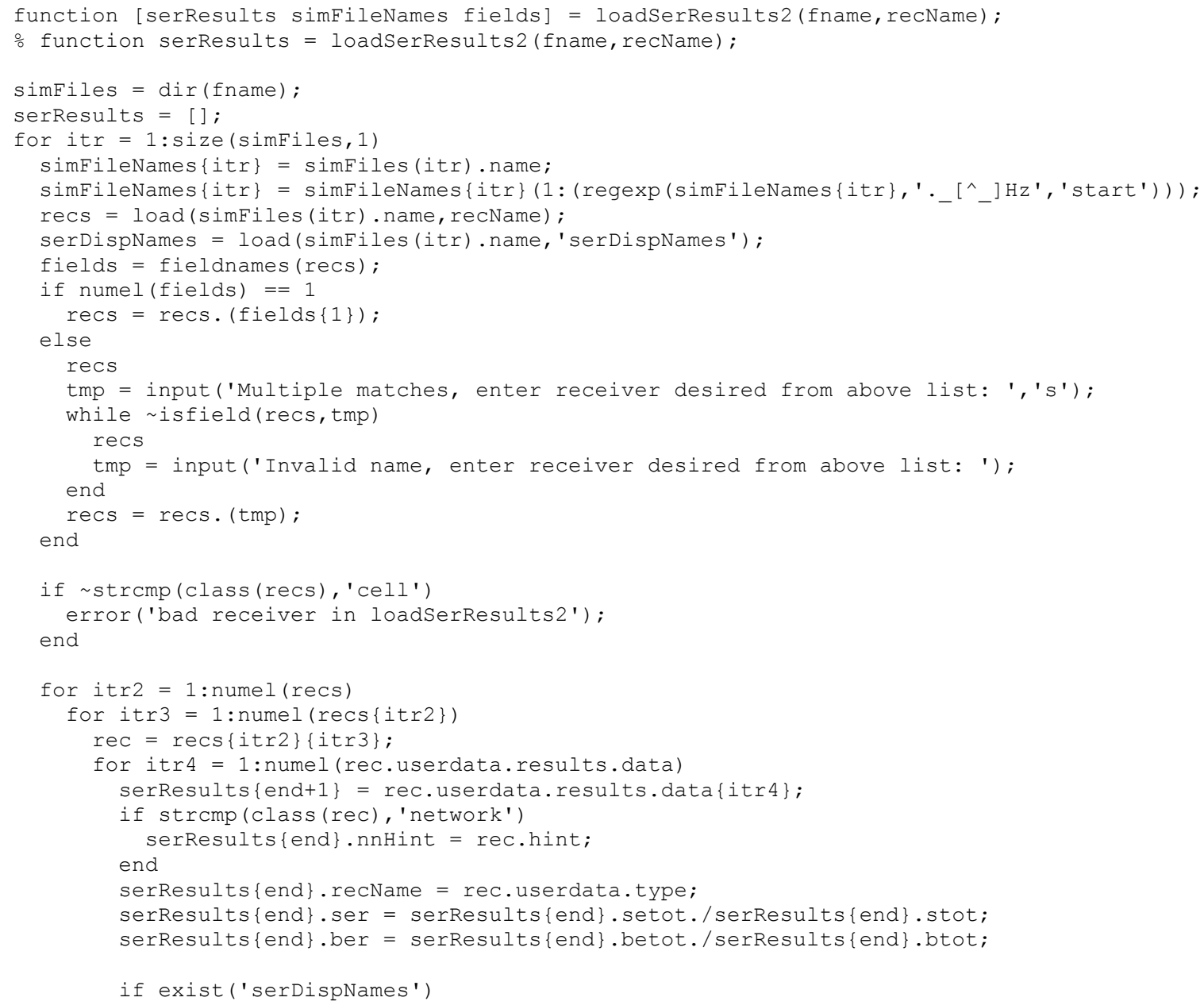




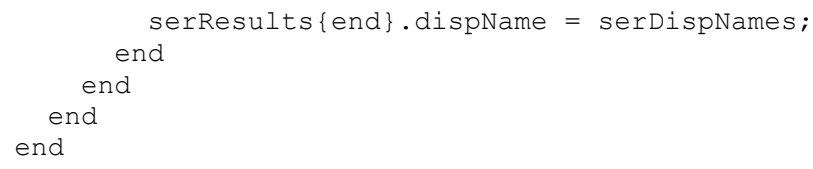

\section{A.7.2 SemilogyBarPlot2.m}

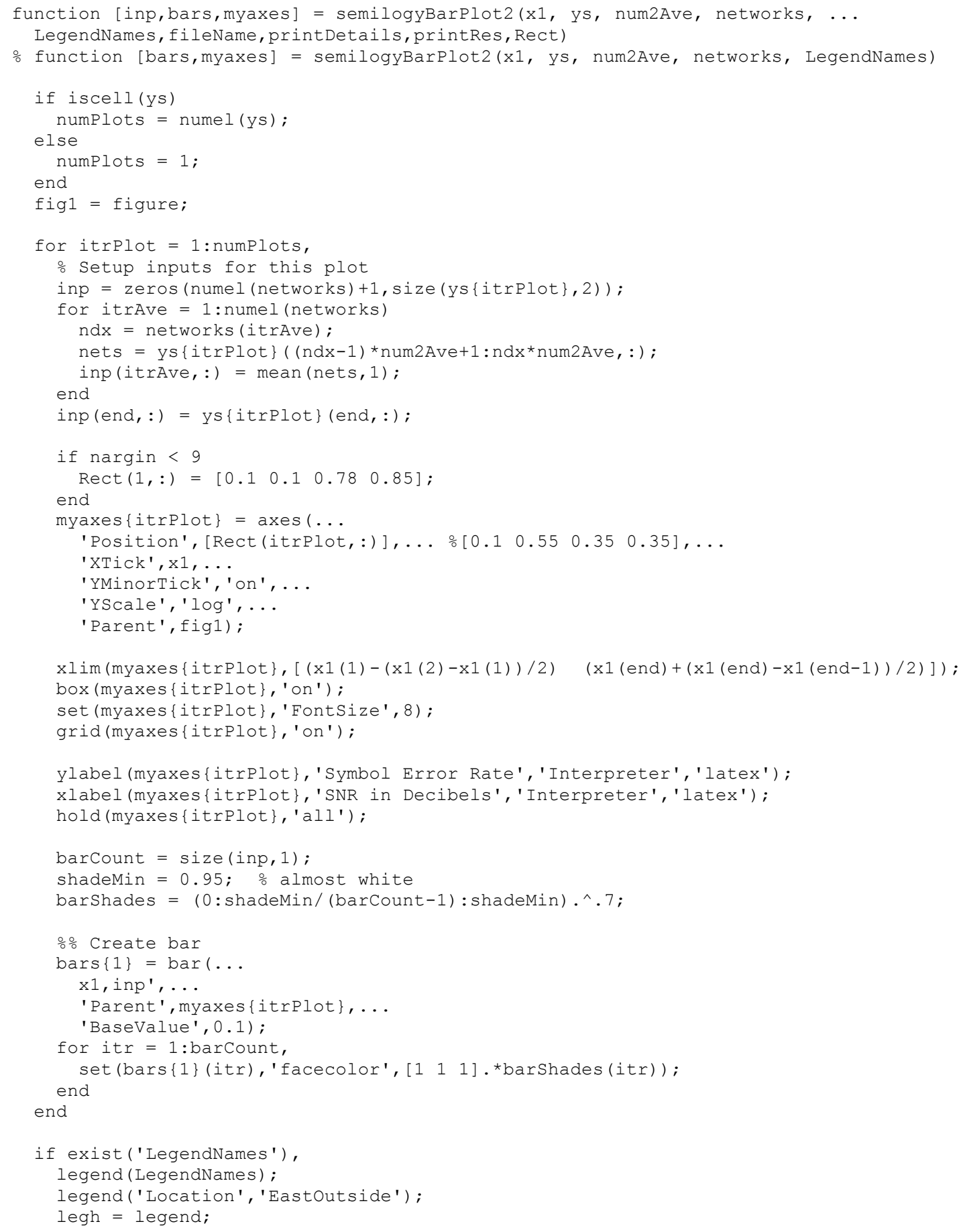




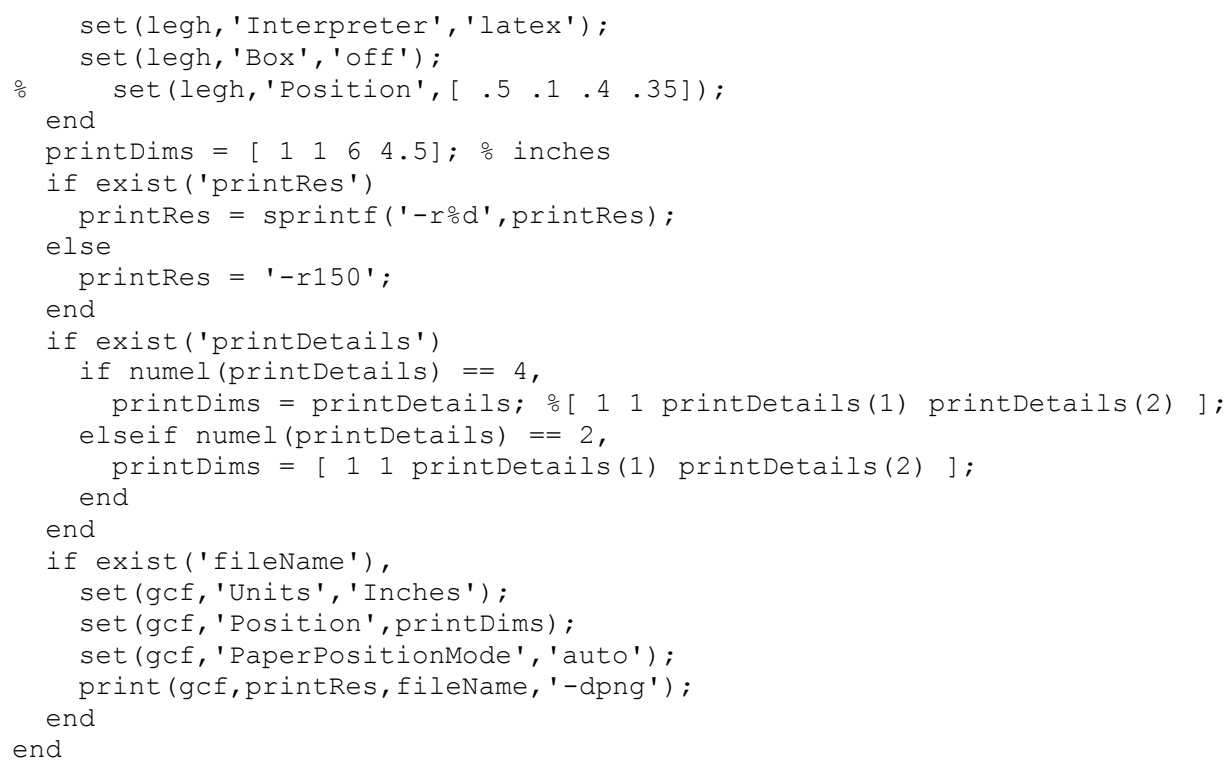

